GWEN EVA JANDA

\title{
Funktionen von Possessivsuffixen in den ugrischen Sprachen
}

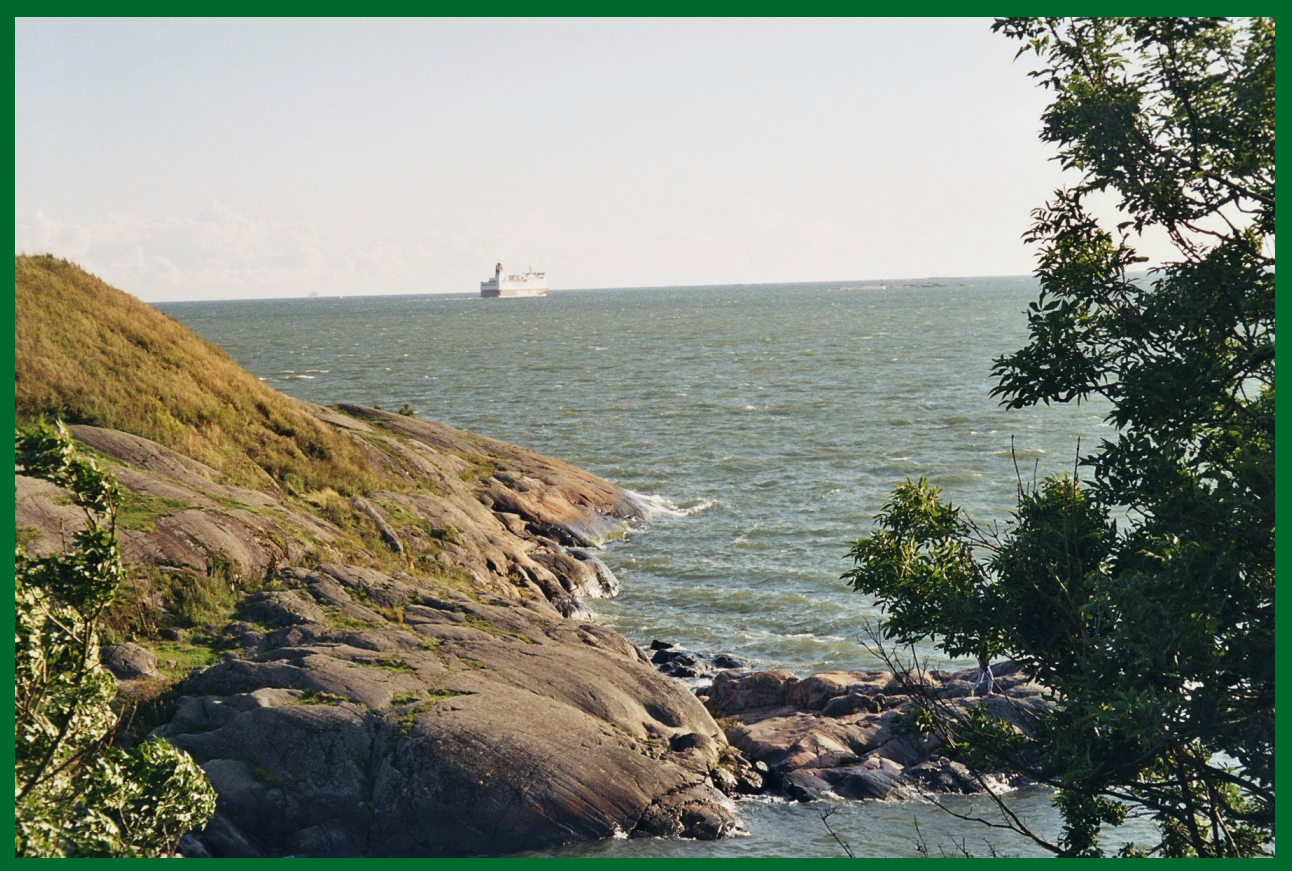


Gwen Eva Janda · Funktionen von Possessivsuffixen in den ugrischen Sprachen 


\section{Herausgegeben von Modern Academic Publishing (MAP) 2019}

MAP (Modern Academic Publishing) ist eine Initiative an der Universität zu Köln, die auf dem Feld des elektronischen Publizierens zum digitalen Wandel in den Geisteswissenschaften beiträgt. MAP ist angesiedelt am Lehrstuhl für die Geschichte der Frühen Neuzeit von Prof. Dr. Gudrun Gersmann.

Die MAP-Partner Universität zu Köln (UzK) und Ludwig-Maximilians-Universität München (LMU) fördern die Open-Access-Publikation von Dissertationen forschungsstarker junger Geisteswissenschaftler beider Universitäten und verbinden dadurch wissenschaftliche Nachwuchsförderung mit dem Transfer in eine neue digitale Publikationskultur.

www.humanities-map.net
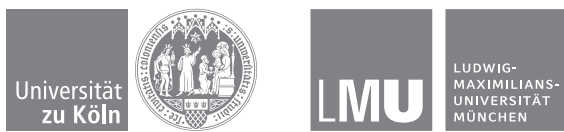
Gwen Eva Janda

\section{Funktionen von \\ Possessivsuffixen in den ugrischen Sprachen}


Herausgegeben von

Modern Academic Publishing

Universität zu Köln

Albertus-Magnus-Platz

50923 Köln

Gefördert von der Ludwig-Maximilians-Universität München

Text @ Gwen Eva Janda 2019

Diese Arbeit ist veröffentlicht unter Creative Commons Licence BY-SA 4.0. Eine Erläuterung zu dieser Lizenz findet sich unter http://creativecommons.org/licenses/ by/4.0/. Diese Lizenz erlaubt die Weitergabe aus der Publikation unter gleichen Bedingungen für privaten oder kommerziellen Gebrauch bei ausreichender Namensnennung des Autors. Grafiken, Tabellen und Abbildungen unterliegen eigenen Lizenzen, die jeweils angegeben und gesondert zu berücksichtigen sind.

Erstveröffentlichung 2019

Zugleich Dissertation der Ludwig-Maximilians-Universität München 2016

Umschlagbild: Schären in der Ostsee, @ C Gwen Eva Janda.

Bibliografische Information der Deutschen Nationalbibliothek

Die Deutsche Nationalbibliothek verzeichnet diese Publikation in der Deutschen Nationalbibliografie; detaillierte bibliografische Daten sind im Internet über http://dnb.dnb.de abrufbar.

ISBN (Hardcover): 978-3-946198-44-4

ISBN (EPUB): 978-3-946198-45-1

ISBN (Kindle): 978-3-946198-46-8

ISBN (PDF): 978-3-946198-47-5

DOI: https://doi.org/10.16994/bal

Herstellung \& technische Infrastruktur:

Ubiquity Press Ltd, 6 Osborn Street, Unit 2N, London E1 6TD, United Kingdom

Open Access-Version dieser Publikation verfügbar unter:

https://doi.org/10.16994/bal

oder Einlesen des folgenden QR-Codes mit einem mobilen Gerät: 


\section{Inhalt}

Danksagung

VII

English Summary

IX

Einleitung

$\mathrm{XI}$

1. Forschungsgegenstand 1

1.1 Die ugrischen Sprachen 1

1.1.1 Typologie der ugrischen Sprachen 1

1.1.2 Die ob-ugrischen Sprachen 2

$\begin{array}{ll}1.1 .3 & 10\end{array}$

1.2 Possessivsuffixe 11

1.2.1 Paradigmen der Possessivsuffixe 11

1.2.2 Konstruktionen mit Possessivsuffixen 18

1.2.2.1 Die attributive Possessivkonstruktion 19

1.2.2.2 Weitere Konstruktionstypen 22

2. Forschungsstand 27

$2.1 \quad$ Von der Funktion zur Form - die onomasiologisch-basierte Forschung 28

2.2 Spezifische Abhandlungen zur Possessivkonstruktion 32

2.3 Spezifische Abhandlungen zu Possessivmarkern 34

2.3.1 Exkurs: Areallinguistik 41

2.3.2 Possessivpronomina 42

2.3.3 Die Definitheits-Theorie 43

2.3.4 Neuere Forschungsansätze nach der Definitheit 49

2.3.5 Die Forschungsansätze zum Possessivsuffix in anderen uralischen Sprachen $\quad 60$

3. Theoretische Grundlagen 63

3.1 Kognitive Linguistik 63

3.2 Funktionale Grammatik 66

$\begin{array}{lll}\text { 3.2.1 Textlinguistik } & 70\end{array}$

3.2.2 Referenzlinguistik 72

3.3 Die Referentialität des Possessivsuffixes 80

3.4 Exkurs: Evaluation des Ansatzes zur Definitheit 82

4. Daten und Methodik 89

4.1 Daten und Quellen 89

4.1.1 Ob-Ugrisch 89

4.1.2 Ungarisch 91

4.2 Methodik 93

4.2.1 Glossierung, Adaption 93 
4.2.2 Auswahl und Systematisierung der Daten 96

4.2.3 Analyse der einzelnen Belege 100

5. Ergebnis und Diskussion 105

$\begin{array}{lll}5.1 & \text { Die einzelnen Konstruktionen } & 105\end{array}$

5.1.1 Die Konstruktion Substantiv-Possessivsuffix (SuBs-Px) 105

$\begin{array}{lll}\text { 5.1.1.1 Attribute der Konstruktion SuBs-Px } & 105\end{array}$

$\begin{array}{lll}\text { 5.1.1.2 } & \text { Realisierung des Modifizierers } & 107\end{array}$

5.1.1.3 In der Konstruktion auftretende Determinantien 113

5.1.1.4 Lesart der Relation zwischen Modifizierer und Kopf 115

5.1.2 Die Konstruktion Verbalnomen-Possessivsuffix (VN-Px) 123

$\begin{array}{ll}\text { 5.1.2.1 Infinitiv } & 124\end{array}$

$\begin{array}{lll}\text { 5.1.2.2 Partizip } & 125\end{array}$

$\begin{array}{lll}\text { 5.1.2.3 Konverb } & 130\end{array}$

5.1.3 Die Konstruktion Postposition-Possessivsuffix (PostP-Px) 130

5.1.3.1 Die Konstruktion PostP-Px im Ungarischen 133

5.1.3.2 Die Konstruktion PostP-Px im Ob-Ugrischen 134

$\begin{array}{lll}5.2 & \text { Die Konstruktionen und die Textumgebung } & 135\end{array}$

$\begin{array}{ll}\text { 5.2.1 Pragmatisch motivierte Mechanismen } & 137\end{array}$

$\begin{array}{lll}\text { 5.2.1.1 Anchoring } & 137\end{array}$

5.2.1.2 Re-Aktivierung 140

5.2.1.3 Gemeinsame Vorerwähnung von Referenzpunkt und Target 147

5.2.1.4 Die Textumgebung der Konstruktion VN-Px 150

5.2.2 Syntaktisch motivierte Mechanismen 152

$\begin{array}{ll}\text { 5.2.2.1 Izafet im Ungarischen } & 152\end{array}$

5.2.2.2 Kollokation im Ob-Ugrischen 153

5.2.3 Semantisch motivierte Mechanismen 155

$\begin{array}{lr}\text { Schluss } & 159\end{array}$

$\begin{array}{ll}\text { Verzeichnis der Abkürzungen und Glossierungen } & 167\end{array}$

$\begin{array}{ll}\text { Abbildungsverzeichnis } & 169\end{array}$

$\begin{array}{ll}\text { Tabellenverzeichnis } & 170\end{array}$

$\begin{array}{ll}\text { Quellen } & 171\end{array}$ 


\section{Danksagung}

Die Realisierung des Promotionsvorhabens, dessen Ergebnis die vorliegende Arbeit darstellt, wäre ohne die Unterstützung verschiedenster Seiten nicht möglich gewesen. Der Dank der Verfasserin gilt Frau Professor Elena Skribnik, Herrn Professor Wolfgang Schulze und Herrn Professor Ulrich Schweier für die Annahme und Betreuung des Promotionsvorhabens, der Universität Bayern e.V. für die finanzielle Unterstützung und Modern Academic Publishing für Annahme und Betreuung der Publikation. Das Promotionsvorhaben erfolgte im Rahmen des Linguistischen Internationalen Promotionsprogramms (LIPP) - heute Graduiertenschule Sprache und Literatur - Klasse für Sprache (CoL) der LMU München. Die Verfasserin dankt der Koordination der CoL für die die Unterstützung, insbesondere zum Ende der Promotionszeit, den Hochschullehrern für den fachlichen Mehrwert eines interdisziplinären Promotionsprogrammes und den Mit-Promovierenden für die Gemeinschaftlichkeit. Darüber hinaus gilt den gesamten Mitarbeitern des Institutes für Finnougristik/Uralistik der LMU München inklusive der ehemaligen Mitarbeiter ein herzliches Dankeschön für Beistand, Unterstützung und das angenehme Forschungsklima.

Besonders bedanke ich mich bei meiner Familie und meinen Freunden, die mich auf dem langen und nicht immer einfachen Weg zur Fertigstellung dieser Arbeit begleitet, motiviert und unermüdlich unterstützt haben, und den Electric Boys, Ringo Franco \& Co. für den Soundtrack dazu. 



\section{English Summary}

\section{Possessive suffixes and their functions in Ugric languages}

The Ugric languages Mansi, Khanty and Hungarian form a branch of the Uralic language family which is mainly spread across North-Eastern Europe and Siberia. Other prominent languages of the Uralic family are e.g. Finnish, Saami and Estonian. The Ob-Ugric languages Mansi and Khanty are spoken in Western Siberia along the Ob' river and its tributaries, thus they are referred to as Ob-Ugric. Their closest relative is Hungarian, spoken in Hungary and its neighboring countries. The status of the Khanty and Mansi languages is endangered: only 20\% out of 8,000 ethnic Mansi and 30\% out of 22,000 ethnic Khanty still speak their mother tongue, and there are nearly no monolingual speakers. In contrast, Hungarian is an official language of the European Union, spoken by about 15 million people. Hence, the status of literacy, language documentation and language education differs noticeably between Ob-Ugric and Hungarian.

From a typological point of view, the Ugric languages are basically so-called SOV languages, their morphology is mainly agglutinative, i.e. grammatical information is rather encoded with suffixes which are attached to the stem instead of using prepositions, pronouns or articles. The most accessible referent in a discourse is not overtly realized on the surface of the sentence. Its position remains empty (zero-anaphora). This is also revealed in rich paradigms of personal suffixes which are used instead. One set of personal suffixes is attached to nominal stems and called possessive suffixes. They are involved in the structure of so-called attributive possessive constructions in most Uralic languages. As revealed in their denomination, research on possessive suffixes in Ugric languages, as in most Uralic languages, has primarily viewed them in the light of their function as markers of possessive relations, traditionally referred to as their prototypical use.

The linguistic concept of possession seems to be universal. The notion of possession itself, though, is purely abstract and can only be understood as a »broader concept of association or relationship between two nouns «. While the definition is an abstract collective term, there is a broad consensus among linguists that certain prototypical meanings are covered by the concept of possession. These are: part-whole relations, kinship relations (both by blood and marriage), ownership relations as well as a fourth column covering all kinds of association in general (e.g. attribution, properties or orientation/ location). The use of attributive possessive constructions is very frequent in most Uralic languages and, in a considerable amount of cases, a possessive reading of the relation is excluded, even in the most abstract interpretation of possession. Such cases, where the so-called prototypical use of possessive suffixes (i.e. denoting a possessive relation) fails to serve as an explanation, are frequently subsumed under the node of non-prototypical use and a secondary, non-possessive function is attributed to possessive suffixes. This secondary function is for instance likened to the properties of a definite article.

In my eyes, this approach does not satisfy the features of possessive suffixes in many aspects: there is no inherent possessive meaning, it is the default-interpretation of the relation expressed with a possessive construction and often based on cultural and social 
values. If they limit the use of the construction in one language, this is not necessary the case in other languages, too. Possessive suffixes are morphological markers used in constructions denoting relations traditionally referred to as possession. In consequence, there is no such prototypical use of possessive suffixes and thus no secondary function. Instead, both, the prototypical and the non-prototypical use is an outcome of the very same property of possessive suffixes: as a set of personal markers, they establish reference.

The aim of my study is thus to provide a semasiology-based description of possessive suffixes in Ugric languages. The interpretation of the relation expressed is not the focus. An investigation of possessive suffixes as markers of definiteness is not intended. Not only the occurrence of the possessive suffix, but also the realization of the referent encoded (noun, pronoun or zero anaphora), is considered. The constructions are not analyzed in isolation but in the context of the whole discourse.

Although the majority of data represents the third person singular possessive marker, the whole paradigm is subject to investigation. All nominal stems are considered: while the possessive suffix is in most constructions attached to a noun, other constructions with possessive suffixes, such as verbal nouns and postpositions are also part of the analysis.

The work is corpus-based and mainly framework-free. The theoretical background is limited to terminology: the cognitivists' terms »reference-point", »reference-point construction" and "target « are employed in order to avoid the biased terms "possessor" and "possessee«. Since referentiality is considered as the crucial aspect for the use of possessive suffixes, the principles of information structure are applied. The results might serve as a starting point for further reanalysis and reinterpretation of possessive suffixes and the denomination used to describe them. 


\section{Einleitung}

Die vorliegende Arbeit befasst sich mit Funktionen von Possessivsuffixen in den ugrischen Sprachen. Den ugrischen Sprachzweig der uralischen Sprachfamilie bilden das Ungarische sowie die beiden ob-ugrischen Sprachen Chantisch und Mansisch, welche in Nordwestsibirien entlang des Ob gesprochen werden. Unter Possessivsuffixen versteht man ein Set von Personalmarkern, welches aus Personalpronomen entstanden ist und nominalen Wortstämmen angefügt wird. Wie bereits durch die Terminologie suggeriert sind Possessivsuffixe bislang primär von einem onomasiologischen Gesichtspunkt aus betrachtet worden - als Bestandteil von Possessivkonstruktionen. Bei attributiven Possessivkonstruktionen wird die mithilfe des Possessivsuffixes ausgedrückte Relation dann zum Beispiel als Besitzverhältnis (Beispiel 01), Teil-Ganzes-Relation (Beispiel 02) oder Verwandtschaftsbeziehung (Beispiel 03) interpretiert. Hinzu kommen Belege, welche als nicht näher bestimmte Assoziation jeglicher Art bezeichnet werden (Beispiel 04). ${ }^{1}$

$\begin{array}{llllll}\text { (01) } & \text { SK_text_003_0221:2 } & & & \\ t^{\prime} u: & \text { tfe:moton } & -\partial \phi & t^{j} \partial & \beta ə j & -t \partial \gamma \\ \text { that } & \text { suitcase } & -S G<3 S G & \text { so } & \text { take } & -S G<3 S G\end{array}$

'She took her suitcase with her.'

(02) NM_text_004_0029:2

kol ala te as -in -ir je:mt -əs

house roof $-S G<3 S$ hole $-A D J Z R$-TRNS become -PST[3SG]

'Das Dach des Hauses wurde löchrig.'

(03) KK_text_010_0082

$\begin{array}{lllll}\text { ope } & -m & \text { pox } & \text { taji } & - \text { - } \\ \text { elder sister } & -S G<1 S G & \text { son } & \text { give birth to } & \text {-PST[3SG] }\end{array}$

'Meine Schwester hat einen Sohn geboren.'

(04) HU_text_010_0036

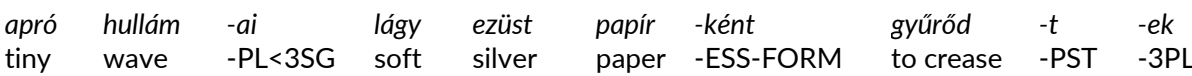

'Seine winzigen Wellen knitterten wie weiches Silberpapier.'

Doch selbst diese Interpretation von Possession im abstraktesten Sinne kann nicht für sämtliche Belege herangezogen werden, wie Beispiel (05) verdeutlicht. Formal handelt es sich hier ebenfalls um eine attributive Nominalphrase mit Possessivsuffix, allerdings handelt es sich hier nicht um eine Possessivkonstruktion im herkömmlichen Sinne. Diese würde nämlich die Relation als Verwandtschaftsverhältnis interpretieren, tatsächlich geht es hier jedoch um eine Relation, welche durch den gemeinsamen vorangehenden Handlungsverlauf entstanden ist.

$1 \mathrm{Zu}$ den Kern-Relationen der Possession siehe Kapitel 1.2.2.1. 
(05) NM_text_007_0024:1

$\begin{array}{lllllll}\text { alpal } & a k w & \text { Ta } & \text { ojka } & -r i s^{j} & -e & -n \\ \text { in the morning } & \text { one } & \text { EMPH1 } & \begin{array}{l}\text { old man } \\ \text { old }\end{array} & \text {-DIM } & \text {-SG<3SG } & \text {-DLAT } \\ \text { nox } & \text { ti } & \text { kwal } & \text {-tt } & \text {-awe } & & \\ \text { up } & \text { EMPH2 } & \text { get up } & \text {-CAUS } & \text {-PASS[PRS.3SG] }\end{array}$

'Am Morgen wurde er von dem kleinen Mann geweckt.'

Hinzu kommen bestimmte Konstruktionen des Ungarischen, in welchen sowohl der für gewöhnlich als Possessor bezeichnete als auch der für gewöhnlich als Possessum bezeichnete Bestandteil der Konstruktion so abstrakt ist, dass die Bestimmung einer Relation im Sinne einer Possessivkonstruktion kaum möglich ist. ${ }^{2}$

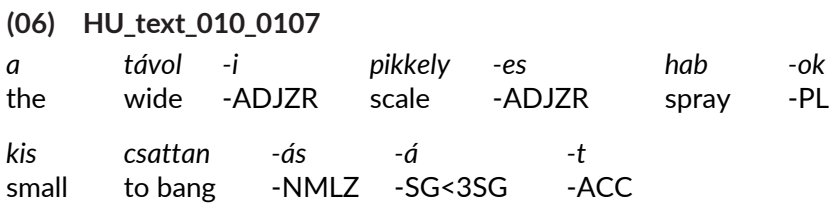

'Die kleinen Schläge der entfernten schuppigen Gischt.'

Für diejenigen der Possessivkonstruktionen (attributive Nominalphrasen mit Possessivsuffix), welche auf Grundlage der Semantik des Lexems, das den Kopf der Konstruktion bildet, nicht als Possessivkonstruktion angesehen werden können, hat sich inner- und außerhalb der Uralistik ein weiterer Forschungsansatz etabliert, welcher Possessivsuffixen, und insbesondere dem der dritten Person Singular, eine zweite, einem definiten Artikel ähnliche Funktion attestiert, zum Beispiel in Form einer anaphorischen Verwendung ${ }^{3}$ (Beispiel 07). Dadurch hat sich gewissermaßen eine Zweiteilung in possessive und nicht-possessive Funktionen der Possessivsuffixe etabliert. ${ }^{4}$

(07) Šuryškary-Chantisch, Simonenko 2014: $4^{5}$

\begin{tabular}{|c|c|c|c|c|c|c|}
\hline $\begin{array}{l}\text { ank } \\
\text { mother }\end{array}$ & $\begin{array}{l}-\partial m \\
-S G<1 S G\end{array}$ & $\begin{array}{l}\text { mulhatd } \\
\text { yesterday }\end{array}$ & $\begin{array}{l}\text { lut } \\
\text { buy }\end{array}$ & $\begin{array}{l}-\partial \\
-P\end{array}$ & $\Gamma[3 S G]$ & $\begin{array}{l}\text { xut } \\
\text { fish }\end{array}$ \\
\hline $\begin{array}{l}\text { xul- } \\
\text { fish }\end{array}$ & $\begin{array}{l}\partial \phi \\
-S G<3 S G\end{array}$ & $\begin{array}{l}\text { tamhatd } \\
\text { today }\end{array}$ & & $\begin{array}{l}\text { le } \\
\text { eat }\end{array}$ & $\begin{array}{l}-S \\
\text {-PST }\end{array}$ & $\begin{array}{l}-i \\
-P A S S[3 S G]\end{array}$ \\
\hline
\end{tabular}

'Yesterday my mother bought a fish. Today we ate this fish.' (wörtlich 'This fish was eaten today.')

Andere Konstruktionstypen mit Possessivsuffixen werden aufgrund der Fokussierung auf den onomasiologischen Ansatz im Großteil der Beschreibungen nur am Rande erwähnt, da weder der Ansatz der Possession noch der Definitheit für die Beschreibung von Konstruktionen mit Verbalnomina oder Postpositionen herangezogen werden kann. Postpositionen sind Synsemantika, somit entfällt die Interpretation einer Besitzrelation (Beispiel 08). ${ }^{6}$ Bei Konstruktionen mit Verbalnomina verleitet die Nominalisierung der Verbalhandlung zur Interpretation als Besitzverhältnis, was jedoch die tatsächliche Funktion der Konstruktion - die Subordination - größtenteils

2 Zu den sogenannten Izafet-Konstruktionen siehe Kapitel 5.2.2.1.

3 Zur Definitheit und zu Possessivsuffixen siehe Kapitel 3.4.

4 Zum Forschungstand siehe Kapitel 2.

5 Beispiele, welche nicht aus dem Korpus der vorliegenden Arbeit stammen, wurden ebenfalls in IPA transliteriert und die Glossierung angepasst, sofern nicht anders angegeben. Zu Notation und Glossierung siehe Kapitel 4.

6 Zur Konstruktion PostP-Px siehe Kapitel 5.1.3. 
unberücksichtigt lässt (eine Nebensatzkonstruktion in Funktion des direkten Objektes wie in Beispiel (09) ist nur in wenigen Fällen belegt, wohingegen die Funktion als temporal-adverbiale Ergänzung sehr viel häufiger vorkommt). ${ }^{7}$

(08) KK_satz_0011

$\begin{array}{lllllll}\text { IKB } & m a: & x^{i} a & -m & -a & j i & -i \\ \text { 3SG } & \text { 1SG } & \text { at } & -1 S G & \text {-DLAT } & \text { come } & - \text { PRS[3SG] }\end{array}$

'Er kommt zu mir.'

(09) SK_text_002_0071:1

$\begin{array}{lllllllll}\text { u: } & \text { torəm } & \text { mən } & -m & -\mathrm{pm}[\ldots] & \text { əntə } & \beta u: & -j & - \text {-əm } \\ \text { lower } & \text { heaven } & \text { go } & \text {-PTCP.PST } & -1 \mathrm{SG} & \text { not } & \text { see } & - \text { PRS } & -1 S G\end{array}$

'I did not see my entering the lower heaven.'

Possessivsuffixe tragen demnach nicht die Bedeutung "possessiv« in sich; mit anderen Worten, die possessive Lesart der Konstruktion wird nicht mittels der Possessivsuffixe ausgedrückt. Sie sind vielmehr Personalmarker, welche die Relation zwischen dem Referenten, dem sie angefügt werden, und der Person, auf die sie referieren, anzeigen. Die Interpretation dieser Relation als possessiv ist eine individuelle und nicht selten von sozio-kulturellen Faktoren abhängige Ermessenssache, die referentielle Verweiskraft der Possessivsuffixe ist davon unabhängig, ob die Lesart eine possessive oder eine andere ist. Die vorliegende Arbeit versucht demnach, Possessivsuffixe von einem semasiologischen Standpunkt aus zu beschreiben, das heißt die Possessivsuffixe anhand ihrer Vorkommen zu kategorisieren und nicht die Ausdrucksmöglichkeiten von Possessivverhältnissen mithilfe von Possessivsuffixen $\mathrm{zu}$ beschreiben.

Ziel der vorliegenden Arbeit ist es daher, Funktionen von Possessivsuffixen in den ugrischen Sprachen ausgehend von ihrer Referentialität zu beschreiben, das heißt, die referentielle Verweiskraft der Possessivsuffixe als Personalmarker dient als Ausgangspunkt der Analyse. Demzufolge soll das gesamte Paradigma der Possessivsuffixe berücksichtigt werden, den Formen des Einbesitzes und insbesondere der dritten Person Singular soll dabei aber besondere Aufmerksamkeit geschenkt werden. Die Lesart der Konstruktion, welche bislang im Fokus der Beschreibungen von Possessivsuffixen stand, ist dabei weitgehend untergeordnet. Stattdessen sollen Nominalphrasen mit Possessivsuffixen nicht als isolierte Einheiten, sondern in Relation zur gesamten Textumgebung beschrieben werden, wobei ebenfalls mit in Betracht gezogen wird, welche Referenten mithilfe der Konstruktion zueinander in Bezug gesetzt werden, sowie deren (syntaktische und pragmatische) Rolle im gesamten Textverlauf. Im Weiteren werden die Bestandteile der Konstruktion, das heißt die Realisierung des im Possessivsuffix kodierten Referenten in der Konstruktion bei der Analyse mit in Betracht gezogen. Erst danach wird die semantische Relation beider in der Konstruktion vorkommenden Referenten analysiert. Dabei geht es jedoch weniger um die Interpretation der Relation, sondern vielmehr um die semantischen Rollen der Referenten in etwa in Hinblick auf ihre Belebtheit. Die Zweiteilung von possessiven und nicht-possessiven Funktionen aufgrund der Semantik des Referenten, welcher den Kopf der Konstruktion bildet, soll dabei aufgehoben werden. Zu einem geringeren Umfang bezieht dieser Ansatz die 
anderen Konstruktionsarten wie Verbalnomina (VN-Px) und Postpositionen mit Possessivsuffixen (РоsтP-Px) mit ein, was nicht zuletzt in der begrenzten Datenmenge begründet liegt. ${ }^{8}$ Diese werden daher in Relation zur attributiven Nominalkonstruktion analysiert.

Der Einblick soll in Form eines kontrastiven Vergleichs erfolgen, einerseits zwischen dem Ungarischen und den ob-ugrischen Sprachen sowie innerhalb des ob-ugrischen Sprachzweigs zwischen dem Mansischen und dem Chantischen. Damit fokussiert die vorliegende Arbeit folgendes Erkenntnisinteresse:

Welche Funktionen üben Possessivsuffixe in den ugrischen Sprachen anhand ihrer referentiellen Verweiskraft hinsichtlich der Strukturierung im Satz sowie im gesamten Text aus?

Ausgangspunkt dabei ist die Annahme, dass sich die referentielle Verweiskraft je nach Konstruktionsart und kodierter Person soweit verändert, dass unter Umständen von mehr als einer Kategorie Possessivsuffix gesprochen werden sollte und die Glossierung dementsprechend angepasst werden muss. So ist zum Beispiel die Kodierung von zwei Referenten ausschließlich bei Substantiven gegeben, bei Konstruktionen mit Verbalnomina und Postpositionen wird hingegen nur ein Referent kodiert und nur ein Teil des Paradigmas verwendet (formal ist dieses gleich mit dem des Einbesitzes, jedoch muss hier unterschieden werden zwischen Markern, welche zwei Referenten kodieren, und Markern, welche nur einen Referenten kodieren). Es wird weiterhin angenommen, dass die Possessivsuffixe aller drei ugrischen Sprachen aufgrund des gemeinsamen Ursprungs über gemeinsame Verwendungen und Funktionen verfügen, die eine Art ugrischen Kern bilden. Darüber hinaus weisen Funktionen und Verwendung der Possessivsuffixe in den ugrischen Sprachen Unterschiede auf, wobei sich insbesondere zwischen dem Ungarischen und den ob-ugrischen Sprachen eine Entwicklung in zwei entgegengesetzte Richtungen bemerken lässt: Während im Ungarischen vor allem das Possessivsuffix der dritten Person Singular einen konstruktiven Charakter, das heißt zu einem Linker zwischen zwei Substantiven innerhalb einer Konstruktion, angenommen hat und damit Teile bzw. seine gesamte referentielle Verweiskraft verschwunden ist, ist die Entwicklung in den ob-ugrischen Sprachen eher in informations-strukturelle Bereiche erfolgt, wo ebenfalls insbesondere das Possessivsuffix der dritten Person Singular häufig zum Einsatz kommt. Entgegen diesen ersten Hypothesen hat sich im Verlauf der Analyse jedoch herausgestellt, dass diese Zweiteilung weitaus geringer ausgeprägt ist und auch im Ungarischen zumindest in dem für die Analyse ausgewählten Textgenre die Possessivsuffixe ähnliche text-strukturierende Funktionen aufweisen wie im ObUgrischen. ${ }^{9}$ Diese Erkenntnis steht im Einklang damit, dass im überwiegenden Teil der Konstruktionen Subs-Px der im Possessivsuffix kodierte Referent als Leerstelle realisiert wird (knapp drei Viertel der Belege) ${ }^{10}$ - das Possessivsuffix setzt damit in allen drei Sprachen als Pro-Form eine hohe Topikalität voraus. Entsprechend den ursprünglichen Erwartungen sind die am häufigsten vorkommenden Possessivsuffixe die der dritten Personen, und insbesondere das der dritten Person Singular Einbesitz. In Hinsicht auf

$8 \mathrm{Zu}$ den Daten siehe Kapitel 4.1.

$9 \mathrm{Zu}$ den Konstruktionen und ihrer Textumgebung, siehe Kapitel 5.2.

10 Zur Methodik siehe Kapitel 4.2. 
das der Analyse zugrunde liegende Korpusmaterial ${ }^{11}$ - Texte, hauptsächlich Narrationen - ist jedoch die Anzahl der Vorkommen von Possessivsuffixen der ersten und zweiten Person erwähnenswert (beinahe ein Drittel der Belege).

Die Ergebnisse der Datenanalyse der vorliegenden Arbeit lassen die Idee eines Kontinuums entstehen, in welchem die Funktionen zwischen pragmatisch motivierten (Verankerung eines neuen, unzugänglichen Referenten, Referentenwechsel etc.) und syntaktisch motivierten Mechanismen (als Marker der Zugehörigkeit von Modifizierer zum Kopf) verteilt werden könnten.

Die Arbeit setzt sich wie folgt zusammen: Kapitel 1 gibt einen Einblick in den Forschungsgegenstand; hier werden sowohl die behandelten Sprachen samt kurzer Übersicht der dialektalen Gliederung und Skizzierung der Sprachsituation beschrieben (Teilkapitel 1.1) als auch die Paradigmen der Possessivsuffixe aller drei Sprachen vorgestellt (Teilkapitel 1.2). Das Teilkapitel 1.2 beinhaltet auch eine Diskussion zur Terminologie -»Possessivsuffix «, Ausdruckvon»Possession «, Possessivkonstruktion «und eine Abgrenzung des Forschungsgegenstands gegenüber den anderen Bereichen der linguistischen Dimension »Possession«. Kapitel 2 widmet sich dem Forschungsstand und bietet einen Überblick zur bisherigen Forschung zum Possessivsuffix, sowohl innerhalb als auch die Reflexion außerhalb der Uralistik. Ein Teil des Kapitels widmet sich dabei der Forschung zur Definitheit im Zusammenhang mit den Possessivsuffixen des Uralischen. Kapitel 3 skizziert die theoretischen Grundlagen der vorliegenden Arbeit, wobei diese als vorwiegend framework-free ${ }^{12}$ zu betrachten ist. Der theoretische Rahmen beschränkt sich daher auf die Beschreibung des theoretischen Hintergrundes der zur Analyse gewählten Methodik und auf die Definition der verwendeten Terminologie. Bei der Wahl des theoretischen Rahmens standen Forschungsansätze, welche den in der vorliegenden Arbeit fokussierten Aspekt der Referentialität betreffen, im Vordergrund: die Functional Grammar, insbesondere im text-linguistischen Sinne, und die Kognitive Linguistik, und zwar dort vorwiegend die Referenzpunkt-Konstruktionen.

Kapitel 4 stellt die zur Analyse des Forschungsgegenstandes gewählte Methodik vor, die Vorgehensweise bei der Datenanalyse, die Daten und die Datenerhebung selbst. Kapitel 5 legt den Beitrag der vorliegenden Arbeit zum Erkenntnisgewinn dar und beinhaltet die Ergebnisse und Diskussion. Der erste Teil stellt dabei die einzelnen Konstruktionen vor (Kapitel 5.1.1-5.1.3) und beschreibt sie aufgrund der Vorkommen im Korpus. Der zweite Teil (Kapitel 5.2) bezieht exemplarisch die Textumgebung in die Analyse mit ein und beschreibt die Auswirkungen der Possessivkonstruktion auf die Strukturierung von Satz und Text. Text-Strukturierung wird in der vorliegenden Arbeit dabei als die Strukturierung, die durch das referentielle Verweismittel Possessivsuffix im Text erfolgt, verstanden. Diese kann zum einen anaphorisch, das heißt rückverweisend, erfolgen, indem das Possessivsuffix als Pro-Form im Text einen topikalen Referenten kodiert, oder deiktisch, indem das Possessivsuffix auf einen bestimmten Referenten hinweist. In anderen Fällen hingegen ist der Antezedens, das heißt die letzte Vorerwähnung des Referenten, innerhalb derselben Konstruktion zu finden. Auch in diesen Fällen erfolgt durch die Markierung des Possessivsuffixes eine Strukturierung: Es wird die 
Zugehörigkeit des Modifizierers zum Kopf der attributiven Nominalphrase angezeigt. Die Analyse berücksichtigt dabei auch in der Fachliteratur beschriebene Mechanismen von Possessivkonstruktionen und ordnet sie anhand der Korpusdaten für die ugrischen Sprachen ein. Kapitel 6 bildet den Schluss, stellt eine Zusammenfassung aus den Erkenntnissen dar und gibt einen Ausblick auf weitere mögliche Forschungsaspekte.

Es wird versucht, den Forschungsgegenstand weitestgehend in einer neutralen bzw. einer Terminologie zu beschreiben, die frei von Bezügen auf Possession ist. Der Terminus »Possessivsuffix» selbst soll jedoch nicht neu benannt werden. 


\section{Forschungsgegenstand}

\subsection{Die ugrischen Sprachen}

Die vorliegende Arbeit befasst sich mit Funktionen von Possessivsuffixen in ugrischen Sprachen. Hierzu zählen das Ungarische (mit ISO 639-3 code »hun «) ${ }^{13}$ sowie die beiden ob-ugrischen Sprachen Chantisch (mit ISO 639-3 code »kca«, ältere und Fremdbenennung Ostjakisch) und Mansisch (mit ISO 639-3 code »mns«, ältere und Fremdbenennung Wogulisch). Gemeinsam bilden alle drei Sprachen den ugrischen Zweig der uralischen Sprachfamilie. Die Trennung zwischen Ungarisch und ObUgrisch erfolgte schon ungefähr im fünften bis siebten Jahrhundert v. Chr. (Keresztes 1998: 389), sodass zwar heute noch gemeinsame typologische Züge und Ähnlichkeiten im Grundwortschatz auszumachen sind, eine gegenseitige Verständigung - auch nicht innerhalb der ob-ugrischen Sprachen - jedoch nicht möglich ist.

\subsubsection{Typologie der ugrischen Sprachen}

Die ugrischen Sprachen sind - wie die meisten uralischen Sprachen - typologisch gesehen vorwiegend agglutinierende SOV-Sprachen mit einer entsprechenden Vielzahl von Suffixen. Aufgrund der Vokalharmonie im Ungarischen und in einigen ob-ugrischen Dialekten sowie prosodisch bedingten Vokalwechseln bzw. -reduktionen weisen die Suffix-Paradigmen dazu eine Vielzahl an Allomorphen auf (verglichen Comrie 1988: 452f.).

Die nominalen Kategorien sind Numerus (Singular und Plural im Ungarischen, zusätzlich Dual im Ob-Ugrischen), Kasus und Person; letztere wird durch das Paradigma der Possessivsuffixe ausgedrückt. Die Anzahl der Kasussuffixe unterscheidet sich von Sprache zu Sprache und sogar dialektal: Während dem Ungarischen je nach Definition bis zu 31 Kasus attestiert werden, weist zum Beispiel das Nord-Chantische lediglich drei Kasus auf (zu den typologischen Besonderheiten und Unterschieden der chantischen Dialekte siehe Kapitel 1.1.2 Die ob-ugrischen Sprachen). Syntaktische Kernrelation werden jedoch eher durch Wortstellung und Agreement am Verb markiert als durch Kasussuffixe, sodass syntaktische Kasus in den ugrischen Sprachen kaum vorhanden sind (verglichen Comrie 1975). Der ur-ob-ugrische Akkusativ - $m$ ist beispielsweise nur noch im Ost-Mansischen zu finden, in den anderen ob-ugrischen Dialekten ist er verschwunden (Honti 1998: 343f.). Der ungarische Akkusativ auf - $t$ hat ebenfalls keinen gemeinsamen Ursprung im Ur-Ugrischen, sondern entwickelte sich aus einem klitisierten Demonstrativpronomen (Abondolo 1998a: 441).

Bleiben die syntaktischen Kernrollen somit relativ unmarkiert im ugrischen Satz, werden insbesondere lokal-adverbiale Bestimmungen mit einer Vielzahl an morphologischen und morpho-syntaktischen Markierungen versehen (verglichen Comrie 1988: 462f.). Neben zahlreichen Kasussuffixen, vor allem im Ungarischen, kommt hier ein vielfältiges System von Postpositionen (Adpositionen, die dem Bezugswort folgen) zum Einsatz, welche ebenfalls paradigmatisch mit Kasussuffixen versehen werden können, 
zum Beispiel Ungarisch: alatt ,unter, alá ,nach unten', alól ,von unten'. Postpositionen können ebenfalls mit Possessivsuffixen versehen werden, siehe dazu Kapitel 5.1.3. Neben lokalen Bestimmungen können insbesondere im Chantischen mit postpositionalen Konstruktionen auch andere adverbiale Bestimmungen, oft in Kombination mit infiniten Verbalformen (temporal, kausal, konditional), ausgedrückt werden. Eine detaillierte Analyse darüber findet sich bei Schön 2014.

Die verbalen Kategorien umfassen unter anderem Person, Numerus (ebenfalls im Dual im Ob-Ugrischen), Tempus (im Ungarischen gibt es eine Vergangenheitsform, in den Ob-Ugrischen Sprachen bis zu vier, Futur gibt es in keiner ugrischen Sprache als synthetisch gebildetes Tempus), Modus und zu einem gewissen Grad auch Aspekt (insbesondere im Ungarischen mithilfe von Verbalpräfixen ausgedrückt) sowie Genus Verbi. Die Negation wird in allen drei Sprachen mittels Negationspartikeln ausgedrückt, welche sich je nach Modus unterscheiden können. Die ob-ugrischen Sprachen verfügen außerdem über eine Distinktion zwischen Realis und Irrealis in Bezug auf die Äußerung: Für tatsächlich von ihm bezeugte Sachverhalte benutzt der Sprecher den Indikativ, für Sachverhalte, welche er durch Hörensagen über Dritte oder Inferenz/Deduktion wahrgenommen hat, oder um eigenes Überraschen (»unprepared mind «) auszudrücken, benutzt er den Mirativ (im Chantischen ist eher die Bezeichnung Evidentialis gebräuchlich; die Kategorie und ihre Verwendung ist in keiner der beiden ob-ugrischen Sprachen bislang abschließend erforscht, siehe hierzu auch Kapitel 5.1.2.2). Alle drei Sprachen kodieren am Verb nicht nur Person und Numerus des Subjektes, sondern unter bestimmten Bedingungen auch den Numerus des direkten Objektes. Dementsprechend verfügen alle drei Sprachen über zwei Paradigmen von verbalen Personalmarkern (sogenannte subjektive und objektive Konjugation). Beide Paradigmen haben einen gemeinsamen ur-uralischen Ursprung mit dem Paradigma der Possessivsuffixe in Personalpronomen.

Kopulaverben sind vor allem im Präsens in der dritten Person kaum gebräuchlich und statt Koordination mit Konjunktionen werden häufiger subordinierende Partizipial- und Konverb-Konstruktionen verwendet, welche ebenfalls Possessivsuffixe enthalten können (siehe Kapitel 5.1.2) (verglichen Comrie 1988: 466f.).

Ungarisch ist eine der wenigen uralischen Sprachen, welche einen definiten Artikel vorweist. Dieser wird als sekundäre, kontaktinduzierte Entwicklung angesehen. Eine eher typisch uralische Strategie, die der Definitheit zugrunde liegenden Eigenschaften (zum Beispiel Vorerwähntheit/Topikalität/Salienz/Identifizierbarkeit) zu markieren, ist ein Zusammenspiel von pro-drop, subjektiver und objektiver Konjugation und Differential Object Marking. Darüber hinaus existiert eine starke Korrelation in der Hierarchie von syntaktischen und pragmatischen Rollen, welche zusammen ein System von reference-tracking Mechanismen bilden, in welchen auch Possessivsuffixe eine Rolle spielen. Dies wird in Kapitel 3.2.2 im Detail vorgestellt werden.

\subsubsection{Die ob-ugrischen Sprachen}

Die ob-ugrischen Sprachen Chantisch und Mansisch werden in Westsibirien entlang des Ob und seiner Nebenflüsse gesprochen. Laut dem »UNESCO Weltatlas der bedrohten Sprachen der Welt« (Moseley (Hg.) 2010) sind sämtliche Dialekte beider Sprachen 
als gefährdet anzusehen (die Klassifizierung reicht hier von »definitely« über »severely« bis zu »critically endangered «) (Moseley (Hg.) 2010). Einige Dialekte sind bereits ausgestorben. Insgesamt kann davon ausgegangen werden, dass nur ein geringer Anteil (rund ein Fünftel) derjenigen, die sich zum Volk der Chanten oder Mansen zählen, tatsächlich noch eine ob-ugrische Sprache als Muttersprache spricht - die vorherrschende Sprache ist Russisch, und fast kein Ob-Ugrier spricht ausschließlich eine ob-ugrische Sprache. Laut dem Zensus von $2010^{14}$ wird die Sprecherzahl auf knapp 10.000 Sprecher für alle chantischen Dialekte geschätzt und auf circa 2.000 Sprecher für das Mansische, bei letzteren handelt es sich dabei fast ausschließlich um Sprecher der Nord-Dialekte (Moseley (Hg.) 2010). Der Großteil der Sprecher ist heute über fünfzig Jahre und älter und die Sprache wird oft nicht mehr an die jüngeren Generationen weitergegeben. ${ }^{15}$ Deshalb handelt es sich bei den ob-ugrischen Sprachen und Dialekten ausnahmslos um Minderheitensprachen indigener Völker Sibiriens, welche stark vom Aussterben bedroht sind.

Beide Sprachen sind verschriftlicht, wobei die Schreibtradition in keiner der beiden Sprachen länger als hundert Jahre zurückreicht. Die Schriftsprache des Mansischen basiert hauptsächlich auf den Nord-Dialekten (insbesondere auf dem Sosva-Dialekt, siehe unten) (Keresztes 1998: 390), im Chantischen existiert sowohl eine Schriftvariante der Nord-Dialekte als auch der Ost-Dialekte - dies beruht zum einen darauf, dass ab den 1930er-Jahren, als mit der Schaffung einer Schriftsprache begonnen wurde, mehrere Varianten herangezogen wurden, andererseits aufgrund der dialektalen Unterschiede, die eine einheitliche verschriftlichte Standardsprache nicht zulassen (verglichen Hajdú/ Domokos (Hg.) 1987: 60). Alle Schriftvarianten verwenden heute das kyrillische Alphabet mit einigen Zusatzzeichen. In der Ob-Ugristik hingegen wird zur Notation vorwiegend die Finnisch-Ugrische Transkription (FUT) oder das Internationale Phonetische Alphabet (IPA) gebraucht.

In beiden Sprachen wird seit rund 25 Jahren eine eigene kleinere Zeitung publiziert (chantisch Хӑнmы Ясау und mansisch Лўима̄ Сэрипос) - beide Sprachfassungen sind mittlerweile auch online zugänglich. ${ }^{16}$ Ein Großteil der Publikationen sind Übersetzungen aus dem Russischen, Text- bzw. Lehrbücher oder »kleinere literarische Werke« (Honti 1988a: 175). Auch wenn die Literaturtradition der ob-ugrischen Sprachen damit größtenteils der in allen sibirischen Sprachen nach demselben Muster geschaffenen Universalliteratur entspricht (Hajdú/Domokos (Hg.) 1987: 546f.), darf man dadurch keine Rückschlüsse auf die reiche Erzähltradition beider Völker ziehen. Viele ob-ugrische Erzählungen wurden während Feldforschungsaufenthalten zahlreicher Forscher der ob-ugrischen Sprachen seit mehr als hundert Jahren dokumentiert und liegen oftmals bis heute unpubliziert in linguistischen Transkriptionen vor. Eine detaillierte Beschreibung zur Situation der ob-ugrischen Sprachen und der linguistischen Aufarbeitung bietet hier die gemeinsame Website der internationalen Forschungsprojekte »Ob-Ugrische Sprachen: konzeptionelle Strukturen, Lexikon, Konstruktionen,

14 Detaillierte Angaben zum Zensus siehe Ob-Ugric Database, »Introduction«, http://www.oudb.gwi.unimuenchen.de/index.php?abfrage=ob_ugric\&navi=introduction (Zugriff vom 14.05.2017).

15 Eine Einschätzung zur Lebensfähigkeit (viability) einzelner ob-ugrischer Dialekte findet sich bei Documentation of Eastern Khanty, http://www.policy.hu/filtchenko/Documenting\%20Eastern\%20Khanty/ Eastern\%20Khanty\%20Map.htm (Zugriff vom 14.05.2017).

16 Хӑнты Ясау und Лӯима̄ Сэ̄рипос, http://www.khanty-yasang.ru/frontpage (Zugriff vom 16.05.2017). 
Kategorien« (OUL) und »Ob-Ugrische Datenbanken: Analysierte Korpora und Wörterbücher kaum beschriebener ob-ugrischer Dialekte «(OUDB). ${ }^{17}$

Das Siedlungsgebiet der Ob-Ugrier erstreckt sich über ein verhältnismäßig großes Areal, weswegen sowohl Chantisch als auch Mansisch dialektal stark gegliedert sind. Traditionell werden daher die Dialekte beider Sprachen je nach Siedlungsgebiet anhand der Himmelsrichtungen in drei bis vier Dialektgruppen mit verschiedenen Dialekten unterteilt und gegebenenfalls noch einmal in Subdialekte untergliedert (die vorliegende Arbeit folgt der in Schön 2014 festgelegten Hierarchie und verwendet die Begriffe Dialektgruppe - Dialekt - Subdialekt; verglichen Schön 2014: 11).

Die Bezeichnungen der Dialekte und Subdialekte entsprechen meist den Flussläufen oder Ortschaften, in deren Umgebung sie gesprochen werden.

Der traditionelle Siedlungsraum der Mansen in Nordwest-Sibirien erstreckt sich in etwa im Gebiet zwischen den Osthängen des Urals und den westlichen Zuflüssen des unteren $\mathrm{Ob}^{18}$ (russisch Обь) (Keresztes 1998: 390) sowie an den Nebenflüssen des Irtysch (russisch Иртыш), deren Quellen in diesem Gebiet entspringen. Bis ins 19. Jahrhundert waren außerdem an den Westhängen des Urals Siedlungsgebiete der Mansen zu finden (Keresztes 1998: 390). Administrativ erstreckt sich der Siedlungsraum auf den westlichen Teil des Autonomen Kreises der Chanten und Mansen - Jugra (russisch Ханты-Мансийский автономный округ - Югра) ${ }^{19}$, in welchem der Großteil der Mansen lebt, sowie auf Teile des Gebiets Tjumen (russisch Тюменская область). ${ }^{20}$

Kleinere Gruppen leben im Gebiet Sverdlovsk (russisch Свердловская область) ${ }^{21}$ und im Autonomen Kreis der Jamal-Nenzen (russisch Ямало-Ненецкий автономный округ $)^{22}$ (Murphy 1968: 3; mit Anpassung an die heutigen Bezeichnungen der administrativen Einheiten).

Im Norden und Westen grenzt das Siedlungsgebiet der Mansen an das der KomiSyrjänen, im Norden außerdem an das der Nenzen, im Süden an das der Tataren und im Osten an das der Chanten (Kálmán 1963: 7).

Der Kontakt zu den benachbarten Völkern macht sich insbesondere in der Lexik bemerkbar (Hajdú/Domokos (Hg.) 1987: 56f.). Das Mansische wird in vier Dialektgruppen unterteilt (Nord-, Süd-, West- und Ost-Mansisch) (Keresztes 1998: 390). Die maßgeblichsten Unterschiede ergeben sich in den Vokalsystemen der vier Dialektgruppen (Hajdú/Domokos (Hg.) 1987: 54). Der charakteristische Laut der Ostdialekte /œœ/ entspricht Nordmansisch /aa/ und Südmansisch /ää/ (Keresztes 1998: 390).

Zur nördlichen Dialektgruppe des Mansischen werden die Dialekte von Sygva (am Flusslauf der Sygva, russische Bezeichnung Ляпин), Ob (an den westlichen Ufern,

17 Ob-Ugric Database, http://www.oudb.gwi.uni-muenchen.de/ (Zugriff vom 14.05.2017).

18 Die deutschen Schreibweisen der geografischen Bezeichnungen sind - sofern dort aufgeführt - gemäß Duden (Drosdowski (Hg.) 1996); Begriffe, welche dort nicht aufgeführt werden, wurden gemäß ISO 9/ DIN 1460 transliteriert.

19 Website des Autonomen Kreises der Chanten und Mansen, http://www.admhmao.ru/ (Zugriff vom 14.05.2017).

20 Website des Gebiet Tjumen, http://admtyumen.ru/ogv_ru/index.htm (Zugriff vom 14.05.2017).

21 Website des Gebiet Sverdlovsk, http://www.midural.ru/ (Zugriff vom 14.05.2017).

22 Website des Autonomen Kreis der Jamal-Nenzen, http://правительство.янао.pф/ (Zugriff vom 14.05.2017). 


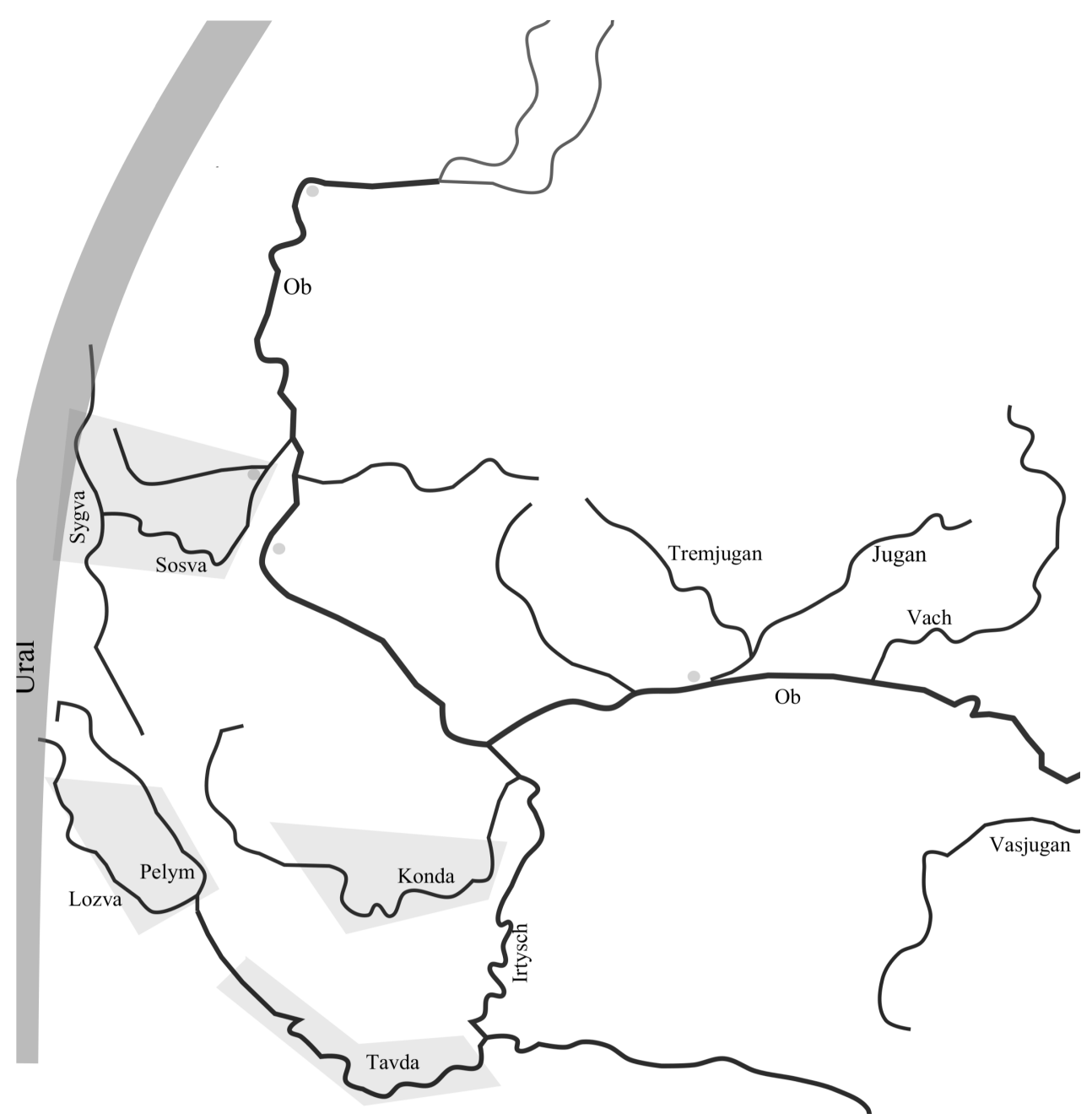

Abbildung 1: Siedlungsgebiete der Mansen, eigene Adaption nach Csepregi 1998: 48.

südlich von Берёзово, Murphy 1968: 1), Obere Lozva (am Flusslauf der Loz'va r. Лозьва) und Sosva (am Flusslauf der Sos'va r. Северная Сосьва) (Hajdú/Domokos (Hg.) 1987: 53; Murphy 1968: 1) gezählt. Die Dialektgruppe ist trotz eines recht großen Verbreitungsgebietes relativ konform, mit geringen Abweichungen in Phonologie und Lexik (Kálmán 1963: 8). Das Gebiet der mansischen Nord-Dialekte grenzt an das der Nord-Dialekte des Chantischen, des Weiteren besteht Sprachkontakt mit dem Komi und dem Nenzischen (Keresztes 1998: 390). Merkmale, welche auf Kontakteinfluss zurückgeführt werden, sind zum Beispiel der Wechsel vom Vorder- zum Hintervokal ( ${ }^{*} /$ ää/ $\left.>/ \mathrm{aa} /\right)$ oder Wechsel vom Lateralapproximant zum Lateralfrikativ $\left({ }^{*} / \mathrm{l} /, / \mathrm{j} />\right.$ $/ 1 /, / 1 \mathrm{j} /)$. Letzterer hat insbesondere im Ob-Dialekt stattgefunden (Honti 1988b: 148), was durch die weite Verbreitung des Lateralfrikativs im Chantischen (Keresztes 1998: 390) und einen dementsprechend kontaktinduzierten Einfluss erklärt werden kann (Honti 1988b: 148). Auch im Bereich der Lexik weichen die Norddialekte stark von den 
anderen Dialektgruppe ab, zum Beispiel NM sa:li WM (Pelym) kunnə OM konуə SM konka: ,Rentier' und NM e:ri WM (Pelym) koårəx OM köärəx SM kärəx, ist nötig' (Honti 1988b: 149; IPA-Transliterierung GJ).

Die Dialekte der Südgruppe wurden bis Anfang des 20. Jahrhunderts entlang der Tavda (r. Тавда) gesprochen (Keresztes 1998: 390) und haben sich mittlerweile - auch durch die isolierte Lage des Dialektes - mit der vorherrschenden tatarischen Sprache assimiliert (Hajdú/Domokos (Hg.) 1987: 53). Bedeutende Subdialekte waren die Varianten der Ortschaften Janyčkova, Čandyri und Gorodok (Honti 1988a: 148 und Honti 1998: 327). In den Süd-Dialekten hatte sich ein Großteil des ur-mansischen Vokalismus sowie die Vokalharmonie erhalten (verglichen Hajdú/Domokos (Hg.) 1987: 55; Honti 1988a: 149).

Der Einfluss des Tatarischen machte sich insbesondere durch die Akzentverlagerung auf die zweite Silbe bemerkbar (Keresztes 1998: 390). Den Komitativ gab es nur in der Süd-Gruppe, der Dual hingegen war in dieser Dialektgruppe verschwunden (Hajdú/ Domokos (Hg.) 1987: 55).

Die Dialekte der westlichen Gruppe unterscheiden sich untereinander in größerem Umfang, wobei die gesamte Dialektgruppe ebenfalls als inzwischen ausgestorben anzusehen ist (Hajdú/Domokos (Hg.) 1987: 53). Zur westlichen Gruppe werden die Dialekte Pelym entlang der Pelymka (r. Пелым), Mittlere Lozva und Untere Lozva entlang des gleichnamigen Flusses Lozva und Vagilsk (weiter unterteilt in Nord- und Süd-Vagilsk) an den Zuflüssen der Tavda gezählt (Murphy 1968: 1). Der ehemals westlich des Urals an der Čusovaja (r. Чусова́я) gesprochene Dialekt wird ebenfalls zur Westgruppe gezählt. Aufgrund der geografischen Nähe war die Beeinflussung durch das Komi »signifikant« (Keresztes 1998: 390). Zu den distinktiven Merkmalen sind ein Schwund des Duals und die Diphthongierung langer Vokale zu zählen (Keresztes 1998: 390).

Die Gruppe der Ostdialekte, bestehend aus den Dialekten an der Konda (r. Конда) - Obere Konda, Mittlere Konda und Untere Konda - sowie Jukonda am gleichnamigen Fluss (r. Юконда) (Murphy 1968: 1; Honti 1998: 327f.), stellten noch bis in die 1920er-Jahre die zweitgrößte Dialektgruppe dar (Hajdú/Domokos (Hg.) 1987: 53). Heute ist die genaue Anzahl an Sprechern nicht mehr bestimmbar, die Dialekte dürften jedoch nahezu ausgestorben sein (verglichen Moseley (Hg.) 2010). Insbesondere die Sprecher an der unteren Konda waren in Kontakt mit Sprechern des Chantischen, außerdem ist der Einfluss des Tatarischen bemerkbar. Auch im Ost-Mansischen ist die Vokalharmonie erhalten geblieben (Keresztes 1998: 390).

\begin{tabular}{llll}
\hline Nordgruppe & Südgruppe & Westgruppe & Ostgruppe \\
\hline Sygva & Tavda & Pelym & Obere Konda \\
Sosva & - Janyčkova & Mittlere Lozva & Mittlere Konda \\
Ob & - Čandyri & Untere Lozva & Untere Konda \\
Obere Lozva & - Gorodok & Vagilsk & Jukonda \\
& & - Nord-Vagilsk & \\
& & -Süd-Vagilsk & \\
\hline
\end{tabular}

Tabelle 1: Dialektale Gliederung des Mansischen. 


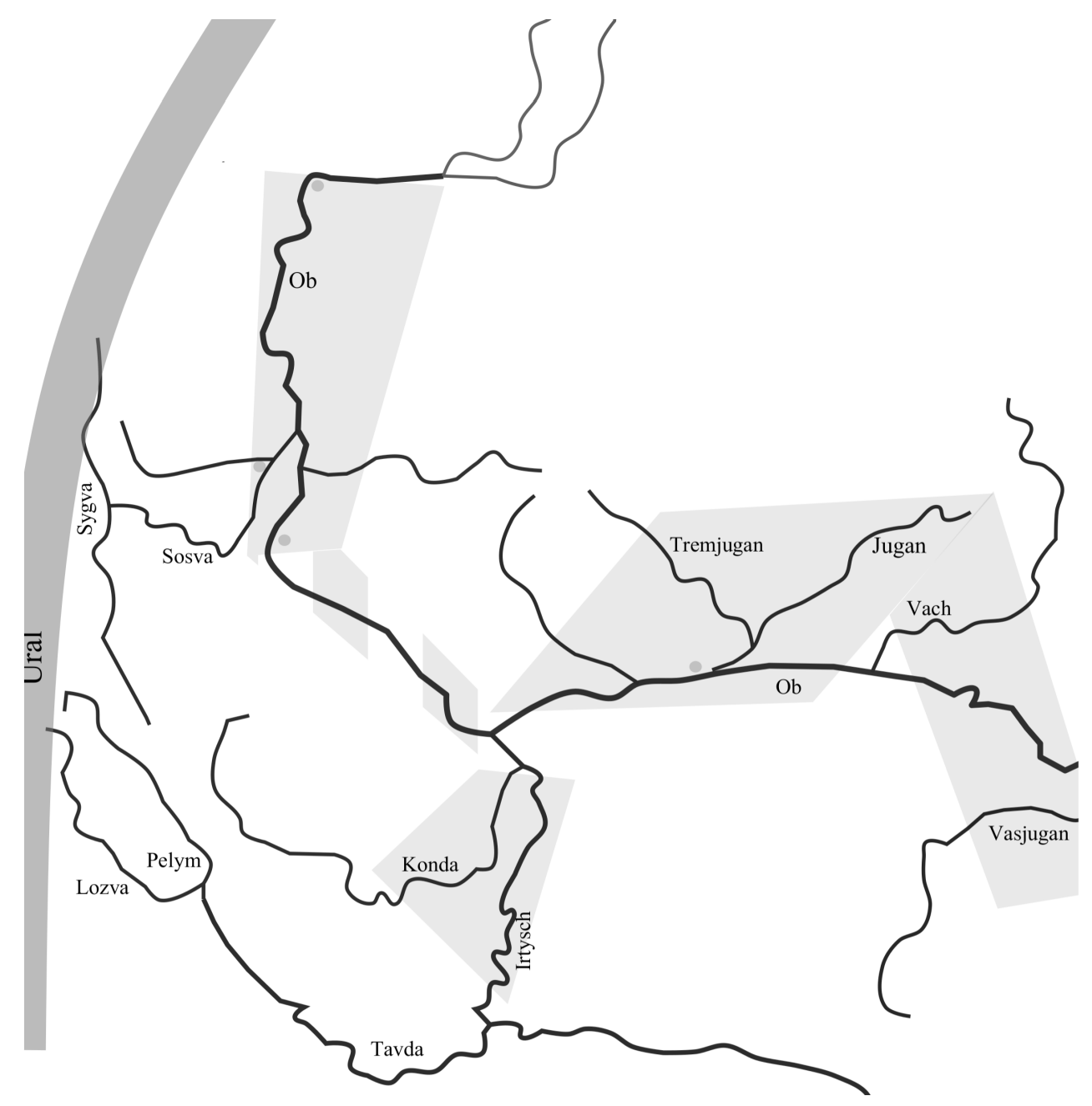

Abbildung 2: Siedlungsgebiete der Chanten, eigene Adaption nach Csepregi 1998: 60.

Das traditionelle Siedlungsgebiet der Chanten liegt östlich dem der Mansen, entlang des $\mathrm{Ob}$ und dessen Zuflüssen sowie entlang des unteren Irtysch (Abondolo 1998c: 358). Administrativ liegt der Siedlungsraum größtenteils im Autonomen Kreis der Chanten und Mansen - Jugra, kleinere Gruppe leben im Autonomen Kreis der JamalNenzen sowie im Gebiet Tomsk (russisch Томская область) (Csepregi 1998/2011: 6; deutsche Bezeichnungen der administrativen Einheiten GJ). Außer dem Siedlungsgebiet der Mansen - von den Chanten aus gesehen im Westen - grenzt im Nordwesten das der Tundra-Nenzen und Komi-Syrjänen an das der Chanten, im Osten das der Wald-Nenzen und Selkupen, im Süden das der Tataren; mit allen Nachbarvölkern besteht sprachlicher und kultureller Kontakt (Csepregi 1998/2011: 6).

Aufgrund des vergleichsweise großen Siedlungsgebietes unterscheiden sich die Dialekte des Chantischen mitunter stark auf phonologischer, morphologischer und syntaktischer Ebene (Rédei 1965: 4). Somit können sich Sprecher verschiedener Dialekte auch 
innerhalb einer Dialektgruppe nicht zwangsläufig gegenseitig verständigen (Schön 2014: 9f.). Die Systematisierung der chantischen Dialekte erfolgt für gewöhnlich anhand der morphologischen Unterschiede (Rédei 1965: 4), manche Systematisierungen ziehen auch die lautliche Entwicklung aus dem Ur-Chantischen heran (Honti 1988a: 173). Damit werden die chantischen Dialekte traditionell in drei Hauptgruppen unterteilt: in Nord-, Süd- und Ost-Dialekte; gelegentlich werden auch die Nord- und SüdDialekte noch einmal zu einer Westgruppe zusammengefasst (Schön 2014: 16). Dennoch sind die Grenzen, wo eine Dialektgruppe endet und wo die nächste beginnt, nicht immer einheitlich zu ziehen und beruhen oft darauf, welche linguistischen Merkmale anderen vorgezogen werden. Die markanteste Abweichung ergibt sich in der Anzahl der Kasus und Vergangenheitstempora, welche von Ost nach West abnimmt: In den Ost-Dialekten gibt es bis zu elf Kasus, im Süden bis zu sechs, während im Nord-Dialekt nur drei Kasus verwendet werden (Hajdú/Domokos (Hg.) 1987: 64). Im Osten sind hingegen bis zu vier Vergangenheitstempora belegt, im Norden und Süden jeweils eines (Hajdú/Domokos (Hg.) 1987: 64). Der bemerkenswerteste phonologische Unterschied zwischen dem Süd- und den Nord- sowie teilweise den Ostdialekten ist die Opposition von /t/ und /l/ aus ur-chantisch */l/ (Hajdú/Domokos (Hg.) 1987: 62).

In der vorliegenden Arbeit soll eine möglichst allgemeingültige Einordnung nach Dialektgruppen gegeben werden, welche eine erste Orientierung ermöglichen soll und die in der vorliegenden Arbeit behandelten Dialekte einordnet. Eine detaillierte Gliederung der chantischen Dialekte inklusive Einordnung von Subdialekten und Forschungsgeschichte zur Dialektologie des Chantischen findet sich in Schön 2014.

Die Norddialekte des Chantischen werden von der Ob-Mündung bis zum Zusammenfluss von Ob und Irtysch gesprochen (Csepregi 1998/2011: 6); manchmal wird die Südgrenze auch etwas nördlicher angesetzt, bei der Landgemeinde Šerkaly (r. сельское поселение Шеркалы) (Rédei 1965: 4, Hajdú/Domokos (Hg.) 1987: 60) oder bei Nizjam (Schön 2014: 17). Zur nördlichen Dialektgruppe werden die Dialekte Obdorsk ${ }^{23}$, MittelOb, Kazym, Berëzovo und Šuryškary gezählt (Schön 2014: 43). Bedeutendere Subdialekte sind zum Beispiel die Subdialekte Nizjam, Keuši und Šerkaly des Mittel-Ob-Dialektes oder die Subdialekte Muži und Synja des Šuryškary-Dialektes (Schön 2014: 43). Der Sygva-Dialekt ist nur in Antal Regulys Aufzeichnungen zu finden und ist vermutlich schon Ende des 19., spätestens Mitte des 20. Jahrhunderts ausgestorben (Schön 2014: 31, Rédei 1965: 95). Der Obdorsker, der Šuryškary-Dialekt und der Šerkaly-Subdialekt sind Basis der nord-chantischen Schriftsprache (Rédei 1965: 97).

Die Subdialekte Nizjam und Keuši des Mittel-Ob Dialektes ${ }^{24}$ werden in anderen Quellen zur Südgruppe gezählt (verglichen Hajdú/Domokos (Hg.) 1987), repräsentieren aber in jedem Fall Übergangsdialekte zur Nordgruppe (Hajdú/Domokos (Hg.) 1987: 60f.).

Die Nordgruppe verfügt über das kleinste Kasussystem, der Šerkaly-Subdialekt des Mittel-Ob-Dialektes zum Beispiel verfügt über drei Kasus: Nominativ, Lokativ, DativLativ (bei Personalpronomina Nominativ, Akkusativ, Dativ) (Schön 2014: 30). 
Als Südgruppe werden der Irtyš-Dialekt mit seinen Subdialekten Obere und Untere Demjanka, Konda und Krasnojarsk bezeichnet (Honti 1998: 328). Eine genauere Systematisierung der Subdialekte ist heute nicht mehr möglich (verglichen Schön 2014: 28f.), und die Dialektgruppe wird inzwischen als ausgestorben angesehen (Abondolo 1998c: 359; Moseley (Hg.) 2010). Sie wurde entlang des Flusses Irtysch und seiner Nebenflüsse Demjanka und Konda gesprochen (Csepregi 1998/2011: 6).

Die Ostgruppe beginnt an der Mündung des Irtysch in den Ob und verläuft östlich entlang der Nebenflüsse des mittleren Ob. Zur Ostgruppe wird der Surgut-Dialekt um die gleichnamige Stadt (russisch Сургу́т) sowie der Vach-Vasjugan-Dialekt ${ }^{25}$ an den gleichnamigen Flüssen Vach (russisch Bax) und Vasjugan (russisch Васюган) gezählt (Csepregi 1998/2011: 6). Bedeutendere Subdialekte des Surgut Dialektes sind Tremjugan, Jugan und Pim (Schön 2014: 43).

Der Salym-Dialekt entlang des gleichnamigen Flusses (russisch Большо́й Салым) kann als am Übergang zwischen der östlichen und südlichen Dialektgruppe stehend angesehen werden (Honti 1998: 329). Er weist sowohl Charakteristiken der Ostgruppe (morphologisch) als auch der Südgruppe (phonologisch) auf (Csepregi 1998/2011: 6).

Der östlichste Dialekt der Ostgruppe, der Vach-Vasjugan-Dialekt ist der einzige Dialekt des Chantischen, welcher die Vokalharmonie beibehalten hat, und verfügt mit zehn Kasus über das größte Kasussystem im Chantischen, außerdem werden ihm ergativische Züge attestiert (Schön 2014: 23f.). Im Surgut- und Vach-Vasjugan-Dialekt existiert ein paradigmatischer Vokalwechsel in Deklination und Konjugation (Schön 2014: 23f.).

\begin{tabular}{llll}
\hline Nordgruppe & Übergangsbereich & Südgruppe & Ostgruppe \\
\hline Obdorsk & Nizjam & Irtyš & Vach-Vasjugan \\
Kazym & Keuši & - Obere Demjanka & Surgut \\
Berëzovo & & - Untere Demjanka & - Tremjugan \\
Mittel-Ob & & - Konda & - Jugan \\
- Šerkaly & - Krasnojarsk & - Pim \\
Šuryškary & & \\
- Muži & & & \\
- Synja & & \\
Sygva & & & \\
\hline
\end{tabular}

Tabelle 2: Dialektale Gliederung des Chantischen.

25 Hajdú/Domokos (Hg.) 1987 behandeln Vach und Vasjugan als zwei getrennte Dialekte, außerdem vermerken sie dazu noch den Vartovskoe-Dialekt (Hajdú/Domokos (Hg.) 1987: 61). 


\subsubsection{Ungarisch}

Ungarisch ist die uralische Sprache mit den mit Abstand meisten Sprechern, insgesamt rund 14 Millionen (Abondolo 1998a: 428). Das Siedlungsgebiet der Ungarn ist seit dem Ende des neunten Jahrhunderts das Karpatenbecken (Pannonische Tiefebene) im östlichen Mitteleuropa (Szathmáry 1988: 197). Ungarisch ist Amtssprache in Ungarn (ungarisch Magyarorzág) und wird dort von circa zehn Millionen Menschen gesprochen $^{26}$, die restlichen knapp vier Millionen Sprecher verteilen sich größtenteils auf die benachbarten Staaten (Rumänien, Slowakei, Serbien, Kroatien, Slowenien, Ukraine und Österreich) ${ }^{27}$, aber auch in anderen Teilen Europas sowie in Nord- und Südamerika gibt es insgesamt mehrere hunderttausend Sprecher des Ungarischen (Hajdú/ Domokos (Hg.) 1987: 26f.). Im Gegensatz zu den ob-ugrischen Sprachen ist das Ungarische zwar auch dialektal gegliedert, die einzelnen Dialekte unterscheiden sich jedoch relativ wenig voneinander, weswegen eine gegenseitige Verständigung gewährleistet ist (Hajdú/Domokos (Hg.) 1987: 43). Die Unterteilung erfolgt in neun Dialektgruppen, die der Vollständigkeit halber hier aufgelistet werden (Hajdú/Domokos (Hg.) 1987: 44):

- Westdialekte (hierzu gehören unter anderem die in den Komitaten Vas, Zala und Györ-Sopron gesprochenen Dialekte)

- Transdanubische Dialekte (entlang der Donau, in den Komitaten Nord-Somogy, Tolna, Veszprém, Fejér, Komárom)

- Süddialekte (unter anderem gesprochen im Gebiet Szeged-Makó-Hódmezövásárhely)

- Theißdialekte (Gebiet zwischen Donau und Theiß sowie an der Kreisch und Berettyó)

- Nord- oder Paloczen-Dialekte (hierzu gehören unter anderem auch die in der Slowakei gesprochenen ungarischen Dialekte)

- Nordostdialekte (mit den Komitaten Borsod-Abaúj-Zemplén, Szabolcs-Szatmár und nördliches Hajdú-Bihar)

- Mezöség Dialekte (im Gebiet Mezöség in Rumänien)

- Siebenbürger oder Szekler-Dialekte (in Siebenbürgen, Rumänien)

- Csángó-Dialekte ${ }^{28}$

Das Ungarische ist die älteste dokumentierte und verschriftlichte uralische Sprache. Es hat eine reiche Literaturgeschichte, und die Sprachdenkmäler gehen bis ins zehnte Jahrhundert zurück (Hajdú/Domokos (Hg.) 1987: 30). Die Sprachsituation ist demnach eine völlig gegensätzliche zu der der ob-ugrischen Sprachen.

26 Auswärtiges Amt, http://www.auswaertiges-amt.de/DE/Aussenpolitik/Laender/Laenderinfos/01-Nodes_Uebersichtsseiten/Ungarn_node.html (Zugriff vom 14.05.2017).

27 ELDIA-Projekt, http://www.eldia-project.org/ (Zugriff vom 14.05.2017).

28 Z.B. bei Abondolo 1998a: 429. 


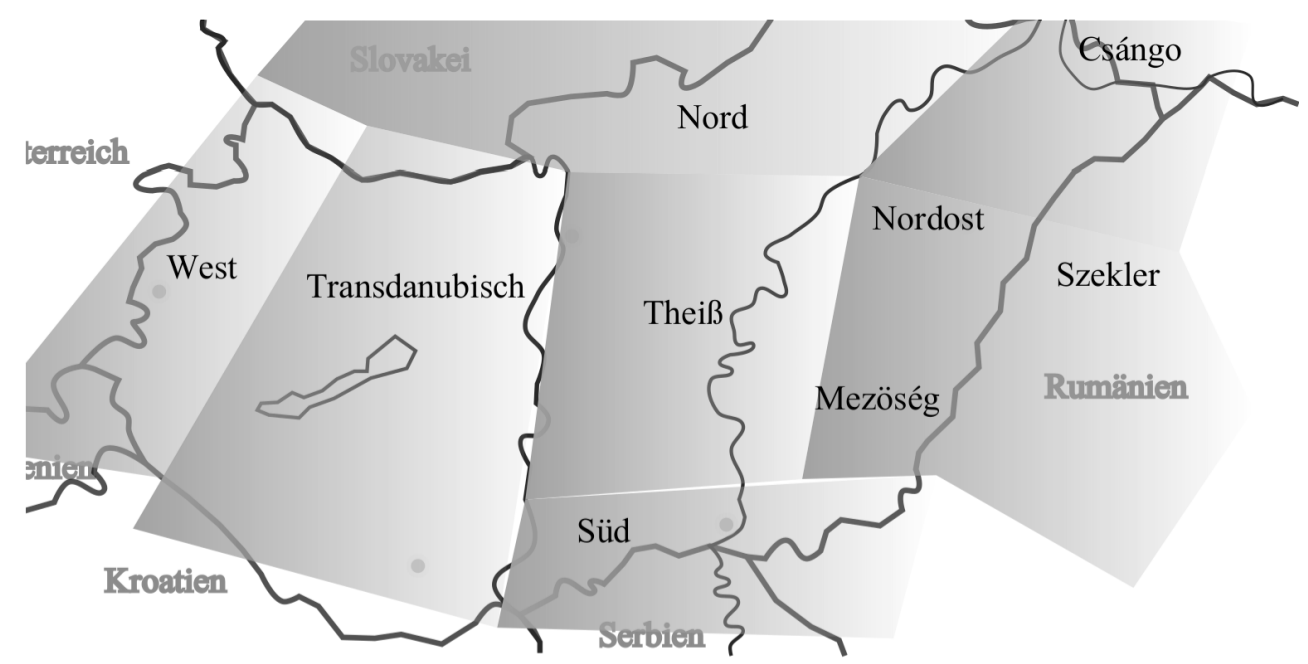

Abbildung 3: Verteilung der ungarischen Dialekte, eigene Adaption nach Hajdú, Péter/Domokos, Péter (Hg.) 1987.

\subsection{Possessivsuffixe}

Der folgende Abschnitt gibt eine Übersicht zum Forschungsgegenstand der vorliegenden Arbeit. Dabei sollen nicht nur Possessivsuffixe selbst und die Konstruktionen, in welchen Possessivsuffixe vorkommen, Thema der Analyse sein. Auch die Terminologie als "Possessivsuffix « und »Possessivkonstruktion « soll diskutiert sowie eine Abgrenzung des Forschungsgegenstands gegenüber den anderen Bereichen der linguistischen Dimension Possession vorgenommen werden.

\subsubsection{Paradigmen der Possessivsuffixe}

Possessivsuffixe sind Personalmarker, welche nominalen Wortarten angefügt werden. Das Paradigma der Possessivsuffixe ist - wie auch die Paradigmen der verbalen Personalmarker - in den meisten Formen aus klitisierten ${ }^{29}$ Personalpronomina entstanden und lassen sich gemeinsam zum Beispiel bis in das Ur-Ob-Ugrische rekonstruieren.

Possessivsuffixe sind aufgrund ihres Ursprungs als Pronomina ebenfalls Pro-Formen, das heißt sprachliche Ausdrücke, welche »sich vor allem auf nominale Bezugselemente beziehen und Vertreterfunktion für diese ausüben, indem sie rückweisend Vorerwähntes aufgreifen « (Bußmann 2002: 538). Wie bei der objektiven Konjugation können mit dem Possessivsuffix zwei Referenten kodiert werden.

29 Aufgrund des vornehmlich agglutinierenden Sprachbautyps des Uralischen wird eine Enklise der Personalpronomina an das vorangehende Verb als Entstehung der verbalen und nominalen Personalmarker angenommen (verglichen z. B. Honti 1998: 341 für die ob-ugrischen Personalmarker). 


\begin{tabular}{|c|c|c|c|c|}
\hline & Personalpronomen & Verbale & suffixe & Nominale \\
\hline & & Subjekt & Objekt & Referenzpunkt \\
\hline $1 \mathrm{SG}$ & *aam, *maa & & ) $\mathrm{m}$ & $*_{-}(\mathrm{V}) \mathrm{m}$ \\
\hline $2 S G$ & $*_{\text {nay, }} *_{\text {ney }}$ & & $\sqrt{n}$ & $*_{-}(\mathrm{V}) \mathrm{n}$ \\
\hline $3 \mathrm{SG}$ & *roy & $*_{-}$ & $*_{-}(\mathrm{V}) \gamma, *_{-}(\mathrm{V})$ & $*_{-}(\mathrm{V}) \gamma,{ }^{*}-\gamma(\mathrm{V})$ \\
\hline $1 \mathrm{DU}$ & $*_{\text {miin }}$ & & iin & $*_{-1 m i i n}$ \\
\hline $2 \mathrm{DU}$ & $*_{\text {niin }}$ & & $*_{\text {-tən }}$ & $*_{\text {-niin, }} *_{\text {-tən }}$ \\
\hline 3DU & *riin & $*^{-}-\gamma$ & $*_{\text {-өiin }}$ & $*_{\text {-riin }}$ \\
\hline 1PL & *maa1, *maan & & ) $\gamma^{w}$ & ${ }^{*}-(\mathrm{V}) \mathrm{\gamma}^{\mathrm{w}}$ \\
\hline $2 \mathrm{PL}$ & *naa1, * naan & & $\left(V_{11}\right)^{j}$ & $*_{-}(\mathrm{V}) \mathrm{n}\left(\mathrm{V}_{11}\right)$ \\
\hline 3PL & $* \gamma V V_{1}, \gamma V_{11}$ & $*_{-}(\mathrm{V}) \mathrm{t}$ & $*_{-}(\mathrm{V}) r(\mathrm{Vnn})$ & $*_{-}(\mathrm{V}) \gamma(\mathrm{Vn})$ \\
\hline
\end{tabular}

Abbildung 4: Rekonstruierte Formen der Personalpronomen und Personalsuffixe für das Ur-Ob-Ugrische, eigene Adaption in IPA nach Honti 1998: 234.

Die Kodierung ist in beiden Paradigmen asymmetrisch, da beide Referenten nicht im selben Umfang kodiert werden: Der aufgrund der Beschreibungsgrundlage als Possessor bezeichnete Referent wird in Person und Numerus kodiert und der als Possessor bezeichnete Referent nur im Numerus:

\begin{tabular}{|c|c|c|}
\hline Referent A & Referent B & Suffix \\
\hline Possessor & Possessum & \\
\hline Person+Numerus & Numerus & \\
\hline$\checkmark$ & . & . \\
\hline$N$ & N & $-P x$ \\
\hline$a m$ & nia:wram & -jaym \\
\hline $1 S G$ & child & $\mathrm{DU}<1 \mathrm{SG}$ \\
\hline \multicolumn{3}{|c|}{,Meine beiden Kinder $\left(\mathrm{NM}, 2145^{30}\right)$} \\
\hline
\end{tabular}

Tabelle 3: Schematische Darstellung der in der Possessivkonstruktion kodierten Referenten.

In manchen Konstruktionsarten entfällt jedoch die Kodierung des zweiten Referenten, siehe dazu Kapitel 5.1.2 und 5.1.3. Im Ob-Ugrischen gibt es drei Numeri, Singular, Dual und Plural, wodurch sich für die Possessivsuffixe ein Paradigma von drei mal drei mal drei Formen für jeweils drei Numeri des Possessum, drei Numeri des Possessors, unterteilt in jeweils drei Personen ergibt. Für das Ungarische, welches keinen Dual besitzt, sind es entsprechend weniger Formen, jeweils zwei Numeri für das Possessum und zwei Numeri in jeweils drei Personen für den Possessor.

30 Wird nur der Beleg und nicht der gesamte Satz als Beispiel angegeben, wird auf ihn mit der zugehörigen fortlaufenden Nummer im Korpus verwiesen 
Im Folgenden werden die Paradigmen der Possessivsuffixe für die in der vorliegenden Arbeit behandelten Dialekte dargestellt. Suffixe, welche nicht im Korpus belegt sind, sind schwach hervorgehoben. Die Formen hier repräsentieren eine Vereinheitlichung der Darstellungen in den zahlreichen Chrestomathien, Grammatiken und Handbüchern in einer IPA-Transliterierung für die ob-ugrischen Dialekte. Das Paradigma des Ungarischen ist vervollständigt mit einer Zusammenfassung von Forgács 2007 und Kenesei/Vago/Fenyvesi (Hg.) 1998 in der ungarischen Orthografie.

\begin{tabular}{|c|c|c|c|c|}
\hline \multirow[t]{2}{*}{ Possessor } & & \multicolumn{3}{|c|}{ Possessum } \\
\hline & & Singular & Dual & Plural \\
\hline \multirow{3}{*}{ SG } & 1. & $-B V m$ & -(j)BVyəm & -(j)anəm \\
\hline & 2. & $-B V n$ & -ayən & -(j)an[ən] \\
\hline & 3. & $-(t) e$ & -(j)aye & -(j)ane \\
\hline \multirow{3}{*}{ DU } & 1. & $-m e n$ & -yamen & -anamen \\
\hline & 2. & $-(e) n$ & -(j)ayen & -nen \\
\hline & 3. & $-(t) e n$ & -ayen & -(j)anen \\
\hline \multirow{3}{*}{ PL } & 1. & -(j)uw & -ayuw & -anuw \\
\hline & 2. & $-(e) n$ & -(j)ayen & -anən \\
\hline & 3. & -(j)anəl & -(j)ayanal & $-a n[a n] ə / /^{31}$ \\
\hline
\end{tabular}

Tabelle 4: Paradigma der Possessivsuffixe des Nord-Mansischen.

\begin{tabular}{|c|c|c|c|c|}
\hline \multirow[t]{2}{*}{ Possessor } & & \multicolumn{3}{|c|}{ Possessum } \\
\hline & & Singular & Dual & Plural \\
\hline \multirow{3}{*}{ SG } & 1. & $-B V m$ & -nəłam & $-t a m$ \\
\hline & 2. & $-B V n$ & -(j)nəłan & $-\tan$ \\
\hline & 3. & $-B V A$ & $-\eta \partial t$ & $-t a t$ \\
\hline \multirow{3}{*}{ DU } & 1. & $-\varepsilon m ə n$ & -(j)nəłmən & -łəmən \\
\hline & 2. & -ən & -(j) நəなən & $-\Varangle ə n$ \\
\hline & 3. & -ən & -(j)nəłən & $-\Varangle ə n$ \\
\hline \multirow{3}{*}{ PL } & 1. & $-B \vee \beta$ & 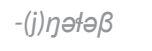 & $-t a \beta$ \\
\hline & 2. & $-B V n$ & -(j)nəłən & $-\tan$ \\
\hline & 3. & $-B V 4$ & - nətat & $-t a t$ \\
\hline
\end{tabular}

Tabelle 5: Paradigma der Possessivsuffixe des Nord-Chantischen (am Beispiel des Kazym-Dialektes).

31 Im Korpus ist nur die Kurzform - anal belegt, die Form -ananal hingegen nicht. 


\begin{tabular}{|c|c|c|c|c|}
\hline \multirow[t]{2}{*}{ Possessor } & & \multicolumn{3}{|c|}{ Possessum } \\
\hline & & Singular & Dual & Plural \\
\hline \multirow{3}{*}{ SG } & 1. & $-B V m$ & -yəłem & -tem \\
\hline & 2. & $-(\partial) n$ & - yәte & $-4 e$ \\
\hline & 3. & $-B V q$ & $-y \partial t$ & $-t e d$ \\
\hline \multirow{3}{*}{ DU } & 1. & -imən & -уәłәmәn & -łəmən \\
\hline & 2. & -in & -yəłən & -tən \\
\hline & 3. & $-(j i) n$ & -yəłən & -łən \\
\hline \multirow{3}{*}{$\mathrm{PL}$} & 1. & $-B \vee \beta$ & $-\gamma \partial \Varangle \partial \beta$ & $-\triangleleft ə \beta$ \\
\hline & 2. & -in & -yəłən & -łən \\
\hline & 3. & $-B V 4$ & $-\gamma \partial t$ & - ted \\
\hline
\end{tabular}

Tabelle 6: Paradigma der Possessivsuffixe des Ost-Chantischen (am Beispiel des Surgut-Dialektes).

\begin{tabular}{llll}
\hline Possessor & & \multicolumn{2}{l}{ Possessum } \\
\cline { 3 - 4 } & & Singular & Plural \\
\hline \multirow{3}{*}{ SG } & 1. & $-B V m$ & $-B V i m$ \\
& 2. & $-B V d$ & $-B V i d$ \\
& 3. & $-(j) a /-(j) e$ & $-B V i /-a /-e$ \\
\hline \multirow{2}{*}{$P L$} & 1. & $-B V n k$ & $-(j) B V i n k$ \\
& 2. & $-B V t o k$ & $-(j) B V i t o k$ \\
& 3. & $-(j) B V k /-(j) a /-(j) e$ & $-(j) B V i k /-(j) e i /-a i$ \\
\hline
\end{tabular}

Tabelle 7: Paradigma der Possessivsuffixe des Ungarischen.

Zu den einzelnen Paradigmen ist Folgendes anzumerken:

- Durch die Suffigierung mit Possessivsuffixen können sich Stammveränderungen ergeben, je nachdem, welchem Stamm sie angefügt werden. Dabei kann es sich um phonologische Wechsel am Stammauslaut handeln, wie etwa dem Wegfall des reduzierten Vokals /ə/ (Schwa) in der letzten Silbe, zum Beispiel Mansisch u:xəl, Schlitten', u:xl-en ,Schlitten'-SG<2SG (Rédei, 1965: 45 ${ }^{32}$ ). Bei manchen Substantiven erfolgt aber auch eine Veränderung im Stamm, zum Beispiel Mansisch xum ,Mann, xumi-m ,Mann'-1SG<SG (Riese, 2001: 20) oder Ungarisch terem ,Raum' term-e ,Raum'-SG<3SG. 
Auch ein Wechsel vom Langvokal zum Kurzvokal kann im Ungarischen erfolgen, zum Beispiel név ,Name‘ nev-em ,Name'-SG<1SG, oder kombiniert, zum Beispiel ló ,Pferd'lov- $a$,Pferd'-SG<3SG. Oftmals wird zwischen Stamm und Suffix ein Bindeglied (Vokale oder - $j$-, in den dritten Personen Einbesitz im Nord-Mansischen auch ein - $t$-) eingefügt. Im Ungarischen sind es aufgrund der Vokalharmonie bis zu vier verschiedene Bindevokale, die auftreten können. Unter Umständen kann auch im Suffix selber ein Schwa erscheinen oder ausfallen. Alle diese Vorkommen werden als Allomorphe des jeweiligen Suffixes betrachtet. In der vorliegenden Arbeit wird lediglich auf das Vorkommen von Allomorphen hingewiesen, nicht jedoch ihre phonetische Umgebung und Realisierung beschrieben, auch Stammveränderungen oder die Etymologie der einzelnen Suffixe sind nicht im Fokus der vorliegenden Arbeit. Detaillierte Beschreibungen hierzu siehe zum Beispiel Honti 1983, Honti 1998 oder Riese 1987. Die Reihenfolge von Kasussuffixen und Possessivsuffixen ist in allen ugrischen Sprachen Px-Cx, wobei die Reihenfolge bei manchen Postpositionen variieren kann (Rédei 1965: 47).

- Die Numerusmarker zur Bildung der Mehrbesitzformen sind in allen drei ugrischen Sprachen andere als die der absoluten Deklination.

- In allen ob-ugrischen Dialekten kann innerhalb des Paradigmas Synkretismus auftreten, das heißt, phonetisch identische Formen kodieren verschiedene Relationen; dieser Zusammenfall ist insbesondere in den Formen der zweiten und dritten Personen Dual- und Mehrbesitz zu finden. Es wurde versucht, in den Glossierungen so eindeutig wie möglich zu bestimmen, um welche Person es sich handelt, dazu wurde auch die Textumgebung mit hinzugezogen. Dennoch ist eine Falschglossierung nicht ganz auszuschließen.

- Im Ungarischen weicht die Verwendung der Possessivsuffixe der dritten Person Singular und Plural im Ein- und Mehrbesitz je nach Konstruktionsart voneinander ab. Das Possessivsuffix der dritten Person Plural Einbesitz - Uk erscheint nur, wenn der Possessor als Leerstelle oder Pronomen in der Konstruktion erscheint:

(10) HU_text_009_0264

a hát -uk -ra

the back $-S G<3 P L \quad-S U B$

'Auf ihre Rücken.'

Erscheint er als Substantiv, wird das Possessivsuffix der dritten Person Singular Einbesitz - $A$ verwendet (Kenesei/Vago/Fenyvesi (Hg.) 1998: 217f.):

(11) $\mathrm{HU} 0243$

$\begin{array}{llllll}a & \text { diplomás } & -o k & \text { szám } & -a ́ & -t \\ \text { the } & \text { graduate } & -\mathrm{PL} & \text { number } & -\mathrm{SG}<3 \mathrm{SG}[\mathrm{PL}] & -\mathrm{ACC}\end{array}$

'Die Zahl der Absolventen.'

Dasselbe gilt für den Mehrbesitz; das Possessivsuffix der dritten Person Plural Mehrbesitz - Aik erscheint nur, wenn der Possessor als Leerstelle oder Pronomen in der Konstruktion erscheint. Erscheint er als Substantiv, wird das Possessivsuffix der dritten Person Singular Mehrbesitz - $A i$ verwendet. Des Weiteren erfordern Numeralia im Ungarischen keine Pluralmarkierung am quantifizierten Kopf. Daher erscheint auch in diesem Fall das Possessivsuffix der dritten Person Singular Einbesitz, obwohl es sich eigentlich um Mehrbesitz handelt: 
(12) HU 550

tiz gazdá -ja

ten landlord $-\mathrm{SG}[\mathrm{PL}]<3 \mathrm{SG}$

'Zehn Herren.'

Im Korpus werden diese Formen gemäß ihrer eigentlichen Kodierung glossiert, die Abweichung wird jedoch mit angegeben.

Bei der Markierung von Mehrbesitz unterscheidet das Ungarische außerdem nicht zwischen mehreren Possessoren und jeweils einem Possessum und mehreren Possessoren mit mehreren Possessum. Im Korpus werden diese Formen ebenfalls gemäß ihrer eigentlichen Kodierung glossiert, die Abweichung wird jedoch mit angegeben.

- Die Formen für zweite Person Singular Mehrbesitz und dritte Person Plural Mehrbesitz im Nord-Mansischen kommen sowohl in einer Kurz- als auch in einer Langform vor; bei ersterer handelt es sich dabei lediglich um den Numerusmarker, das Personalsuffix fällt aus (-an[an]), während in der dritten Person tatsächlich eine gekürzte Form auftritt; beide Kürzungen sind mit eckigen Klammern markiert.

- Im Korpus tritt das Possessivsuffix der dritten Person Plural Mehrbesitz im Mansischen ausschließlich in der Kurzform auf (zum Beispiel -anal), die Langform ist daher ebenfalls im Paradigma kursiv und schwach hervorgehoben. Die Belege im Korpus können dabei allesamt als Mehrbesitz, wobei jedem Possessor jeweils genau ein Possessum zuzuordnen ist, interpretiert werden.

NM_text_010_0048:1

ta:n jowt -anl xassəm -as -ət

$3 P L$ bow $-S G[P L]<3 P L \quad$ start pulling $-P S T \quad-3 P L$

'Sie spannten ihre Bögen.' (das heißt jeder seinen Bogen)

Eventuell handelt es sich hier statt um eine Kurzform des Possessivsuffixes der dritten Person Plural Mehrbesitz um die reguläre Form der dritten Person Plural Einbesitz.

Da das Ungarische bei Mehrbesitz sowie mehreren Besitzern, wie zuvor erwähnt, ebenfalls Unregelmäßigkeiten in der Verwendung des Paradigmas aufzeigt, erscheint die Annahme dieser Verwendungsmöglichkeit auch für das Mansische nicht ganz abwegig. Möglicherweise ist hiermit auch die Kurzform des Possessivsuffixes der dritten Person Plural Mehrbesitz erklärt, sodass das - $n$ - im Suffix nicht für den Mehrbesitz steht und ein der Teil des Personenmarkers entfällt, sondern es sich lediglich um die Form der dritten Person Plural Einbesitz handelt:

\begin{tabular}{lll}
\hline Form & Numerus Possessum & Person +Numerus Possessor \\
\hline Dritte Person Plural Einbesitz: & $-\varnothing$ & - anəl \\
\hline Lange Form Dritte Person Plural Mehrbesitz: & $-(a) n$ & - anəl \\
\hline $\begin{array}{l}\text { Segmentierung Kurzform Dritte Person Plural } \\
\text { Mehrbesitz: }\end{array}$ & $-(a) n$ & -anəl \\
\hline $\begin{array}{l}\text { Alternative Segmentierung Dritte Person Plural } \\
\begin{array}{l}\text { Mehrbesitz, pro Possessor jeweils ein Besitz }= \\
\text { Dritte Person Plural Einbesitz: }\end{array}\end{array}$ & $-(a) n$ & - anəl \\
\hline
\end{tabular}

Tabelle 8: Segmentierung der Form -anəl dritte Person Plural Einbesitz und -ananəl dritte Person Plural Mehrbesitz. 
Dies müsste aufgrund der begrenzten Daten des Korpus noch mit gezielter Elizitierung geprüft werden. Im Korpus werden diese Formen gemäß ihrer eigentlichen Kodierung glossiert, die Abweichung wird jedoch mit angegeben.

- Das Possessivsuffix der dritten Person Singular Dualbesitz wird im Ob-Ugrischen mitunter auch in solchen Konstruktionen verwendet, in welchen es sich eigentlich um Einbesitz handelt:

(14) NM_text_009_0001

e:k ${ }^{w}$ a pirris ${ }^{j} \quad a: k^{w} \quad-e: n \quad$-təl $\quad$ o:l $\quad$-e:y

E:k $^{w}$ a piyris ${ }^{j}$ aunt $-S G<3 D U$-INST be $-P R S[3 D U]$

'Es lebte E:k'a Piyris' mit seiner Tante.'

Der syntaktischen Struktur gemäß handelt es sich hier somit um einen Satz mit Subjekt im Singular, adverbialer Ergänzung in der Form eines Komitativs (mit INST markiert) und Prädikat. Dieses weist jedoch auch den Dual auf, sodass weder beim Prädikat noch beim Numerus des Possessivsuffixes die Kongruenz stimmt. Aus den ugrischen Sprachen ist bekannt, dass die Verwendung des Duals - seltener des Plurals - die Paarigkeit von Referenten hervorhebt und nicht zwangsläufig den Numerus anzeigt:

(15) SK_text_005_0002

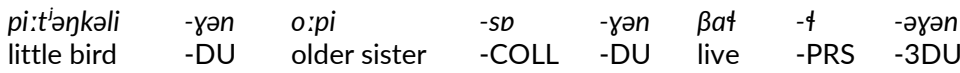

'There lived a little bird and his sister.'

Hier dient der Dual zur Markierung, dass es sich insgesamt um zwei Referenten handelt und nicht jeweils um zwei Pi:tjaykali und um zwei Schwestern, was auch der Dualmarkierung am Prädikat entspricht. Dasselbe kann man für Beispiel (14) annehmen, sodass es sich nicht um Dualbesitz, sondern um insgesamt zwei Referenten handelt, womit auch die Numerus-Kongruenz am Verb wieder stimmig ist. Im Ungarischen, welches zwar kein Dual aufweist, wird ebenfalls oft auf eine Person, welche eine Handlung im Kollektiv ausübt, mit dem Plural verwiesen, sodass auch hier die adverbiale Ergänzung mit als syntaktische Kernrolle am Verb kodiert wird:

(16) HU_text_005_0030

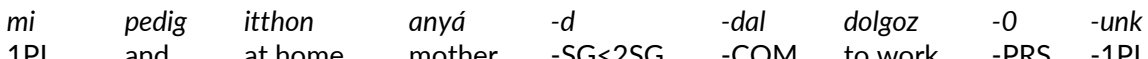

-COM to work -PRS -1PL

'Wir beide, ich und deine Mutter, wir arbeiten daheim.'

Dies scheint auch im Fall der Possessivsuffigierung zu erfolgen, wenn diese eine adverbiale Ergänzung des Subjekts ausdrückt, womit die Dualmarkierungen in Beispiel (16) schlüssig ist.

- Einige Kasussuffixe treten nicht in der possessiven Deklination auf, zum Beispiel der Translativ auf -әу im Sosva-Dialekt des Nord-Mansischen (Murphy 1968: 41), oder haben eine gesonderte Form in der possessiven Deklination, zum Beispiel beim Instrumental im Sosva-Dialekt (in der absoluten Deklination auf $-l$, in der possessiven auf -tal) (Murphy 1968: 39).

\subsubsection{Konstruktionen mit Possessivsuffixen}

Abgesehen von ihrer Etymologie können Possessivsuffixe im Prinzip nur innerhalb einer Konstruktion, das heißt zusammen mit dem Stamm, dem sie angefügt werden, beschrie- 
ben werden, da allein schon die Realisierung der Allomorphe - mit oder ohne Bindeglied - vom jeweiligen Stamm abhängt. Hinzu kommt, dass der im Possessivsuffix kodierte Referent zusätzlich in der Konstruktion realisiert werden kann. Bei den Possessivsuffixen liegt es daher nahe, dann von Possessivkonstruktionen zu sprechen. Hierbei ist jedoch, ebenso wie beim Possessivsuffix selbst, bei der Terminologie einiges zu beachten: Nicht jede Konstruktion mit Possessivsuffix ist zwangsläufig eine »Possessivkonstruktion« und auch nicht in jeder »Possessivkonstruktion « kommt zwangsläufig ein Possessivsuffix vor.

Somit ist es notwendig, kurz auf die Begrifflichkeiten »Possession « (bzw. »Possessivität«) und Possessivkonstruktion einzugehen. »Possession « wird in der vorliegenden Arbeit als eine linguistische Dimension aufgefasst, welche ein abstraktes sprachuniverselles Konzept zum Ausdruck von Relationen zwischen zwei Entitäten bezeichnet (verglichen Aikhenvald/Dixon (Hg.) 2012: 2), analog dazu »possessiv« als Adjektiv (zu den Relationen im Detail, siehe Kapitel 5.1.1.4).

»Possessivkonstruktionen" sind Konstruktionen, welche die in der linguistischen Dimension Possession subsumierten Relationen ausdrücken, und können in drei Gruppen unterteilt werden: attributive, prädikative und externe Possessivkonstruktionen (McGregor (Hg.) 2009: 2). Eine andere Möglichkeit ist die Unterteilung nach dem Kriterium der (Un-) Trennbarkeit (auch Unveräußerlichkeit bzw. sogenannte »alienable« und »inalienable« Possession, Heine 1997: 172f.). In manchen Sprachen basiert die Wahl der Possessivkonstruktion (zum Beispiel verbal vs. attributiv) auf dieser Grundlage, die ugrischen Sprachen machen hier jedoch keinen Unterschied (zum Beispiel Kenesei/Vago/Fenyvesi (Hg.) 1998: 273). ${ }^{33}$

Unter prädikativen Possessivkonstruktionen gruppiert man solche Konstruktionen, welche die Besitzrelation in einer Verbalphrase ausdrücken. Diese kann entweder transitiv sein - das heißt, die Konstruktion besteht aus einem sogenannten Habeo-Verb, der Possessor wird als Subjekt realisiert und das Possessum als direktes Objekt. Die Relation zwischen beiden Entitäten kann in solchen Konstruktionen mithilfe der Semantik des jeweils gewählten Verbs (,haben', ,besitzen', ,beinhalten') konkreter spezifiziert werden als in attributiven Possessivkonstruktionen (Aikhenvald/Dixon (Hg.) 2012: 1).

Intransitive Habeo-Konstruktionen haben ihren Ursprung meist in Lokal- bzw. Existentialsätzen und bestehen aus einem Kopulaverb, welches im Numerus mit dem Possessum kongruiert (Riese 1990: 176f.); es trägt außerdem sämtliche Informationen zu Tempus, Modus und Negation (Kenesei/Vago/Fenyvesi (Hg.) 1998: 157f.). Das Possessum ist Subjekt des Satzes, während der Possessor als sogenanntes Habitivadverbial ${ }^{34}$ fungiert und mit einem Lokalkasus markiert wird. Lokale Habeo-Konstruktionen kommen in den meisten uralischen Sprachen vor, im Ob-Ugrischen wurden sie jedoch größtenteils durch eine transitive Konstruktion ersetzt (verglichen Kangasmaa-Minn 1984: 121).

Die ungarische Habeo-Konstruktion ist ebenfalls eine lokale Habeo-Konstruktion, da für den Dativ $-n A k$, mit dem der Possessor markiert wird, ein lokativer Ursprung

33 Neueste Abhandlungen (Dikken 2015) gehen von einer Auswirkung von (In-)Alienabilität auf die Struktur der attributiven Possessivkonstruktion aus; da die Publikation zum Zeitpunkt der Fertigstellung des Dissertationsmanuskriptes erschienen ist und keine direkten Auswirkungen auf dessen Fragestellung hat, wird sie hier jedoch nicht weiter berücksichtigt.

34 Dieser Begriff ist hauptsächlich in der Ostsee-Fennistik gebräuchlich und beschreibt den Lokalausdruck im Existentialsatz, welcher dem habitiven Satzteil, dem Possessor, in der Habeo-Konstruktion entspricht (verglichen Vilkuna 2008). 
(verglichen Ablativ - $n A ́ l$ ) angenommen wird (Freeze 1992: 590). Die Struktur der Habeo-Konstruktion lässt sich wie folgt darstellen:

\begin{tabular}{llll}
\hline Habitivadverbial & Kopulaverb & Subjekt & Übersetzung \\
\hline a királynak & van & három leánya & 'Der König hatte drei Töchter' (HU, 0788) \\
\hline a fiúnak & nincs & kedve & 'Der Junge hatte keine Lust.' (HU, 0017) \\
\hline a jó szolgál nak & soha sincs & semmi baja & 'Ein guter Diener hat nie ein Problem.' (HU, 0004) \\
\hline an nak & volt & egy fia & 'Welcher einen Sohn hatte.' (HU, 0434) \\
\hline
\end{tabular}

Tabelle 9: Belege für Habeo-Konstruktionen des Ungarischen aus dem Korpus.

Die dritte Gruppe der externen Possessivkonstruktionen wird ebenfalls in der vorliegenden Arbeit nicht explizit berücksichtigt. Als »externe Possession« werden solche Konstruktionen bezeichnet, in welchen die Relation von Possessor und Possessum mithilfe syntaktischer Kernrollen des Satzes ausgedrückt werden, wobei beide jedoch separate Konstituenten bilden, verglichen Payne/Barshi (Hg.) 1999:

We take core instances of external possession (EP) to be constructions in which a semantic possessor-possessum relation is expressed by coding the possessor (PR) as a core grammatical relation of the verb and in a constituent separate from that which contains the possessum (PM). (Payne/Barshi (Hg.) 1999: 3)

Somit kann zum Beispiel auch die Habeo-Konstruktion des Ungarischen als Grammatikalisierung einer Variante der attributiven Possessivkonstruktion mit extrahiertem Possessor zu einem Lokal-/Existentialsatz beschrieben werden (Kenesei et al. 1998: 157). Dementsprechend ist im Hinblick auf die ugrischen Sprachen jedoch noch nicht hinreichend definiert, welche Rolle der externen Possession in der Beschreibung zufallen soll, verglichen Filchenko 2014:

Eastern Khanty has a group of examples whose structural and semantic features could be seen as apparently falling within the external possession phenomenon. Analysis of most examples as external possession can be debated. (Filchenko 2014: 92)

Andere Mechanismen der Derivation, welche proprietäre und damit im weitesten Sinne possessive Relationen ausdrücken können, beispielsweise die denominalen Ableitungssuffixe - $y$ im Mansischen (Скрибник/Афанасева 2009) oder - $w$ im (Ost)-Chantischen (Filchenko 2014: 91), sind ebenfalls nicht Forschungsgegenstand der vorliegenden Arbeit.

\subsubsection{Die attributive Possessivkonstruktion}

In attributiven Possessivkonstruktionen wird das angenommene Besitzverhältnis in einer Nominalphrase ausgedrückt. Sie bestehen aus einem Kopf und einem (oder mehreren) Attributen. Die ugrischen attributiven Possessivkonstruktionen sind head-final, das heißt, die Attribute gehen dem Kopf voraus. Der Possessor wird als Attribut (im Folgenden Modifizierer, siehe dazu auch Kapitel 5.1.1.1 und 5.1.1.2), das Possessum als Kopf der Konstruktion realisiert. Die attributive Possessivkonstruktion ist so gut wie in 
allen uralischen Sprachen belegt und kann in vier Gruppen unterteilt werden, wovon mehrere in einer einzigen Sprache vorkommen können. Die Unterschiede der einzelnen Gruppen belaufen sich auf die morphologische Markierung von Modifizierer und Kopf.

Neben unmarkierten Konstruktionen kann neben dem Kopf auch der Modifizierer markiert werden (zusätzliches dependent-marking):

\begin{tabular}{|c|c|c|c|c|c|}
\hline Typ & Modifizierer & & Kopf & & Beispiele \\
\hline 1.a & Substantiv & & Substantiv & & $\begin{array}{l}\text { háztető (ház-tető) 'Hausdach' HU } \\
\text { ni pot'inka 'der Schuh der Frau' VJ } \\
\text { punk-sow 'Kopfhaut' NM }\end{array}$ \\
\hline 1.b & Substantiv & & Substantiv & $-P x$ & $\begin{array}{l}\text { a ház tete-je 'das Dach des Hauses' HU } \\
\text { rät' oy-ə̂l 'der Kopf des Greises' VJ } \\
\text { Iūpunk-e 'der Kopf des Pferdes' NM }\end{array}$ \\
\hline 1.c & Substantiv & $-C_{x}$ & Substantiv & $-P x$ & a ház-nak a teteje 'das Dach des Hauses' HU \\
\hline 1.d & Substantiv & $-C x$ & Substantiv & & $\begin{array}{l}\text { talo-n katto 'das Dach des Hauses' FI } \\
\text { t'årəs-nə jĕ } \eta k-a \text { 'ins Wasser des Meeres' TJ } 35\end{array}$ \\
\hline
\end{tabular}

Tabelle 10: Typen und Vorkommen der attributiven Possessivkonstruktion in den uralischen Sprachen (Tabelle, Beispiele und Notation nach Honti 2007b: 55f.).

In den ugrischen Sprachen kommen Konstruktionstyp (.a), (1.b) und (1.c) vor. Die Häufigkeit von unmarkierten gegenüber markierten Konstruktionen im Ob-Ugrischen sowie die Frage, ob im Ungarischen Typ (1.a) nur in Komposita zu finden ist, das heißt bei zusammengesetzten Wortformen, muss gesondert analysiert werden, da sich die vorliegende Arbeit auf die Konstruktionstypen, welche mit Possessivsuffixen gebildet werden (Typ (1.b) und (1.c)), und deren verschiedenen Realisierungen konzentriert. Konstruktionen mit Possessivsuffixen sind auch dann zu den Attributivkonstruktionen vom Typ (1.b) zu zählen, wenn der Referent, welcher im Possessivsuffix kodiert ist, als attributiver Modifizierer nicht explizit erwähnt ist, da die Realisierung des Modifizierers unter anderem von pragmatischen Faktoren abhängt. Somit sind folgende Formen der attributiven Possessivkonstruktion der ugrischen Sprachen Bestandteil der Analyse der vorliegenden Arbeit und werden in Kapitel 5.1.1 eingehender beschrieben: Wie eingangs erwähnt ist Possession ein sprachuniverselles Konzept, das heißt, nahezu jede Sprache verfügt über einen oder mehrere der hier vorgestellten Mechanismen, um Possession oder ähnliche Relationen auszudrücken. Welche dieser Relationen, Markierungen, Realisierungen und Faktoren welche Art der Possessivkonstruktion bilden

35 t’åras-nə jĕyk- $a$,Meer'-LOC ,Wasser'-DLAT ist laut Honti eine »ziemlich seltene Konstruktion im Ostjakischen " (Honti 2007a: 56), und nach meiner Auffassung handelt es sich hier nicht um eine Possessivkonstruktion, da (i) der einzige Kasus, welcher in den ugrischen Sprachen dem Modifizierer angefügt werden kann, der Dativ im Ungarischen ist (ii) und hier kein Possessivsuffix vorkommt, aber auch (iii) die Konstruktion nicht als unmarkierter Typ (1.a) angesehen werden kann. Stattdessen ist jedoch zu erwähnen, dass bei Typ (1.d) neben diesem sehr markierten/seltenen Beispiel kein weiteres ugrisches Beispiel gegeben wird, was ebenfalls dafür spricht, dass Typ (1.d) in den ugrischen Sprachen nicht vorkommt. 


\begin{tabular}{|c|c|c|c|c|c|}
\hline Typ & Modifizierer & & Kopf & & Beispiele \\
\hline 1.b.1 & Substantiv & & Substantiv & $-P x$ & $\begin{array}{l}\text { a ház tete-je 'das Dach des Hauses' HU } \\
\text { rät' oy-ôl 'der Kopf des Greises' VJ } \\
\text { lüpunk-e 'der Kopf des Pferdes' NM }{ }^{36}\end{array}$ \\
\hline 1.b.2 & Pronomen & & Substantiv & $-P x$ & $\begin{array}{l}\text { az én mesémre 'zu meinem Märchen' (HU, 0169) } \\
\text { nay xo:ten ßenta 'zu deinem Haus' (KK, 1152) } \\
\text { am piym 'mein Sohn' (NM, 2190) }\end{array}$ \\
\hline 1.b.3 & $\varnothing$ & & Substantiv & $-P x$ & $\begin{array}{l}\text { kezébe 'in meine Hand' (HU, 0439) } \\
\text { ketem 'meine Hand' (SK, 2730) } \\
\text { a:mpe 'sein Hund' (NM, 2227) }\end{array}$ \\
\hline 1.b.4 & Relativsatz & & Substantiv & $-P x$ & $\begin{array}{l}\text { ro:'nxəm ma:tenl 'von dem Ort, an dem er geschrien hatte' } \\
\text { (NM, 2049) }\end{array}$ \\
\hline 1.c.1 & Substantiv & $-C x$ & Substantiv & $-P x$ & a gyereknek a kezében 'in der Hand des Kindes' (HU, 0886) \\
\hline 1.c.2 & Pronomen & $-C x$ & Substantiv & $-P x$ & neki nem gyermeke 'er hatte kein Kind' (HU, 0881) \\
\hline
\end{tabular}

Tabelle 11: Konstruktionstypen der attributiven Possessivkonstruktion in der vorliegenden Arbeit.

und welche Restriktionen sich zwischen möglichen mehreren Konstruktionen in einer Sprache ergeben, ist einzelsprachlich unterschiedlich.

Prädikative Konstruktionen können mittels des jeweiligen gewählten Habeo-Verbs die Relation weitaus mehr konkretisieren als beispielsweise attributive Konstruktionen (Aikhenvald/Dixon (Hg.) 2012: 1), in welchen die Relation der Interpretation durch Sprecher und Hörer bedarf. Auf der anderen Seite sind attributive Konstruktionen dadurch wiederum in einem viel breiteren Kontext anwendbar als die prädikativen Konstruktionen. So herrscht lediglich ein gewisser Konsens im Bereich der sogenannten Kern-Relationen der attributiven Possessivkonstruktion. Dazu gehören (i) Teil-Ganzes-Relationen (hiervon abgeleitet sind auch Lokalangaben wie zum Beispiel ,Spitze/Grund/Anfang/Ende von etwas'), (ii) Verwandtschaftsbeziehungen (sowohl blutsverwandte als auch angeheiratete Verwandtschaft), (iii) konkrete Eigentumsverhältnisse sowie (iv) nicht näher bestimmte Assoziationen jeder Art (Aikhenvald/Dixon (Hg.) 2012: 3f.). Gerade die letzte Gruppe macht deutlich, wie schwer die linguistische Dimension der Possession zu fassen ist. Tatsächlich werden auch nur in einem geringen Anteil der Possessivkonstruktionen konkrete Besitzverhältnisse zum Ausdruck gebracht (Aikhenvald/Dixon (Hg.) 2012: 2). Es wird meines Erachtens deshalb der Konstruktion nicht gerecht, dieser einen Relation eine größere Gewichtung zuzumessen als anderen, begründet diese sich doch lediglich in sozio-kulturellen Faktoren (verglichen Lyons 1983: 327 und Aikhenvald/Dixon (Hg.) 2012: 1). Ebenso ist meines Erachtens die Ableitung sogenannter nicht-possessiver Verwendungen, von denen häufig im Zusammenhang mit dem Possessivsuffix gesprochen wird, nicht zielführend, basiert sie doch einzig und allein auf dem Umstand, dass die sogenannten nicht-possessiven Verwendungen anderer Sprachen nicht dem Konzept der eigenen Sprache bzw. dem primären Forschungsbereich der Possession entspricht (näheres dazu im Kapitel 2). 
Possessivkonstruktionen können somit lediglich als abstrakter Oberbegriff für einen Konstruktionstyp aufgefasst werden, in welchem eine nicht näher bestimmte Relation zwischen zwei Nomen ausgedrückt wird und nicht als formaler Ausdruck von konkreten Besitzverhältnissen. Wenn ein (ausschließlich) semantischer Faktor nicht ausschlaggebend für die Verwendung einer Possessivkonstruktion ist, so muss die Konstruktion selbst die Eigenschaft besitzen, Relationen zwischen zwei Entitäten auszudrücken. Man muss daher eher von einem relationalen Konzept (»relational concept « McGregor (Hg.) 2009: 1) sprechen, welches angewandt wird, will man eine Relation zwischen zwei Entitäten herstellen (und für Substantive, welche inhärent eine Relation zu einer anderen Entität ausdrücken, zum Beispiel Verwandtschaftstermini, wird prototypisch, in manchen Sprachen auch obligatorisch, diese Konstruktion verwendet) (verglichen Janda 2015).

\subsubsection{Weitere Konstruktionstypen}

Synonym zur Bezeichnung "attributive Possessivkonstruktion «, welche in der Benennung den modifizierenden Teil fokussiert, kann der Terminus "adnominale Possessivkonstruktion « angesehen werden, welcher den anderen Bestandteil, den Kopf der Konstruktion, fokussiert. Attributive bzw. adnominale Possessivkonstruktionen - seltener auch als »internal possession construction« bezeichnet (McGregor (Hg.) 2009: 2) sind demnach Nominalphrasen aus einem attributiven Modifizierer und einem Kopf. Der Terminus leitet sich wie bei den Suffixen von einer möglichen Lesart ab und sagt in erster Linie nichts über die formal-funktionalen Eigenschaften der Konstruktion selbst aus. So können auch anderen nominalen Wortarten als Kopf einer Nominalphrase Possessivsuffixe angefügt werden, und es handelt sich dabei nicht um adnominale Possessivkonstruktionen, sondern um eigenständige Konstruktionen mit eigenen Eigenschaften und Funktionen. In den ugrischen Sprachen zählen hierzu drei Gruppen: Nomina, Verbalnomina und Postpositionen. Unter dem Begriff Nomina sind hier Konstruktionen mit Substantiv, Adjektiv, Numerale und Pronomen zusammengefasst.

Definitionen für das Substantiv basieren meist auf formalen, funktionalen und semantischen Kriterien, welche oftmals einzelsprachlich spezifische Charakteristika (zum Beispiel Groß-/Kleinschreibung, Genus) mit einbeziehen. Lässt man diese außen vor, ist eine universelle Definition mitunter zu generell, um wirklich aussagekräftig zu sein. Von den vorhandenen Definitionen zum Substantiv trifft auf das ugrische Substantiv die Deklinierbarkeit zu, das heißt die Aufnahme von Numerus-, Personal- und Kasussuffixen. Dies unterscheidet sie in gewissem Umfang von den Pronomen, welche zum Beispiel bestimmte Kasussuffixe nicht annehmen (zum Beispiel der Komitativ im Ob-Ugrischen) und teilweise über Kasussuffixe verfügen, die das Substantiv wiederum nicht annimmt (zum Beispiel das Akkusativsuffix) bzw. bei bestimmten Kasussuffixe andere, gesonderte Allomorphe aufweisen. Das Substantiv wird im Korpus mit der Abkürzung SuBs gekennzeichnet.

Semantisch lassen sich Substantive unterteilen in »konkrete und abstrakte Substantive: Unter die Konkreta zählen Eigennamen, Gattungsnamen, Sammelbezeichnungen und Stoffbezeichnungen. Abstrakta bezeichnen Eigenschaften, Vorgänge, Beziehungen, Maße« (Bußmann 2002: 664). Diese induktive Unterscheidung ist nur in Hinsicht auf 
die Eigennamen für die vorliegende Arbeit von Berücksichtigung. Eigennamen werden in den Belegen eigens mit der Markierung Nprop gekennzeichnet, unter anderen deshalb, da bei bestimmten Eigennamen, die sich aus mehreren Substantiven zusammensetzen (NM $e k^{w} a p_{i \gamma r i s^{j}}, \mathrm{Ek}^{\mathrm{w}} \mathrm{a}$ Piyris $\left.{ }^{\mathrm{j}}\right)$, oder im Kombination mit Apellativa (NM aki o:jka, Onkel') das Possessivsuffix nur an einem Bestandteil des Kompositums angefügt wird, das mitunter nicht an letzter Stelle der Konstruktion, das heißt headfinal, steht. Diese Formen werden vereinfacht als NProp-Px im Korpus dargestellt. Der zweite Grund, Eigennamen als eigene Gruppe der Substantive zu kennzeichnen, ist der, dass der bisherige Forschungsansatz, der auf der Interpretation von Besitzverhältnissen beruht, bei dieser Verwendung einen Sonderfall suggeriert, da hier die Interpretation eines Besitzverhältnisses nicht ohne Weiteres möglich. Die vorliegende Arbeit will stattdessen die Markierung von Relationen zwischen Personen hervorheben, die nicht nur mit der Verwendung von Verwandtschaftsterminologie, sondern auch mit dem Eigennamen einer Person zum Ausdruck gebracht werden kann.

Syntaktisch können Substantive die Rollen Subjekt, direktes und indirektes Objekt ausüben sowie als Bezugswort für Adpositionen fungieren (Loos (Hg.) 2004b). Hierbei wird das Substantiv deduktiv definiert, da beispielsweise Pronomina dieselben syntaktischen Funktionen ausüben, sich aber im referentiellen Bezug von Substantiven unterscheiden. Während Substantive auf Menschen, Orte, Dinge, Ideen und Konzepte referieren (Loos (Hg.) 2004b), indem sie diese konkret benennen, fungieren Pronomen als Platzhalter (siehe hierzu den folgenden Absatz). Ein weiteres Definitionskriterium für Substantive ist ihre Funktion als Kopf von Nominalphrasen, welche mit Attributen spezifiziert werden können (Bußmann 2002: 664), was der Beschreibung der adnominalen Possessivkonstruktion entspricht, aber als alleiniges Definitionsmerkmal im Hinblick auf die weiteren in der vorliegenden Arbeit vorgestellten Konstruktionstypen mit Possessivsuffix nicht ausreicht.

Der Begriff Nomen (Plural Nomina) wird in der vorliegenden Arbeit als »zusammenfassende Bezeichnung für nominale Wortarten « (Bußmann 2002: 469) verwendet, worunter sowohl Substantive, Adjektive, Pronomina als auch Numeralia fallen (Bußmann 2002: 469). Substantive werden in der vorliegenden Arbeit wie folgt definiert: als nominale Wortart, welche vornehmlich im Satz als Subjekt, direktes und indirektes Objekt fungieren, aber auch weitere syntaktische Rollen bekleiden können (zum Beispiel als Modifizierer). Dabei erstellen sie als "sprachlicher Ausdruck Referenz zu einem Gegenstand der außersprachlichen Realität, auf den sich der Ausdruck bezieht « (Bußmann 2002: 554), und können mit Pro-Formen ersetzt werden.

Pronomina sind eine »nach ihrer Funktion als `Stellvertreter des Nomens einer Nominalphrase bezeichnete Wortart, die eine unter syntaktischem und semantischem Aspekt sehr heterogene Gruppe von Einzeltypen umfasst « (Bußmann 2002: 541). Ihre Gruppierung zu einer Wortart ergibt sich aus der gemeinsamen Eigenschaft des Verweisens. Dabei referieren sie - anders als zum Beispiel Eigennamen - immer abhängig "vom sprachlichen Kontext bzw. der jeweiligen außersprachlichen Situation" (Bußmann 2002: 541) auf unterschiedliche Entitäten (verglichen Bußmann 2002: 541). Insbesondere bei den Personalpronomina ist das Possessivsuffix häufig fossilisiert, das heißt, das Vorkommen der Possessivsuffixe unterliegt keinen pragmatischen oder anderen Bedingungen und die einzelnen Bestandteile lassen sich nicht mehr ohne Weiteres 
segmentieren, da sie wie zum Beispiel im Mansischen fester Bestandteil des Stammes sind oder den Deklinationsstamm des Personalpronomens bilden (Riese 2001: 30f.). In anderen Fällen dienen die Possessivsuffixe als Kasusmarker wie beim Akkusativ im Nord-Mansischen (Murphy 1968: 36), zum Beispiel Personalpronomen erste Person Singular am; Akkusativ: a:nəm, Dativ: a:nəmn.

Andere Pronomina wie Demonstrativpronomina (zum Beispiel Mansisch $t i$, diese/ $\mathrm{r} / \mathrm{s}^{\mathrm{s}}, \mathrm{ta}$,jene $/ \mathrm{r} / \mathrm{s}^{\mathrm{s}}$ ) und Fragepronomen (zum Beispiel Mansisch xotjut ,wer, ,welche,r, $\mathrm{s}^{\mathrm{c}}$ ) können auch mit Possessivsuffixen versehen werden, genauso wie Adjektive oder Numeralia.

Letztere sind eine »in ihrem Status als eigene Wortart umstrittene Klasse von Wörtern" (Bußmann 2002: 477), denen allen die Bezeichnung von "Zahlen, Quantitäten und in Zahlen quantifizierbare Größen- und Mengenangaben « (Bußmann 2002: 477) gemeinsam ist. Da solche Vorkommen jedoch einen relativ geringen Anteil der Belege ausmacht und diese kleine Gruppe dazu noch recht heterogen ist, wird sie in der Analyse der vorliegenden Arbeit nicht berücksichtigt.

Der Begriff "Possessivkonstruktion" bezieht sich demnach in der vorliegenden Arbeit ausschließlich auf attributive Nominalphrasen, deren Kopf ein Substantiv mit Possessivsuffix bildet. Auf diese wird im Weiteren vom syntaktischen Gesichtspunkt aus als "attributive Nominalphrase«, auf den Possessor als »Modifizierer" und auf das Possessum als »Kopf der Nominalphrase« Bezug genommen.

Der Begriff »Verbalnomen« ist in der vorliegenden Arbeit als Oberbegriff für infinite Verbalformen, zum Beispiel Partizipien oder Infinitive, zu verstehen. Die Definition von infiniten Verbalformen ist ebenfalls nicht eindeutig. Laut Bußmann sind sie unkonjugierte Verbformen, »das heißt hinsichtlich Person, Numerus, Tempus, Modus und Genus Verbi nicht gekennzeichnete Verbformen, die Affinitäten zum nominalen [...] Bereich zeigen " (Bußmann 2002: 304). Das Glossary of Linguistic Terms definiert sie als Verbform, welche nicht vollständig nach allen verbalen Kategorien flektiert wird, beispielsweise Tempus, Aspekt, Numerus und Person (Loos (Hg.) 2004a). Beide Definitionen sind nicht gänzlich zutreffend auf die Verbalnomina der ugrischen Sprachen: Diese können nicht nur mitunter Tempus (zum Beispiel Partizip Präsens und Partizip Perfekt) oder Negation (mit dem Negationspartizip - təy im Surgut-Chantischen, Schön 2014: 162f.) ausdrücken, sondern auch nach Person flektiert werden. Dies ist insbesondere im Hinblick auf die vorliegende Arbeit von Bedeutung, da hier ebenfalls das Paradigma der Possessivsuffixe (der nominalen Personalmarker) zum Einsatz kommt. Somit ist der zweite Aspekt der Definition, also die Affinität zum nominalen Bereich, der ausschlaggebendere für die Verbalnomina der ugrischen Sprachen. Dies zeigt sich zum Beispiel durch die syntaktischen Eigenschaften der Verbalnomina - sie fungieren häufig als Nebensatzentsprechungen und können wie andere Nomina syntaktische Kernrollen des Satzes wie Subjekt oder direktes Objekt einnehmen. Sie können außerdem nach Kasus flektiert werden oder in postpositionalen Konstruktionen auftreten. Neben Partizipien und Infinitiven verfügen die ob-ugrischen Sprachen über zwei weitere infinite Verbalformen: Konverben und das »Nomen Actionis«.

Konstruktionen mit Konverben bilden adverbiale Nebensatzkonstruktionen, sie werden daher zum Beispiel auch als Verbaladverb oder Adverbialpartizip bezeichnet, 
mitunter wird auch der Begriff »Gerundium« synonym zum Konverb verwendet. Die vorliegende Arbeit benutzt den aus der Altaistik entlehnten Begriff Konverb in der Definition des OUL-Projektes. ${ }^{37}$ Das Nomen Actionis ist eine infinite Verbalform, welche Handlungen und Vorgänge ausdrückt. Sie wird auch als Verbalabstraktum oder Verbalsubstantiv bezeichnet (Bußmann 2002: 469), die Übersetzung der englischen Bezeichnung "verbal noun« (Loos (Hg.) 2004d) als "Verbalnomen« wird in der vorliegenden Arbeit nicht verwendet, um keine Verwechslungen mit Verbalnomina hervorzurufen; "Verbalnomen " (Plural Verbalnomina) ist in der vorliegenden Arbeit der Oberbegriff für sämtliche infinite Verbalformen der ugrischen Sprachen, wohingegen "Nomen Actionis« (auch Verbalsubstantiv) eine bestimmte im ugrischen vorkommende infinite Verbalform in der Definition des OUL-Projektes ${ }^{38}$ bezeichnet. Der Begriff »infinite Verbalform« wird aus oben genannten Gründen ebenfalls nicht weiter verwendet.

Postpositionen sind Adpositionen, welche dem Bezugswort folgen und beispielsweise lokal- oder temporal-adverbiale Bestimmungen ausdrücken. Ihren Ursprung haben Postpositionen unter anderem in Substantiven, welche sich zu Synsemantika grammatikalisiert haben (Schön 2014: 80), und können somit im weitesten Sinne als nominale Wortart angesehen werden. Auch Postpositionen können mit Possessivsuffixen versehen werden.

Die in der vorliegenden Arbeit analysierten Konstruktionen mit Possessivsuffixen sind demnach die folgenden:

\begin{tabular}{|c|c|c|c|c|c|}
\hline Typ & Modifizierer & Kopf & & Abkürzung & Beispiele \\
\hline 1 & $\begin{array}{l}\text { Relativsatz/ } \\
\text { Substantiv/ } \\
\text { Pronomen/ø }\end{array}$ & Substantiv & $-P x$ & SUBS-Px & $\begin{array}{l}\text { a ház tete-je 'das Dach des Hauses' } \\
\text { (HU, siehe Tabelle 10) }\end{array}$ \\
\hline 2 & $\begin{array}{l}\text { Substantiv/ } \\
\text { Pronomen/ø }\end{array}$ & Verbalnomen & $-P x$ & VN-Px & $\begin{array}{l}\text { menk i:ki jaqə łanmeł łe:tnə 'Als der Menk-Alte } \\
\text { eingetreten war' (SK_text_001_0017:1) }\end{array}$ \\
\hline 3 & $\begin{array}{l}\text { Substantiv/ } \\
\text { Pronomen/ø }\end{array}$ & Postposition & $-P x$ & PostP-Px & nan xuripan 'wie du' (NM 1703) \\
\hline
\end{tabular}

Tabelle 12: Konstruktionen mit Possessivsuffix in der vorliegenden Arbeit.

Die Konstruktion mit Substantiv als Kopf steht dabei im Fokus der vorliegenden Arbeit. Auf Postpositionen und Verbalnomina wird in Relation darauf eingegangen, das heißt, die Analyse dieser Konstruktionstypen erfolgt im Vergleich bzw. im Kontrast zur Konstruktion Subs-Px und erhebt nicht den Anspruch, die Konstruktionen beider Wortarten vollständig zu beschreiben.

Wie die Beschreibung des Forschungsgegenstandes dargelegt hat, tragen weder Possessivkonstruktionen noch Possessivsuffixe die inhärente Bedeutung "possessiv«

37 Ob-Ugric Database, Abschnitt eGrammar, Stichpunkt »Converb«, http://www.oudb.gwi.uni-muenchen. de/index.php?abfrage=comments\&subnavi=subgrammar (Zugriff vom 14.05.2017).

38 Ob-Ugric Database, Abschnitt eGrammar, Stichpunkt Nomen Actionis«, http://www.oudb.gwi.unimuenchen.de/index.php?abfrage=comments\&subnavi=subgrammar (Zugriff vom 14.05.2017). 
in sich, sodass beide Termini die zugrunde liegenden Eigenschaften nicht adäquat repräsentieren. Possessivsuffixe sind vielmehr morphologische Marker, welche Referenz erstellen auf die Personen, welche sie kodieren, und mitunter in Konstruktionen, welche Relationen zwischen zwei Entitäten ausdrücken, vorkommen können. Diese und weitere Verwendungen sind Gegenstand der Analyse in der vorliegenden Arbeit. 


\section{Forschungsstand}

Die Beschreibung - und damit auch die terminologische Bezeichnung der Possessivsuffixe - nimmt ihren Ursprung im Bereich der Dimension Possession und deren sprachlicher Realisierung. Um den Stand der Forschung zu uralischen respektive ugrischen Possessivsuffixen zu skizzieren, muss daher der Forschungsstand zur Possession mit einbezogen werden. Da die Forschung zur Possession in der Uralistik dabei maßgeblich ihre Anfänge in vergleichenden bzw. adaptierten Abhandlungen zum Forschungsthema in den indo-europäischen und anderen Sprachfamilien hat, muss auch die allgemeine Forschung mit berücksichtigt werden, um die bisherige Beschreibung des Possessivsuffixes einordnen zu können. Zu Beginn dieses Kapitels wird daher ein kurzer Einblick in die Forschungsgeschichte zur Possession in der Linguistik gegeben, um danach diejenigen Forschungsrichtungen eingehender zu betrachten, welche Relevanz für die Erforschung der Possessivsuffixe im Uralischen und insbesondere in den ugrischen Sprachen hat. ${ }^{39}$ Das Kapitel ist hauptsächlich chronologisch aufgebaut, die aktuellsten Forschungen, welche zum Teil mit in die vorliegende Arbeit eingeflossen sind, befinden sich am Ende des Kapitels. Größtenteils entspricht die vorwiegend chronologische Sortierung den einzelnen Forschungsschwerpunkten, beginnend mit der allgemeineren, onomasiologisch basierten Forschung (von der Funktion des Markers in Possessivkonstruktionen zur Form Possessivsuffix) hin zu einzelnen Konstruktionsarten und zuletzt zum Possessivsuffix selbst, im Kontext verschiedener teilweise aufeinander aufbauender oder ineinander übergehender Forschungsrichtungen (von Theorien zur Definitheit zu kognitiv-pragmatischen Ansätzen).

Die Beschreibungen zur Dimension Possession reichen bis in den Anfang des letzten Jahrhunderts zurück, wenngleich anfangs eher in einem linguistisch-philosophischen Ansatz, zum Beispiel bei Cassirer [1926] 1977. Eine der ersten Abhandlungen zur Dimension Possession, welche außerdem zu erwähnen ist, ist die des Philosophen und Ethnologen Lucien Lévy-Bruhl 1914, da diese sich der Dimension Possession in einer austronesischen Sprache (Bußmann 2002: 109) widmet, das heißt, die linguistische Beschreibung von Possession hat ihren Ursprung nicht wie vielleicht zu erwarten im Kontext der indo-europäischen Sprachen, wenn auch die ersten Werke für diese Sprachfamilie nicht lange auf sich warten ließen, zum Beispiel Bally 1926 (Stolz 2008: 3f.). Die tatsächliche systematische Forschung zur Possession in der Linguistik beginnt in den 1970er- und 1980er-Jahren mit Werken von Seiler (Seiler 1973; Seiler 1983a; Seiler 1983b) und Ultan 1978 bietet bis heute Grundlage für sämtliche Forschungsrichtungen zur Dimension Possession (Stolz 2008: 4). Die Forschungsrichtungen sind dabei ebenso vielfältig wie die in den Possessivkonstruktionen ausgedrückten Arten von Relationen, die formale Markierung und die syntaktische

39 Es wurde versucht, einen möglichst umfassenden Forschungsüberblick zu geben und dabei alle relevanten Forschungen zum Thema zu berücksichtigen. Die russische Forschungsliteratur fand dabei jedoch keine nennenswerte Berücksichtigung; die Aussagen können daher nicht uneingeschränkt auf diese abgeleitet werden. Der Umfang der behandelten Forschungen kann jedoch als Kompensation für die nicht herangezogenen russischen Quellen bestehen. 
Realisierung der Referenten, und ist zudem einzelsprachlich unterschiedlich, sodass sich jede Forschungsrichtung je nach Sprachtyp/Sprachfamilie oder Konstruktionstyp einem anderen Aspekt widmet. Damit kann der Forschungsstand zur Possession in der Linguistik nur grob mit den wichtigsten Vertretern und Werken nachgezeichnet werden. Erwähnenswert ist hierbei jedoch, dass dabei bislang das Gesamtkonzept Possession in den vorwiegend deskriptiven Grammatiken keinen einheitlichen Beschreibungsstatus erfahren hat - statt eines eigenen Kapitels zur Possession finden sich des Öfteren die relevanten Informationen zum Ausdruck von Possession in der jeweiligen Sprache eingestreut in damit in Verbindung stehenden Abschnitten, beispielsweise in den indo-europäischen Sprachen bei P(ossessivp)ronomina oder Genitivkonstruktionen (Stolz 2008: 5).

\subsection{Von der Funktion zur Form - die onomasiologisch-basierte Forschung}

Der vermutlich größte Anteil an linguistischer Forschung im Bereich Possession folgt dem onomasiologischen Ansatz, das heißt, es wird von der Funktion der Konstruktion, eine possessive Relation auszudrücken, deren formale Realisierung in den verschiedenen Sprachen beschrieben. Ein vielfach diskutierter Aspekt ist dabei die Semantik, das heißt die Arten von Relationen, welche in den Konstruktionen ausgedrückt werden können. Erwähnenswerte Publikationen, welche die Possession unter diesem Gesichtspunkt betrachten, sind zum Beispiel Barker 1995, welcher die so ziemlich erste Gesamtbeschreibung der Dimension Possession für das Englische lieferte und Aikhenvald/ Dixon (Hg.) 2012, welches eines der aktuellsten Werke in diesem Bereich darstellt und verschiedene Sprachfamilien berücksichtigt.

Der onomasiologische Ansatz zur Beschreibung von Possession ist derjenige, welcher auch primär in den Handbüchern, Grammatiken und Chrestomathien in der Uralistik herangezogen wurde und sich für einen kurzen Einblick in das Thema im Rahmen einer gesamtsprachlichen Beschreibung prinzipiell anbietet. Im Allgemeinen wird hier unter dem formalen Gesichtspunkt auf die Markierung mittels Possessivsuffixen hingewiesen, der Fokus der Beschreibung liegt jedoch auf der Semantik der Konstruktion. Auf andere Konstruktionsarten, in welchen Possessivsuffixe beteiligt sind, zum Beispiel Verbalnomina oder Postpositionen, wird oft in einem abschließenden Satz verwiesen. Hier werden exemplarisch zwei Handbücher, zwei ungarische Grammatiken sowie jeweils eine Chrestomathie einer ob-ugrischen Sprache vorgestellt.

Im einleitenden Kapitel zum Handbuch »The Uralic Languages« (Abondolo (Hg.) 1998) werden Possessivsuffixe als Personalmarker der nominalen Wortarten geführt, deren am meisten verbreitete Funktion die des Ausdrucks des Possessors ist (Abondolo 1998b: 22). Die Aufsätze zu den obugrischen Sprachen verweisen auf ein nominales Personalparadigma (zum Beispiel Abondolo 1998a: 438), welches am häufigsten den Possessor ausdrückt und schon für das Ur-Ob-Ugrische belegt ist (Honti 1998: 341). Während in der allgemeinen Einleitung das Konzept Possession erwähnt wird, welches durch die Verbindung von Nominalphrasen ausgedrückt wird, wobei der Possessor auf verschiedene Weise markiert wird (Abondolo 1998b: 32), ist in den Beschreibungen zum Ob-Ugrischen die Syntax der Nominalphrase der Ausgangspunkt, von denen eine 
Konstruktionsart als Ausdruck von Possession genutzt wird, zum Beispiel bei Abondolo 1998c zum (Tremjugan) Chantischen:

Noun phrases can be linked in two basic ways. One type of linkage forms possessive constructions. (Abondolo 1998c: 378f.)

In den einzelnen Kapiteln zu den jeweiligen Sprachen, welche einen umfassenden Überblick der wichtigsten linguistischen Kategorien zu jeder Sprache ermöglichen sollen, kann eine detaillierte Beschreibung der Possessivsuffixe und ihrer Verwendung kaum erwartet werden; dennoch muss hier auf kleinere Inkonsistenzen hingewiesen werden. Auf den ersten Blick ist die Beschreibung in allen vier Kapiteln zu den ugrischen Sprachen relativ einheitlich, das heißt es, wird in denselben Themenbereichen - nominale Deklination und Syntax - Bezug auf Possessivsuffixe genommen. Während Abondolo 1998a und 1998c jedoch analog zur Einleitung den Terminus "possessed " gebraucht, bezieht sich Keresztes 1998 mit dem Begriff "possession « auf das Possessum (Keresztes 1998: 418) und spricht von »Possessiver Deklination « (Keresztes 1998: 410), wohingegen Abondolo 1998a und 1998c von der nominalen Kategorie Person sprechen. Im Kapitel zu Ungarisch fügt er den Ausdruck »Possessive Suffixes« mit hinzu (Abondolo 1998a: 438), im Kapitel zu Chantisch hingegen nicht - im Beispielparadigma tauchen die Bezeichnungen »Possessor « und »Possession« auf (Abondolo 1998c: 365 und 366).

Honti 1998 bespricht im Abschnitt Syntax die - im heutigen Ob-Ugrischen kaum noch vorkommende - lokale Habeo-Konstruktion als Possessivkonstruktion, erwähnt die adnominale Konstruktion hingegen nicht (Honti 1998: 350). Problematisch ist meines Erachtens, wie auch unter Berücksichtigung der komprimierten Beschreibung auf die Verwendungsweise des Paradigmas der Possessivsuffixe hingewiesen wird. Dass die Suffigierung mittels Possessivsuffix nicht obligatorisch ist, ist in jedem Kapitel vermerkt, doch nur im Kapitel zum Ungarischen wird auf die Verwendung des gesamten Paradigmas hingewiesen und auch hier nur im Zusammenhang mit der nominalen Personalmarkierung. Ansonsten wird als mögliche Markierung von Person in einer (possessiven) Nominalphrase nur auf das Possessivsuffix dritte Person Singular hingewiesen, teilweise mit einer Formulierung, die meines Erachtens suggeriert, dass die Möglichkeit der Markierung durch andere Possessivsuffixe ausgeschlossen ist, zum Beispiel Keresztes 1998 zum (Pelym) Mansischen (verglichen auch Abondolo 1998c: 379 und Abondolo 1998a: 451):

In possessive constructions the possessor is similarly unmarked. The possession, on the other hand, may be marked with the third person singular suffix. (Keresztes 1998: 418)

Ähnlich ist die Beschreibung zu Possessivsuffixen im Handbuch von Sinor (Hg.) 1988. In den Kapiteln zu den ob-ugrischen Sprachen wird auf Possessivsuffixe im Zusammenhang als morphologische Marker der nominalen Kategorie hingewiesen, sowie im Bereich der Syntax als Bestandteil von Possessivkonstruktionen. Anders als bei Abondolo (Hg.) 1998 wird hier auf den Zusammenhang der Markierung mittels Possessivsuffixen und Personalpronomen hingewiesen, zum Beispiel (Surgut) Chantisch:

Wenn das Possessivattribut ein Personalpronomen ist, welches auch fehlen kann, muss das possessive Verhältnis am Besitzwort durch Possessivsuffix bezeichnet werden [...]; wenn das Possessivattribut ein Nomen ist, kann das Besitzwort mit oder ohne Possessivsuffix stehen. (Honti 1988a: 193) 
Weitere Handbücher der Uralistik sind Lytkin/Majtinskaja (Hg.) 1966, Benkö/Samu (Hg.) 1972, Hajdú/Domokos (Hg.) 1987 oder Csepregi 1998/2011.

In der »Ungarischen Grammatik« von Tamás Forgács (im Folgenden Forgács 2007) sind Possessivsuffixe Thema mehrerer Kapitel und Abschnitte. Die Deklination unterteilt Forgács 2007 in Grund- und Endsuffixe, wobei Possessivsuffixe zu den Grundsuffixen der Substantive (da sie den Kasussuffixen, das heißt Endsuffixen vorangehen) zählen (Forgács 2007: 60f.). Im Zusammenhang mit anderen Wortarten, zum Beispiel Infinitiven, Pronominaladverbien (possessivsuffigierte Postpositionen), wird auch auf die Suffigierung mit Possessivsuffixen hingewiesen; hier werden sie jedoch als Endsuffixe klassifiziert, da keine weiteren Suffixe folgen können (Forgács 2007: 64f.). Die Bildung des Mehrbesitzes erhält ein eigenes Kapitel, kontrastiv zur Pluralbildung in der absoluten Deklination. Ein ausführliches Paradigma der Possessivsuffixe - im Ein- und Mehrbesitz - befindet sich im anschließenden Kapitel »Die paradigmatischen Besitzerzeichen «. Hier wird auch die attributive Possessivkonstruktion behandelt. Unter dem Unterpunkt »Besonderheiten im Gebrauch der Besitzerzeichen« beschreibt Forgács 2007 weitere, »noch kompliziertere« (Forgács 2007: 135) Aspekte im Zusammenhang mit der Possessivsuffigierung. Darunter fallen unter anderem die Vorkommen des Bindegliedes - $j$ - bei Stämmen mit Konsonantauslaut, aber auch die Habeo-Konstruktion (Forgács 2007: 135f.). Die Grammatik von Forgács 2007 ist als Lehrwerk für UngarischLernende Muttersprachler des Deutschen konzipiert; die Beschreibung der Verwendung der Possessivsuffixe ist demnach stark an den Vergleich mit der Possessivkonstruktion des Deutschen angelehnt. Die Struktur der attributiven Possessivkonstruktion wird im Vergleich zur Genitivkonstruktion des Deutschen beschrieben (Forgács 2007: 132), die für das Deutsche verwendeten Typen, welche in der Genitivkonstruktion ausgedrückt werden können, werden auf die in der ungarischen Possessivkonstruktion vorkommenden Relationen abgeleitet (Forgács 2007: 425). Auf den dependent-marker wird somit als Genitivsuffix verwiesen. Beide Konstruktionstypen (mit und ohne dependent-marking) werden zwar als "gleichwertig" (Forgács 2007: 391) beschrieben, dann jedoch eine detaillierte Auflistung gegeben, wann das sogenannte Genitivsuffix obligatorisch ist und wann nicht. Außerdem wird das dependent-marking in der ungarischen Konstruktion im Zusammenhang mit der Satzstellung (Possessor unmittelbar vor dem Possessum bzw. durch einen Artikel getrennt) erläutert, ebenso wird auf das Vorkommen von Personalpronomina in dieser Hinsicht verwiesen.

Im Rahmen der Routledge-Reihe »Descriptive Grammars« ist auch eine Grammatik für das Ungarische erschienen (im Folgenden Kenesei/Vago/Fenyvesi (Hg.) 1998). Hier sind Possessivsuffixe Thema der Beschreibung mehrerer Kapitel, unter anderem zur Syntax, Morphologie und zum Lexikon. Im Kapitel zur Syntax beispielsweise wird wie erwartet auf die verschiedenen Konstruktionstypen zum Ausdruck von Possession im Ungarischen eingegangen. Die Possessivkonstruktion wird in ihren zwei Konstruktionstypen ${ }^{40}$ beschrieben und auf die Terminologie im Hinblick auf die Grammatiktradition wie folgt verwiesen:

40 Typ (1.b) und (1.c) der vorliegenden Arbeit; bei Kenesei/Vago/Fenyvesi (Hg.) 1998 als »compact« und »extended construction« bezeichnet (Kenesei/Vago/Fenyvesi (Hg.) 1998: 94). 
Grammatical tradition regards possessive constructions as rattributives, though not adjectival, and lumps them together with adjectival attributes [...]. However, they have nothing in common with the well-known possessive adjectives of, for example, a number of Slavic languages. [...] the possessor in [...] the scompacts possessive construction, has nominative case and it has a definite article to its left, at least in some dialects [...]. In [...] the rextended c construction, the possessor has dative case and the article is to its right. (Kenesei/Vago/Fenyvesi (Hg.) 1998: 94)

In Hinblick auf die Realisierung des Possessors in der Konstruktion vermerkt Kenesei/ Vago/Fenyvesi (Hg.) 1998, dass bei Koreferenz mit einer anderen Nominalphrase der Possessor meist als Leerstelle realisiert wird und eine Realisierung mit Personalpronomen meist auf fehlende Koreferenz mit anderen Referenten (»disjoint reference « Kenesei/Vago/Fenyvesi (Hg.) 1998: 124) im Satz hinweist. Auf das Possessivsuffix selbst wird nicht weiter eingegangen (Kenesei/Vago/Fenyvesi (Hg.) 1998: 124). Das Kapitel zur Morphologie verweist auf die Bildung der attributiven Possessivkonstruktion (»The possessive relationship is expressed with personal possessive suffixes [...] attached to the thing possessed « Kenesei/Vago/Fenyvesi (Hg.) 1998: 214), im Unterpunkt »Possessed « (Kenesei/Vago/Fenyvesi (Hg.) 1998: 216) wird die Verteilung der Allomorphe dargelegt. Im Kapitel fünf »Lexicon« wird beim semantischen Feld »Kinship terminology« auch auf die Suffigierung mit Possessivsuffixen verwiesen (Kenesei/Vago/Fenyvesi (Hg.) 1998: 456f.).

Die »Northern Ostyak Chrestomathy« von Károly Rédei (im Folgenden Rédei 1965) stellt die Possessivsuffixe anhand des Paradigmas des Muži-Subdialektes des ŠuryškaryDialektes vor. Auf viereinhalb Seiten wird das gesamte Paradigma in allen möglichen Kombinationen von Person und Numerus des Possessors und Numerus des Possessum beispielhaft für zwei Substantive durchdekliniert. Danach folgt eine ausführliche Erläuterung des Systems mit Hinweisen zu Synkretismus, Stammveränderungen (zum Beispiel Wegfall des Schwa in der letzten Silbe), Wahl des Bindevokals (zum Beispiel $S G<1 S G$-em verglichen mit -am bei Verwandtschaftstermini) sowie zur »unregelmäßigen Bildung« (»irregular inflection« Rédei 1965: 46) der possessivsuffigierten Formen von ne: $\sim n e: y$ - ,Frau' und $x u: \sim x u: j-$,Mann', welche meines Erachtens ebenso regelmäßig (verglichen Mansisch xum, Mann, Rédei 1965: 28) wie der paradigmatische Vokalwechsel bei Substantiven mit Auslaut auf - $i$ ist: e:wi ,Mädchen' e:we- $m$,Mädchen'SG $<1 S G$. Abschließend wird die zusätzliche Kasusdeklination thematisiert (Rédei 1965: 44f.). Des Weiteren wird explizit auf die Suffigierung durch Possessivsuffixe bei den Verbalnomen hingewiesen (Rédei 1965: 74), wohingegen sie bei der Auflistung der Postpositionen unerwähnt bleibt, obwohl hier auch Beispiele mit Possessivsuffix aufgelistet sind, zum Beispiel ma: eltem, von mir` (Rédei 1965: 75).

Béla Kálmán beschreibt in seiner »Chrestomathia Vogulica« (im Folgenden Kálmán 1963) die possessive Deklination, wie er die Possessivsuffixe bezeichnet. Zunächst stellt er das Paradigma des singularischen Besitzes vor, jeweils mit einer Form für Stämme mit Vokal- und für Stämme mit Konsonantauslaut, und gibt die Marker für Dual- und Pluralbesitz an; er trennt demnach das Suffix in zwei Bestandteile. Danach geht er auf Stammveränderungen bei der Suffigierung und die Allomorphe des Suffixes selbst ein, als dritte Bemerkung gibt er an, dass auch Postpositionen mit Possessivsuffixen versehen werden können und verweist weiter auf das Ungarische. Zuletzt gibt er die 
Reihenfolge von Kasussuffixen (bei Kálmán 1963 »Adverbialsuffixe«) und Possessivsuffixen an. Abschließend folgt eine weitere, welche das gesamte Paradigma mit sämtlichen Numeri des Possessum und Personen und Numeri des Possessors angibt, jeweils an einem Beispiel mit Vokal- und einem mit Konsonantauslaut (Kálmán 1963: 30f.). Die gesamte Beschreibung der Possessivsuffixe ist sehr kurz, sie erstreckt sich insgesamt über zwei Seiten, wobei hier sowohl eine ungarische als auch eine deutsche Version der Beschreibung geliefert wird, den Großteil der Beschreibung nehmen die Tabellen ein (etwa eine halbe Seite Beschreibung gegenüber eineinhalb Seiten an Paradigmen). Hinweise auf andere Konstruktionsarten, zum Beispiel mit Verbalnomina, fehlen.

Die Beschreibung in Kálmán »Vogul Chrestomathy« (Kálmán 1965) ist im Prinzip eine englische Übersetzung der Beschreibung in seiner ungarischen/deutschen Chrestomathie zwei Jahre zuvor (Kálmán 1963). Auch hier besteht die Abhandlung zu den Possessivsuffixen größtenteils aus (denselben) Paradigmen, und bei weiteren Konstruktionsarten wird nur auf Postpositionen verwiesen.

Die beiden Chrestomathien zu den ob-ugrischen Sprachen sollen exemplarisch für die Beschreibung der Possessivsuffixe in den Chrestomathien bis in die Mitte des 20. Jahrhunderts dienen, sind jedoch nicht die einzigen Werke. Der Vollständigkeit halber sei hier zum Beispiel auf Gulya 1966, Murphy 1968 und Honti 1984 verwiesen. ${ }^{41}$ Neuere Grammatiken, die aktuelle Forschungsströmungen mitberücksichtigen, werden in einem späteren Abschnitt dieses Kapitels thematisiert.

\subsection{Spezifische Abhandlungen zur Possessivkonstruktion}

Spezifische Abhandlungen zur Possessivkonstruktion gibt es für die ugrischen Sprachen kaum, hier sei zum Beispiel der Aufsatz von Szabolcsi 1981 oder Kangasmaa-Minn 1984 erwähnt. Letzterer thematisiert hauptsächlich die prädikative Konstruktion und analysiert die attributive Konstruktion in Relation zu dieser (Kangasmaa-Minn 1984: 122f.). 1985 ist zudem eine Monografie von Anna Biermann (im Folgenden Biermann 1985) mit dem Titel »Possession und Zuschreibung im Ungarischen « in der Reihe »Continuum « erschienen.

Biermann 1985 definiert eingangs den Begriff Possession als Zusammenfassung von »Konstruktionen, deren gemeinsamer Nenner etwa als Signalisierung einer Zugehörigkeitsrelation zwischen zwei Substanzen [...] angegeben werden kann« (Biermann 1985: 1), und thematisiert auch die Problematik des Begriffes:

Hierbei haben wir es mit einer Vielzahl von außersprachlichen Beziehungen zu tun (Besitz [...], Teil-Ganzes-Relationen, Verwandtschaftsbeziehungen, usw.), die durch eine relativ geringe Anzahl von Konstruktionen sprachlich dargestellt werden. Diese Konstruktionen werden traditionellerweise spossessivische Konstruktionen ‘ genannt, obwohl keine von ihnen ausschließlich Possession signalisiert. (Biermann 1985: 2)

41 Diese Liste enthält nicht alle Chrestomathien und grammatikalischen Abrisse zum Ob-Ugrischen, welche bis ins 19. Jahrhundert zurückreichen (z. B. Ahlqvist 1894). 
Biermann 1985 verwendet dazu eine weitere Begrifflichkeit, die »Zuschreibung«, als welche sie folgende Relationen definiert:

Bei der Zuschreibung ist keine Zugehörigkeitsrelation involviert, sondern eine Relation in dem Sinne, dass einem A ein B als charakterisierende Größe zugeschrieben wird, wobei B keine bloße Eigenschaft ist. Um Zuschreibung handelt es sich dann, wenn ein Sachverhalt oder eine Substanz als für eine andere Substanz charakteristisch dargestellt werden soll. (Biermann 1985: 2)

Hierunter fallen Habeo-Konstruktionen wie a háznak van pincéje ,Das Haus hat einen Keller' (Biermann 1985: 8). Somit basiert das Unterscheidungskriterium von »Possession " und "Zuschreibung" auf derselben wie bei Kenesei/Vago/Fenyvesi (Hg.) 1998 (siehe dazu Kapitel 5.1.1.4), für die attributive Nominalphrase bleibt der Begriff »Possession« bestehen.

Bei den attributiven Possessivkonstruktionen (bei ihr als "possessivische Nominalphrasen, PNP « bezeichnet) unterscheidet Biermann 1985 zwei Typen, (i) eingliedrige und (ii) zweigliedrige; letztere treten in zwei Varianten auf, (ii.a) Juxtaposition innerhalb einer NP und (ii.b) zwei NPn, die "nicht notwendigerweise in Juxtaposition stehen « (Biermann 1985: 31f.). In der Kategorisierung der vorliegenden Arbeit (Tabelle 10) entspricht (i) dabei den Konstruktionstypen 1.b.2 und 1.b.3, das heißt, der Modifizierer wird pronominal oder mit einer Leerstelle realisiert, Typ (ii.a) dem Konstruktionstyp 1.b.1 und (ii.b) Konstruktionstyp (1.c) mit dependent-marking.

In dieser Konstruktion bilden die "zwei Glieder dieses Syntagmas eine $\mathrm{NP}_{\text {«, sind }}$ jedoch in der »Reihenfolge bzw. Position [...]im Satz nicht festgelegt « (Biermann 1985: 33), da head- und dependent-marking den »semanto-syntaktischen Zusammenhang [...] ausreichend markiert « (Biermann 1985: 33). Biermann 1985 systematisiert somit die Possessivkonstruktion anhand der lexikalischen Erwähnung des Modifizierers, der Konstruktionstyp 1.b.2 (pronominaler Modifizierer) wird dabei jedoch nicht berücksichtigt. Die Wahl des Konstruktionstyps hängt von der Vorerwähntheit des »Referenten [...], auf den das Personalsuffix verweist « (Biermann 1985: 33), ab. Biermann 1985 beschreibt die verschiedenen Possessivkonstruktionen des Ungarischen von einem primär syntaktischen Gesichtspunkt, dazu werden jedoch auch Aspekte in Betracht gezogen, welche die pragmatische Ebene der Sprachbeschreibung betreffen; die sogenannte »kommunikative Gliederung der Äußerung « (Biermann 1985: 33) wird als zusätzliche Erklärung für die Unterschiede in zweigliedrigen PNPn herangezogen. Laut Biermann 1985 hat der Modifizierer einer zweigliedrigen $\mathrm{PNP}_{1}$ wie a tanár könyve ,Das Buch des Lehrers' die Funktion eines Determinators, das heißt, die Nominalphrase ist nur als Einheit zugänglich, wohingegen bei einer zweigliedrigen $\mathrm{PNP}_{2}$ wie $a$ tanárnak a könyve ,id. ' beide Konstituenten pragmatische Rollen einnehmen können (Biermann 1985: 33). Den Unterschied begründet Biermann 1985 mit pragmatischen Bedingungen:

Die PNP2 kann anstelle der PNP1 verwendet werden, ohne dass sich der propositionale Gehalt des jeweiligen Satzes ändert. Es handelt sich also um Variation, die jedoch keineswegs frei ist, sondern mit den Regeln der kommunikativen Perspektivengebung korreliert. (Biermann 1985: 33) 
Beide Aspekte, die Determinierung sowie die kommunikative Perspektive, werden im Verlauf der weiteren Beschreibung der Funktionen des Possessivsuffixes zu zentralen Rollen werden, was in den folgenden Abschnitten noch thematisiert wird.

Honti beschreibt in mehreren Aufsätzen die Struktur der prädikativen und attributiven Possessivkonstruktion im Uralischen, zum Beispiel in Honti 2004. Er unterteilt letztere in vier Typen (verglichen Tabelle 9) und verwendet für diese den Begriff der Izafet-Konstruktion (zum Izafet siehe Kapitel 5.2.2.1). Ähnliche Abhandlungen zu diesem Thema sind Honti 2007a; Honti 2007b; Honti 2005 und Honti 2006 thematisieren zusätzlich den möglichen Ursprung beider Konstruktionen.

\subsection{Spezifische Abhandlungen zu Possessivmarkern}

Neben den allgemeinen Überblicken mit Beschreibung der Possessivkonstruktion gibt es auch Abhandlungen zum Possessivsuffix selbst. In der Uralistik befasst sich ein Großteil dieser Aufsätze mit der Morpho-Phonologie sowie mit der Etymologie und historischen Entwicklung des Possessivsuffixes. Hier sind neben Sinor 1978 für das Uralische im Allgemeinen vor allem die Werke von Honti 1976 für das Chantische und Honti 1983 für das Mansische zu nennen.

Das mit Abstand ausführlichste Werk zu Possessivsuffixen ist die 1960 erschienene Monografie von Wolfgang Schlachter »Studien zum Possessivsuffix des Syrjänischen« (im Folgenden Schlachter 1960). Das Werk behandelt somit eine Sprache des permischen Sprachzweiges, soll aufgrund seiner Bedeutung in Hinblick auf den Forschungsgegenstand dennoch im Forschungsstand der vorliegenden Arbeit nicht unberücksichtigt bleiben. Im Vorwort gibt Schlachter unter anderem einen kurzen Einblick in den Forschungsstand und verweist dabei auch auf das Ob-Ugrische, und zwar insbesondere im Hinblick auf die Vorkommen von Possessivsuffixen in Erzählungen. Der Aufbau der Arbeit folgt zwar dem onomasiologischen Muster - die Analyse ist unterteilt in ein Kapitel zur "possessivischen Funktion« (Schlachter 1960: 22-77) und zur »nichtpossessivischen Funktion " (Schlachter 1960: 78-167) - diese sind jedoch ausgehend von der Form, und zwar unterteilt in die einzelnen kodierten Personen aufgebaut, sodass die Possessivkonstruktionen jeweils ein Unterkapitel bilden. Das Kapitel zur »possessivischen Funktion « beginnt mit Beispielen und Erläuterungen zur Verwendung des Possessivsuffixes der ersten Person, dann folgt der Abschnitt mit Beispielen und Erläuterungen zum Possessivsuffix der zweiten Person. Die Beschreibung beider Personalmarker beläuft sich auf insgesamt zehn Seiten und entspricht damit gemeinsam fast dem Umfang der Beispiele und Erläuterungen zum Possessivsuffix der dritten Person. ${ }^{42}$ Im folgenden Kapitel werden die Possessivkonstruktionen beschrieben, jeweils mit eigenen Unterpunkten zu Besitzverhältnissen, Verwandtschaftsbeziehungen und »Bezeichnungen für Körperteile« (Schlachter 1960: 46-49). Der Abschnitt zu Besitzverhältnissen wird als "attributivische Possessivkonstruktion« (Schlachter 1960: 41f.) bezeichnet und behandelt offenbar nur den Konstruktionstyp 1.b.1 und 1.b.2: Es wird auf die Einschränkung auf die dritte Person hingewiesen, sodass mit »attributivisch« der 
als Substantiv realisierte Modifizierer gemeint sein muss, außerdem auf das Erscheinen des Personalpronomens.

Das Kapitel zur »possessivischen Funktion« enthält unter anderem auch einen Abschnitt zu unmarkierten Possessivkonstruktionen (Typ a), sodass insgesamt die Beschreibung der "possessivischen Funktion" eine onomasiologische Beschreibung der adnominalen Possessivkonstruktion mit verschiedenen Konstruktionstypen und den darin möglichen ausgedrückten Kern-Relationen ist. Somit scheint auch der letzte Abschnitt des Kapitels »Beispiele für Funktionsmischung" (Schlachter 1960: 69-77) der vierten Kern-Relation zu entsprechen. Hier wird der Übergang zu den »nicht-possessivischen Funktionen « des Folgekapitels eingeleitet und die erste Person von der weiteren Analyse ausgenommen. In diesem Abschnitt wird auch die »subjektivierende« Funktion der zweiten Person und die »demonstrative Funktion« der dritten Person erwähnt (Schlachter 1960: 73). Diese sind somit den Suffixen inhärent und treten in der »nichtpossessivischen Funktion « gegenüber der "possessivischen « in den Vordergrund (verglichen Schlachter 1960: 83).

Das Kapitel zur »nicht-possessivischen Funktion « beginnt mit Beispielen für »die determinative Verwendung " (Schlachter 1960: 78) des Possessivsuffixes der dritten Person. Erwähnenswert hierbei ist, dass in der »Auswertung « dieser Belege (Schlachter 1960: 83-111) auch der Ausdruck der oben genannten Kern-Relationen erneut erscheint. Somit ist die Unterteilung in "possessivische» und "nicht-possessivische Funktionen" offenbar nicht die Lesart der Relation, sondern der Kosntruktionstyp (1.b.3). ${ }^{43}$ Auch für das Possessivsuffix der zweiten Person werden Belege für die »nicht-possessivische Funktion" im folgenden Abschnitt vorgestellt. In der anschließenden Auswertung (Schlachter 1960: 122-143) wird diese unterteilt in eine determinative, formelhafte, eine Anredefunktion und eine subjektivierende Funktion. Die formelhafte Funktion beinhaltet als »Hauptkennzeichen [...], daß ein deutlicher Zusammenhang zwischen der tatsächlich vorliegenden und der durch die morphologischen Bestandteile angedeuteten Funktion nicht zu erkennen ist (Schlachter 1960: 126). Die subjektivierende Funktion beschreibt das Verknüpfen eines text-internen Referenten mit einem der Sprechaktteilnehmer, nämlich dem Hörer, um den Referenten für diesen salienter zu machen (verglichen Kapitel 5.2):

Der Hörer sieht sich hier [...] einer nach dem freien Gestaltungswillen des Erzählers [...] hinzugefügten Umfärbung der Vorstellung gegenüber, deren Ziel es ist, durch ein fingiertes Zugehörigkeitsverhältnis dem Hörer die Vorstellung als zu seinem augenblicklichen Bewußtseinsbereich gehörig näher zu bringen. (Schlachter 1960: 131$)^{44}$

43 Diese Annahme beruht einzig und allein auf den Beobachtungen der Verfasserin, und zwar vor allem abgeleitet von den jeweiligen Beispielen zu Beginn der einzelnen Abschnitte. Da das System der Possessivsuffigierung im Komi-Syrjänischen jedoch Unterschiede zu dem der ugrischen Sprachen aufweist (z. B. Abweichungen in der Suffixabfolge je nach Kasus, Zusammenfall von Cx und Px zu einer Art Portemanteau-Morphem im Akkusativ) und die Beispiele eine detaillierte Glossierung vermissen lassen, kann darüber keine gesicherte Aussage getroffen werden.

44 Ein solcher Mismatch wie in der formelhaften und subjektivierenden Funktion beschrieben wird u. a. auch für das Chantische beschrieben (Nikolaeva 2003: 8) und kommt in einigen wenigen Korpus-Belegen des Kazym-Chantischen ebenfalls vor. 
Das letzte Hauptkapitel der Monografie widmet sich den Postpositionen mit Possessivsuffixen und beinhaltet ebenfalls einen Abschnitt zum Vergleich mit dem »suffixlosen Gebrauch «(Schlachter 1960: 214-218). Formen der ersten Person werden in diesem Kapitel ebenfalls nicht analysiert.

Die Monografie von Schlachter kann aufgrund ihres Alters und der Weiterentwicklung in der Forschung seit ihrem Erscheinen nur bedingt als Quelle und Forschungsgrundlage herangezogen werden: Die Darstellung der Beispiele entspricht nicht dem heutigen Verfahren, sodass nicht nur das Fehlen einer Wort-für-Wort-Glossierung, sondern vor allem die Repräsentation der jeweiligen Belegstellen des Korpus lediglich in Form der deutschen Übersetzung im Fließtext eine Auseinandersetzung mit den Belegen erschwert. Hinzu kommen veraltete Begrifflichkeiten bzw. werden heutzutage für die hier beschriebenen Erkenntnisse andere Termini verwendet. Das Fehlen mancher Definition der verwendeten Begrifflichkeiten erschwert zusätzlich die Einordnung und Adaption des Forschungsstandes. Andererseits darf man nicht unterschlagen, dass die Arbeit zum Zeitpunkt ihrer Veröffentlichung ein Novum in der Forschung dargestellt haben muss:

Für den Sprachforscher bedingt sie in zweifacher Richtung eine Abweichung von dem in der Linguistik immer noch am meisten geübten historischen Verfahren: einerseits fordert sie eine besonders sorgfältige, ausführliche Analyse der Texte [...]; andererseits nötigt sie zu einer Beschränkung der sprachwissenschaftlichen Fragestellung im engeren Sinne, insofern die Folgerungen, die sich aus einem so speziell gelagerten Material ergeben, sich nicht ohne weiteres auf anderes Material oder gar auf andere Sprachen übertragen lassen. (Schlachter 1960: 9)

Wie die Schilderung im Verlauf dieses Kapitels aufzeigen wird, bleibt die korpus-basierte, sprachspezifische Abhandlung zum Possessivsuffix von Schlachter mit wenigen Ausnahmen bis heute einzigartig. Seine schon vor 55 Jahren festgehaltenen Beobachtungen zu pragmatischen Gesichtspunkten in der Verwendung der Possessivsuffixe sind erst in den neueren Abhandlungen ein fester Bestandteil der Forschungsgrundlage geworden (zu den neueren Forschungsansätzen, siehe Kapitel 2.3.4). Und auch die Berücksichtigung der Kategorie Person ist seither nicht vergleichbar in einer anderen Arbeit erfolgt.

Die Monografie »Verteilung und Leistung der Personalaffixe im Ungarischen« von Christoph Reichert (im Folgenden Reichert 1986) ist vermutlich die bislang einzige Abhandlung, welche sich mit dem Possessivsuffix des Ungarischen in einem eher semasiologischen Forschungsansatz befasst. Im einleitenden Kapitel thematisiert Reichert das Problem der Distinktion einzelner Wortarten im Ungarischen, was sowohl in Zusammenhang mit dem gemeinsamen Ursprung mit den verbalen Personalmarkern und der Unterscheidung der einzelnen Konstruktionstypen als auch der Bestandteile der einzelnen Konstruktionen mit Possessivsuffixen eine Rolle spielt, so auch in der vorliegenden Arbeit (Kapitel 1.2 und 5.1.). Des Weiteren wird im ersten Kapitel die morpho-phonologische Variation der Personalmarker aufgrund von Vokalharmonie oder Stammveränderungen thematisiert (Reichert 1986: 6-13).

Das zweite Kapitel (Reichert 1986: 14-80) befasst sich mit der »Verteilung der Personalsuffixe« (Reichert 1986: 14), das heißt, die einzelnen Paradigmen der Personalmarker 
werden nach der Wortart, welcher sie angefügt werden, kategorisiert. Auf die Possessivsuffixe wird dabei zum ersten Mal in Zusammenhang mit dem Infinitiv verwiesen: Die Personalsuffixe des Infinitivs gehören »formal zum Bereich der nominalen Endungen « (Reichert 1986: 41). Nach Vorstellung des Paradigmas folgt eine Übersicht über die »Verwendung personalsuffigierter Infinitive« (Reichert 1986: 41-45). Es handelt sich dabei um Modalverbkonstruktionen (mehr hierzu unter Kapitel 5.1.2.1). Erwähnenswert hierbei ist, dass Reichert die Personalmarker am Infinitiv unter anderem deswegen nicht zu den verbalen Personalmarkern zählt, da diese "ggf. einen zusätzlichen Objektbezug auf[weisen] (objektive Konjugation)«(Reichert 1986: 43). Somit nimmt Reichert für die Konstruktion VN-Px an, dass lediglich ein Referent im Possessivsuffix kodiert wird, wie es auch die vorliegende Arbeit postuliert. ${ }^{45}$ Das Paradigma der »Personalsuffixe am Partizip Perfekt « (Reichert 1986: 45-52) ist laut Reichert mit dem der Infinitive identisch (Reichert 1986: 45). Auch dieses Kapitel beinhaltet eine Übersicht zur Verwendung, Partizipialkonstruktionen werden nach ihrer Funktion als Attribut, zum Beispiel az Mikulás hozta csokoládé, die vom Nikolaus gebrachte Schokolade' (Reichert 1986: 47) und als Nomen Actionis unterteilt (Beispiel 17). Beispiele für letzteres entsprechen eher den Nebensatzkonstruktionen und nicht der Definition des »Nomen Actionis« der vorliegenden Arbeit (siehe Kapitel 5.1.2).

(17) Ungarisch, Reichert 1986: 50

$\begin{array}{llllllll}j 0 ̈ & -t t & -o ̈ m & -b e n & \text { meg- } & \text { ijed } & -t & -e m \\ \text { come } & \text {-PTCP.PST } & -1 S G & -I N E & \text { PFV } & \text { to startle } & -P S T & -1 S G\end{array}$

'Als ich ankam, erschrak ich.'

Danach folgen die »Personalsuffixe am Nomen« (Reichert 1986: 52-62), wobei hiermit die Konstruktion Su Bs-Px gemeint ist. Nach einer exemplarischen Segmentierung aller möglichen Bestandteile der Deklination des Substantives erfolgt eine Darstellung des Paradigmas der Possessivsuffixe und es wird auf die Bildung des Mehrbesitzes eingegangen. Erwähnenswert hierbei ist, dass die Kodierung des Mehrbesitzes bei Reichert als »Nomen im Plural « bezeichnet wird und das Zeichen für den Mehrbesitz - $i$ - zum Stamm und nicht zum Suffix gerechnet wird. Die Formen des Mehrbesitzes werden ohne den Numerusmarker dann als eigenes Paradigma angesehen. Folglich wird auch nicht von einer Leerstelle als Kodierung des Einbesitzes ausgegangen (verglichen Kapitel 1.1), welche bei den Konstruktionen mit Verbalnomina und Postpositionen entfällt und in der Glossierung entsprechend vermerkt wird (verglichen Kapitel 4.2.1). Die Glossierung von Reichert berücksichtigt lediglich den als Possessor bezeichneten Referenten, wie es in vielen Abhandlungen der Fall ist. ${ }^{46} \mathrm{Ob}$ und wie die Kodierung des Mehrbesitzes in der Glossierung berücksichtigt werden würde, lässt sich nicht feststellen, da keine Beispiele im Mehrbesitz aufgeführt werden. Bei der objektiven Konjugation hingegen wird die Mit-Kodierung eines zweiten Referenten zumindest mit der Glosse ».obj« kenntlich gemacht. Die Verwendung der nominalen Personalmarker wird unterteilt in »Verwendung in der Possessiv-Nominalphrase " (Reichert 1986: 56-59) und »in der Zuschreibungskonstruktion« (Reichert 1986: 59-62). Damit orientiert sich die

45 Allerdings wird auch im weiteren Verlauf der Abhandlung nicht auf die Kodierung des Possessum im Possessivsuffix eingegangen.

46 Z. B. Abondolo 1998a, Nikolaeva 2003, Simonenko 2014; siehe dazu auch Kapitel 4.2.1. 
Systematisierung und Benennung an Biermann 1985. Zur Possessivnominalphrase wird auf die verschiedenen syntaktischen Rollen, welche diese im Satz einnehmen kann, eingegangen sowie auf die Realisierung des Modifizierers in der Konstruktion. Hier spricht Reichert analog zu Biermann 1985 von einteiliger (entspricht Konstruktionstyp 1.b.3 in der vorliegenden Arbeit) und zweiteiliger PNP (entspricht Konstruktionstyp 1.b.1 und 1.b.2 der vorliegenden Arbeit). Demzufolge wird hier abgesehen von einem Hinweis auf die »emphatische" (Reichert 1986: 56) Verwendung des Personalpronomens ebenfalls keine Unterscheidung zwischen Realisierung des Modifizierers als Substantiv oder Pronomen getroffen, außerdem entfällt ein Hinweis auf einen als Leerstelle realisierten Modifizierer (da dieser Konstruktionstyp als »einteilig« bezeichnet wird). Weiterhin wird auf die Inkongruenz bei der Wahl des Possessivsuffixes bei substantivischen Possessoren im Plural hingewiesen (verglichen Kapitel 1.2.1) und auf Konstruktionen mit mehreren Possessoren.

Es wird zudem auf die Überschneidung der Wortart Substantiv und Postposition einiger Formen eingegangen, welche auch in der vorliegenden Arbeit thematisiert wird (verglichen Kapitel 5.1.3.2). Erwähnenswert ist der Hinweis auf »lexikalisierte Bildungen« (Reichert 1986: 58), das heißt Possessivkonstruktionen vom Typ Subs Subs-Px, welche unter Umständen als Kompositum grammatikalisiert sind. ${ }^{47}$ Auf das dependentmarking wird in Bezug auf die Wortstellung eingegangen und auf die »kommunikative Gliederung« von Biermann 1985 verwiesen. Im Abschnitt zur Zuschreibungskonstruktion erscheinen Beispiele der Habeo-Konstruktion, und es wird ein Bezug zur Infinitivkonstruktion in Hinblick auf die Struktur hergestellt. Auf das Paradigma des Possessivsuffixes wird nicht weiter eingegangen. Der Abschnitt zu »Personalsuffixen an Pronomina« (Reichert 1986: 62-70) beinhaltet die Reflexiv-, Possessiv- und Personalpronomina im Akkusativ. Der vorletzte Abschnitt widmet sich sowohl »Kasuselementen « als auch Postpositionen als Kopf der Konstruktion (Reichert 1986: 71-77). Damit fasst Reichert Postpositionen und die affigierten Formen der Personalpronomen zu einem Konstruktionstyp zusammen (siehe hierzu Kapitel 5.1.3.1). Den letzten Abschnitt bilden »Personalsuffixe an Numeralen« (Reichert 1986: 77), welche »ausschließlich substantivisch gebraucht « werden können (Reichert 1986: 78). Hier sind besonders die Bruchzahlen hervorzuheben sowie die davon abgeleitete Konstruktion der Datumsangabe, welche Possessivsuffixe aufweisen. ${ }^{48}$

47 Das hier aufgeführte Beispiel tanácsháza, Rathaus` findet sich tatsächlich 55 mal (0,29 Vorkommen pro einer Millionen Wörter) als zusammengesetzte Form im Ungarischen Nationalkorpus (HNC), davon sind fast alle als Substantiv (N.NOM) ohne explizite Glossierung des Possessivsuffixes getaggt (HNC_ Abfrage_0037.pdf). Siehe hierzu auch Kapitel 5.2.2.1. Zum HNC siehe Kapitel 4.1.2.

48 Reichert 1986 weist darauf hin, dass prinzipiell alle Personen des Possessivsuffixes an Bruchzahlen angefügt werden können, jedoch vorwiegend Belege der dritten Person Singular auftreten (Reichert 1986: 77f.). Die Verwendung der Possessivsuffixe mit Bruchzahlen lässt sich meines Erachtens mit einer TeilGanzes-Relation erklären. Das Korpus der vorliegenden Arbeit kann den Konstruktionstyp NUM-Px jedoch aus Mangel an Belegen nicht weiter thematisieren. 
Das dritte Kapitel (Reichert 1986: 81-106) widmet sich der »Leistung der Personalaffixe im Ungarischen« (Reichert 1986: 81), diese Leistung definiert Reichert dabei wie folgt:

Unter `Leistung « soll dabei der umfassende Beitrag, den die PSe [Personalsuffixe, GJ] zur Mitteilung, also zur kommunikativen Funktion einer Äußerung leisten, verstanden werden. Diese Leistung setzt sich aus verschiedenen Einzelaspekten zusammen, wie zum Beispiel der relationierenden und damit verbunden der Kongruenzfunktion, der anaphorischen und der deiktischen Funktion, sowie der Mitkodierung der syntaktischen Funktion der Bezugskonstituente [...]. (Reichert 1986: 81)

Das Kapitel beginnt mit einer Definition der Begriffe Kongruenz, Anaphorik und Deixis, wobei Reichert von einer Unterscheidung zwischen »Kongruenz- und anaphorischen Bezügen« (Reichert 1986: 81) ausgeht.

Als Kongruenzbezüge bezeichnet Reichert die Markierung der Zugehörigkeit von Kopf und Modifizierer innerhalb der Possessivkonstruktion (entspricht Konstruktionstyp 1.b.1, 1.b.2, 1.b.4 und 1.c), als anaphorischen Bezug den Verweis außerhalb der Konstruktion (entspricht Konstruktionstyp 1.b.3). Erstere bezeichnet er als syntaktische, letztere als semantische Repräsentation und reiht beide in ein Kontinuum (»Skala der Anapher « Reichert 1986: 82) ein, dessen jeweilige Enden die explizite und die implizite Anapher bilden (siehe dazu Kapitel 3 und 5.2). Abschließend widmet sich Reichert der Definition der Deixis (siehe dazu Kapitel 3.2.2). Schließlich werden die einzelnen Leistungen beschrieben, beginnend mit der »relationierenden Leistung«. Darunter versteht er zum Beispiel die Relation von Possessor und Possessum (ausgedrückt durch die Possessivsuffixe), aber auch zwischen Subjekt (sowie Objekt) und Prädikat (ausgedrückt durch verbale Personalmarker und Possessivsuffixe) und ebenso die Kasusrektion (ausgedrückt durch Kasussuffixe und Postpositionen). Danach beschreibt er solche Konstruktionen, in welchen der Modifizierer als Leerstelle realisiert wird (zum Beispiel Konstruktionstyp 1.b.3) als zwischen Kongruenz und Anapher stehend (Reichert 1986: 93f.), da sie zwar wie bei den relationierenden Konstruktionen die Kongruenz zwischen Modifizierer (»Trägerkonstituente« Reichert 1986: 94) und Kopf anzeigt, der Modifizierer jedoch nicht explizit realisiert wird. Somit hat das Personalzeichen gleichzeitig die anaphorische Funktion eines Pronomens (siehe hierzu auch Kapitel 3). Der folgende Abschnitt widmet sich der Bildung der Personalpronomina im Ungarischen, worauf ein Abschnitt zur Form der Personalsuffixe folgt. Reichert gibt an, insgesamt 19 Paradigmen von Personalsuffixen in Kapitel 2 vorgestellt zu haben. Dem Paradigma der Possessivsuffixe entsprechen dabei die zwei nominalen Paradigmen (Einbesitz- und Mehrbesitzformen), die zwei pronominalen Paradigmen, das Paradigma des Infinitivs, des Partizip Perfekts sowie das Paradigma der Kasus und Postpositionen und das der Numeralia. Diese ordnet er zwei Grundtypen zu, dem Paradigma der Possessivsuffixe im Einbesitz und dem Paradigma der Possessivsuffixe im Mehrbesitz. Ein letzter Abschnitt widmet sich der Entstehung des Systems der Personalmarker im Ungarischen. Im folgenden Schlusskapitel (Reichert 1986: 107f.) fasst Reichert noch einmal die insgesamt fünf Grundtypen (drei verbale, zwei nominale) der Personalmarker zusammen, und auch hier wird noch einmal explizit auf den »Einfachbezug « (Reichert 1986: 107) der nominalen Personalmarker verwiesen. Der Begriff »Possessivsuffix « wird in der gesamten Abhandlung nicht verwendet. 
Mit Ausnahme von Reichert 1986 haben Possessivsuffixe als morpho-syntaktischer Bestandteil der Possessivkonstruktionen, das heißt unter dem Gesichtspunkt der formalen Realisierung, demnach längst nicht dieselbe intensive Beschreibung erfahren wie andere Bestandteile - weder in der Uralistik selbst noch in der Linguistik allgemein. Stattdessen sind insbesondere die Markierung mittels Kasussuffix (das heißt dependent-marking mit dem Genitiv-Kasus) sowie die in den indo-europäischen Sprachen bekannten Possessivpronomina hier im Fokus der Forschung.

Eine der neuesten Publikationen, »Morphosyntactic Categories and the Expression of Possession« (Börjars/Denison/Scott (Hg.) 2013), befasst sich mit adnominalen Possessivkonstruktionen und deren Markern, wobei im Mittelpunkt der Abhandlung der Genitiv des Englischen steht. Die weiteren Aufsätze ordnen sich thematisch an und handeln fast ausschließlich über vergleichbare Strukturen in germanischen Sprachen. Ein einziger Aufsatz befasst sich mit Izafet-Konstruktionen im Urdu (indo-arischer Sprachzweig der indo-europäischen Sprachfamilie, in Indien und Pakistan gesprochen, strukturell fast identisch mit Hindi; verglichen Bögel/Butt 2013: 291). Somit wird in diesem Band der morpho-syntaktischen Ausdrucksmöglichkeit der Dimension Possession intensiv Beachtung geschenkt, da es sich aber um Kasusmorphologie indo-europäischer Sprachen handelt, sind diese Ansätze nicht ohne Weiteres auf Possessivsuffixe abzuleiten, welche anders als ein Genitiv zusätzlich die Kategorie Person mitkodieren.

Der »World Atlas of Language Structure Online» (Dryer/Haspelmath (Hg.) 2013) befasst sich in vier Kapiteln mit Aspekten der Possession bzw. Possessivität in Nominalphrasen und konzentriert seine Beschreibung ebenfalls auf die morphologische Markierung: Es existiert ein Kapitel zu pronominalen Possessivaffixen und deren Position (im Kapitel wird auf keine uralische Sprache hingewiesen, auf der zugehörigen Karte 57A wurde jedoch sowohl Chantisch, Mansisch als auch Ungarisch der Wert "possessive suffixes « zugewiesen, verglichen Dryer 2013). Ein Kapitel widmet sich dem »locus" der Markierung, das heißt dem head- und dependent-marking (Nichols/Bickel 2013a): Im Kapitel wird nicht auf uralische Sprachen verwiesen, auf der zugehörigen Karte 27A wird Ungarisch der Wert head-marking zugewiesen, die ob-ugrischen Sprachen fehlen vollständig auf der Karte, verglichen Nichols/Bickel 2013a). Auch im Kapitel zur Obligatheit der Markierung werden die uralischen respektive ugrischen Sprachen nicht erwähnt, auf der zugehörigen Karte 58A wird eine obligatorische Markierung mit Possessivsuffix für das Ungarische mit dem Wert »absent « vermerkt, die ob-ugrischen Sprachen fehlen hier ebenfalls, verglichen Bickel/Nichols 2013), genauso wie im Kapitel 59 »Possessive classification « (Nichols/Bickel 2013b).

Während das Fehlen der ob-ugrischen Sprachen auf den Karten zu den Kapiteln 58 und 59 mehr oder weniger nachvollziehbar ist - sowohl das Fehlen einer nicht-possessiven Wortform ${ }^{49}$ als auch das Fehlen mehrerer nominaler Possessivkonstruktionen und damit einhergehender Restriktionen in der jeweiligen Verwendung sind weder für das Ungarische noch für die ob-ugrischen Sprachen in einem Umfang attestierbar, welcher zur Klassifizierung herangezogen werden könnte. Lediglich die inkonsequente Auflistung von Sprachen, denen der jeweilige Wert fehlt, wäre hier anzumerken. Im

49 Hier geht es um die obligatorische Markierung mit Possessivmarkern z. B. bei Verwandtschaftstermini, näheres dazu Kapitel 5.1.1.4. 
Falle der Position der Markierung (Nichols/Bickel 2013a) jedoch ist das Fehlen eines Hinweises zum head-marking der ob-ugrischen Sprachen meines Erachtens ein Fehler. Abschließend soll auch darauf hingewiesen werden, dass es sich bei den für die ugrischen Sprachen herangezogenen Quellen fast ausschließlich um Daten aus den Handbüchern (Sinor (Hg.) 1988, Collinder 1957) und Grammatiken/Chrestomathien (zum Beispiel Kenesei/Vago/Fenyvesi (Hg.) 1998; Rédei 1965; Murphy 1968) handelt, welche mit Ausnahme von Filchenko 2007 allesamt nicht neueren Datums sind und die eingangs beschriebenen Einschränkungen in der Beschreibung der Possessivsuffixe aufweisen. Fachspezifische detaillierte Studien (wie zum Beispiel Schlachter 1960) fehlen ebenfalls.

\subsubsection{Exkurs: Areallinguistik}

Wie der Titel »World Atlas of Language Structure Online« schon vermuten lässt, handelt es sich dabei um eine typologische Beschreibung linguistischer Merkmale, deren Notation mittels bestimmter Werte das jeweilige Vorkommen in den Sprachen der Welt areal darstellt. Dieser Forschungsaspekt der arealen Verteilung bei Possessivkonstruktionen und die damit verbundene mögliche gegenseitige Beeinflussung benachbarter Sprachen ist insbesondere im eurasischen Sprachraum ein Forschungsbereich, in welchem auch immer wieder die uralischen Sprachen in einem gewissen Umfang mitberücksichtigt werden, zum Beispiel bei Manzelli 1990.

In diesem Aufsatz erhebt Manzelli 1990 den Anspruch, eine typologische Beschreibung von freien und gebundenen nominalen Possessivmarkern in nicht weniger als allen europäischen Sprachen (mit dem Ural als Ostgrenze) zu leisten. Als typologische Studie soll es dabei nicht um Possession nach Seiler 1983a gehen, sondern um adnominale Modifizierer, welche zum Ausdruck von Possession verwendet werden (Manzelli 1990: 63).

Aus der uralischen Sprachfamilie kommen dabei auf seiner Referenzkarte (»reference map«) Saamisch, die ostsee-finnischen Sprachen, Mordwinisch, Mari, der permische Sprachzweig, Ungarisch sowie (das westlich des Urals gesprochene) Nenzisch $^{50}$ vor (Manzelli 1990: 64f.); des Weiteren weist Manzelli 1990 auf das Vorkommen von Possessivsuffixen in den östlich des Urals gesprochenen uralischen Sprachen hin (Manzelli 1990: 72). Unter den Sprachen, welche über Possessivsuffixe verfügen, ist als uralischer Vertreter jedoch nur das Ungarische erwähnt (Manzelli 1990: 66, 76), in einem anderen Punkt weist er dazu auf den Schwund der Possessivsuffixe in den ostsee-finnischen Sprachen hin - mit dem Hinweis, dass dies eine Tendenz sämtlicher

50 Die Legende seiner Referenzkarte ist einerseits sehr detailliert, er listet sowohl Erza- und MokshaMordwinisch, Berg- als auch Wiesen-Mari einzeln auf, sowie die drei wichtigsten Sprachen des permischen Sprachzweigs (Komi-Permjakisch, Komi-Syrjänisch und Udmurtisch). Auf der anderen Seite jedoch wird Saamisch mit einem Eintrag gelistet, betitelt als Norwegisch-Saamisch (»Norwegian Lappish«) (Manzelli 1990: 64f.). Dies ist keine in der Uralistik geläufige Bezeichnung, gemeint wird vermutlich Nord-Saamisch sein, aller Wahrscheinlichkeit nach der Finnmark-Dialekt (verglichen Sammallahti 1998: 51). 
finnischer Sprachen, aber nicht ugrischer sei ${ }^{51}$ (Manzelli 1990: 70). Der Aufsatz umfasst insgesamt 48 Seiten, wovon die ersten 13 Seiten aus kurzen Zusammenfassungen zu den behandelten Parametern sowie einer Referenzkarte inklusive Legende bestehen. Der Rest des Aufsatzes besteht aus diversen Tabellen, auf welche in den einzelnen Abschnitten verwiesen wird. Tabelle 16 »Diagnostic list« erstreckt sich über mehr als zwölf Seiten inklusive Anmerkungen (Manzelli 1990: 84-96) und bildet die Datengrundlage zum Hauptaugenmerk seiner Abhandlung: der Voran- oder Nachstellung possessiver Modifizierer in der Nominalphrase (Manzelli 1990: 70). Eine detaillierte Behandlung der Possessivsuffixe in uralischen Sprachen kann angesichts der Menge der analysierten Sprachen und Parameter, der relativen Kürze des Aufsatzes und den konsultierten Quellen nicht erwartet werden - Manzellis Daten zu den uralischen Sprachen stammen ebenfalls neben einigen Grammatiken und Chrestomathien hauptsächlich aus den gängigen Handbüchern der Uralistik: Sinor (Hg.) 1988, Décsy 1965, Lytkin/Majtinskaja (Hg.) 1966, aus Collinder 196952, Tauli 1966, Laanest 1982 und aus Benkö/Samu (Hg.) 1972 für das Ungarische, welche allesamt ausgerichtet sind, einen breiten Überblick über die gesamten linguistischen Eigenschaften der jeweiligen Sprache(n) zu geben. Einzig für das Finnische wurde ein Aufsatz, der sich spezifisch mit dem Thema Possessivsuffixe befasst, herangezogen (Pierrehumbert 1980), die Monografie von Schlachter 1960 hingegen auch bei Manzelli 1990 nicht.

Andere Aufsätze, die sich mit Konstruktionstypen und arealer Verteilung befassen, sind zum Beispiel Koptjevskaja-Tamm 2002 und Koptjevskaja-Tamm 2003.

Irina Nikolaeva setzt in ihrem Aufsatz von 2001 (Nikolaeva 2001) die externen Possessivkonstruktionen des Ungarischen in einen uralischen sowie europäischen Kontext und folgt damit auch dem Forschungsgegenstand von Haspelmath 1999. Da die externe Possession an sich nicht Gegenstand der vorliegenden Arbeit ist (siehe Kapitel 1.2.2), wird von einer detaillierteren Analyse des Forschungsstandes hier abgesehen.

\subsubsection{Possessivpronomina}

Auch im Forschungsbereich der pronominalen Marker in Possessivkonstruktionen ist das Possessivsuffix in weit geringerem Umfang Gegenstand der Beschreibung als beispielsweise Possessivpronomina, wie sie in den indo-europäischen Sprachen vorkommen. Die dort gewonnenen Erkenntnisse lassen sich durchaus zu einem gewissen Grad auf die Konstruktionen mit Possessivsuffixen ableiten, dürfen jedoch keinesfalls mit diesen gleichgesetzt werden. Dennoch findet sich vielmals in den Abhandlungen zu Possessivpronomen in einem Nebensatz ein Hinweis auf Suffixe, womit suggeriert wird, dass deren Funktionsweise eins zu eins übertragbar wäre. Beispiele, wie dieses Schema adaptiert wurde, sind Majtinskaja 1970 und Schmidt 1988. Possessivsuffixe

51 Es ist davon auszugehen, dass er sich mit den Bezeichnungen finnic und ugric auf die unter dem Begriff fenno-permisch subsumierten Sprachzweige einerseits sowie auf den ugrischen Sprachzweig andererseits bezieht und damit in ersterer Gruppe auch Mari und die permischen Sprachen mit einbezieht, welche ein nachweislich sehr produktives System der Possessivsuffixe aufweisen, siehe z. B. Schlachter 1960.

52 Zweite, überarbeitete Auflage von Collinder 1957. 
haben sich zwar auch in den uralischen Sprachen wie auch andere Personalmarker aus Pronomen weiterentwickelt; die Grammatikalisierung geht jedoch neben Formwandel immer mit Funktionswandel einher (Bußmann 2002: 260), sodass allein aus diesem Grund die Gleichsetzung mit Possessivpronomen meines Erachtens falsch ist. Sie können als Ausgangspunkt und Wegweiser Anregungen geben, in welchen Bereichen nach Funktionen der Possessivsuffixe gesucht werden kann, jedoch nicht als Beschreibungsrahmen für die Forschung.

\subsubsection{Die Definitheits-Theorie}

Wie die bisherige Zusammenfassung des Forschungsstandes gezeigt hat, ist die Bearbeitung der Dimension Possession oftmals sowohl in der Typologie als auch innerhalb der Uralistik selbst basierend auf oder im Vergleich zur Realisierung von Possession in den indo-europäischen Sprachen angelegt. Darin begründet sich nicht nur die Terminologie des Possessivsuffixes, sondern auch der Forschungsansatz über Possessivsuffixe als Bestandteil einer Konstruktion, welche Besitzrelationen ausdrückt.

Wie bereits erwähnt subsumiert der linguistische Begriff »Possession« eine bestimmte Relationen zwischen zwei Entitäten, welche sich für die meisten Sprachen belegen lassen, sprachspezifische Unterschiede sind mit Bezeichnungen wie »Assoziation jeglicher Art« und dergleichen klassifizierbar (verglichen Kapitel 1.2.2.1). Allen in der Possessivkonstruktion ausgedrückten Relationen gemeinsam ist hier die Annahme eines Possessors.

In den uralischen Sprachen jedoch, scheitert dieser Ansatz als Erklärungsgrundlage für sämtliche Verwendungen des Possessivsuffixes in attributiven Possessivkonstruktionen, von Verwendungen in anderen Konstruktionen (mit Postpositionen oder infiniten Verbalformen) ganz zu schweigen:

(18) Komi, Simonenko 2014: $2^{53}$

$\begin{array}{lllll}\text { šond } & -y s & \text { dep } & - \text { ši } & -s \\ \text { sun } & -3 S G & \text { dep } & \text {-DETR } & \text {-PRT.3SG }\end{array}$

'The sun has set.'

Nimmt man für einen Beleg wie Beispiel (18) einen belebten Referenten als konkreten Besitzer an, so kann man hier keinen möglichen Referenten zuordnen. Dieser Argumentationsverlauf wird bis heute herangezogen, um den Possessivsuffixen in den uralischen Sprachen eine zweite, sogenannte »nicht-possessive« oder »nicht-prototypische Funktion « zu attestieren. Vielen Aufsätzen dienen dabei die Definitheits-Kriterien nach Hawkins 1978 als Basis der Argumentation (zur Definitheits-Theorie und zum Possessivsuffix in Detail siehe Kapitel 3.4). Seit Mitte des letzten Jahrhunderts wird dieser

53 Dieses Beispiel entspricht einem Set von Verwendungen des Possessivsuffixes der dritten Person Singular, welches durchgehend in den Arbeiten zur Definitheits-Theorie als Argumentation herangezogen wird. Zur Illustration musste auf ein Beispiel aus dem Komi zurückgegriffen werden, da sich in den einschlägigen Quellen, welche in ihrer Argumentation auch die ob-ugrischen Sprachen mit einbeziehen, kaum ein Beispiel aus dem Ob-Ugrischen finden lässt. Auch das Korpus der vorliegenden Arbeit weist kaum derartige Belege auf. Notation und Glossierung nach Simonenko 2014. 
Bereich mit dem Aspekt der Definitheit in der Forschung innerhalb der Uralistik und in der allgemeinen Typologie in Verbindung gebracht, so verweisen die älteren Handbücher und Monografien gerne auf die determinierende Funktion des Possessivsuffixes, welche mit einem definiten Artikel zu vergleichen sei, zum Beispiel bei Collinder 1957 (Collinder 1957, 322, 349; Collinder 1955, 203), Tauli 1966 (zum Beispiel Tauli 1966, 148) oder Hajdú/Domokos (Hg.) 1987 (223).

Es existieren jedoch auch Abhandlungen neueren Datums, sodass die Gemeinsamkeiten zwischen Definitheit und Possession ein häufig thematisierter Forschungsgegenstand auch der letzten 15 Jahre war und ist. Mit der Grammatikalisierung von Possessivsuffixen zu Definitheitsmarkern befasst sich zum Beispiel Fraurud 2001.

In ihrem Aufsatz »Possessives with extensive use: A source of definite articles? « (im Folgenden Fraurud 2001) befasst sich Kari Fraurud mit einer möglichen Grammatikalisierung der Possessivsuffixe in altaischen und uralischen Sprachen zu definiten Artikeln. Gleich in der Einleitung weist sie auf den genauen Konstruktionstyp hin, welcher in Zusammenhang mit der Grammatikalisierung zum Definitheitsmarker als einziger in Frage kommt: Der Aufsatz befasst sich somit mit der Possessivkonstruktion vom Typ 1.b.3 in der dritten Person Singular. Der zweite Abschnitt (Fraurud 2001: 244-248) stellt die Ausgangsbeobachtungen dar, welche zur Annahme einer Grammatikalisierung zum definiten Artikel im Allgemeinen herangezogen werden. Diese sind die strukturelle Ähnlichkeit in der diachronen Entwicklung von Demonstrativa und definiten Artikeln, der synchronen Überschneidung im Gebrauch von Demonstrativa, definiten Artikeln und Possessivmarkern und der nicht-prototypische Gebrauch von Possessivmarkern in manchen Sprachen (Fraurud 2001: 244). Erwähnenswert hierbei ist, dass die Beschreibung der ersten beiden Ausgangsbeobachtungen Beispiele aus vorwiegend indo-europäischen Sprachen beinhaltet, während die Beschreibung des dritten Aspekts vorwiegend die uralischen und altaischen Sprachen betrifft, sodass sich schon hier die generelle Tendenz, die Eigenschaften der indo-europäischen Sprachen als Basis für die Beschreibung der ural-altaischen Merkmale des Possessivsuffixes heranzuziehen, widerspiegelt.

Abschnitt Drei (Fraurud 2001: 248f.) formuliert die Hypothesen. Diese sind erstens eine Grammatikalisierung vom Possessivsuffix (der dritten Person Singular Einbesitz) zum definiten Artikel, ähnlich den Demonstrativpronomina. Und zweitens ist der Ausgangspunkt der Grammatikalisierung beim Possessivsuffix die assoziativanaphorische Verwendung und nicht die anaphorische Verwendung (zu den Kriterien von Hawkins 1978 siehe Kapitel 3.4) wie beim Demonstrativpronomen (Fraurud 2001: 249). Im vierten Abschnitt (Fraurud 2001: 250-253) werden Argumente für die Hypothese einer Grammatikalisierung zum definiten Artikel aufgeführt. Diese sind (i) der Formwandel einhergehend mit der Grammatikalisierung von freien Morphemen (Personalpronomina) zu gebundenen Klitika (Possessivsuffixen) ähnlich der Entwicklung von Demonstrativpronomen, (ii) die hohe Frequenz im Gebrauch und (iii) die erweiterte (»extended «) Verwendung des Possessivsuffixes in einer assoziativ-anaphorischen Funktion. Abschnitt Fünf (Fraurud 2001: 253-258) beinhaltet die Argumente gegen diese Hypothesen.

Diese sind (i) die fehlende Obligatheit der Markierung, (ii) eine gewisse zeitliche Stabilität in der beobachteten Verwendung (der nicht-possessive Gebrauch wird für die 
uralischen Sprachen bis ins Ur-Uralische zurückdatiert, sodass es sich nicht um eine Weiterentwicklung jüngeren Datums handeln kann), (iii) die gleichzeitige Verwendung in den sogenannten prototypischen und nicht-prototypischen Verwendungen und (iv) der ebenfalls häufige und teilweise gleichzeitige Gebrauch der Demonstrativpronomina in einer vergleichbaren Funktion. Der abschließende Abschnitt (Fraurud 2001: 258-262) gibt eine Zusammenfassung der Erkenntnisse und gelangt zu der Schlussfolgerung, dass weder eine Grammatikalisierung zum definiten Artikel stattgefunden hat noch vermutlich jemals stattfinden wird (Fraurud 2001: 262). So sind unter anderem auch andere Verwendungen nach den Kriterien von Hawkins 1978 für die Possessivsuffixe in mehreren uralischen Sprachen zu belegen, sodass die zweite Hypothese hier zu eingeschränkt ist, oder auch die Entwicklung einer neuen Strategie, die linguistische Dimension auszudrücken und die Possessivkonstruktion damit zu ersetzen, fehlt. ${ }^{54}$ Der parallele Gebrauch ist sogar bis in das Ur-Uralische belegt (Fraurud 2001: 260). Dabei weist sie unter anderem auf die Schwierigkeit einer gründlicheren Forschung aufgrund der fehlenden Daten sowohl diachron als auch im Diskurs-Zusammenhang hin. Abschließend bringt sie den Begriff des "focus of attention « im Zusammenhang mit dem Gebrauch des Possessivsuffixes der dritten Person Singular (Fraurud 2001: 260).

Sherwood 2001 (»Definiteness in the Ugrian languages«) thematisiert die Definitheit allgemein in den ugrischen Sprachen, im Fokus stehen dabei der Aspekt des shared $k_{n o w l e d g e}{ }^{55}$ und die diskursrelevanten Eigenschaften der Definitheit (Sherwood 2001: 185). Ausgangspunkt der Analyse ist dabei das als definit zu interpretierende Nomen und die Auswirkungen in der Realisierung der Satzstruktur, zum Beispiel durch die Wahl der objektiven Konjugation. Der Forschungsgegenstand wird am Rande mit dem Hinweis erwähnt, dass das Possessivsuffix der dritten Person Singular und weniger häufig auch das der zweiten Person Singular im Ob-Ugrischen in der Funktion eines postponierten definiten Artikels erscheinen kann (Sherwood 2001: 186).

Auch Ago Künnap fokussiert in seinem Aufsatz "About the non-personal definite function of the Uralic 3rd person possessive suffix « (im Folgenden Künnap 2004) das Possessivsuffix der dritten Person. Er setzt die sekundäre, nicht possessive Funktion gleich mit einem Wegfall der Kodierung der dritten Person und geht dabei von einer grundlegenden Unterscheidung zwischen den Funktionen der Possessivsuffixe der dritten Person gegenüber den anderen beiden Personen aus. Als Argument dient die Ersetzung der ersten und zweiten Personen mit dem Possessivsuffix der dritten Person zum Beispiel in den ostsee-finnischen Sprachen, was umgekehrt nicht der Fall sei. Dieser Unterschied sei nicht ausschließlich eine neuere Entwicklung, sondern darin begründet, dass dem Possessivsuffix der dritten Person generell eine allgemein-definite Funktion inhärent sei:

54 Hier sei auf die ostsee-finnischen Sprachen hingewiesen, in denen eine Tendenz zur Entwicklung und Verwendung von Possessivpronomen zu beobachten ist. Dieser Sprachzweig ist jedoch nicht Gegenstand von Frauruds Analyse, und hier handelt es sich um einen Schwund des Paradigmas der Possessivsuffixe, nicht um einen Funktionswandel.

55 Dieser Begriff entstammt der Pragmatik und kann synonym zu Begriffen wie Präsupposition oder gegebene vs. neue Information gebraucht werden, welche allesamt das gemeinsame Wissen von Sprecher und Hörer bezeichnen (verglichen Gundel/Hinds 1985: 5). 
It [the difference between Px1 and 2 and Px3, G.J.] could go back to the $3 P x$ that was primarily not (only) a possessive suffix but (also) a suffix with some other functions. [...] Thus, it may be supposed that a non-personal general-definite function has always been inherent to the Uralic 3Px. (Künnap 2004: 2f.)

In Bezug auf die ob-ugrischen Sprachen (Ungarisch ist nicht Bestandteil von Künnaps Analyse) hält Künnap 2004 fest, dass sowohl das Possessivsuffix der zweiten als auch der dritten Person in der definiten Funktion auftreten, letzteres jedoch häufiger.

Eine der neuesten Abhandlungen auf diesem Themengebiet ist der Aufsatz »Definitely Not Possessed? Possessive Suffixes with Definiteness Marking Function « von Doris Gerland (im Folgenden Gerland 2014a) ${ }^{56}$ Gerland geht ebenfalls von dem Standpunkt aus, dass die als Markierung von Definitheit beschriebene Funktion eine inhärente Eigenschaft des Possessivsuffixes ist und kein Ergebnis von Grammatikalisierung und attestiert dem Possessivsuffix zwei Funktionen, (a) Erstellung einer Relation zwischen zwei Entitäten und (b) Erstellung einer Relation zwischen einer Entität und dem Diskurs und gleichzeitiger Markierung der Definitheit des Referenten, welcher den Kopf der Konstruktion bildet. Die Interpretation als Possessivmarker (a) oder Definitheitsmarker (b) macht sie dabei vom konzeptuellen Typ des markierten Nomens (»conceptual noun type«, hierzu mehr im Folgenden) und vom Kontext abhängig (Gerland 2014a: 269), das Possessivsuffix sollte demnach eher als relationales Suffix bezeichnet werden:

I will show that instead of a possessive suffix we deal with a relational suffix whose main function is twofold: to indicate a link between two entities, on the one hand, and to mark the entity that bears the suffix as definite, on the other. (Gerland 2014a: 272)

Im ersten, einleitenden Kapitel stellt Gerland die verschiedenen Arten von Possessivkonstruktionen und deren formale Realisierungen sowie die möglichen ausgedrückten Relationen auf Basis von Barker 1995 und Heine 1997 vor. Im Anschluss fasst sie Forschungsstand und Konzept der "anderen Hauptfunktion « der Possessivsuffixe zusammen, nämlich der »Markierung von Definitheit in derselben Art und Weise wie definite Artikel in indo-europäischen Sprachen « (»the possessive suffixes [...] have another main function: they mark definiteness in the same way as definite articles in IndoEuropean languages« Gerland 2014a: 271).

Im folgenden Kapitel stellt Gerland die Definitheitsmarker vor, erst fasst sie die Funktionen und den Gebrauch des definiten Artikels zusammen und stellt dann die Funktionen und den Gebrauch des nicht-possessiven Suffixes als Definitheitsmarker (»Function and Use of the Non-Possessive Suffix as a Definiteness Marker « Gerland 2014a: 274) anhand der Kriterien von Hawkins 1978 vor. Sie kommt zu dem Ergebnis, dass »Possessivsuffixe, wenn sie Definitheit markieren, in allen Verwendungen vorkommen, welche typisch für den definiten Artikel sind « (»the possessive suffixes with definiteness marking function appear in all uses which are assumed typical for definite articles" Gerland 2014a: 274). Gleichzeitig verweist sie aber darauf, dass die Vorkommen von

56 Es existieren zwei weitere, im Internet zugängliche Abhandlungen zu diesem Thema: Gerland 2011 und Gerland 2014b. Die vorliegende Arbeit bezieht sich jedoch nur auf die Publikation Gerland 2014a. 
Possessivsuffixen hier keinesfalls obligatorisch sind, ein Faktor, der allgemein als Hinweis aufgefasst wird, dass sich das Possessivsuffix nicht zum definiten Artikel grammatikalisiert hat (siehe auch Fraurud 2001). Dieser mögliche Grammatikalisierungspfad ist Thema des dritten Abschnittes (Gerland 2014a: 279f.). Hier wird wieder vergleichend der Grammatikalisierungspfad des definiten Artikels mit einem möglichen Grammatikalisierungspfad für das Possessivsuffix in zwei Abschnitten beschrieben, als Ergebnis kann keine Grammatikalisierung zu einem definiten Artikel festgestellt werden:

So far there seems to be no evidence for a grammaticalization path comparable to that of the Indo-European definite articles can be found. (Gerland 2014a: 281)

Die Konsequenz daraus ist laut Gerland, dass beide Funktionen (Markierung von Definitheit und Markierung von possessiven Relationen) dem Possessivsuffix inhärent sind und jeweils der Kontext und die Semantik des suffigierten Nomens die Interpretation begründen, und schlägt daher den Begriff relationales Suffix (»relational suffix» Gerland 2014a: 282) vor.

Der vierte Abschnitt fasst nun die Unterschiede des relationalen Markers je nach possessivem oder nicht-possessivem Gebrauch zusammen (nach Gerland 2014a: 284):

(a) possessive NPn (das heißt mit Possessor)

- Verankerung des Referenten mit einem eindeutigen Referenten;

- Eine mehr oder weniger konkrete Relation (sowohl alienabler als auch inalienabler Art);

- Der markierte Referent ist so semantisch oder pragmatisch eindeutig identifizierbar.

(b) nicht-possessive NPn (das heißt ohne Possessor)

- Verankerung des Referenten durch Assoziation aufgrund von Weltwissen oder der Diskurs-Situation;

- Eine weit gefasste, assoziative Relation jeder Art;

- Der markierte Referent ist so semantisch oder pragmatisch eindeutig identifizierbar.

Demnach bleibt bisweilen die Zweiteilung in eine possessive und eine nicht-possessive Verwendung bestehen, basierend auf der Zuweisung eines möglichen Possessors. Daher ist lediglich eine Umbenennung des Suffixes erfolgt, ohne dass eine tatsächliche ReAnalyse der Verwendungen bislang stattgefunden hätte, die Unterteilung ist im Prinzip dieselbe wie die nach Default-Interpretationen (Teil-Ganzes, Verwandtschaft etc.) als possessiv und Assoziation jeglicher Art (vergleichbar mit der vierten Gruppe nach Aikhenvald/Dixon (Hg.) 2012, siehe unter Kapitel 1.2.2.1) als nicht-possessiv. Im Weiteren befasst sich das Kapitel mit konzeptuellen Typen von Nomen (»conceptual noun types «) und deren Auswirkung auf die Interpretation des relationalen Suffixes (Gerland 2014a: 284). Hintergrund dieser Annahme bildet die Kategorisierung von Nomen in konzeptuelle Typen, zum Beispiel nach Löbner 2011, welche deren grammatischen Gebrauch beeinflussen. Löbner 2011 geht dabei von vier Typen konzeptioneller Nomen aus: »sortal«, "relational «, » functional « und »individual nouns «. »Sortal nouns ( zum Beispiel ,Blume ,Tisch') und »relational nouns« (wie ,Schwester, ,Freund') haben keine Begrenzung an 
möglichen Referenten, auf welche sie sich beziehen können. »Functional nouns« (zum Beispiel ,Mutter', ,President') und »individual nouns « (wie ,Sonne' oder ,Papst') hingegen sind inhärent eindeutig einem Referenten in einem Kontext zuordenbar.

Zusätzlich sind »relational« und »functional nouns « inhärent relational, das heißt, sie brauchen ein weiteres Argument, um zugeordnet werden zu können (verglichen Verwandtschaftstermini), und sind damit vorausbestimmt, um possessiv gebraucht $\mathrm{zu}$ werden; »individual « und "functional nouns « sind aufgrund ihrer Einzigartigkeit vorausbestimmt, definit gebraucht zu werden (Gerland 2014a: 285). Für die Verwendung des Possessivsuffixes ergibt sich demnach eine Skala mit der possessiven Funktion an einer und der definiten Funktion am anderen Ende:

It seems that in possessive NPs with a possessor the relational suffix is interpreted as a possession marker, the definiteness component of the suffix is suspended [...] In definite interpretations the process works the other way round: the possessive component is suspended, the definite interpretation is focused. (Gerland 2014a: 284)

In der Zusammenfassung stellt Gerland fest, dass das Possessivsuffix in uralischen Sprachen auch die Funktion der Definitheitsmarkierung besitzt, diese aber anders als beim definiten Artikel dem Suffix inhärent und nicht Ergebnis einer Grammatikalisierung ist und das Suffix demnach als relationales Suffix bezeichnet werden sollte (Gerland 2014a: 288). Unklar bleibt meines Erachtens jedoch, ob letztlich der konzeptuelle Typ des Nomens oder der Kontext bzw. die Interpretation des Hörers der ausschlaggebende Faktor für die Interpretation possessiv oder definit ist, da zum einen auf die Rolle des Kontextes unterschiedlich verwiesen wird:

It is the context and the semantics of the respective noun which trigger the interpretation as a definiteness marker or a possession marker. (Gerland 2014a: 282)

versus

The interpretation of the relational suffix as a marker of possession or definiteness is depends on the semantics of the marked noun and the context is secondary. (Gerland 2014a: 284)

und

The interpretation, whether the suffix represents a possessive relation or an associative relation, is up to the hearer. (Gerland 2014a: 284)

Andererseits scheinen je nach konzeptuellem Typ beide Interpretationen bzw. auch ein Wechsel des Typs (»type shift« Gerland 2014a: 287f.) - je nach Kontext - möglich:

Functional nouns such as father and boss are both inherently unique and inherently relational. [...] Hence a functional noun such as father requires a definite and a possessive construction for disambiguation, which in these cases is combined in the relational suffix. Consequently both readings, the possessive and the definite, lead to the same referent. (Gerland 2014a: 286)

Der Forschungsansatz der Definitheits-Theorie geht aus der Tatsache hervor, dass die Ansichten und Ergebnisse zu Possessivkonstruktionen, wie sie basierend auf den 
Einsichten aus den indo-europäischen Sprachen erfolgt sind, nicht vergleichbar mit denen der uralischen Sprachen sind, sowohl in der formalen Markierung als auch im Vorkommen bzw. der Verwendung. Vom typologischen Standpunkt aus ist es naheliegend, einen Erklärungsansatz für die Possessivkonstruktionen des Uralischen heranzuziehen, welcher Berührungspunkte mit dem der Possession hat. Die Interpretation des Possessivsuffixes als Definitheitsmarker ist dafür insofern geeignet, als beide Mechanismen im selben Bereich verankert sind (der Identifizierbarkeit eines Referenten mithilfe des Kontextes, Details dazu siehe Kapitel 3.4) und das Possessivsuffix formal gewisse Ähnlichkeiten mit dem definiten Artikel aufweist: (i) eine Entwicklung aus einer demonstrativ-deiktischen Pro-Form, (ii) ein nominaler Modifizierer, (iii) das Vorkommen in einer NP zusammen mit dem zu determinierenden Referenten.

Problematisch ist meines Erachtens dabei jedoch der auf der Annahme eines möglichen Possessors basierende Argumentationsverlauf und die daraus resultierende Zweiteilung von possessiver und nicht-possessiver, prototypischer und nicht-prototypischer bzw. primärer und sekundärer Funktion des Possessivsuffixes. Diese ist meines Erachtens einzig und allein sozio-kulturellen Faktoren geschuldet, welche für bestimmte Wortklassen keine Interpretation eines Possessors im engeren Sinne zulassen. Ein weiteres Problem der Definitheits-Theorie ist meines Erachtens die Fokussierung auf den Kopf der Konstruktion: Durch den Wegfall eines möglichen Possessors und den Vergleich mit anderen Determinierern, welche ausschließlich an einer Position den Kopf der Konstruktion modifizieren, sind die zugrunde liegenden Faktoren, welche die Realisierung des Modifizierers als Leerstelle betreffen, nicht mehr Gegenstand der Analyse. Insbesondere beim Vergleich mit dem definiten Artikel rückt zusätzlich die Berücksichtigung der im Suffix kodierten Person in den Hintergrund. Die meisten Abhandlungen belassen es bei einem Hinweis auf weitere mögliche Possessivsuffixe in der sekundären Funktion, bauen die Analyse jedoch auf das Possessivsuffix der dritten Person Singular auf.

\subsubsection{Neuere Forschungsansätze nach der Definitheit}

Auch der Ansatz der Definitheits-Theorie vermag somit keine zufriedenstellende und letztliche Erklärung für sämtliche Verwendungen der Possessivkonstruktionen in den uralischen Sprachen, welche nicht dem prototypischen Ansatz zur Possession entsprechen, zu liefern. Somit haben sich in jüngerer Zeit weitere Forschungsansätze entwickelt, welche dabei neuere linguistische Teildisziplinen und daraus gewonnene Erkenntnisse mit in die Analyse einbeziehen. Zum einem handelt es sich dabei um Forschungen, welche einen kognitiv-linguistischen Ansatz verfolgen, zum anderen pragmatisch-informations-strukturelle Ansätze (Details zu beiden Theorien siehe Kapitel 3.1 und 3.2).

Die Analyse der Possessivkonstruktionen innerhalb der Theorie der ReferenzpunktKonstruktion ist zu einem der Beschreibungsansätze zur Possession generell geworden, insbesondere die Werke von Langacker 1993 und Langacker 1995 sind hier zu erwähnen. Ebenso basiert die Monografie von Taylor 1996 zur Possession im Englischen auf dem kognitiven Ansatz, gleichfalls Heine 1997. Eine der neuesten Publikationen in diesem Bereich ist der Sammelband von McGregor (Hg.) 2009, welcher nicht nur den 
Ansatz der Referenzpunkt-Konstruktion auf das Englische thematisiert, sondern durch Miteinbezug der Textumgebung die Beobachtungen von Taylor für das Englische widerlegt (Willemse 2009: 14).

In der Uralistik hat Gábor Tolcsvai Nagy 2004 die Possessivkonstruktion des Ungarischen im Rahmen der Referenzpunkt-Konstruktion beschrieben, und auch die Grammatik von Irina Nikolaeva 1999a zum Obdorsk-Chantischen bezieht den kognitiven Ansatz mit ein.

In ihrer Grammatik »Ostyak « (Nikolaeva 1999a) verfolgt Nikolaeva zunächst ebenfalls den formalen Ansatz: Nach der Beschreibung des Paradigmas im Bereich der nominalen Morphologie widmet sich das Kapitel »Syntax « unter dem Punkt »Possession « einer Beschreibung von possessiven und nicht-possessiven Nominalphrasen (Nikolaeva 1999a: 51f.). Diese Unterteilung erfolgt jedoch nicht nach konstruktionsbedingten Kriterien wie etwa der Realisierung des Modifizierers, sondern primär semantisch: Beide Typen der adnominalen Possessivkonstruktion, mit Possessivsuffix und unmarkiert, werden gemeinsam beschrieben, ausgehend von ihrer Lesart als Possession. Der zweite Unterpunkt befasst sich mit allen anderen Typen von Nominalphrasen, das heißt, auch hier werden neben Adjektiven und Numeralia als Attribute ebenfalls Konstruktionen vom Typ Nomen Nomen subsumiert, welche sich formal nicht von denen bei den possessiven Nominalphrasen unterscheiden. Auf die semantisch basierten Kriterien dieser Unterteilung wird an dieser Stelle nicht weiter eingegangen (Nikolaeva 1999a: 52). Diese werden stattdessen im zweiten Teil im Kapitel der Relativsätze noch einmal aufgegriffen. Nikolaeva bezieht sich bei der Analyse der Semantik der Possessivkonstruktionen auf Seiler 1973 und Barker 1995 und wendet Barkers Ansatz auf das Chantische an (Nikolaeva 1999a: 81) - meines Wissens die erste und einzige Abhandlung dieser Art zum Konzept Possession in der Uralistik.

Darüber hinaus berücksichtigt sie den kognitiven Ansatz von Langacker 1993 und 1995 und vergleicht das Vorkommen der chantischen Possessivsuffixe mit den Taylorschen Ergebnissen ${ }^{57}$ (Nikolaeva 1999a: 80f.) und verweist außerdem auf die Rolle des Kontextes (Nikolaeva 1999a: 51):

Apparent ambiguity of possessives has led some scholars to propose that the possessive construction is semantically indeterminate and can express any kind of relationship between two entities [...] Kay and Zimmer (1976) argue that the possessive (in their terms, Genitive) NP is simply a metalinguistic instruction to the hearer that there is some kind of relation between the modified and the modifier. The hearer supplies an appropriate interpretation within a given context. (Nikolaeva 1999a: 80f.)

Ihre Analyse kommt zu dem Ergebnis, dass anders als zum Beispiel in den indo-europäischen Sprachen wie bei Taylor 1996 am Beispiel des Englischen beschrieben Possessivkonstruktionen im Chantischen generell häufiger auftreten und öfter eine Assoziation ausdrücken als eine der Default-Interpretationen:

For comparison, the analysis of three Ostyak folklore texts from a total length of 3,500 words has revealed 270 occurrences of possessive NPs, that is, every 
thirteenth word functions as the possessor within the possessive construction. Possessives, thus, are four times more frequent in Ostyak than in English. Furthermore, in Taylor's corpus only $16 \%$ of possessives have an associative meaning, see Taylor (1996: 346-347). In Ostyak this percentage is as high as 39\% for the corpus analyzed. (Nikolaeva 1999a: 82)

Zudem weist sie auf die Optionalität der Verwendung von Possessivkonstruktionen hin, das Weglassen des Possessivsuffixes führt nicht automatisch zu einem un-grammatikalischen Satz. Sie beschreibt die Verwendung der Possessivsuffixe daher als Mittel des Sprechers, eine Relation hervorzuheben und attestiert der Verwendung der Possessivkonstruktion damit einen pragmatischen Beweggrund (bei ihr wird diese Verwendung als »pragmatic associative possessives« bezeichnet Nikolaeva 1999a: 82).

Die Berücksichtigung der Textumgebung und den damit verbundenen kognitivpragmatischen Aspekten hat generell in neueren Grammatiken zu detaillierteren Beschreibungen von pronominalen Markern geführt, unter anderem auch in Bezug auf die Realisierung des Modifizierers in der attributiven Possessivkonstruktion. UllaMaija Kulonen stellt 2007 beispielsweise in ihrer Grammatik des Ost-Mansischen (Kulonen 2007) einen deutlichen Zusammenhang von pronominalen Possessoren und Possessivsuffix her, welcher so detailliert in den früheren Handbüchern nicht zu finden ist, zum Beispiel:

Kun omistaja on tunnettu riittää pelkkä omistetun possessiivisuffiksi. [...] Persoonapronomini omistajana pannaan lauseessa ilmi vain tarvittaessa: silloin kun omistajaa halutaan korostaa tai jos se selvyyden vuoksi halutaan panna näkyviin [...]. Harvoin voidaan pronominiomistajan läsnä ollessa jättää omistetun possessiivisuffiksi pois. (Ist der Besitzer bekannt, reicht das Possessivsuffix als Marker aus. [...] Der Besitzer wird nur als Personalpronomen realisiert, wenn nötig: dann wenn der Besitzer betont werden soll oder man ihn aus Gründen der Disambiguierung erwähnen möchte. [...] Selten ist es möglich, bei einem pronominal erwähnten Possessor das Possessivsuffix weg zu lassen). (Kulonen 2007: 195)

Kulonen 2007 unterscheidet sich außerdem im Aufbau von Beschreibungen zum Possessivsuffix anderer Grammatiken. Sie liefert zum einen im Abschnitt »Morphologie» eine detaillierte morpho(phono)logische Analyse der Possessivsuffixe (die Beschreibung des Paradigmas erstreckt sich über neun Seiten, auf denen sämtliche allomorphe Formen für das gesamte $33^{\star} 3$ Formen umfassende Paradigma gelistet werden, verglichen Kulonen 2007: 31-45). Zu Beginn wird dabei kurz auf die Funktionen von Possessivsuffixen hingewiesen, die »eng mit dem Ausdruck von Possession, sonstiger enger Verbindung und Teil-Ganzes-Relationen verbunden sind « (»Possessiivisuffiksien funktiot liityvät läheisesti omistamisen ja muun läheisen yhteenkuuluvuuden sekä osa- kokonaisuussuhteen ilmaiseniseen « Kulonen 2007: 32). Diesem Abschnitt folgt im Kapitel »Morphologie« der Punkt »Kasusbildung und Kasusgebrauch« (»Sijataivutus ja sijamuotojen funktiot « Kulonen 2007: 27-53). Hier unterteilt Kulonen 2007 die nominale Deklination in eine absolute und eine possessive und führt in dieser Konsequenz für jedes Kasussuffix sowohl die absoluten (das heißt der betreffende Kasus in Singular, Dual und Plural) als auch die possessiven Formen (das heißt sämtliche in den im Anhang befindlichen Texten belegte Formen von Px-Cx) an. Diese Paradigmen bezeichnet sie jeweils beispielsweise als »Possessiver Akkusativ«, »Possessiver Lokativ«, »Possessiver 
Instrumental« usw. (»possessiivinen akkusatiivi« etc., verglichen Kulonen 2007: 52, 65f., 77), was meines Erachtens in der Hinsicht problematisch ist, dass somit besondere Kasusformen für die possessive Deklination suggeriert werden, jedoch lediglich die Reihenfolge Px-Cx gemeint ist. Darüber hinaus ist den Possessivkonstruktionen ein eigener Abschnitt im Kapitel »Syntax« gewidmet, in welchem adnominale, lokale und prädikative Possessivkonstruktionen behandelt werden (Kulonen 2007: 195f.).

Insbesondere der Ansatz der Pragmatik hat im Bereich der Uralistik in vielerlei Hinsicht zu neuen Forschungsansätzen und der Re-Evaluierung vieler bis dahin sich an der Schulgrammatik bzw. an der traditionellen indo-europäischen und lateinischen Grammatikschreibung orientierten Ansichten veranlasst. So hat zum Beispiel die Forschung zum Passiv eine völlig neue Analyserichtung mit Einbezug der Pragmatik genommen.

Aber auch aufgrund der Tatsache, dass die uralischen Sprachen als sogenannte pro-drop-Sprachen nicht nur mit einer Bandbreite von pronominalen Markern, sondern auch mit sogenannten Nullmorphemen arbeiten, ist die Pragmatik - insbesondere im Bereich der Topik-Kontinuität - in der Uralistik von hohem Wert. Im Zusammenhang mit den Possessivsuffixen gibt es neuere Erkenntnisse, welche in den folgenden Jahren und Jahrzehnten in Aufsätze und Grammatiken mit eingeflossen sind.

Die »Grammatik des Ostchantischen« (»Eastern Ostyak Grammar», im Folgenden Filchenko 2007) gibt nach der formalen Übersicht zum Paradigma der Possessivsuffixe (Filchenko 2007: 80f.) einen knappen Abriss zur Lesart des Konzepts Possession, weist aber auf eine detaillierte Analyse der Funktionen des Possessivsuffixes im Kapitel zu Pragmatik und Textkohäsion hin (Filchenko 2007: 81).

Der fehlende Verweis, um welches Kapitel es sich letztlich handelt (es existiert beispielsweise ein Kapitel »10.2 Information Structure «, Seiten 373-389, in welchem jedoch die Possessivsuffixe nicht als zentrales Thema behandelt werden, sondern zum Beispiel die pragmatischen Rollen Topic und Fokus beschrieben werden. Ein Abschnitt mit der Überschrift Pragmatik, Textkohäsion oder dergleichen existiert hingegen nicht), erschwert eine umfassende Beschreibung der Darstellung der Possessivsuffixe im Forschungsstand der vorliegenden Arbeit. So kann nur auf weitere Abschnitte in Filchenko 2007 hingewiesen werden, in welchen Possessivsuffixe und deren pragmatische Eigenschaften thematisiert werden. So werden beispielsweise im Punkt »9.2.1 Bound modifiers « Possessivsuffixe unter den Determinierern erwähnt (Filchenko 2007: 294), in Abschnitt "9.2.2 Lexical word-size modifiers « wird auf possessive Determinierer wie ,mein', ,dein' verwiesen, womit die Realisierung des Modifizierers als Personalpronomen gemeint ist. Es wird auf zwei unterschiedliche Konstruktionsarten verwiesen, mit Possessivsuffix und mit Personalpronomen, ein Hinweis, dass letzteres zusätzlich zum Suffix erscheint, fehlt und muss aus dem zugehörigen Beispiel erschlossen werden (Filchenko 2007: 297). Erwähnenswert ist der Hinweis zur Kombination von Demonstrativpronomen und Possessivsuffix, wo laut Filchenko der Konstruktionstyp 1.b.3 häufiger erscheint als 1.b.2 (»zusätzliche pronominale Realisierung«) (Filchenko 2007: 297).

Zusätzlich ist seit Mitte der 1990er-Jahre eine Bandbreite von Aufsätzen entstanden, welche sich mehr und mehr in Richtung der Pragmatik und Informations-Struktur orientieren. Hier wird auch auf die Zusammenhänge von Pragmatik und Verwendung des Possessivsuffixes verwiesen, jedoch oftmals der Vollständigkeit halber; im Fokus stehen andere pragmatisch motivierte Erscheinungen, eine Beschreibung der Possessivsuffixe 
in dieser Hinsicht fehlt bislang. So wird beispielsweise auch auf die Rolle der Possessivsuffixe im Zusammenhang mit den »kommunikativen Rollen « - wie die pragmatischen Rollen 1993 noch bei Nikolaeva/Kovgan/Koškarëva 1993 genannt werden - bis hin zu Skribnik 2001 hingewiesen. Das sogenannte reference-tracking, das Verfolgen eines Partizipanten in einem fortlaufenden Diskurs (Nagaya 2006: 3), befasst sich mit der Verwendung der in einer Sprache zur Verfügung stehenden anaphorischen Verweismittel in Relation zur Topikalität des jeweiligen Referenten. Kovgan 2001 und Kovgan 2005 verweisen bei ihrer Analyse des Chantischen auch in einem Abschnitt auf Possessivsuffixe in diesem Zusammenhang.

Irina Nikolaeva greift in ihrem Aufsatz »Possessive affixes in the pragmatic structuring of the utterance: Evidence from Uralic « (im weiteren Nikolaeva 2003) den Ansatz der Definitheits-Theorie auf und eröffnet ausgehend von denjenigen Aspekten der Possessivsuffixe, welche nicht mit dem Ansatz der Definitheits-Theorie erklärt werden können, einen neuen Analyseansatz zum nicht-possessiven Gebrauch der Possessivsuffixe mit Zuhilfenahme pragmatischer Ansätze zusammen mit einem Ausblick auf den Ansatz der Kognitiven Linguistik.

Ihr Aufsatz stellt zunächst die bislang angenommene Distinktion zwischen dem possessiven und dem nicht-possessiven Gebrauch der Possessivsuffixe dar, wobei sie ebenfalls die Distinktion auf Basis der Semantik des Kopfes begründet anhand von Beispielen aus mehreren uralischen Sprachen, darunter auch Nord-Chantisch. Ein zentrales Argument ist dabei die Verwendung aller Personen und Numeri, was bei bisherigen Abhandlungen zugunsten der Beschreibung der dritten Person Singular kaum berücksichtigt wurde (Nikolaeva 2003: 1f.). Im zweiten Abschnitt konzentriert sie sich stattdessen auf den Aspekt der Identifizierbarkeit (siehe dazu Kapitel 3), der sowohl definiten Artikeln als auch Possessivsuffixen zugrunde liegt, jedoch nicht synonym zu Definitheit ist und von den Possessivsuffixen im Uralischen nicht im dem Umfang ausgedrückt werde, dass es sich dabei um den Ausdruck der grammatischen Kategorie Definitheit handelte oder eine Grammatikalisierung zum definiten Artikel zu belegen wäre (Nikolaeva 2003: 2-5). Sie stellt zwei Arten der Identifizierbarkeit vor, welche auch und ausschließlich mittels des Possessivsuffixes der dritten Person Singular ausgedrückt werden können: (a) Identifizierbarkeit basierend auf Deixis und situationsbedingtem Kontext (»identifiability based on deixis and situational context«) und (b) Identifizierbarkeit basierend auf Anaphorik (»identifiability based on anaphora «).

Identifizierbarkeit, welche auf Deixis beruht, bezieht sich auf Referenten, die dadurch in einer bestimmten Situation identifizierbar sind, da sie sichtbar oder in anderer Weise salient sind, mit anderen Worten, ein konkreter Teil der Äußerungssituation sind und damit alle Sprechaktteilnehmer eindeutig den betreffenden Referenten identifizieren können. Hierunter fallen laut Nikolaeva 2003 auch Possessivkonstruktionen mit Substantiven, welche Objekte oder Phänomene aus der Natur beschreiben, zum Beispiel ,Regen' oder ,Gras' (verglichen Nikolaeva 2003: 3). Die situationsbedingte Identifizierbarkeit beinhaltet sowohl Identifizierbarkeit aufgrund des »larger situational context « - wie bei ,Sonne‘, ,Mond - als auch den »restricted situational context «, beispielsweise bei ,Morgen', ,Sommer' (Nikolaeva 2003: 3).

In der zweiten Kategorie - Identifizierbarkeit basierend auf Anaphorik - stellt Nikolaeva 2003 Beispiele vor, in welchen zuvor eingeführte Referenten im Folgesatz erneut 
aufgegriffen werden, und zwar in Form einer Nominalphrase mit Possessivsuffix. Dieser Mechanismus scheint in den uralischen Sprachen häufiger aufzutreten (verglichen Belege aus dem Komi, Selkupischen und Chantischen bei Nikolaeva 2003) und wird auch in der vorliegenden Arbeit in Kapitel 5.2.1.2 näher beschrieben. Der dritte Abschnitt (Nikolaeva 2003: 5-10) beschäftigt sich dann mit der grundlegenden Funktion der Possessivkonstruktion, eine Relation zwischen zwei Entitäten auszudrücken. Diese Relation ist prinzipiell semantisch unbestimmt (»semantically indeterminate« Nikolaeva 2003: 6), die Possessivkonstruktion wird daher nach Kay/Zimmer 1976 (dort resp. Genitiv-Konstruktion) als »metalinguistische Anleitung für den Hörer, dass eine Relation zwischen Genitiv und Kopf besteht « (»metalinguistic instruction to the hearer that there is some kind of relation between the Genitive and the head « Nikolaeva 2003: 6) beschrieben. Erfolgt die Interpretation der Relation dann durch den Kontext bzw. die Äußerungssituation sowie der darin zugrunde liegenden Intention des Sprechers, handelt es sich laut Nikolaeva 2003 um eine inhaltsorientierte Relation (»associative relationship« Nikolaeva 2003: 6). Letztlich verweist Nikolaeva 2003 demnach sowohl auf die informations-strukturellen als auch die text-strukturierenden Eigenschaften der Possessivsuffixe. In diesem Abschnitt werden ebenfalls zwei unterschiedliche Verwendungsarten von Possessivkonstruktionen vorgestellt, welche den Kontext mit einbeziehen: (a) Verknüpfung mit dem Sprechakt (»linking to the speech setting «, Nikolaeva 2003: 6f.) und (b) Verknüpfung mit einem Element innerhalb des Diskurses (»linking to a discourse-internal element ", Nikolaeva 2003: 8f.).

Bei der Verknüpfung mit dem Sprechakt referieren die Possessivsuffixe der ersten und zweiten Person auf Entitäten, die in der außersprachlichen Welt, das heißt im Sprechakt, salient sind - sprich auf die Sprechaktteilnehmer. Im Dialog erfolgt somit die Identifizierung eines Referenten durch die Verknüpfung mittels Possessivsuffix mit einem der Sprechaktteilnehmer (Nikolaeva 2003: 6). Je nach Verknüpfung mit Sprecher oder Hörer werden hier unterschiedliche Effekte erzielt: Laut Nikolaeva 2003 wird die erste Person genutzt, um eine enge pragmatische Relation zwischen Sprecher und Adressat (in diesem Falle gleichzeitig auch der Hörer) zu erstellen. Als Beispiele gibt sie Äußerungen an, die zum Beispiel dem deutschen ,Meine Damen und Herren ‘̈ ähnlich sind (Nikolaeva 2003: 6).

Wird das Possessivsuffix der zweiten Person verwendet, so entsteht laut Nikolaeva 2003 eine vom Sprecher indizierte pragmatische Assoziation zwischen dem Hörer und dem Referenten des Nomens, welches das Possessivsuffix trägt. Oftmals sind solche Belege in Imperativsätzen zu finden. Der Abschnitt verweist abschließend auf die Optionalität des Possessivsuffixes in diesem Zusammenhang, welche einzig und allein von der Intention des Sprechers abhängt.

Dabei wird eine semantische Assoziation zwischen zwei Entitäten erstellt, deren Interpretation maßgeblich von Situation und Kontext abhängt. Alle Personen könnten auf diese Art verwendet werden (Nikolaeva 203: 10).

Im vorletzten Abschnitt (Nikolaeva 2003: 11f.) beschreibt sie eine Art der Fokussierung mittels Possessivsuffix dritte Person Singular in den uralischen Sprachen im Wolga-Kama-Areal, auf welche in Kapitel 5.2.1.2 und 5.2.1.3 noch einmal eingegangen wird. Anhand der Beispiele von Nikolaeva 2003 geht hervor, dass die Struktur hier wie folgt ist: Entweder werden zwei oder mehrere Referenten im ersten Satz eingeführt und 
im Folgesatz jeweils einer davon wieder aufgegriffen, dieser wird dabei mit dem Possessivsuffix der dritten Person Singular markiert. Variante zwei, die Information des ersten Satzes wird im Folgesatz mit einem neuen, kontrastiven Referenten verknüpft, dieser wird mit Possessivsuffix der dritten Person Singular versehen. In beiden Fällen sind die Alternativen, aus denen eine fokussiert und herausgegriffen wird, unmittelbar vorerwähnt. Ein Parameter, diese Funktion zu beschreiben, besteht laut Nikolaeva 2003 in der Tatsache, dass hier die Voraussetzung der Identifizierbarkeit nicht gegeben sein muss:

Identifiability has to do with the ability of interlocutors to pick up a referent unambiguously, while contrast/emphasis pertains to the relative saliency of the respective concept for the speaker. (Nikolaeva 2003: 11)

Somit ist die Fokussierung nicht an die Wortart Substantiv gebunden, sondern auch Adverbien können Possessivsuffixe mit dieser Funktion tragen.

In der anschließenden Zusammenfassung ihres Aufsatzes setzt Nikolaeva 2003 die grundlegende Funktion der Possessivkonstruktionen, eine Relation bzw. Assoziation zwischen zwei Entitäten anzuzeigen, mit dem Ansatz der Referenzpunkt-Konstruktionen der kognitiven Linguistik in Relation (siehe dazu auch Kapitel 3.1). Dabei unterteilt sie die Possessivkonstruktionen in zwei Gruppen: in solche mit sogenannten relationalen Nomen, welche prototypische Referenzpunkt-Konstruktionen repräsentieren, und in solche, deren prinzipiell uneingeschränkte Interpretation der Assoziation vom Kontext abhängt. Letztere sind diejenigen Possessivkonstruktionen, welche im Gegensatz zu anderen Sprachfamilien im Uralischen nicht nur möglich sind, sondern auffallend häufig gebraucht werden (die sogenannten nicht-possessiven Possessivkonstruktionen). Voraussetzung für die Verwendung ist dabei lediglich die Vorerwähntheit bzw. Etablierung des Referenten im Diskurs bzw. Kontext, der mit dem Possessivsuffix kodiert - vom kognitiven Gesichtspunkt aus, der Referenzpunkt der Konstruktion und damit wiederum identifizierbar ist.

Über die Assoziation mit diesem Referenzpunkt wird auch der Kopf der Konstruktion (bei Nikolaeva das " possessed noun « Nikolaeva 2003: 13) identifizierbar. Nikolaeva 2003 beschreibt danach im letzten Abschnitt den zugrunde liegenden theoretischen Ansatz der kognitiven Referenzpunkt-Konstruktion und weist auf Arbeiten in der Uralistik hin, welche diesem Ansatz in einer generalisierten Form bei der Beschreibung von Possessivsuffixen folgen, hin. Wenn auch in diesem Aufsatz die Zweiteilung in eine possessive und eine nicht-possessive Funktion des Possessivsuffixes als Grundlage der Analyse weitestgehend bestehen bleibt, setzt sich Nikolaeva 2003 doch kritisch mit dem Definitheits-Ansatz auseinander und stellt einen alternativen Analyseansatz vor, welcher einen Aspekt fokussiert, der bei beiden Funktionen in gewisser Weise zugrunde liegt: die sogenannte Identifizierbarkeit. Zusätzlich greift sie Aspekte der Pragmatik und kognitiven Linguistik auf, welche auch die Grundlage der vorliegenden Arbeit bilden. Nikolaevas Beobachtungen werden daher im empirischen Teil der vorliegenden Arbeit Berücksichtigung finden.

In seinem Aufsatz »Possessive constructions in Eastern Khanty« (im Folgenden Filchenko 2014) beschreibt Andrey Filchenko die grundlegenden morpho-syntaktischen und semantischen Eigenschaften der Possessivkonstruktionen im Ost-Chantischen anhand dreier Dialekte dieser Gruppe (Vach-Vasjugan, Alexandrovo). Die Daten, welche 
Teil des Tomsker Archivs sind, enthalten unter anderem auch Erzählungen jüngeren Datums (2005-2013).

Ziel des Aufsatzes ist es, die bisherigen semantisch- und/oder morpho-syntaktisch basierten Forschungsansätze zur Possession in der Uralistik auf die Daten des OstChantischen anzuwenden. Einleitend referiert Filchenko 2014 kurz auf die bisherigen Forschungsansätze und skizziert die Unterteilung der Possessivkonstruktionen anhand ihrer morphosyntaktischen Eigenschaften in adnominale und prädikative Konstruktionen und verweist auch auf die externe Possession als weiteres Beschreibungskriterium (verglichen Filchenko 2014: 83).

Analog zu dieser Unterteilung sind die folgenden zwei Kapitel aufgebaut, sodass zunächst die adnominalen und im Folgekapitel die prädikativen Possessivkonstruktionen behandelt werden. Bei den adnominalen Konstruktionen geht er als Grundkonstruktion von der unmarkierten Juxtaposition aus und verweist darauf, dass es im Ost-Chantischen keine Kasusmarkierung vergleichbar mit dem Genitiv in anderen Sprachen gibt, dann folgen Beispiele mit Possessivsuffixen. Das nächste Set von Beispielen bezieht sich auf die Realisierung des Modifizierers als Personalpronomen, jedoch steht die Realisierung und nicht die Markierung des Kopfes in Fokus. Die Zusammenstellung der Beispiele soll verdeutlichen, dass der Possessor sowohl als Personalpronomen als auch als Substantiv kein dependent-marking erhält, auf die Possessivsuffigierung am Kopf wird dabei nicht verwiesen.

Das letzte Set von Beispielen enthält wieder Possessivsuffixe, dieses Mal wird darauf hingewiesen, dass »die possessive Relation nicht explizit kodiert werden muss und eher durch den Kontext aufgrund vorhandener Topikalität impliziert und rückbezogen werden kann« (»demonstrates that the possessive relation may be not coded explicitly at all and is rather implicit and recoverable from the context as highly topical « Filchenko 2014: 85). Die formale Beschreibung der Konstruktionstypen endet mit einem Konstruktionsschema, danach folgt eine semantische Analyse, mit dem Hinweis, dass die bisherigen Beispiele alienable Possessivrelationen repräsentieren. Danach werden Beispiele vorgestellt, die inalienable Possession ausdrücken, zum Beispiel Körperteile und Verwandtschaftsverhältnisse, die im Ost-Chantischen laut Filchenko 2014 nicht ohne Possessivsuffix auftreten können (verglichen Filchenko 2014: 84f.).

Der folgende Abschnitt befasst sich dann mit der prädikativen Possession: Hier werden zunächst verschiedene Arten von Existential- bzw. Lokalsätzen und anschließend transitive Habeo-Konstruktionen vorgestellt (verglichen Filchenko 2014: 85f.).

Mit Kapitel Vier (Filchenko 2014: 88f.) wird der bisherige Aufbau des Aufsatzes - die Systematisierung und Beschreibung von Konstruktionsarten zum Ausdruck possessiver Relationen - unterbrochen, denn nun rückt das Possessivsuffix, auf welches bislang nur am Rande verwiesen wurde, in seiner Funktion als pragmatischer Marker in den Fokus. Das Kapitel beginnt jedoch nicht mit der Beschreibung der Possessivsuffixe, sondern einer kurzen Einführung in die Informations-Strukturierung im Ost-Chantischen Satz in Relation zur objektiven Konjugation - somit fehlt zum Beispiel eine grundlegende Beschreibung des Paradigmas der Possessivsuffixe sowie deren Kodierung und Ähnliches.

Über Reflexivität und objektive Konjugation wird dann ein Bogen geschlagen zur Zuordnung des Referenten in einem transitiven Satz, in dem Subjekt und direktes 
Objekt sowohl lexikalisch erwähnt als auch am Verb kodiert sind (mittels der objektiven Konjugation, OK). Zusammengefasst kodieren nominale (Possessivsuffixe) und verbale Personalmarker (OK) aufgrund ihrer informations-strukturellen Eigenschaften solche Antezendenten, welche zum Beispiel auch die Reflexivität im Satz kontrollieren (Filchenko 2014: 89).

Auf diesen Abschnitt folgt eine kurze Beschreibung der Subordination mittels infiniter Verbalkonstruktionen (Relativsätze) und der Markierung des Kopfes mit Possessivsuffixen, welche denselben informations-strukturellen Bedingungen unterliegt wie die oben beschriebene OK und die Reflexivität. Das Kapitel endet mit einer Zusammenfassung der informations-strukturellen Eigenschaften der Possessivsuffixe:

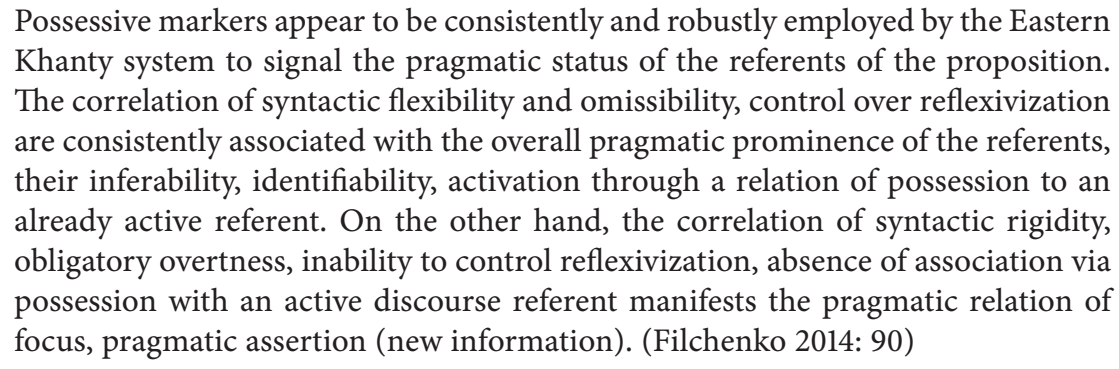

Das fünfte Kapitel (Filchenko 2014: 90f.) befasst sich mit dem Proprietiv ${ }^{58}$ und stellt einen Vergleich zwischen Derivationssuffixen und Adverbialkasus und der Dimension Possession an und greift somit das Thema der Kapitel Eins bis Drei wieder auf, wohingegen Kapitel Vier einen völlig anderen thematischen Schwerpunkt hat.

Kapitel Sechs (Filchenko 2014: 92f.) widmet sich der externen Possession. Die folgenden Beispiele zeigen mögliche Konstruktionen, welche laut Definition nach Payne/ Barshi (Hg.) 1999 als externe Possession angesehen werden können; die Analyse als solche wird jedoch zur Diskussion gestellt (Filchenko 2014: 92), verglichen auch Kapitel 1.2.2.1. Auf die Markierung mit Possessivsuffix wird auch in diesem Kapitel nicht weiter eingegangen.

In der Zusammenfassung (Filchenko 2014: 93) werden noch einmal die verschiedenen Konstruktionstypen aufgezählt, mit welchen possessive Relationen im Ost-Chantischen ausgedrückt werden können. Als die häufigsten Konstruktionen werden die adnominalen sowie die lokalen Habeo-Konstruktionen angesehen, wohingegen proprietive Konstruktionen sowie die Verwendung von Possessivsuffixen eher auf einer lexikal-morphologischen Ebene Gebrauch finden. Des Weiteren wird auf die Kodierung topikaler Referenten mittels Possessivsuffixe hingewiesen, womit auf der syntaktischpragmatischen Ebene Kohärenz im Diskurs erstellt wird und die Informations-Strukturierung erfolgt (Filchenko 2014: 93).

Der Aufsatz hat zwei Schwerpunkte: Zum einen enthält er eine vollständige onomasiologische Zusammenfassung sämtlicher Konstruktionsarten, welche im heutigen OstChantischen zum Ausdruck von Possession vorkommen können. Zusätzlich gibt der 
Aufsatz einen guten Einblick in den Bereich der externen Possession und die Anwendung der Theorie auf eine uralische Sprache. Die vorliegende Arbeit folgt insofern in diesem Aspekt Filchenko 2014, als die für nicht-uralische Sprachen ausgearbeiteten Ansätze zur externen Possession bislang nicht ausreichend an uralischen Sprachen erforscht wurden und daher nicht in der Analyse berücksichtigt werden; des Weiteren wird die syntaktische Terminologie zur Beschreibung der Possessivkonstruktion - Modifizierer und Kopf - auch in der vorliegenden Arbeit verwendet.

Der zweite Aspekt des Aufsatzes ist die informations-strukturierende Rolle der Possessivsuffixe im Diskurs. Diese wird bei Filchenko 2014 auf Grundlage von zusammenhängenden Texten analysiert sowie auf den Zusammenhang zwischen Pragmatik und Possessivmarkierung hingewiesen und berücksichtigt somit einen Forschungsansatz, welchen auch die vorliegende Arbeit verfolgt. Leider beschränkt sich die Beschreibung der Ergebnisse auf ein einziges Kapitel innerhalb der onomasiologisch-basierten Beschreibung von Possessivkonstruktionen im Ost-Chantischen und kann somit nicht detailliert genug ausfallen, um als Grundlage für die vorliegende Arbeit herangezogen werden zu können. Meines Erachtens wäre man diesem Aspekt in einer eigenen Abhandlung gerechter geworden, der Erkenntnisgewinn wird jedoch berücksichtigt.

In ihrem Aufsatz »Microvariation in Finno-Ugric possessive markers« (im Folgenden Simonenko 2014) befasst sich Alexandra Simonenko mit dem Gebrauch von Possessivsuffixen in nicht-possessiven Kontexten in drei finno-ugrischen Sprachen (Komi, der Šuryškary Dialekt des Nord-Chantischen und Mari). Das Hauptaugenmerk der Arbeit liegt dabei auf den Gebrauchsunterschieden zwischen den drei behandelten Sprachen.

Der Ansatz des synchronen Vergleichs ist eine Annahme der Variationen als Ausdruck eines referentiellen Markers (»reference-related marker «, Simonenko 2014: 1). Vorgestellt wird die Analyse anhand des Possessivsuffixes der dritten Person Singular. Abschließend soll auch gezeigt werden, dass sowohl dem possessiven als auch dem sogenannten nicht-possessiven Gebrauch dieselben semantischen Mechanismen zugrunde liegen, und zwar auf Basis der Annahme, dass die Salienz des Referenten, welcher den Kopf der Konstruktion bildet, davon beeinflusst wird, ob es sich bei dem im Possessivsuffix kodierten Referenten um Sprecher, Hörer oder eine dritte Person handelt:

I conclude that the two types of uses can be modelled using the same semantic mechanisms on the assumption that the salience of objects in the discourse representation is affected by whether they are assumed to be owned by the speaker, the listener, or some other possessor. (Simonenko 2014:2)

Das zweite Kapitel (Simonenko 2014: 2f.) stellt für jede der drei behandelten Sprachen ein eigenes Muster der Verwendung des Possessivsuffixes der dritten Person Singular dar sowie den jeweiligen Kontext, in welchem es verwendet wird. Basis der Analyse bildet auch hier eine Adaption der Definitheits-Kriterien von Hawkins 1978, welche die Schlussfolgerung zulassen, dass der Gebrauch des Possessivsuffixes der dritten Person Singular nicht mit dem definiten Artikel des Englischen gleichgesetzt werden kann:

As an heuristic tool, I examine 3SG uses following, loosely, the classification of "definite" contexts of Hawkins (1978). This, as I will show below, by no means implies that 3SG has the same grammatical status as the English definite article. (Simonenko 2014: 2) 
Für das Chantische kommt Simonenko zu der Schlussfolgerung, dass nur vorerwähnte Referenten als möglicher Kopf in der Konstruktion infrage kommen (» The only context allowing for non-possessive uses is the presence of an anaphoric antecedent « Simonenko 2014: 4). Diese Verwendung bezeichnet Simonenko als »anaphoric antecedent «. Andere anhand der Kriterien von Hawkins 1978 erarbeitete Kategorien wie »known group«, "local uniqueness« und "global uniqueness" sind laut Simonenko $2014 \mathrm{im}$ Chantischen nicht belegt (Simonenko 2014: 4). ${ }^{59}$

Das dritte Kapitel (Simonenko 2014: 6f.) befasst sich mit der bisherigen Analyse dieser Verwendungen des Possessivsuffixes der dritten Person Singular in der Forschung (zum Beispiel Collinder 1955; Fraurud 2001; Nikolaeva 2003; Gerland 2011 ${ }^{60}$ ) und weist auf die Diskrepanzen der bisherigen Analysen hin, wenn diese einzelsprachlich betrachtet werden.

Im vierten Kapitel (Simonenko 2014: 7f.) stellt Simonenko 2014 Herangehensweise und Analysemethode vor. Für das Chantische stellt sie dabei fest, dass das Possessivsuffix der dritten Person Singular eine anaphorisch-vorangehende Relation (»anaphoricantecedent relation «, Simonenko 2014: 8) ausdrückt, jedoch nur dann, wenn der Referent, der das Suffix trägt, im Diskurs vorerwähnt ist:

The function outputs the most salient individual with the relevant property among those introduced in the discourse. (Simonenko 2014: 8)

Im fünften Kapitel (Simonenko 2014: 11f.) erfolgt ein Rückbezug auf und die Verknüpfung mit sogenannten prototypischen Funktionen des Possessivsuffixes - den Ausdruck von Possession. Hier folgt Simonenko 2014 nicht dem gängigen Argumentationsverlauf ausgehend vom Ausdruck von Possession und der Kategorisierung jener Verwendungen, die dort nicht subsumiert werden können, als sekundäre Funktion -, sondern sie geht von der Tatsache aus, dass in den drei in ihrem Aufsatz behandelten Sprachen ein Suffix mit demselben ur-uralischen Ursprung (»cognate suffixes«, Simonenko 2014: 11) dieselbe nicht-possessive Kategorie ausdrücken kann und dieses Suffix gleichzeitig in allen drei Sprachen auch für die sogenannten prototypischen Funktionen verwendet wird und somit hier ein Zusammenhang bestehen muss. Diesen Zusammenhang sieht sie in der Relationalität (»functional possessive« Simonenko 2014: 11):

It seems that we can say that the role of the possessive suffix is to pick an individual out of a set of individuals with the relevant property belonging to some person. (Simonenko 2014: 11)

Abgeleitet auf die nicht-possessive Verwendung von Possessivsuffixen entspricht die Relationalität der Salienz des Referenten im Diskurs (verglichen »in Komi the 3SGfunction picks out the most salient individual according to a given contextual salience ranking « Simonenko 2014: 11). Berücksichtigt man dazu noch die Possessivsuffixe der ersten und zweiten Person Singular, ergibt sich laut Simonenko 2014 folgendes hierarchisches Schema: 
Individuals owned by the speaker and by listener occupy the top-most and the second top-most ranges on the salience ranking, while all other individuals have lower ranks. (Simonenko 2014: 11)

Demnach kann für beide Verwendungen - die possessive und nicht-possessive - festgehalten werden, dass der mit dem Possessivsuffix der dritten Person Singular markierte Referent durch seine Verbindung mit einem salienten Referenten (formal markiert durch das Possessivsuffix, semantisch entweder als Besitz oder Assoziation interpretiert) selbst salienter wird (Simonenko 2014: 11).

Abschließend postuliert Simonenko 2014 zwei referenzbezogene Kategorien, in welche die Funktion des Possessivsuffixes der dritten Person Singular je nach Sprache einzuordnen ist: (a) basierend auf Salienz im Komi und Chantischen und (b) basierend auf einer festgelegten Zugehörigkeit (»set-membership based «). Dies kann auch für die possessiven Lesarten angenommen werden:

In other words, I proposed that the salience ranking relevant for the semantics of possessive suffixes is partially derived from the hierarchy of "possessors". Assuming that $[[1 \mathrm{SG}]]$ and $[[2 \mathrm{SG}]]$ are associated with specific ranges on the ranking (individuals owned by the speaker/listener), while [[3SG]] covers the rest, it is unsurprising that it is $3 \mathrm{SG}$ that is used in both possessive and non-possessive contexts. (Simonenko 2014: 13)

Simonenkos Aufsatz enthält vielversprechende, bislang nicht oder nur zum Teil berücksichtigte Aspekte bei der Re-Analyse der Possessivsuffixe: Es werden zwar Gemeinsamkeiten in der Verwendung in den uralischen Sprachen benannt, aber auch auf die Möglichkeit einzelsprachlicher Entwicklungen verwiesen. Des Weiteren erwähnt sie Kontext und Vorerwähntheit als wichtigen Faktor und bezieht in ihrer Analyse Salienz und Diskurs mit ein. Außerdem beschreibt sie zu Beginn des Aufsatzes das Possessivsuffix als referentiellen Marker (Simonenko 2014: 1) und verweist auf unterschiedliche Funktionen je nach im Possessivsuffix kodierter Person.

All diese Aspekte kommen meines Erachtens jedoch im weiteren Argumentationsverlauf nicht ausreichend zum Ausschlag, stattdessen wird auch hier wieder der Definitheits-Ansatz nach Hawkins 1978 zur Grundlage der Analyse - trotz Hinweis auf die vielen weiteren ausschlaggebenden Faktoren. Der Kontext beispielsweise bezieht sich auf die Vorerwähntheit des Kopfes der Konstruktion, nicht auf die im Possessivsuffix kodierte Person (analog zur Analyse eines definiten Artikels), die im Possessivsuffix kodierte Person wird, was die Auswirkungen auf den markierten Referenten betrifft, berücksichtigt, nicht jedoch die kodierte Person selbst (topikale Rollen oder Sprachaktteilnehmer).

\subsubsection{Die Forschungsansätze zum Possessivsuffix in anderen uralischen Sprachen}

Der Forschungsstand zum Possessivsuffix in den ugrischen Sprachen wurde im Verlauf des Kapitels auch immer wieder in Relation zum Forschungsstand in der Uralistik allgemein und in anderen Sprachzweigen gesetzt. Zuletzt soll deshalb noch auf neueste Forschungen zum Possessivsuffix in anderen unmittelbar den ugrischen Sprachen 
benachbarten Sprachzweigen verwiesen werden. Besonders sind hier die Forschungen im permischen Sprachzweig zu erwähnen: Neben Abhandlungen wie Suihkonen 2005, Leinonen 2006, und Edygarova 2009, auf welche nur am Rande verwiesen wird, sind dies Edygarova 2013 und Klumpp 2013. Bei letzteren handelt es sich um Vorträge der Konferenz »Grammar and Context IV: New Approaches to the Uralic Languages", welche 2013 in Tartu stattgefunden hat und Ausgangspunkt einer zukünftigen sprachzweig-übergreifenden Forschung zum Possessivsuffix darstellt. Den ersten Schritt bildet ein Sonderband zur Konferenz mit dem Themenschwerpunkt der referentiellen Verweismittel in den uralischen Sprachen, welche auch ein Aufsatz der Verfasserin der vorliegenden Arbeit zum Possessivsuffix im Nord-Mansischen enthält (Janda 2015). Da die sprachzweig-übergreifende Forschung damit noch am Anfang steht, wird in der vorliegenden Arbeit auf den Erkenntnisgewinn hingewiesen; sie kann jedoch zum derzeitigen Zeitpunkt nicht als Grundlage für die vorliegende Arbeit herangezogen werden.

Zuletzt sei noch auf den anderen unmittelbar benachbarten Sprachzweig, nämlich auf die samojedischen Sprachen hingewiesen, dort seien die Publikationen von Körtvély 2009 und Siegl 2015 erwähnt.

Wie anhand des Forschungsstandes skizziert ist die Beschreibung der Possessivsuffixe und seiner Verwendungen bislang primär onomasiologisch erfolgt. Die vorliegende Arbeit hingegen ist semasiologisch ausgerichtet, das heißt von der Form ausgehend, deren Funktion je nach Umgebung, in welcher sie verwendet wird, beschrieben wird. Im Gegensatz zum Großteil der bisherigen Abhandlungen erfolgt dabei keine Zweiteilung in possessive und nicht-possessive/definite Funktionen. Es wird auch nicht von einer Grammatikalisierung des Possessivsuffixes zu einem Definitheitsmarker ausgegangen und folgt in dieser Hinsicht dementsprechend den Ansätzen von Fraurud 2001 oder Gerland 2014a. Dabei wird auch in einem gewissen Umfang die Ko-Okkurrenz von Demonstrativpronomina (sowie dem definiten Artikel im Ungarischen), eines der Hauptargumente gegen eine Grammatikalisierung zum Definitheitsmarker, in der Analyse mitberücksichtigt.

Es wird weiterhin versucht, die allen Possessivkonstruktionen zugrunde liegenden Faktoren herauszuarbeiten, indem der im Possessivsuffix kodierte Referent und dessen Realisierung in der Konstruktion im Fokus der Analyse steht und damit auch die Textumgebung mit einbezogen wird. Zweitens spielt dementsprechend die kodierte Person und die Verknüpfung des Kopfes der Konstruktion mit einem Sprechaktteilnehmer oder einem (topikalen) Referenten im Text eine grundlegende Rolle bei der Analyse. Da nicht nur das Possessivsuffix der dritten Person Singular, sondern das gesamte Paradigma Gegenstand der Analyse ist, unterscheidet sich die vorliegende Arbeit auch in diesem Aspekt von den bisherigen Werken, in welchen die Possessivsuffixe der zweiten Person Singular und das der ersten Person Singular kaum mehr Betrachtung erfahren haben als ein kurzer, vergleichender Verweis (die einzige nennenswerte Ausnahme bildet hier Schlachter 1960).

Die Kategorisierung der in der Konstruktion ausgedrückten Relationen, und insbesondere die Zuweisung eines tatsächlichen Besitzers, oder auch die (Un)Trennbarkeit der Relation bzw. die Konzeptualisierung von Worttypen wie bei Gerland 2014a oder Barker 1995 spielen eine untergeordnete Rolle. 
Stattdessen werden Aspekte wie Referenz und Referentialisierung sowie die Informations-Strukturierung im Text zur Analyse herangezogen. Damit greift die vorliegende Arbeit den aktuellsten Forschungsrahmen von Kognition und Pragmatik zur Possession auf und stellt dies im Rahmen eines text-linguistischen bzw. referenz-linguistischen Ansatzes dar. Der zugrunde liegende theoretische Rahmen wird im folgenden Kapitel beschrieben. 


\section{Theoretische Grundlagen}

Die vorliegende Arbeit ist weitestgehend als framework-free (verglichen Haspelmath 2008) anzusehen, das heißt, die Possessivkonstruktionen sollen aus sich heraus beschrieben werden und nicht in sogenannte prototypische und nicht-prototypische Verwendungen oder Ähnliches kategorisiert werden. Auch die Gleichsetzung von Possessivsuffix und Possessivpronomina, wie sie in manchen typologischen Übersichten erfolgt, oder gar der Vergleich von Possessivsuffixen und anderen grammatischen Markern in Possessivkonstruktionen haben dazu geführt, dass wichtige inhärente Eigenschaften des Possessivsuffixes in der bisherigen Forschung nicht oder nicht in der richtigen Gewichtung betrachtet worden sind (siehe hierzu Kapitel 2). Ein Vergleich mit den Strukturen, welche ähnliche Funktionsweisen in anderen Sprachen ausdrücken, kann durchaus hilfreich und informativ sein, sollte jedoch erst nach der sprachinternen Beschreibung und nur für Teilaspekte erfolgen und nicht von vornherein, um keine bestimmte Erwartungshaltung zu wecken. Damit versucht die vorliegende Arbeit, den Einfluss linguistischer Theorien auf die Analyse in einem geringen Umfang zu halten und Haspelmaths Rat zu folgen, die Theorie auf ein kleines Set von immanenten grammatischen Kategorien und Relationen (»substantive universals«) und eine einfache grammatische Architektur (»formal universals») zu beschränken (verglichen Haspelmath 2008: 344f.).

Es ist demnach hauptsächlich der Bereich der Terminologie, in welchem die vorliegende Arbeit an gewisse linguistische Theorien angelehnt ist. Des Weiteren ist es unumgänglich, sich sowohl mit den zugrunde liegenden Ansätzen der neueren Forschungen zu befassen als den zugehörigen theoretischen Hintergrund der gewählten Analysemethoden zu berücksichtigen. Die zwei vorherrschenden linguistischen Theorien sind dabei zum einen die Kognitive Linguistik, und hier insbesondere der Ansatz der Referenzpunkt-Konstruktion nach Langacker 1993 und 1995, und die sogenannte Functional (Discourse) Grammar bzw. darauf basierende Forschungslinien. Functional Grammar und Kognitive Linguistik sind nicht nur nicht gegensätzlich zueinander, sondern ergänzen sich gegenseitig und werden daher in manchen Fällen sogar als gemeinsame Forschungsgrundlage betrachtet (Elsen 2014: 205).

Theorie wird demnach in der vorliegenden Arbeit nach Haspelmath 2008 als abstraktes Modell bzw. Metasprache zur Sprachbeschreibung (»descriptive framework«) verstanden, und die Sprachbeschreibung (»description«) bzw. Analyse als »Charakterisierung grammatischer Regelmäßigkeiten einer bestimmten Sprache» (»characterization of grammatical regularities of particular languages« Haspelmath 2008: 344).

\subsection{Kognitive Linguistik}

Die Kognitive Linguistik ist eine "Ende der 50er Jahre in den USA entstandene interdisziplinäre Forschungsrichtung, die sich mit der Untersuchung mentaler Prozesse bei Erwerb und Verwendung von Wissen und Sprache beschäftigt« (Bußmann 2002: 350), wobei Kognitive Linguistik eher ein Sammelbegriff für verschiedene Sprach- und 
Kognitionstheorien - darunter die Konstruktions-Grammatiken von Croft 2001 oder Goldberg 1995 und die Kognitive Grammatik von Langacker (Langacker 2008a) - denn eine einheitliche Theorie ist (Ziem 2008: 1). »Als Begründer der Kognitiven Linguistik gelten Charles J. Fillmore (Frame-Semantik), George Lakoff (Prototypen, Basisausdrücke Metaphern/Metonymie), Ronald W. Langacker (Kognitive Grammatik) und Leonard Talmy (Kognitive Semantik)«(Elsen 2014: 206). Die Hauptprinzipien, auf welchen die Kognitive Linguistik basiert, sind Kategorisierung bzw. Schematisierung, Konzeptualisierung und die Gebrauchsbasiertheit (verglichen Ziem 2008: 2). Das unbewusste und automatische Zusammenfassen (Kategorisierung) von Vorgängen, Bewegungen oder Relationen zwischen Entitäten erleichtert die sprachliche Verarbeitung (verglichen Elsen 2014: 207). Die Grammatik (Konzeptualisierung) wird wie Lexeme auch als Form-Bedeutungs-Paarung verstanden, die Syntax ist damit "nicht autonom, sondern basiert auf Bedeutung (das umfasst auch pragmatische Aspekte) und damit auf kognitiven Modellen« (Elsen 2014: 209). Erst durch den Gebrauch der Sprache entstehen Bedeutung und grammatische Strukturen, welche sich auch wieder verändern können (Ziem 2008: 2). Das Prinzip der Kognitiven Linguistik kann man wie folgt auf das Grundlegende zusammenfassen: Informationen werden wahrgenommen, verarbeitet, gespeichert und dann versprachlicht, die Sprache ist daher Mittel zur »Organisation, Verarbeitung und Übermittlung von Wissen «(Elsen 2014: 206). Dabei entstehen durch die Kategorisierung lose Anordnungen, welche während des Denkens und Sprechens vom Sprecher und Hörer zum unmittelbaren Verstehen der Proposition konstruiert und modelliert werden, sogenannte Konzepte:

Ein Konzept ist eine Konstellation, eine kognitive Einheit von Wissen oder eine mentale Beschreibung, die wir aus unseren wahrgenommenen Eindrücken destillieren. (Kern 2010: 16)

In Modellen wie sogenannten frames wird dargestellt, »inwiefern Konzepte zueinander in Verbindung treten können [...] um mögliche Strukturierung von Wissen zu veranschaulichen « (Kern 2010: 16). Abgeleitet auf die Syntax zum Beispiel ist ein verbales Prädikat zusammengesetzt aus dem Prozess der Handlung und den Rollen der Mitspieler, das heißt von Subjekt und Objekt(en). Ein frame bildet damit stabile Ereignisund Handlungsschemata. In den Frames können alle Arten von semantischen Rollen repräsentiert werden, welche in den jeweiligen syntaktischen Rollen realisiert werden (Barsalou 1992: 29). Jedoch gibt nicht nur das Verb und seine Valenz »die offenen Stellen (slots) in solch einem Rahmen vor, sondern auch die Gesprächssituation und die Kultur « (Elsen 2014: 206). Frames und deren Bestandteile stehen miteinander in Relation und »können sich gegenseitig aktivieren« (Elsen 2014: 207). Ähnliche Begriffe sind »mental spaces«, »concepts«, »complex categories « oder »domains ${ }^{61}{ }^{61}$ Allen gemeinsam ist die Annahme, dass Bedeutung im konkreten wie übertragenen Sinne im Körper verankert ist und wir dadurch mit conceptual archetypes (»konzeptuellen Archetypen«) und image schemas (»Bild-Schemata») Sachverhalte ausdrücken und verstehen

61 Je nach Richtung innerhalb der Kognitiven Linguistik bezeichnen diese Begriffe ähnliche Phänomene, sind jedoch nicht synonym zueinander. Die vorliegende Arbeit strebt allerdings keine detailliertere Diskussion der einzelnen Forschungsrichtungen an, sondern wird sich auf die Begrifflichkeiten nach Langacker (siehe Kapitel 3.1) festlegen. 
können. So verwenden wir beispielsweise für Richtungs- und Ortsangaben das Schema Source - Path - Goal oder den Körper als Konzept (,oben $\sim$,Kopf'; , unten ' ,Fuß'). Auch metonymische Beziehungen wie Teil-Ganzes-Relationen sind konzeptuelle Archetypen (Langacker 1993: 3), mit anderen Worten:

Cognitive linguistics is an exploration of the fabric of meaning, woven thread by thread from bodily experience and embroidered by metaphor. (Janda 2006: 11)

Die Relation, welche zwischen einem image schema und einem conceptual archetype erstellt werden kann, wird in der Kognitiven Grammatik als ReferenzpunktKonstruktion bezeichnet. Die Kognitive Grammatik wurde von Ronald W. Langacker ab den 1970er-Jahren erarbeitet und ist mittlerweile das "am weitesten entwickelte Modell der Kognitiven Linguistik « (Elsen 2014: 212). Die Kognitive Grammatik geht von Domänen aus, wobei es sich nicht um Konzepte an sich handelt, sondern um Erfahrungsbereiche, aus welchen heraus die Konzeptionierung entsteht (»these [cognitive domains, G.J.] are not themselves concepts but irreducible realms of experience within which conception can emerge « Langacker 2008b: 98), und werden unterteilt in "basic" (Raum, Zeit, etc.) und "non-basic concepts«. Unter letztere fallen auch solche Konzepte, welche durch Kontext und Diskurs erfasst werden (Langacker 2008b 98). Dadurch ist der in einem Konzept ausgedrückte Inhalt immer komplexer als die Summe der durch die Lexeme beinhalteten Domänen:

The main point is simply that an expression's conceptual content is complex, multifaceted, and not limited to the domains evoked by its component lexical items. (Langacker 2008b: 98)

Die Referenzpunkt-Konstruktion beschreibt die gleichzeitige Aktivierung eines bestimmten conceptual archetype durch ein bestimmtes image schema (Langacker 1993: 4). Referenzpunkt-Konstruktionen bestehen daher aus einem Konzeptualisierer (»conceptualizer«), welcher eine Relation zwischen einem Target (»target entity«) und einem Referenzpunkt (»reference point «) erstellt. Als Konzeptualisierer dient meistens der Sprecher, Target und Referenzpunkt sind Bestandteil einer bestimmten dominion (der Umfang an Konzepten, welche als Referenzpunkt und Target fungieren können).

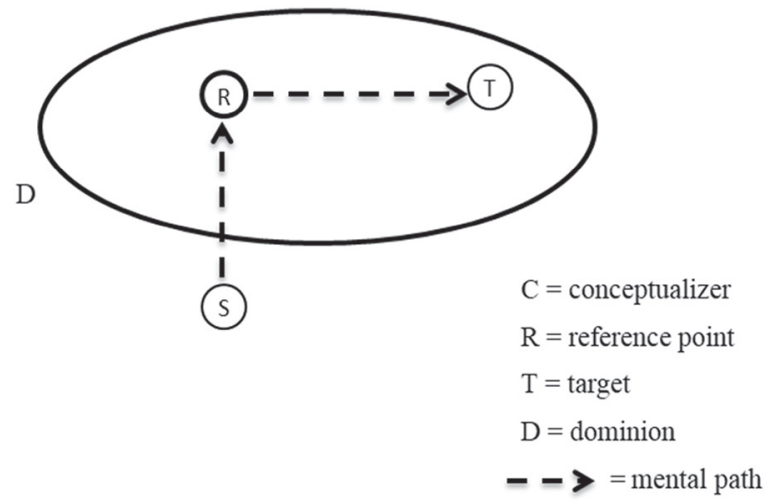

Abbildung 5: Das Referenzpunkt-Modell nach Langacker 1993: 6. 
Referenzpunkte sind in ihrer ersten Erwähnung bei Rosch 1975 als Idealtypen definiert, welche als Ankerpunkt in der Auffassung dienen (»ideal types that serve as anchoring points in perception « Tribushinina 2008: 25) und als Stimuli fungieren, um andere Entitäten damit in Relation zu setzen. Diese Eigenschaft kann der Entität, welche als Referenzpunkt fungiert, inhärent sein oder zum Beispiel durch den Kontext erfolgt sein (Langacker 1993: 6). Damit sind sie asymmetrisch, das heißt, ein Referenzpunkt kann ein Target verankern, aber nicht zwangsläufig im Umkehrschluss ein Target einen Referenzpunkt. Mit anderen Worten, die Referenzpunkt-Konstruktion illustriert die Idee, dass wir bestimmte Konzeptionen heranziehen, um mental Kontakt zu anderen Entitäten herzustellen:

The reference-point model is simply the idea that we commonly invoke the conception of one entity for purposes of establishing mental contact with another. (Langacker 1995: 58)

Wird mentaler Kontakt hergestellt (»establishing mental contact«), wird eine Entität aufgegriffen und dadurch zu einem bestimmten individuellen Referenten (»to single it out for individual conscious awareness " Langacker 1995: 58). Dies geschieht meist unbewusst (Langacker 1993: 5). Possession wird in der Kognitiven Linguistik als eine universale Kategorie definiert, welcher das Referenzpunkt-Modell inhärent ist (Langacker 1995: 61); Besitzverhältnisse, Teil-Ganzes-Relationen oder Verwandtschaftsbeziehungen werden als Prototypen dieser Kategorie angesehen:

The internal identification mechanism of possessive NPs and its 'anchoring' of referents has been at the core of the analysis of possessives as reference-point constructions, developed within the framework of Cognitive Linguistics by Langacker. (Willemse 2009: 17)

Als Referenzpunkt fungiert dabei der Possessor, das Possessum als Target, als dominion fungieren die in der Dimension Possession subsumierten Relationen, aber auch beispielsweise der Diskurs respektive Kontext. In der vorliegenden Arbeit wird das Referenzpunkt-Modell als Beschreibungsgrundlage für die Possessivkonstruktion übernommen, im Folgenden werden daher die Begriffe »Referenzpunkt« und »Target« für »Possessor und $»$ Possessum $«$ verwendet. ${ }^{62}$

\subsection{Funktionale Grammatik}

Das Modell der Funktionalen Grammatik basiert ebenfalls auf dem Gebrauch von Sprache. Ähnlich wie bei der Kognitiven Linguistik werden unter dem Begriff der Funktionalen Grammatik verschiedene Konzepte zusammengefasst, deren gemeinsame Grundlinie die Annahme ist, dass sich die Form aus der Funktion ableitet, das heißt, je nach der Bedingung, unter welcher eine Äußerung entsteht, ergibt sich deren Struktur

62 Die Terminologie beschränkt sich dabei auf Konstruktionen, in welchen zwei Referenten miteinander in Bezug gesetzt werden. Wird nur ein Referent in der Konstruktion kodiert, wird weiterhin von Modifizierer und Kopf gesprochen. 
(Elsen 2014: 189). Der gesamte in der Funktionalen Grammatik beschriebene Zusammenhang von semantischen, syntaktischen und pragmatischen Rollen, Textgefüge und Äußerungssituation inklusive Kommunikationsabsichten der Sprechaktteilnehmer beschreibt damit sämtliche für die text-linguistische Methode der vorliegenden Arbeit relevanten Aspekte, welche im Folgenden genauer thematisiert werden.

Grundlegende Werke innerhalb der Functional Grammar sind beispielsweise das gleichnamige zweibändige Werk von Dik (Dik 1997a und Dik 1997b), die »Functional Discourse Grammar« nach Hengeveld/Mackenzie 2008 und die »Role and Reference Grammar « nach Van Valin 1993. Mit Funktion sind dabei einerseits die Satzgliedfunktionen wie Subjekt oder direktes Objekt gemeint, aber auch die »Aufgaben, die Sprachen innerhalb der Kommunikation zufallen « (Elsen 2014: 189). Die Funktionale Grammatik ist daher pragmatisch ausgerichtet, zentrale Aspekte bilden die Sprechaktteilnehmer und die Sprechsituation inklusive sprachlicher wie auch nicht-sprachlicher Faktoren (Elsen 2014: 190).

Ihren Ursprung hat die Funktionale Grammatik in der Prager Schule ${ }^{63}$ und führt damit die Theorie der sogenannten Funktionalen Satzperspektive weiter (Elsen 2014: 190). Die Funktionale Satzperspektive bezeichnet die »Gliederung des Satzes unter dem Aspekt seiner Mitteilungsfunktion« (Bußmann 2002: 231) und bildet damit einen »der zentralen Arbeitsbereiche der Funktionalen Grammatik« (Elsen 2014: 190). Die bekannteste Einteilung der Mitteilungsfunktion ist die Thema-Rhema-Gliederung (verglichen Elsen 2014: 190). Das Thema ist der Teil eines Satzes, welcher bekannt ist »und damit einen relativ geringen Mitteilungswert hat [...]. Das Rhema dagegen transportiert einen relativ hohen Mitteilungswert« (Kern 2010: 70), indem es neue Informationen enthält.

Forschungslinien innerhalb oder aufbauend auf der Funktionalen Grammatik, welche ihren Ursprung im amerikanischen Raum haben - wie zum Beispiel Li (Hg.) 1976 oder Givón 1983 - bevorzugen hingegen die in der Pragmatik etablierten Begriffe »Topik« und »Fokus«. Die Pragmatik ist eine relativ junge linguistische Teildisziplin, welche sich mit Äußerungssituationen befasst und deren Informations-Struktur analysiert, das heißt mit der Gliederung der Satzaussage nach kommunikativen Gesichtspunkten (verglichen Bußmann 2002: 534). Das Topik des Satzes ist das, worüber im Satz etwas ausgedrückt wird, das heißt, worüber der Sprecher eine Aussage tätigt. Dabei kann, muss das Topik des Satzes aber nicht automatisch die bekannte gegebene Information repräsentieren, dies ist zum Beispiel insbesondere zu Beginn einer Erzählung der Fall. Als Fokus wird im Allgemeinen eine Hervorhebung bzw. Betonung eines bestimmten Teils der Äußerung bezeichnet. In den meisten Fällen ist die Information im Fokus des Satzes neue Information, er darf jedoch nicht per se gleichgesetzt werden mit dem Satzteil, welcher die neue Information enthält:

There are many cases in which a constituent that refers to something previously mentioned is in focus. (Krifka 2008: 256)

Somit ist es wichtig, zu verdeutlichen, dass die neue Information nicht als Fokus des Satzes fungiert, sondern im Fokus des Satzes stehen kann. Die Forschung zum Fokus

63 Die Prager Schule ist eine Richtung des europäischen Strukturalismus und geht auf den 1926 gegründeten Cercle Linguistique de Prague zurück (Bußmann 2002: 533). 
nimmt daher immer ein Set von Alternativen an, aus welchen das fokussierte Element das für die Interpretation relevante ist (verglichen Krifka 2008: 247). Dabei werden verschiedene Arten von Fokus und unterschiedliche Voraussetzungen je nach Art des Fokus angenommen. Krifka unterscheidet des Weiteren zwei Arten von Fokus, den pragmatisch-gebrauchten, welcher für das common ground management eingesetzt werden kann - das heißt für die »kommunikativen Ziele der Sprechakteilnehmer« (»communicative goals of the participants« Krifka 2008: 249) - und den semantischgebrauchten Fokus, welcher sich auf den tatsächlichen Inhalt der Aussage bezieht (Krifka 2008: 249). Die detaillierten Forschungen zum Fokus und Fokusgebrauch beinhalten auch immer eine Diskussion zum Wahrheitsgehalt einer Aussage. Für den theoretischen Rahmen der vorliegenden Arbeit genügt es jedoch - auch aufgrund der verwendeten Korpus-Materialien (keine Tonaufnahmen, ein relativ einheitliches Textgenre, siehe dazu Kapitel 4.1) - von einer ganz vereinfachten Definition des Fokus auszugehen, welche nicht den Anspruch erhebt, den Aspekt des Fokus vollständig beschreiben zu können. Fokussierung wird definiert als die Intention des Sprechers, einen bestimmten Satzteil hervorzuheben. Die Funktionen des Fokus sind demnach Antworten auf Fragen (»the wh-part of a constituent question« Krifka 2008: 250), Abgrenzen des Inhalts der Satzaussage auf einen bestimmten Referenten, Korrigieren von Satzaussagen, Bestätigen von Satzaussagen sowie das Hinweisen auf parallele Strukturen in Satzabfolgen (verglichen Krifka 2008: 252f.). Die naheliegende Möglichkeit, Fokus auszudrücken, liegt auf der phonologischen Ebene: durch Intonation. Aber auch auf der morpho-syntaktischen Ebene gibt es Mechanismen, um Fokussierung im geschriebenen Text anzuzeigen, beispielsweise kann es eine bestimmte Position im Satz geben, dessen Bestandteil immer im Fokus steht, oder der Satzteil kann mit einer Fokuspartikel versehen werden. In den ugrischen Sprachen geht man von einer präverbalen Fokusposition aus. Die Fokussierung wird in Kapitel 5.2 detaillierter im Hinblick auf die Possessivsuffixe beschrieben.

Die Verwendung der Begriffspaare von Thema-Rhema bzw. Topik-Fokus ist größtenteils den unterschiedlichen Traditionen in Europa und Amerika geschuldet, dennoch dürfen beide Paare nicht als Übersetzung und damit als synonym angesehen werden. Leider sind sämtliche Termini bis heute nicht eindeutig definiert und voneinander abgegrenzt (verglichen Elsen 2014: 194). Viele Forschungsansätze setzen alle drei Begrifflichkeiten »Thema-Rhema «, »Topik« und »Fokus« in Relation zum OrganonModell nach Bühler [1934] 1982 und unterscheiden drei Ebenen je nach Perspektive.

Das Organon-Modell von Bühler geht von der Ausdrucks-, Appell- und Darstellungsfunktion aus (Heinemann 2008: 119). Die Thema-Rhema-Gliederung kann dabei als satzexterne Ebene angesehen werden, welche sich auf die Satzaussage selbst bezieht und das zentrale Thema und die Aussagen darüber (Rhema) einordnet (verglichen Elsen 2014: 194), und besitzt damit Darstellungsfunktion.

Abgeleitet auf die dem Satz übergeordnete Einheit, den Text, entspricht das Thema der »Grundinformation «, »der Entfaltung eines Inhaltskerns« nach Brinker (Brinker 2005: 22). Satzintern bildet das Topik zusammen mit dem Comment (Kommentar) die faktische Ebene mit Ausdrucksfunktion und der Fokus zusammen mit dem Background (Hintergrund) die sprecherbezogene Ebene mit Appellfunktion. Die faktische Ebene bezieht sich dabei auf den oder die konkreten Referenten und die Aussagen 
darüber. Aus diesem Grund wird in der vorliegenden Arbeit in Anlehnung an Dik 1978 der Begriff »Topik « im Folgenden als pragmatische Rolle Topik verstanden, welche sich auf einen konkreten Referenten bezieht. Diese Rolle kann in verschiedenen Arten realisiert werden, zum Beispiel als Diskurs-Topik, Paragraphen-Topik oder Satz-Topik, welche eine topikale Hierarchie bilden. Das Diskurs-Topik ist der Referent, welchem der Sprecher seine Hauptaufmerksamkeit widmet, und gewöhnlich ist ein Diskurs-Topik in die gesamte Handlung der Erzählung involviert. ParagraphenTopiks existieren nur durch ihre Beziehung zum Diskurs-Topik, das heißt, sie treten nur in Situationen zusammen mit dem Diskurs-Topik auf - zum Beispiel der Antagonist des Haupthelden. Sie treten nicht in der gesamten Erzählung auf, aber in einem oder zwei Paragraphen (verglichen Givón 1983: 22f.). Da das Diskurs-Topik im Hauptaugenmerk des Sprechers ist, gibt es nur einen Diskurs-Topik pro Erzählung, mehrere Paragraphen-Topiks sind dagegen jedoch in einer Erzählung möglich, wobei die Anzahl insofern begrenzt wird, als wir nur eine eingeschränkte Anzahl in »aktivem Zustand erhalten können« (Kern 2010: 72). Manchmal wechselt auch die Aufmerksamkeit des Sprechers zwischen topikalen Rollen, und ein bisheriger Paragraphen-Topik wird zum Diskurs-Topik und umgekehrt. Satz-Topiks sind die am wenigsten wichtigen Topiks in der Hierarchie und kommen nur in wenigen Sätzen in einer Erzählung vor. Dem Topik gegenüber steht das Comment, das Informationszentrum des Satzes: Der Teil des Satzes, welcher das Wissen über das Topik erweitert, das Topik dementsprechend kommentiert. Diese Ebene wird, insbesondere mit Zuhilfenahme der topikalen Rollen zur Strukturierung des Textes eingesetzt (siehe dazu auch Abschnitt Progression).

Fokus und das Gegenstück, der Background (der Teil des Satzes, welcher nicht hervorgehoben wird) bilden wiederum eine Ebene, welche in Zusammenhang mit dem Sprecher steht, und beinhaltet das, was der Sprecher hervorheben will. Zusammen mit der Topik-Kommentar-Ebene erfolgt hier die Informations-Strukturierung. In der vorliegenden Arbeit stehen daher diese beiden Komponenten im Mittelpunkt.

Sätze entstehen durch das Zusammenwirken von Morphologie, Syntax und Phonologie, das heißt, den Referenten werden syntaktische Rollen zugewiesen, welche mittels morphologischer Marker und/oder Satzstellung markiert werden; wird die Satzaussage einem Hörer mitgeteilt, wird sie zudem phonologisch realisiert. Zusätzlich muss dieses sprachliche Gebilde aber in einen Diskurszusammenhang gebracht werden, um einen kommunikativen Wert zu haben. Der Erfolg der Kommunikation ist darin begründet, dass der Sprecher den Wissensstand des Empfängers richtig einschätzt und daran anknüpft. Dieses Zusammenwirken geschieht auf der linguistischen Analyseebene der Pragmatik, wobei Informations-Struktur das Zuschneiden einer Aussage durch den Sprecher, um den jeweiligen Bedürfnissen des Empfängers gerecht zu werden, bezeichnet. Dabei muss der Sprecher das richtige Verhältnis zwischen gegebener und neuer Information herstellen. Besteht eine Satzaussage nur aus neuer Information, kann der Hörer gegebenenfalls nicht folgen. Beinhaltet die Satzaussage bekannte Information, so kann die neue Information darauf aufgebaut werden. Ist jedoch zu viel bekannte Information enthalten, so ist der Mitteilungswert der Aussage ebenfalls fraglich (verglichen Kern 2010: 70). Die Informations-Struktur ist somit der ausschlaggebende Faktor für den Mitteilungswert des Satzes: 
[Information structure is, G.J.] that component of sentence grammar in which propositions as conceptual representations of states of affaires are paired with lexico-grammatical structures in accordance with the mental states of interlocutors who use and interpret these structures as units of information in given discourse context. (Lambrecht 1994a: 5)

Grundsätzlich bei allen Ansätzen ist somit die Unterscheidung von Gegebenem und Neuem, und dass Sätze nicht isoliert betrachtet werden können, sondern als Teil eines »Text- oder Kommunikationszusammenhangs « (Elsen 2014: 196). Weiterhin wird angenommen, dass dem Text eine Organisation zugrunde liegt, welcher für das Textverständnis entscheidend ist. Diese Verbindung wurde zuerst bei Daneš 1970 als »thematische Progression« bezeichnet und bildet heute eine wichtige Forschungsgrundlage der Textlinguistik.

\subsubsection{Textlinguistik}

Die sogenannte Textlinguistik befindet sich als relativ junge Disziplin an der Schnittstelle zwischen Linguistik, Literaturwissenschaft und Rhetorik, es handelt sich sozusagen um eine Textwissenschaft, welche sich in den letzten rund 25 Jahren als eigenständiger Forschungszweig etabliert hat, aber auch auf die Funktionale Grammatik zurückgeht. Sie befasst sich mit Strukturen und Prozessen der sprachlichen Einheit Text (Vater 2001: 8). Ein Text ist eine formal begrenzte, schriftliche Äußerung, die mehr als einen Satz umfasst; es handelt sich demnach um eine miteinander verbundene Kette von Sätzen (Fix 2008: 21). Der Begriff »Text «schließt aber auch mündliche Kommunikationsvorgänge mit ein und ist als Kommunikationsbestandteil dem Sprechakt benachbart (Vater 2001: 14). Der Begriff »Diskurs« ist ein aus dem Englischen übernommener Oberbegriff für verschiedene Aspekte von Text (Grenoble 2006: 1). Gegenstand der Analyse der vorliegenden Arbeit sind schriftliche Formen von Text, von denen jedoch der Großteil schriftlich fixierte Belege mündlicher Erzähltradition ausmacht. In der vorliegenden Arbeit wird Text daher sowohl mündlich als auch schriftlich verstanden und synonym zum Begriff Diskurs verwendet, wobei der Begriff Text dem vorhandenen Korpusmaterial gerechter wird.

Die sogenannte Diskurslinguistik ist allerdings vor allem im literaturwissenschaftlichen Bereich verankert. Im linguistischen Kontext spricht man eher von der Diskursanalyse, der Erforschung des Gebrauchs von Sprache. Sie basiert auf der Annahme, dass Sprache ohne Kontext - sprachlich und außersprachlich - nicht zu verstehen ist (Grenoble 2006: 1). Diskursanalyse wird in der vorliegenden Arbeit synonym zur Textanalyse verstanden, wobei der Einbezug von außersprachlichen Elementen durchaus in gewissem Umfang Berücksichtigung erfährt, sofern es in Bezug auf das Korpusmaterial angezeigt ist (siehe dazu auch Abschnitt Text-Deixis).

Kriterien der Textualität, das heißt, was die kommunikativen Eigenschaften einer Kette von Sätzen ausmacht, sind unter anderem Kohäsion, Kohärenz, Intentionalität, Situationalität, Akzeptabilität, Informativität, Intertextualität. Weitere Aspekte der Textanalyse befassen sich mit dem Textthema, der Referenz im Text oder dem Textverstehen (Fix 2008: 18). Die vorliegende Arbeit konzentriert sich dabei auf die linguistischorientierten Aspekte der Textlinguistik, das heißt satzübergreifende grammatische, semantische und pragmatische Eigenschaften und entsprechende kognitive Aspekte 
der Textherstellung (Vater 2001: 8). Berücksichtigung finden daher insbesondere Kohäsion und Referenz im Text. Die Behandlung von Textsorten oder eine literaturwissenschaftliche Textanalyse sind hier nicht berücksichtigt.

Eingangs wurden die Mitteilungsfunktion einer Aussage, dargestellt mithilfe der Thema-Rhema-Gliederung, sowie die Organisation im Text, welche ausschlaggebend für das Textverständnis ist, erwähnt. Für beides ist die richtige Einschätzung des Wissenstandes des Hörers durch den Sprecher Voraussetzung, um die Satzaussage entsprechend zu strukturieren. Die Thema-Rhema-Gliederung bestimmt nicht nur den Mitteilungswert eines Satzes, sondern ist auch für die Textherstellung maßgeblich. Der Text besteht aus einer Abfolge von Sätzen, welche nicht nur einzeln einen Mitteilungswert besitzen müssen, sondern zudem einen Zusammenhang, eine Verkettung aufweisen müssen. Im Idealfall bauen die Informationen, welche von Satz zu Satz vermittelt werden, aufeinander auf, wodurch eine »thematische Orientierung « im Text entsteht (Brinker 2005: 46f.). Man spricht dann von Kontinuität, dem Zusammenwirken von Kohärenz und Kohäsion. Die Kohärenz beschreibt dabei »ein mentales Konzept, das aus Sicht des Hörers maßgeblich vom Text selbst, Kontext und den daraus abgeleiteten Schlussfolgerungen abhängt (Hansen 1998: 176). Kohäsion ist die grammatische Verknüpfung von Komponenten des Textes (mithilfe textgrammatischer Regularitäten) (Fix 2008: 21). Damit hat »Kontinuität eine konzeptuelle und eine formale, sprachliche Dimension. Erstere betrifft vor allem informations-strukturelle Aspekte, letztere die Anapher « (Kern 2010: 69). Vom kognitiven Gesichtspunkt aus wird Textverstehen als dynamischer Prozess angesehen, in dessen Verlauf ein kognitives Modell der vermittelten Informationen erstellt wird (»text and discourse processing are dynamic processes during which the reader or listener constructs a cognitive representation of the information in the text or discourse "Sanders/Gernsbacher 2004: 79). Mit der Textanalyse kann die Thema-Rhema-Gliederung eines Textes dargestellt werden. Eines der bekanntesten Modelle ist das eingangs erwähnte der Thematischen Progression von Daneš 1970, welcher die Textstruktur als eine »Sequenz von Themen« bezeichnete:

Die eigentliche thematische Struktur des Textes besteht [...] in der Verkettung und Konnexität der Themen, in ihren Wechselbeziehungen und ihrer Hierarchie, in den Beziehungen zu den Textabschnitten und zum Textganzen sowie zur Situation. (Gansel/Jürgens 2002: 37)

Es gibt fünf Grundtypen der Thematischen Progression: mit linearem Thema, durchlaufendem Thema, abgeleitetem Thema, gespaltenem Thema und mit thematischem Sprung. Bei der linearen Progression wird das Rhema eines Satzes zum Thema des Folgesatzes, das Rhema dieses Satzes wiederum zum Thema des nächsten Satzes usw. Bei der Progression mit einem durchlaufenden Thema wird diesem jeweils ein Rhema zugeordnet, das Thema selbst bleibt über mehrere Sätze konstant.

Die Erzähltradition des Ob-Ugrischen entspricht größtenteils der Progression mit durchlaufendem Thema. Bei der Progression mit abgeleitetem Thema sind die Themen der einzelnen Sätze unterschiedlich, können aber allesamt einem gemeinsamen Hauptthema zugeordnet werden (verglichen Gansel/Jürgens 2002: 38). Andere Theorien, welche sich mit Progression befassen, zum Beispiel nach Jakobson/Halle 1956, gehen von zwei zugrunde liegenden Mechanismen aus: Similarität (Ähnlichkeitsbeziehungen) 
und Kontiguität (Nachbarschaftsbeziehungen) (Kern 2010: 11). Das Prinzip der Kontiguität liegt auch den Possessivkonstruktionen zugrunde.

Abgeleitet auf die Forschungslinien nach Li (Hg.) 1976 und Givón 1983 geht es bei diesen Mechanismen um die sogenannte Topik-Kontinuität bzw. um die Einteilung von Sprachen nach Subjekt-Prädikat-Unterscheidung und Topik-Kommentar-Relation (Elsen 2014: 193). Letztere strukturieren Sätze, indem das Topik kontinuierlich weitergeführt wird, das heißt nach dem Muster der Progression mit einem durchlaufenden Thema. Verkettet werden die einzelnen Sätze durch Weiterführung des Topiks (Similarität) oder mithilfe von Bezugnahme auf das Topik (Kontiguität). Der Topik-Kontinuität zugrunde liegt der Drei-Ebenen-Ansatz, ebenfalls ursprünglich von Daneš eingeführt. Auch die Functional Grammar von Dik (Dik 1997a und Dik 1997b) arbeitet nach den drei Analyse-Ebenen des Satzes (semantisch - syntaktisch - pragmatisch), auf welchen ein Referent gleichzeitig jeweils eine Rolle ausübt (Elsen 2014: 195). Die semantischen Rollen sind beispielsweise Agens, Patiens oder Rezipient. Die syntaktischen Rollen sind Subjekt, direktes oder indirektes Objekt. Auf der pragmatischen Ebene sind es die topikalen Rollen sowie der Kommentar. Die semantischen, syntaktischen und pragmatischen Rollen bilden jeweils eine Hierarchie in sich, welche miteinander korreliert. Üblicherweise bilden demnach Subjekt und Diskurs-Topik eine gemeinsame Einheit (sogenannte Topik-Subjekt-Persistenz) (Sanders/Gernsbacher 2004: 82).

\begin{tabular}{lccc}
\hline syntaktische Rolle & Subjekt & direktes Objekt & indirektes Objekt \\
semantische Rolle & Agens & Patiens & Rezipient \\
pragmatische Rolle & Diskurs-Topik & Paragraph-Topik/Comment & Satz-Topik/Comment \\
\hline
\end{tabular}

Tabelle 13: Korrelation von syntaktischer, semantischer und pragmatischer Rolle nach Givón 1983: 22 f.

In Sprachen, welche der Topik-Kommentar-Relation folgen - zum Beispiel die ObUgrischen Sprachen -, bleibt die Relation Topik-Subjekt bestehen, und die Diathese wird gegebenenfalls angepasst. Darüber hinaus hat die Topik-Kontinuität Auswirkungen auf die Wahl des anaphorischen Verweismittels.

Der folgende Abschnitt beschreibt die Auswirkungen der Topik-Subjekt-Persistenz auf die morpho-syntaktische Ebene und auf verschiedene Mechanismen, welche zur Beibehaltung dieser eingesetzt werden können (sogenanntes reference-tracking, Beschreibung und Definition im Anschluss) und geht auf die Rolle der Possessivkonstruktion dabei ein.

\subsubsection{Referenzlinguistik}

Ein wichtiger Faktor bei der Textherstellung ist, wie zuvor erwähnt, die Kohäsion, die grammatische Verknüpfung von Textkomponenten (verglichen die Kriterien der 
Textualität). Diese erfolgt auf Ebene der (Morpho-)Syntax und zwar in Form von Referenzbeziehungen, referentieller Bewegung und durch referentielle Verweismittel. Bei Hellwig 1984 wird daher beispielsweise auch von "referentieller Progression « gesprochen, bei Sanders/Gernsbacher 2004 hingegen von »referentieller Kohärenz« (»referential coherence« Sanders/Gernsbacher 2004: 80). Der letzte Begriff wird in der vorliegenden Arbeit vermieden, da es hier terminologische Überschneidungen mit der Kohärenz, welche einen anderen Aspekt der Textkontinuität bezeichnet, gibt. Die Referenz selbst ist sowohl ein Kriterium der Textanalyse (siehe Kapitel 3.2.1 Textlinguistik) als auch Gegenstand der Referenzlinguistik.

Referenz ist die »Bezugnahme des Sprechers auf außersprachliches mit sprachlichen und nicht-sprachlichen Mitteln « (Bußmann 2002: 554). Analog dazu ist der Referent das »Bezugsobjekt eines referierenden sprachlichen Ausdrucks« (Vater 2005: 69), auf welchen »mit sprachlichen Ausdrücken [...] Bezug genommen « wird (Bußmann 2002: 554). Das Phänomen der Referenz operiert auf mehreren linguistischen Ebenen, zum Beispiel im Bereich der Kognition, aber auch Syntax und Pragmatik haben mit Teilaspekten der Referenz zu tun (verglichen Vater 2005: 11), bei Consten 2004 wird Referenz so auch als »Identifikation eines Gegenstandes für den Hörer « bezeichnet (Consten 2004: 39). Hierbei werden zwei wichtige Arten von Referenz unterschieden, zum einen die Situationsreferenz als auch die Ding- (Vater 2005: 71) oder Gegenstandsreferenz. »Die Situationsreferenz ist die übergeordnete Referenzform: Ein Satz referiert gewöhnlich auf eine Situation« (Vater 2005: 71), während die Dingreferenz auf Personen/Gegenstände (Referenten), mithilfe von referentiellen Verweismitteln, welche je nach pragmatischer Eigenschaft des jeweiligen Referenten variieren (Lexikalische Ausdrücke, Nominalphrasen, Pronomen, Proformen), Bezug nimmt (verglichen Vater 2005: 71).

Ein Referent ist damit ein »Objekt, Ort, Eigenschaft oder Ereignis in der Realität, [auf welchen] mit sprachlichen Ausdrücken [im Text] Bezug genommen [wird] «(Bußmann 2002: 554). Demnach wird eine Entität der außersprachlichen Welt im Text bzw. in der sprachlichen Äußerung von einem Referenten repräsentiert, auf welchen mit sprachlichen Ausdrücken referiert, das heißt referentiell verwiesen wird. Beim referentiellen Verweisen werden zwei Hauptarten unterschieden: (ana-)phorisch und deiktisch. Dementsprechend verfügen Sprachen über deiktische und (ana-)phorische Verweismittel. In der vorliegenden Arbeit stehen diese sprachlichen Realisierungen von Referenz, die referentiellen Verweismittel, im Fokus, der Begriff Referenzlinguistik wird ausschließlich darauf bezogen.

Anaphorische Verweismittel oder Anaphern haben grundlegend die Funktion des Rück(ver)weisens (Kern 2010: 77). Damit ist in der Verwendung einer Anapher impliziert, dass der Referent, auf welchen verwiesen wird, bereits im Text erwähnt wurde (Lehmann 2013a). Die Anapher verfügt damit über einen Antezedens, zu welchem sie in einem asymmetrischen Verhältnis steht, "da die Anapher vom Antezedens abhängig ist und dieses wieder aufnimmt « (Kern 2010: 78). Das Antezedens, muss nicht zwangsläufig im selben Satz oder im unmittelbar vorangehenden Satz erscheinen, es muss lediglich im Text oder »in der betreffenden Äußerungssituation « auftreten (Vater 2005: 99). Wie weit die Referenz eines anaphorischen Verweismittels zurückreicht, hängt auch von der Salienz (»Zugänglichkeit«, »accessibility«) oder auch Aktiviertheit 
des Referenten ab. Antezedens und anaphorischer Ausdruck sind demnach koreferent, das heißt, sie verweisen auf denselben Referenten (Vater 2005: 18). Koreferenz kann auf verschiedene Weise erstellt werden, zum Beispiel durch Rekurrenz (Wiederholung des lexikalischen Ausdrucks oder Synonyme), aber auch durch Pronominalisierung (Verwendung von Pro-Formen und Pro-Elementen) (Gansel/Jürgens 2002: 36). Pronomen haben im Gegensatz zu Nomina keine eigene Referenz, das heißt, sie referieren nicht auf eine außersprachliche Entität, sondern verweisen innerhalb des Textes jeweils auf einen bestimmten (meist topikalen) Referenten:

Gerade hier wird die referentielle Abhängigkeit deutlich, da das Pronomen nicht über eine eigene virtuelle Referenz verfügt. Die Referenz kann erst aus dem Bezug zum Antezedens abgeleitet werden. (Kern 2010: 78)

Personalpronomina sind ebenfalls sogenannte Pro-Formen (Definition siehe Kapitel 1.2). Dabei werden zwei Kategorien unterteilt, sichtbare und verborgene, phonologisch leere (coverte) Pronomen. Coverte Pronomen werden als Leerstelle im Satz realisiert, da zusätzlich die in ihnen kodierte Person an einem anderen Satzglied markiert wird (verglichen Reichert 1986: 85).

Handelt es sich dabei um ein Verb, wird die Pro-Form als Personalmarker der objektiven Konjugation bezeichnet, handelt es sich um ein Nomen, spricht man vom Possessivsuffix. Die Konstruktionstypen 1.b.2 und 1.b.3 (siehe Tabelle 10) entsprechen damit einmal der Realisierung mit overtem und einmal mit verborgenem Pronomen. Neben den Pro-Formen existieren in den pro-drop-Sprachen ferner Pro- oder Null-Elemente, sogenannte Null-Morpheme. Null-Morpheme kodieren Referenten mit höchster Topikalität, da sie die kleinste morpho-phonologische Realisierung eines anaphorischen Verweiselementes, eine Leerstelle, darstellen:

Morphologisch nicht gekennzeichnete gramm. Bestimmung, die, um das System der sonst durch Affixe markierten Unterschiede im Flexionsparadigma in seiner Regelmäßigkeit zu bewahren, in der Form Null (ø) angenommen wird. (Bußmann 2002: 477)

Während einige Theorien alle Mechanismen unter der Anapher zusammenfassen, sprechen andere nur bei pronominalen Ausdrücken, welche sogenannte Platzhalterfunktionen haben, von Anaphern.

In linguistic terminology, the word [anaphora, G.J.] has two different analytic meanings: (a) it denotes a certain function, namely the activity of >re-ferring which is performed by means of a variety of word classes [...]; (b) it denotes a certain class of expressions, the so-called sthird-person pronouns«. (Ehlich 1982: 315)

Andere Theorien, zum Beispiel Brinker 2005, sprechen von expliziter Wiederaufnahme und beziehen alle Arten von Koreferenz mit ein (Brinker 2005: 27). Es ist auch möglich, vorgreifend auf einen Referenten zu verweisen, mit sogenannten Kataphern. Gemeinsam bilden Anapher und Katapher die endo-phorischen, das heißt textinternen Verweismittel (Lehmann 2013a).

Unter dem Begriff »Deixis«, griechisch für ,zeigen, hinweisen, wird ein »Prozess des Zeigens auf Größen der außersprachlichen Realität» verstanden (Vater 2005: 17, 
Anmerkung 9). Anaphorik und Deixis gemeinsam ist dabei, »durch Verweise in bestimmte Verweisdomänen einen Referenten zu identifizieren« (Consten 2004: 22).

Deictic expressions are related to a specific type of domain, the Zeigfeld (deictic field); in this regard they differ from all other words, which are related to the Symbolfeld (symbolic field). (Ehlich 1982: 324)

Ein sprachliches Mittel, welches deiktische Funktionen anzeigt, ist ein sogenanntes Deiktikum. Diese sind sprecherbezogen, das heißt, meist bildet der Sprecher selbst den Bezugspunkt, das sogenannte deiktische Zentrum (Origo), »mit jedem Sprecherwechsel wechselt das deiktische Zentrum und folglich das gesamte Bezugssystem« (Lehmann 2013b).

Dieses Bezugssystem unterscheidet die Deixis von der Anaphorik (Kern 2010: 78): Anaphern haben ihr Bezugselement (Antezedens) im Text, Deiktika bei den Sprechaktteilnehmern, mit anderen Worten, setzen Anaphern die Bekanntheit eines Referenten voraus, Deixis dessen Anwesenheit (Consten 2004: 5). Analog zu endo-phorischen, textinternen Verweismitteln spricht man bei der Deixis daher vom exo-phorischen Verweis (»Verweisen aus dem Text heraus« Vater 2005: 18, Anmerkung 10). Abgeleitet auf die Aktiviertheit eines Referenten erfolgt diese anaphorisch durch dessen Vorerwähntheit und deiktisch auf dessen Teilnahme am Diskurs. Die erste und zweite Person ist somit immer deiktisch und aktiviert, aber auch bei den dritten Personen kann von Deixis gesprochen werden, wenn diese Gegenstand des Diskurses ist (das heißt, das, worüber gesprochen wird) - statt einem Personalpronomen der dritten Person wird hier oft ein Demonstrativpronomen verwendet (Ehlich 1982: 321). Demonstrativpronomina verfügen über die »semantische Funktion des Verweises auf in der Situation Gegebenes bzw. auf Vorerwähntes « (Bußmann 2002: 152). Daher sprechen manche Theorien auch vom demonstrativen oder auch demonstrativ-deiktischen Gebrauch (Lehmann 2013b). Dabei muss der Referent jedoch nicht zwangsläufig in konkreter Form präsent sein; es reicht aus, wenn er den Sprechaktteilnehmern zum Beispiel durch die Diskurswelt, das heißt eine Erzählung, oder Ähnliches salient ist.

Somit sind Anaphorik und Deixis zwar prinzipiell als eigenständige Begriffe zu definieren, operieren jedoch in einem gemeinsamen Bereich und sind daher nicht immer klar voneinander abzugrenzen:

In fact, within linguistic theory, no clear-cut distinction between deixis and anaphora has to date been proposed. The terms are used not only conventionally as mere labels and analytically [...], but also to refer to partially overlapping sets of linguistic entities. (Ehlich 1982: 318)

Deutlich wird dies insbesondere innerhalb eines Textes. Auch hier kann deiktisch verwiesen werden, wenn auch an sich die Sprechsituation fehlt:

Texts, however, are characterized by the fact that they are removed from the speech situation [...]. Thus, the existence of deictic phenomena within texts [...] confronts the analyst with a problem as to the definition of Deixis itself. If categories taken from the speech situation are used, text deixis itself is a contradiction in terms. (Ehlich 1982: 318) 
Die vorliegende Arbeit verwendet aus diesem Grund den Begriff der Text-Deixis nach Ehlich 1982 (es existiert analog zur Doppelbezeichnung Text/Diskurslinguistik auch der Begriff Diskurs-Deixis, welcher hier jedoch nicht verwendet wird). Text-Deixis wird somit als Unterart der Deixis verstanden, welche wie jede andere Art der Deixis operiert.

Im Text wird dabei eine Verbindung zwischen der sprachlichen Einheit und dem Referenten anstatt einer real anwesenden Entität angezeigt (Diessel 2006: 475). Der Unterschied besteht also lediglich im Zeigefeld bzw. Verweisraum (»deictic space»), innerhalb dessen der deiktische Verweis erfolgt:

The text-deictic use of deictic expressions is to be seen as a subtype of the use of deixis in general. The deictic procedure in this case shares the properties of deictic procedures elsewhere. The difference between text deixis and other forms of deixis is [...] constituted by different Zeigfelder [...] or by different deictic spaces (Verweisräume), to which the deictic procedure is related. (Ehlich 1982: 331)

Sämtliche Mechanismen, welche in der vorliegenden Arbeit als deiktisch bezeichnet werden, sind somit als text-deiktisch zu verstehen, darunter fallen sowohl Verweise auf die ersten und zweiten Personen in einem Dialog innerhalb einer Erzählung als auch der Verweis auf einen der Sprechaktteilnehmer (da diese ja nur in der ursprünglichen mündlichen Überlieferung der Erzählung tatsächlich existent waren, für die verschriftlichte Variante genügt ein Leser). Dennoch sind solche Verweise mit in die Notation eingeflossen und somit Bestandteil des Korpus-Materials. Die personalpronominalen Verweise auf die dritten Personen (inklusive Possessivsuffixe) werden entweder als anaphorisch oder demonstrativ-deiktisch kategorisiert, Demonstrativpronomina ebenfalls als demonstrativ-deiktisch. Ob ein Possessivsuffix der dritten Person als anaphorisch oder text-deiktisch angesehen werden kann, unterliegt mehreren Faktoren und muss mit dem Kontext betrachtet werden. Dies wird bei der Vorstellung der einzelnen Mechanismen in Kapitel 5.2 noch thematisiert werden.

Im vorherigen Abschnitt wurden die zwei Hauptarten des Verweisens vorgestellt, anaphorisch und deiktisch. Wie der Abschnitt zur Text-Deixis gezeigt hat, kommen beide Verweisarten im Text vor und werden dort zur Strukturierung eingesetzt, das heißt, sie werden vom Sprecher zur Informations-Strukturierung eingesetzt. Abgeleitet auf die Thematische Progression im Text entspricht die Anaphorik dabei der »Anknüpfung an Bekanntes", die Deixis der »Neueinführung " (Consten 2004:5); mit anderen Worten, anaphorische Verweismittel werden häufig zur Kodierung topikaler Elemente verwendet, deiktische zur Markierung des Fokus einer Satzaussage. Man spricht auch von Beibehaltung (»sustention«) versus Fokussierung.

Die Beibehaltung entspricht dabei der referentiellen Kohärenz, welche mittels verschiedener Wiederaufnahmemechanismen erfolgt. Diese sind einzelsprachlich verschieden und bilden in ihrer Gesamtheit das System des reference-tracking einer Sprache, womit das Verfolgen eines topikalen Referenten im Textverlauf anhand der verwendeten anaphorischen Verweismittel bezeichnet wird (verglichen Kibrik 2001: 1124). ${ }^{64}$

64 Hier spricht man einerseits von den sog. primären referentiellen Verweismitteln wie den Anaphern sowie von den sog. untergeordneten Mechanismen, z. B. Satzstellung, Diathese (verglichen Kibrik 2001: 1125). 
Dabei existiert ein Zusammenhang zwischen der Wahl des anaphorischen Verweismittels, seiner morpho-phonologischen Größe und der Salienz des Referenten. Lambrecht 1994a unterteilt den Status eines Referenten in drei Arten: aktiv, inaktiv und salient (»active« - »inactive« - »accessible«, Kern 2010: 71). Ist der Referent wenig salient (»low accessible«), zum Beispiel ein bislang nicht erwähnter, neuer Referent, so werden größere morpho-phonologische Einheiten wie lexikalische Erwähnungen in Form von Nominalphrasen verwendet, um auf diese zu referieren. Dies erfolgt häufig zu Beginn eines Textes - insbesondere einer Erzählung - im sogenannten frame-setting, in welchem Ort und/oder Zeit der Handlung festgesetzt wird und die Partizipanten wie Hauptheld und gegebenenfalls dessen Antagonist eingeführt werden. Wird beispielsweise ein Paragraphen-Topik zu einem späteren Zeitpunkt in die Handlung eingeführt, so erscheint er dort für gewöhnlich ebenfalls als lexikalische Erwähnung. Ist der Referent hingegen salienter (»highly accessible«), beispielsweise im weiteren Verlauf der Handlung, sind kürzere morpho-phonologische Einheiten wie Pronomen ausreichend, um auf diesen Referenten zu verweisen (verglichen accessibility theorie nach Ariel 1999, zum Beispiel bei Sanders/Gernsbacher 2004: 79f.). Salienz wiederum korreliert mit der Topikalität - der Referent, welcher als Topik fungiert, ist automatisch der am höchsten aktivierte Referent und damit am salientesten. Auch hier nimmt die Aktiviertheit innerhalb der Hierarchie der topikalen Rollen vom Diskurs-Topik zum Satz-Topik hin ab. Die Korrelation von pragmatischer und syntaktischer Rolle betrifft daher auch das Paragraphen-Topik, das sehr oft als direktes Objekt realisiert wird (Givón 1983: 22). Die ob-ugrischen Sprachen verwenden in diesen Fällen ein eigenes Paradigma, um Paragraphen-Topiks in der Rolle des direkten Objekts am Verb zu kodieren: Die objektive Konjugation. Givón 1983 führt den Grad der Aktiviertheit des Topiks und die Sorten der anaphorischen Verweismittel in einer Skala zusammen, die einzelnen Bestandteile werden im Folgenden genauer beschreiben:

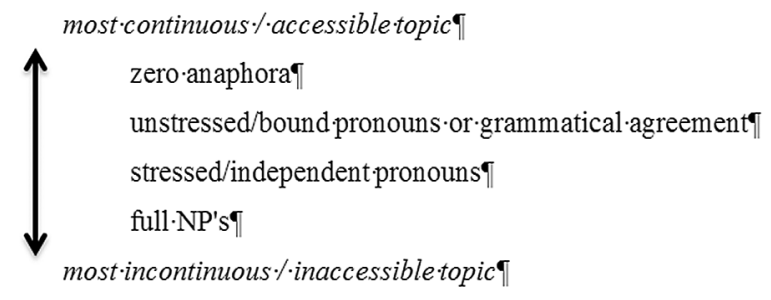

Abbildung 6: Skala Salienz ("accessibility") des Topik und Anaphorisches Verweismittel nach Givón 1983: $17 f$.

Des Weiteren ist neben der Topikalität der Referenten die Strukturierung des Textes bei der Wahl des anaphorischen Verweismittels ausschlaggebend. Erwähnungen im gleichen Abschnitt (Paragraphen) erfolgen mit Pronomen oder Nullanaphern, Erwähnungen desselben Referenten in einem anderen Paragraphen können zur Verwendung von Nominalphrasen führen (Fox 1996: i). Dies ist insbesondere bei der Re-Aktivierung der Fall, das heißt, wenn der Referent länger nicht Teil der Handlung und damit nicht aktiv war und dann wieder am Handlungsverlauf teilnimmt. 
Die kommunikative Funktion dieses Prinzips ist offensichtlich, [...] je weiter die Erwähnung des Referenten zurückliegt, desto mehr Aufwand ist zu treiben, um die Aufmerksamkeit wieder auf ihn zu richten. Insbesondere können in der Zwischenzeit ja auch andere Referenten genannt worden sein, von denen der gemeinte nun (durch lexikalische Mittel) unterschieden werden muß. (Lehmann 2013a)

Es spricht von ausgesprochen hoher Topikalität, wenn ein Referent über ParagraphenGrenzen hinaus mit Null-Morphemen wiederaufgenommen wird. Das System der Wiederaufnahme-Mechanismen ist somit äußerst komplex, die Größe eines Wiederaufnahme-Elementes kann auch davon abhängen, ob ein an sich schon bekannter, jedoch nicht genauer bestimmter Referent nun aufgrund des Handlungsverlaufs präzisiert wird (verglichen Vater 2005: 98). ${ }^{65}$

$\mathrm{Zu}$ Beginn des Kapitels wurde auf die Possessivkonstruktion als ReferenzpunktKonstruktion im Sinne der kognitiven Linguistik eingegangen. Aber auch die Beibehaltung bzw. das reference-tracking kann als eine Art der Referenzpunkt-Konstruktion angesehen werden, vielmehr sind sich die Funktionen, welche den topikalen Rollen bei der Textstrukturierung zugeschrieben werden, denen der Referenzpunkt-Konstruktionen sehr ähnlich: Sowohl Topik (und insbesondere das Diskurs-Topik) als auch Referenzpunkt dienen als Anker, um neue Information anzuknüpfen, welche dadurch salient wird:

A reference point is »a salient entity evoked for purposes of mentally accessing another " while a topic in cognitivism is considered to be a mental 'address' to which some notion is 'delivered' [...] for discourse purposes. (Langacker 1993: 24)

Somit sind nicht nur Possessivkonstruktionen in Referenzpunkt-Modellen zu beschreiben, sondern auch die Progression in einem Text. Hier fungiert das Topik als Referenzpunkt. Da es bekannt ist, eignet sich ein topikaler Referent daher als Referenzpunkt, an dem der Sprecher neue und eher unbekannte Information (das Comment) verankern und für den Hörer salienter werden lässt. Die neue Information ist demnach das Target, als dominion fungiert der Text selbst:

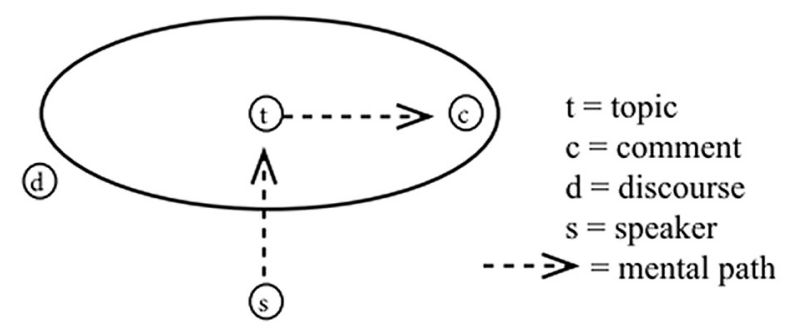

Abbildung 7: Das Referenzpunkt-Modell nach Langacker 1993 abgeleitet auf den Text. Eigene Adaption.

65 Eine ausführlichere Beschreibung des reference-tracking im Ob-Ugrischen anhand von Textbeispielen, welche ebenfalls in der Analyse der vorliegenden Arbeit Berücksichtigung gefunden haben, siehe z.B. Janda 2011 und Röll 2011. 
Deiktische Verweismittel hingegen dienen oft der Fokussierung. Auch hier korreliert die morpho-phonologische Größe der sprachlichen Einheit mit der Salienz des Referenten. Statt eines Null-Morphems kann ein Demonstrativpronomen auf einen Referenten verweisen. Ein lexikalisch erwähnter Referent kann ebenfalls mit einem Demonstrativpronomen zu einer größeren Nominalphrase erweitert werden, oder neue Information kann sogar in Form eines Relativsatzes dem Referenten hinzugefügt werden. Hierbei geht es um die Aufmerksamkeit des Hörers, welche vom Sprecher auf einen bestimmten Teil der Satzaussage gelenkt werden soll:

To communicate effectively, the hearer's attentiveness needs to be brought into concord with that of the speaker. I wish to use the term 'to focus' to denote this job of communication. One of the main means of focusing the attentiveness of the hearer is the deictic procedure. (Ehlich 1982: 325)

Wie auch Anaphorik und Deixis nicht gänzlich voneinander zu trennen sind (siehe vorangehende Beschreibung), existiert auch zwischen Fokussierung und Beibehaltung keine strikte Opposition (verglichen Ehlich 1982: 331). Beides kann auch kombinierend eingesetzt werden, zum Beispiel bei der Re-Aktivierung eines Referenten im Text. So können auch topikale Rollen fokussiert werden, beispielsweise mithilfe von emphatischen Personalpronomina. Es handelt sich also um ein Zusammenspiel beider Verfahren, was die Kommunikation, das heißt die Informations-Struktur, überhaupt ausmacht. Auch Verhältnis und Funktion von Anaphorik und Deixis lassen sich so charakterisieren:

It [the opposition, G.J.] also implies a certain affinity of the two members standing in contrast to each other. This also seem to be the case with deixis and anaphora. [...] Their characteristic in common is their linguistic function: directing a hearer's attention in the on-going process of communication. (Ehlich 1982: 331)

Einige Theorien, zum Beispiel Diessel 2006, sprechen daher von der Funktion der sogenannten "Joint Attention« (gemeinsame Aufmerksamkeit) in Zusammenhang mit der Verwendung deiktischer Verweismittel im Text. Insbesondere Demonstrativa, also Deiktika der dritten Person sind hier Forschungsgegenstand:

Demonstratives constitute a unique class of linguistic expressions serving one of the most fundamental functions in language: In their basic use, they serve to coordinate the interlocutors' joint focus of attention. (Diessel 2006: 464)

Unter dem Begriff Joint Attention wird ein komplexes linguistisches Phänomen zusammengefasst, welches sowohl Sprecher, Hörer und den Gesprächsgegenstand involviert; beide Sprechaktteilnehmer müssen ihre Aufmerksamkeit auf denselben Gesprächsgegenstand richten, um erfolgreich kommunizieren zu können (Diessel 2006: 465). Mit anderen Worten, das Topik muss dem Hörer bekannt und salient sein, der Sprecher muss seine Satzaussage dahingehend strukturieren. Dies gelingt dem Sprecher unter anderem dadurch, dass er mittels Demonstrativa den Hörer zum jeweiligen Fokus der Aufmerksamkeit dirigiert (verglichen Diessel 2006: 469), sodass ein gemeinsamer Kenntnisstand (shared knowledge) gegeben ist. Dabei kann die Aufmerksamkeit nicht nur auf neue Referenten gelenkt, sondern auch auf vorher erwähnte gerichtet werden oder zur Herausstellung eines Referenten aus einer Gruppe (Diessel 2006: 470). 
Deiktika, und insbesondere Demonstrativa, können somit zur Informations-Strukturierung eingesetzt werden, indem sie Referentenwechsel (Topic-Shifts) anzeigen und dabei die Aufmerksamkeit des Hörers auf diese lenken, das heißt, sie fokussieren den infrage kommenden Referenten (siehe dazu Kapitel 5.2).

Zusammenfassend lassen sich anaphorische Verweismittel interpretieren als Hinweis des Sprechers an den Hörer, die momentane Aufmerksamkeit beizubehalten (»sustain the previously obtained orientation of attention « (Ehlich 1982: 329), wohingegen deiktische Verweismittel als Anleitung vom Sprecher und den Hörer aufgefasst werden können, jetzt seine Aufmerksamkeit auf etwas anderes zu richten (verglichen Bosch 1983: 56). So können die Mechanismen zur Beibehaltung, Fokussierung und ReAktivierung gemeinsam im Sinne der Informations-Strukturierung zusammengefasst werden.

\subsection{Die Referentialität des Possessivsuffixes}

Der letzte Abschnitt des Kapitels befasst sich mit der Referentialität des Possessivsuffixes, das heißt, nun werden die referentiellen Eigenschaften der Possessivsuffixe anhand der zuvor beschriebenen theoretischen Aspekte vorgestellt. Der folgende Abschnitt enthält darüber hinaus einen Exkurs zur Definitheits-Theorie, dem Beschreibungsrahmen, welcher häufig für die sogenannten sekundären Funktionen des Possessivsuffixes herangezogen wird. Dort sollen die Analysekriterien der Definitheit vorgestellt und nach den referentiellen und text-linguistischen Aspekten, welche die theoretischen Grundlagen der vorliegenden Arbeit bilden, neu evaluiert werden.

Im Rahmen des reference-tracking wurden bislang nur solche Wiederaufnahme-Mechanismen vorgestellt, welche alle von der Koreferenz bzw. Referenzidentität bestimmt waren und damit dem Prinzip der Similarität (siehe hierzu auch Beschreibung der Wiederaufnahmemechanismen) entsprechen. Aber auch Kontiguität steht in gewisser Hinsicht mit der Anaphorik im Zusammenhang. Die sogenannte »implizite Wiederaufnahme« (Brinker 2005: 36) oder »indirekte, assoziative Anapher « (Vater 2005: 107) geht einher mit einem Referenzwechsel, das heißt, der Verweis auf einen Referenten erfolgt durch Mitverweis auf einen zweiten Referenten, welcher mit diesem in einer Beziehung steht (Gansel/Jürgens 2002: 36) und als anaphorischer Anker fungiert. Dies ist nur möglich, wenn dieser Referent topikal, das heißt aktiviert und bekannt ist (Consten 2004:68). Hierbei handelt es sich um nichts anderes als eine Referenzpunkt-Konstruktion, onomasiologisch als Possessivkonstruktion bezeichnet. Der Referent, welcher mit der Possessivkonstruktion aufgegriffen wird (das heißt das Target), kann zwar bisher im Text unerwähnt sein, erfährt aber durch die Verknüpfung mit dem Referenzpunkt selbst einen gewissen Grad an Salienz, wodurch der indirekte anaphorische Verweis entsteht.

Referenten, welche nicht identifizierbar sind, sind gleichzeitig inaktiv; umgekehrt ist sowohl ein aktiver als auch ein salienter Referent gleichzeitig identifizierbar. Die Einführung eines neuen Referenten über einen indirekten anaphorischen Verweis auf einen bereits bekannten, aktiven Referenten erleichtert den Mitteilungsprozess, indem nur ein Konzept neu eingeführt werden muss, durch welches Informationen in 
folgenden Äußerungen als gegeben oder seltener ableitbar erscheinen. Die wenigsten Äußerungen beinhalten ausschließlich neue Information. Sprecher tendieren somit dazu, »bereits evozierte Informationselemente beizubehalten, anstatt neue einzuführen « (Kern 2010: 72). Salienz beinhaltet dabei jedoch nicht nur Vorerwähntheit eines konkreten Referenten, sondern auch Weltwissen und den Handlungsverlauf des Diskurses an sich (verglichen Kern 2010: 73). Erkü/Gundel 1987 unterteilen die indirekte Anapher daher in drei Unterarten (»inclusive« - »exclusive « - »created «) (Erkü/Gundel 1987: 535), sodass ihre Beschreibung nicht nur solche Relationen miteinbeziehen kann, welche unter semantische Kontiguität fallen (die prototypischen Possessiv-Relationen wie Teil-Ganzes oder Verwandtschaftsbeziehungen), sondern auch solche Formen der indirekten Anapher, welche durch den Handlungsverlauf entstehen (»created«) (Erkü/ Gundel 1987: 535). Ferner können sowohl »inclusive« als auch »exclusive indirect anaphora sowohl durch »linguistische Faktoren, das heißt innerhalb des Textes, als auch durch außersprachliche Faktoren ausgelöst werden « (Erkü/Gundel 1987: 539). Dies wird im Hinblick auf die Textumgebung der Possessivkonstruktionen in Kapitel 5.2 noch thematisiert werden.

Bislang wurde diese Funktion der impliziten Wiederaufnahme generell als die Haupteigenschaft der Possessivkonstruktionen angesehen, in der Konsequenz wurde demnach von fast ausschließlich neuen, vorher unerwähnten Targets ausgegangen:

Possessive NPs introduce overwhelmingly new, previously unknown referents into the discourse by linking them to typically given possessor (PR) referents (Taylor 1996). (Willemse 2009: 13)

Das Target muss jedoch nicht zwangsläufig neu im Text sein, der Referenzpunkt kann auch zur Re-Aktivierung eines Referenten dienen. Es ist demnach notwendig, nicht nur die interne Verankerung innerhalb der Referenzpunkt-Konstruktion zu analysieren, sondern den gesamten Diskurs mit in die Analyse einzubeziehen, sowie das Erscheinen beider Referenten, welche als Referenzpunkt und Target fungieren, im gesamten Textverlauf:

This NP-internal identifying relation has to be distinguished from the external relations which the two discourse referents may maintain with other elements in the surrounding discourse. [...] The reference-point analysis thus offers a detailed account of the identification mechanism set up within possessive NPs. However, the PR (reference point) and PM (target) are themselves also discourse referents embedded in the discourse in which the possessive NP is used. (Willemse 2009: 17f.)

Hierbei geht es jedoch primär um die Eigenschaften der Possessivkonstruktion an sich, der Mechanismus der impliziten Wiederaufnahme - oder auch der Mechanismus der Referenzpunk-Konstruktion - erfolgt ungeachtet, ob ein morphologische Marker in der Konstruktion erscheint oder nicht. Somit kommt dem Possessivsuffix in der Konstruktion eine eigene Funktion zu und das Erscheinen unterliegt gewissen Voraussetzungen. Possessivsuffixe sind, wie eingangs erwähnt, sogenannte Pro-Formen und fallen daher ebenfalls unter die anaphorischen Verweismittel. In dieser Hinsicht ist das Possessivsuffix als morphologischer Marker des Referenzpunktes für den jeweiligen 
Referenten anzusehen, welcher den Kopf der Konstruktion bildet und diesen somit am Topik verankert und die Satzaussage strukturiert.

Während angenommen wird, dass das Erscheinen des Possessivsuffixes im Ungarischen obligatorisch ist (Forgács 2007: 137), so wird im Ob-Ugrischen die Topikalität des im Possessivsuffix kodierten Referenten vorausgesetzt (verglichen Nikolaeva 2002). Da die Analyse der vorliegenden Arbeit jedoch mehr darauf ausgerichtet ist, weshalb die Possessivkonstruktion mit Possessivsuffix im Text eingesetzt wird, und damit einhergehend, welche Referenten damit in Relation gesetzt bzw. kodiert werden, werden die Konstruktionen des Ungarischen und des Ob-Ugrischen gleichermaßen analysiert. Somit ist beispielsweise nicht allein ausschlaggebend, dass ein Possessivsuffix in der Konstruktion erscheint, sondern inwiefern der jeweilige Referent zusätzlich in der Konstruktion realisiert wird und wie weit die Verweiskraft des Possessivsuffixes jeweils zurückreicht. So kann die referentielle Verweiskraft des Possessivsuffixes den Referenzpunkt ausreichend kodieren, sodass dieser ansonsten mit einer Leerstelle kodiert wird (Konstruktionstyp b.3). Oder es handelt sich eher um Agreement mit dem als Pronomen oder lexikalisch realisierten Referenzpunkt innerhalb der Konstruktion (Konstruktionstyp b.2 und b.1). Hier ist außerdem zu beachten, wie sich die anderen Konstruktionen mit Possessivsuffix (und Verbalnomen bzw. Postposition als Kopf) einordnen und welche Auswirkungen dies auf die anaphorische Verweiskraft des Possessivsuffixes hat.

Darüber hinaus verfügen Possessivsuffixe über deiktische Verweiskraft. Da es sich bei den nominalen Personalmarkern in den ugrischen Sprachen um ein vollständiges Paradigma an Formen handelt, können auch die ersten und zweiten Personen kodiert werden. Personalmarker der ersten und zweiten Personen sind per se deiktisch, da sie einen der Sprechaktteilnehmer als Verweisdomäne heranziehen (verglichen Reichert 1986: 82), dies wird auch für Dialoge innerhalb eines Textes angenommen (verglichen Text-Deixis). Possessivsuffixe der ersten und zweiten Personen stellen somit eine Relation zwischen einem Referenten im Text und einem Sprechaktteilnehmer her, je nach Bezugspunkt (Sprecher oder Hörer) hat dies Auswirkungen auf das Target. Aber auch

die Possessivsuffixe der dritten Personen können für bestimmte Mechanismen im Sinne der Joint Attention eingesetzt werden.

Possessivsuffixe verfügen über sämtliche hier vorgestellten referentiellen Eigenschaften. Diese werden in unterschiedlicher Gewichtung je nach Suffix und Konstruktion zu verschiedenen text-strukturierenden Mechanismen eingesetzt, welche im empirischen Teil vorgestellt werden. Zuvor soll noch in einem Exkurs auf das Thema Definitheit im Zusammenhang mit Possessivsuffixen eingegangen werden, um die sogenannte sekundäre Funktion des Possessivsuffixes anhand der hier vorgestellten theoretischen Grundlagen zu re-analysieren.

\subsection{Exkurs: Evaluation des Ansatzes zur Definitheit}

Possessivkonstruktionen werden allgemein als definit beschrieben (Willemse 2009: 14). Hierbei muss jedoch detailliert betrachtet werden, was in diesem Kontext unter definit und unter Definitheit an sich überhaupt zu verstehen ist und auf welche Bestandteile 
der Possessivkonstruktion konkret sich die Bezeichnung »definit« bezieht. Ferner muss berücksichtigt werden, worin der Zusammenhang mit den Possessivsuffixen besteht, weswegen ihnen überhaupt eine sekundäre, definite Funktion attestiert wird. Der Exkurs an dieser Stelle ist dazu gedacht, auf diese Fragestellung genauer einzugehen und zu erläutern, weshalb die Interpretation einer Possessivkonstruktion als definite Nominalphrase durchaus als problematisch anzusehen ist (McGregor (Hg.) 2009: 2).

Unter Definitheit wird eine nominale Kategorie verstanden, welche die Grammatikalisierung von Identifizierbarkeit von Referenten anbelangt und häufig durch Artikel oder andere Determinantien ausgedrückt wird (Loos (Hg.) 2004f.).

Nach Hawkins 1978 gibt es folgende Arten von Definitheit (nach der Adaption von Vater 2005: 107f.):

a) Anaphorisch (»anaphoric use«):

Der Referent ist vorerwähnt und wird wiederaufgenommen; dies kann zum Beispiel durch einen definiten Artikel angezeigt werden.

b) Assoziativ-anaphorisch (»associative-anaphoric use«):

Hier wird eine Assoziation (meist in einer Teil-Ganzes-Beziehung begründet) zu einem bereits bekannten Referenten aufgebaut, durch die Assoziation wird der neue Referent salienter.

c) Deiktisch (»immediate situation use«):

Hier wird in der Gesprächssituation auf einen bestimmten Referenten verwiesen, welcher in der betreffenden Situation anwesend ist.

d) Abstrakt-situativ (»larger situation use«):

Ein Referent wird aufgrund von Weltwissen lokalisiert.

Der Definitheit zugrunde liegt die sogenannte Identifizierbarkeit (oder auch die »Lokalisierung eines Referenten in einer Menge von Referenten «Bußmann 2002: 147). Insbesondere in Artikelsprachen, wie es viele indo-europäische Sprachen sind, werden die Kategorie Definitheit und die Identifizierbarkeit von Referenten im Text synonym verstanden, und der definite Artikel als dessen prototypische formale Realisierung. Ähnlich wie ein definiter Artikel können auch Possessivkonstruktionen in gewisser Weise diese Art von Identifizierbarkeit erstellen, weswegen Definitheit und Possession durchaus in einem Zusammenhang stehen. Attributive Possessivkonstruktionen werden daher mit zu den Determinans-Phrasen (Bußmann 2002: 158) bzw. zu den definiten Nominalphrasen (Lyons 1999: 22) gerechnet. Determinans-Phrasen sind Nominalphrasen, welche durch ein Determinans - auch Determinierer - erweitert werden. Determinierer bestimmen ein Nomen näher (Bußmann 2002: 157), »semantisch gesehen ist Determination eine Abgrenzung des Referenten « (Vater 2005: 107). Zu den Determinierern zählen Artikel, Demonstrativa und bestimmte Pronomen wie Possessivpronomina und Interrogativpronomina (Brinker 2005: 29).

Die Identifizierungsmechanismen einer Possessivkonstruktion sind denen des definiten Artikels oder eines Demonstrativpronomens ähnlich, sodass viele Forschungsansätze (zum Beispiel Gerland 2014a, Simonenko 2014) die Kriterien nach Hawkins 1978 auch auf die Identifizierungsmechanismen von Possessivkonstruktionen ableiten: 
a) Anaphorisch (»anaphoric use»):

Der Referent (das Target) ist vorerwähnt und wird wiederaufgenommen. So steht diese Verwendung der Possessivkonstruktion ebenfalls in Opposition mit der Annahme, dass Possessivkonstruktionen vornehmlich neue Referenten einführen.

b) Assoziativ-anaphorisch ("associative-anaphoric use»):

Das bridging (Eigenschaft der indirekten, assoziativen Anapher, siehe implizite Wiederaufnahme), das heißt, die Bezugnahme auf einen neuen Referenten über dessen Assoziation zu einem bekannten Referenten, wobei der neue Referent mit dem definiten Artikel versehen wird, beruht ebenso auf Kontiguität wie Teil-GanzesRelationen, die wiederum zu den in Possessivkonstruktionen ausgedrückten KernRelationen zählen. Die Realisierung einer Aussage beispielsweise als, der Gipfel des Berges' oder ,sein Gipfel' kann zwar einzelsprachlich bestimmten Restriktionen, zum Beispiel der Animatheit oder Ähnlichem unterliegen, beide Realisierungen weisen jedoch Überschneidungen bei der Vorerwähntheit eines Referenten und der Verknüpfung eines neuen Referenten mit diesem auf. Ob nun der Referent, der

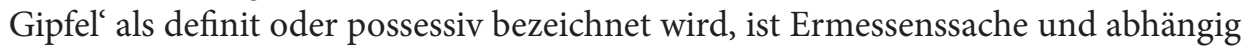
vom Inventar der Sprache und nicht von einer Opposition zwischen Definitheit und Possession.

c) Deiktisch (»immediate situation use«):

Diese Verwendung wird oft in Zusammenhang mit der Einzigartigkeit eines Referenten in der jeweiligen Äußerungssituation, zum Beispiel Anfang oder Ende einer Erzählung (Gerland 2014a: 276) oder mit der Kombination mit einem Demonstrativpronomen (Simonenko 20014; 4), gesetzt. Beide Erklärungsansätze sind meines Erachtens problematisch und deuten darauf hin, dass nicht alle für die Definitheit ausgearbeiteten Kriterien auf Possessivkonstruktionen eins zu eins übertragbar sein müssen. Nicht von der Hand zu weisen ist jedoch im Ansatz von Vater 2005 die Parallele zur deiktischen Verwendung. Wie im theoretischen Rahmen der vorliegenden Arbeit beschrieben ist hier meines Erachtens der Ansatz der Joint Attention zielführender als der des »immediate situation use«.

d) Abstrakt-situativ (»larger situation use»):

Diese Kategorie ist die meines Erachtens meist problematische. Die Kategorie des »larger situation use - oder zum Beispiel auch als "global uniqueness « bezeichnet, (Simonenko 2014: 4) - wird dann als Erklärungsansatz herangezogen, wenn die Relation nicht als prototypisch possessiv interpretiert werden kann und demnach kein Possessor zugeordnet werden kann. Auffallend ist dabei auch, dass es sich hier um ein ganz bestimmtes meist aus denselben Quellen zitiertes Set von Daten handelt, welches im Großteil der Studien als Begründung einer zweiten, nicht-possessiven, definiten Funktion des Possessivsuffixes herangezogen wird, zum Beispiel:

Apart from this function as possessive markers, the possessive suffixes of some Uralic languages have another main function: they mark definiteness in the same way as definite articles in Indo-European languages [...].In (4) [Beispiel (19) GJ] the possessive suffix is attached to a noun which refers to a unique entity. The earth is (without a special context) not possessed and according to Wagner-Nagy (2002) the possessive suffix is clearly interpreted as a marker of definiteness. In (5) [Beispiel (20) GJ] forest and snow are realized with the possessive suffix of the 
3rd person and again no possessor for either of them is mentioned in the context (and again only a special context would allow an interpretation where at least snow is possessed [...]). Thus, the interpretation of the suffix is that of a definiteness marker. (Gerland 2014a: 271).

(19) Nganasanisch, Gerland 2014a: $271^{66}$

$\begin{array}{lllll}\text { Мәu } & -\delta u & \text { sü̈rü } & \text { nil'ənu } & \text { čiiməə } \\ \text { earth } & -3 S G & \text { snow.GEN } & \text { under } & \text { hidden.PTPASS.3SG }\end{array}$

'The earth is covered with snow.'

(20) NM_text_008_0036 ${ }^{67}$

xum tujt $-e$ ja:nk $-e$ paryalt $-i$

man snow $-S G<3 S G$ ice $-S G<3 S G$ shake off $-P R S[3 S G]$

'The man is cleaning off the snow and ice.'

Es ist sicherlich nicht per se auszuschließen, dass es sich hier um eine bestimmte Funktion des Possessivsuffixes in Kombination mit einer bestimmten Wortgruppe handelt, allerdings fehlt diesen Belegen der Kontext, und es ist nicht möglich, Aussagen über die Häufigkeit dieser Vorkommen zu treffen. Im Korpus zur vorliegenden Arbeit beispielsweise finden sich kaum Belege, welche hiermit zu vergleichen wären. Meines Erachtens bedarf dieser spezielle Aspekt weiterer Erforschung und gezielter Elizitierung, sodass die vorliegende Arbeit von weiteren Erklärungsansätzen absieht.

Die Identifizierungsmechanismen von Definitheit und Possession sind sich somit ähnlich (Aikhenvald/Dixon (Hg.) 2012: 44), können jedoch keinesfalls gleichgesetzt werden. Hinzu kommt, dass in den ugrischen Sprachen die Determinantien nicht komplementär verteilt sind wie in manchen indo-europäischen Sprachen.

Während sich zum Beispiel im Deutschen definiter Artikel und Possessivmarker gegenseitig ausschließen, ist im Ungarischen diese Kombination nicht nur möglich, sondern der definite Artikel häufiger Bestandteil der Possessivkonstruktion ${ }^{68}$ (verglichen Molnár 2014: 59f.):

(21) HU 0154

$\begin{array}{llll}a & \text { zseb } & -\dot{e} & -b o ̈ l \\ \text { the } & \text { pocket } & -\mathrm{SG}<3 \mathrm{SG} & -\mathrm{ABL}\end{array}$

'Aus der Tasche.'/'Aus seiner Tasche.'/*'Aus seiner der Tasche.'

Somit hat der definite Artikel des Ungarischen nicht dieselben Eigenschaften wie der des Deutschen oder ist aufgrund seiner sekundären Entwicklung nicht ganz so restriktiv wie bei einer Artikelsprache. Doch auch die Kombination Demonstrativpronomen und Possessivmarker ist in den ugrischen Sprachen nicht komplementär:

$66 \mathrm{Da}$ ohne den Kontext keine vergleichbaren Belege aus dem Korpus der vorliegenden Arbeit aus dem Ob-Ugrischen herangezogen werden können, wird hier das Original-Beispiel aus der Quelle zitiert. Notation und Glossierung wie in der Quelle.

67 Hier wurde ein vergleichbares Beispiel mit dem Lemma ,Schnee' aus dem Korpus der vorliegenden Arbeit gewählt. Basierend auf den alternativen Analysevorschlägen in Kapitel 5.2. der vorliegenden Arbeit würde dieses Beispiel nicht als »kein Referenzpunkt zuzuordnen« klassifiziert werden. Über das Beispiel aus dem Komi in der Quelle kann aufgrund des fehlenden Kontextes keine weitere Aussage getroffen werden.

$68 \mathrm{Im}$ Korpus verfügen z. B. 554 von insgesamt 1.015 Vorkommen der Konstruktion Su Bs-Px im Ungarischen über einen definiten Artikel. 


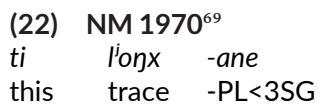

'Diese Spur.'/'Seine Spur.'/*'Diese seine Spur.'

Morphologische Marker in Possessivkonstruktionen werden ebenfalls generell mit Markierung von Definitheit in Zusammenhang gebracht, insbesondere pronominale Marker wie Possessivsuffixe (Aikhenvald/Dixon (Hg.) 2012: 44). Possessivpronomina erfüllen beispielsweise in diesen Konstruktionen die Kriterien der Determination (durch die Verwendung der ersten und zweiten Personen, welche das Possessum mit den Sprechaktteilnehmern verknüpft) (Lehmann 2013b) und Anaphorik (in ihrer Eigenschaft als Pro-Formen); dasselbe gilt in diesem Zusammenhang für Possessivsuffixe (verglichen Lyons 1999: 124f.). Tatsächlich ist das Possessivsuffix der dritten Person Singular Einbesitz das am meisten analysierte Possessivsuffix im Zusammenhang mit der Definitheits-Theorie, auf das der zweiten Person Singular Einbesitz wird meist in einem Nebensatz verwiesen, andere Possessivsuffixe werden selten thematisiert. Dies ist meines Erachtens zwei Umständen geschuldet, welche möglicherweise die Eigenschaften des gesamten Paradigmas des nominalen Personalmarkers verzerrt. Zunächst basiert die Annahme der sekundären Funktionen auf dem Nicht-Vorhandensein eines möglichen Possessors. Prototypische Possessivkonstruktionen sind solche, denen ein Possessor zugeschrieben werden kann und welche sich zu prädikativen Habeo-Konstruktionen nach dem Muster Possessor $=$ Subjekt und Possessum = direktes Objekt umwandeln lassen, was bei den nicht-prototypischen Konstruktionen zu ungrammatischen Ausdrücken führt (Kenesei/Vago/Fenyvesi (Hg.) 1998: 160).

Der Wegfall eines Referenten suggeriert dabei offensichtlich aber auch einen Wegfall der im Suffix kodierten Person. Dazu kommt die Fokussierung auf den Kopf der Konstruktion - dem determinierten Referenten in einer Determinans-Phrase entspricht der Kopf der Possessivkonstruktion, dem definiten Artikel analog dazu das Possessivsuffix; der Modifizierer selbst, welcher im Possessivsuffix kodiert ist, gerät aus dem Fokus der Analyse.

Ein definiter Artikel ist als Markierung aufzufassen, dass dem Hörer ein bestimmter Referent bekannt ist, der Artikel selbst ruft dabei keine Identifizierbarkeit hervor:

Der Artikel schafft weder Bekanntheit noch Unbekanntheit; er ist lediglich ein Signal für den Hörer (Leser), daß der Sprecher (Autor) bestimmte Informationen beim Hörer (Leser) als bekannt oder nicht bekannt voraussetzt. Die vorausgesetzten Informationen können inner- und/oder außertextlicher Art sein. (Brinker 2005: 30)

Das Possessivsuffix hingegen kodiert einen Referenzpunkt, an welchem der Referent (Target) verankert wird. Dieser Referenzpunkt ist vorerwähnt, das Target wird mit einer Pro-Form markiert, welche auf einen Referenten im Text oder die Sprechaktteilnehmer verweist. Aufgrund der Referentialität hat dies zur Folge: Das Target wird

69 Die Kombination von Demonstrativpronomen und Possessivpronomen ist im Deutschen nicht völlig ungrammatisch (im Gegensatz z. B. zu *die seine Spur'), aber sehr markiert und keinesfalls als neutrale Satzaussage aufzufassen. 
zum Beispiel salienter für den Hörer, rückt in den Fokus der Aufmerksamkeit, wird dabei re-aktiviert oder die Topik-Kontinuität bleibt - durch die Verknüpfung mit dem Referenzpunkt - erhalten. Die vorliegende Arbeit schlägt statt der Gleichsetzung der Kriterien von Hawkins 1978 mit den Funktionen der Definitheit die folgende Einteilung basierend auf der anaphorisch und deiktischen Verweiskraft der Possessivsuffixe folgende Zuordnung vor:

a. Anaphorisch Vorerwähntheit: Rückverweis auf einen vorerwähnten Referenten, zum Beispiel in der Konstruktion PosтP-Px im Ungarischen (siehe Kapitel 5.1.3);

b. Assoziativ-anaphorisch Salienz: Verankerung eines Targets am Referenzpunkt, zum Beispiel in der Konstruktion Subs-Px;

c. Deiktisch $\sim$ Joint Attention: Verweis auf einen Referenten in einer konkreten Äußerungssituation (text-deiktisch oder mit Verknüpfung mit einem Sprechaktteilnehmer $)^{70}$;

d. Abstrakt-situativ Einzigartikeit?

Die Identifizierungsmechanismen des Possessivsuffixes sind dadurch komplexer als es ein Vergleich mit dem definiten Artikel erfassen kann und müssen in einer eigenen Analyse beschrieben werden.

Possessivsuffixe scheinen in Zusammenhang mit der Referentialität komplexe Eigenschaften zu besitzen: Ihre Funktion ist nicht ausschließlich die formale Markierung eines Besitzverhältnisses innerhalb einer Possessivkonstruktion. Aus diesem Grund werden in der vorliegenden Arbeit die Vorkommen von Possessivsuffixen in Zusammenhang mit den im Possessivsuffixe kodierten Referenten und deren syntaktischen und pragmatischen Rollen im Text analysiert. Die formale Markierung erfolgt mittels Possessivsuffixen dergestalt, dass ein Referent kodiert wird, und je nach Person des kodierten Referenten wird dabei anaphorische oder deiktische Referenz aufgebaut. Die Bestandteile der Konstruktion, Referenzpunkt und Target können neben ihren Rollen als Modifizierer und Kopf innerhalb der Possessivkonstruktion gleichzeitig auch syntaktische (Kern)Rollen im Satz übernehmen. Als letzte Schicht ist dann die Informations-Struktur anzumerken, wie der Sprecher diese Gesamtheit an Mechanismen einsetzt, um dem Hörer die gewünschte Information zu übermitteln. All diese Aspekte werden für die Analyse im empirischen Teil der vorliegenden Arbeit herangezogen, welcher mit dem folgenden Kapitel beginnt.

70 Genau dieser Aspekt der Kodierung der ersten und zweiten Person entfällt in der Definitheits-Theorie weitestgehend. 



\section{Daten und Methodik}

Die Analyse der vorliegenden Arbeit ist text-linguistisch, korpus-basiert und semasiologisch ausgerichtet. Es wird daher nicht der Ausdruck von Possession mithilfe von Possessivsuffixen untersucht, sondern sämtliche Vorkommen (Token) von Possessivsuffixen werden berücksichtigt und anhand ihrer Konstruktionsart (Wortart des Kopfes), ihres Konstruktionstyps (Realisierung des Modifizierers) und ihrer Referentialität (Anzahl und Art der kodierten Person(en), letzte Vorerwähnung) klassifiziert (Type). Ziel der Analyse ist der Versuch einer Kategorisierung des Possessivsuffixes anhand der Konstruktionsart und ihrer Auswirkung auf die Strukturierung des Textes und damit eine Re-Analyse der possessiven und nicht-possessiven Funktionen des nominalen Personalmarkers. Gleichzeitig werden die Verwendungen in den einzelnen Sprachen miteinander verglichen.

\subsection{Daten und Quellen 4.1.1 Ob-Ugrisch}

Der Sprachvergleich der ugrischen Sprachen verläuft hauptsächlich synchron; die diachrone Betrachtung gestaltet sich besonders in den ob-ugrischen Sprachen als schwierig, da das zugängliche ob-ugrische Sprachmaterial lediglich etwas mehr als hundert Jahre zurückreicht. Wie eingangs erwähnt handelt es sich bei den ob-ugrischen Sprachen um bedrohte Minderheitensprachen mit verhältnismäßig geringer Dokumentation und einer relativ gering entwickelten Schreibtradition (siehe Kapitel 1.1). Dies hat auch Auswirkungen auf die Zugänglichkeit und Verarbeitung von Sprachmaterial. So ist es erst seit jüngerer Zeit möglich, auf annotierte und digitalisierte Korpora für die Analyse der ob-ugrischen Sprachen zurückzugreifen. Eine Vorreiterrolle spielt dabei das EuroBABEL Projekt »Ob-Ugrische Sprachen: konzeptionelle Strukturen, Lexikon, Konstruktionen, Kategorien " $(\mathrm{OUL})^{71}$, ein internationales Forschungsprojekt im Rahmen des EUROCORES Programm der European Science Foundation. Von August 2009 bis Juli 2012 ist in Zusammenarbeit mit fünf Universitäten (LMU München, Universität von Wien, Universität Helsinki, Eötvös Loránd Universität Budapest und der Universität Szeged) eine virtuelle Forschungs-Plattform (»virtual research environment») entstanden, welche auch ein zahlreiche zusammenhängende Texte aus vier ob-ugrischen Dialekten umfassendes, digitales, durchsuchbares Korpus beinhaltet. Im Rahmen des Nachfolgeprojektes »Ob-Ugrische Datenbanken: Analysierte Korpora und Wörterbücher kaum beschriebener ob-ugrischer Dialekte $(\mathrm{OUDB})^{72}$ wurde das digitale Korpus in den Jahren 2014 bis 2017 um den Jugan-Dialekt des Chantischen (Ostgruppe, Subdialekt des Surgut-Chantischen) und um das West-Mansische (in den Varianten Pelym, Vagilsk, Mittlere und unter Lozva) erweitert. Weitere umfangreiche Projekte zur Digitalisierung

71 Ob-Ugric Database http://www.oudb.gwi.uni-muenchen.de/index.php?abfrage=welcome_eurobabel\& navi=about\&subnavi= (Zugriff vom 14.05.2017).

72 Ob-Ugric Database, http://www.oudb.gwi.uni-muenchen.de/index.php?abfrage=welcome_eurobabel\& navi=about\&subnavi (Zugriff vom 14.05.2017). 
und linguistischen Dokumentation ob-ugrischer Sprachen sind das Projekt »Typologie der Negation in den obugrischen und samojedischen Sprachen « (NOS) ${ }^{73}$ der Universität Wien, das Projekt »Multimedia documentation of the endangered Vasyugan and Alexandrovo Khanty dialects of Tomsk region in Siberia ${ }^{74}$ von Andrey Filchenko sowie das Projekt »The Endangered Languages and Cultures of Siberia ${ }^{75}$ von Irina Nikolaeva.

Der Großteil des ob-ugrischen Textmaterials der vorliegenden Arbeit entstammt dem Korpus des OUL Projektes, welches neben der relativ umfangreichen Datenmenge den Vorteil besitzt, dass die Texte nicht unter einem spezifischen Forschungsaspekt (zum Beispiel der Negation) ausgewählt und aufbereitet wurden, sondern die Digitalisierung und Annotation für zukünftige linguistische Analysen im Vordergrund stand und somit ein heterogenes Korpus größtenteils bislang unveröffentlichten Materials entstanden ist. Das gesamte Korpus wurde im Internationalen Phonetischen Alphabet (IPA) transkribiert bzw. einheitlich transliteriert, worin es sich von bisherigen Textsammlungen zum Ob-Ugrischen unterscheidet, welche größtenteils in der Finnisch-Ugrischen Transkription, einer Variante der jeweiligen kyrillischen Verschriftlichung oder in einem eigenen Notationssystem des jeweiligen Forschers notiert wurden. Durch die Mitarbeit der Verfasserin im Projekt OUL sind insbesondere bei der Glossierung Aspekte mit eingeflossen, welche die Aufbereitung des Materials in der vorliegenden Arbeit insofern erleichtern, als das annotierte Material ohne größere Aufarbeitung zur Analyse bereitstand. Eine Transkription oder Um-Glossierung, beispielsweise der Possessivsuffixe, war somit nicht nötig. ${ }^{76}$ Auch das Textgenre, welchem der Großteil der Texte angehört, Erzählungen und Märchen, ist bestens für die in der vorliegenden Arbeit anvisierte Analyse geeignet. Es handelt sich dabei um fortlaufende Texte, die eine besondere Erzähltradition der Ob-Ugrier repräsentieren. Diese Erzählungen berichten typischerweise vom Abenteuer eines Haupthelden (zum Beispiel dem nord-mansischen E: $k^{w} a$ Pifris ${ }^{j}$ oder dem Surgut-Chantischen Pi:tiankali und dessen Schwester), oft tritt auch eine Antagonist, zum Beispiel der Mcyk i.ki im Surgut-Chantischen, auf. Die Handlungen sind somit fortlaufend aufeinander aufgebaut, die Zahl der Partizipanten ist relativ begrenzt, und meist ist die Rolle des Diskurs-Topik während des gesamten Textes fest etabliert, was beim reference-tracking deutlich erkennbar wird. Einige Texte sind Artikel aus den obugrischen Publikationsorganen Хӑнты Ясау und Луйма̄ Сэрипос.

Vervollständigt wird das Material von Belegen aus der Feldforschung, welche der Verfasserin aus der privaten Sammlung von Zsófia Schön zur Verfügung gestellt worden sind. Es handelt sich dabei um Transkriptionen von Belegen, welche für die eigene Forschung von Frau Schön elizitiert wurden und in einer MySQL-Datenbank analysiert und zusammengefasst sind. ${ }^{77} \mathrm{Da}$ es sich dabei um einzelne isolierte Sätze handelt, wurden

73 Typologie der Negation in den obugrischen und samojedischen Sprachen, http://www.univie.ac.at/negation/index.html (Zugriff vom 14.05.22017).

74 Multimedia documentation of the endangered Vasyugan and Alexandrovo Khanty dialects of Tomsk region in Siberia, http://www.policy.hu/filtchenko/FTG\%20ELDP\%20project/langdata.htm (Zugriff vom 14.05.2017).

75 The Endangered Languages and Cultures of Siberia, http://larkpie.net/siberianlanguages/ (Zugriff vom 14.05.2017).

76 Das Projekt »The Endangered Languages and Cultures of Siberia« benutzt beispielsweise die traditionelle Finnisch-Ugrische Transkription; zur Problematik der Glossierung siehe Kapitel 4.2.1.

77 Siehe dazu Schön 2014. 
sie gesondert analysiert, und auch der Umfang ist relativ gering. Dennoch sollen die Belege nicht völlig unerwähnt bleiben, da sie beispielhaft sind für die Verwendung von Possessivsuffixen in der unmittelbaren Sprechsituation und in der Interaktion mit den Sprechaktteilnehmern.

\subsubsection{Ungarisch}

Das Korpusmaterial des Ungarischen wurde analog zum vorhandenen ob-ugrischen Material eigens ausgewählt und vorbereitet, den ob-ugrischen Erzählungen entsprechen dabei ungarische Volksmärchen aus den Sammlungen von Károly Török (in Arany/Gyulai (Hg.) 1872) und László Merényi (Merény 1861), beide Sammlungen sind in digitalisierter Form entweder über das Projekt Gutenberg ${ }^{78}$ oder die Ungarische Elektronische Bibliothek ${ }^{79}$ zugänglich. Dazu kommen Kurzgeschichten neueren Datums aus dem Sammelband von Christina Kunze (Kunze (Hg.) 1999). Als Gegenstück zu den Zeitungsartikeln wurden EU-Nachrichtentexte aus dem Online-Verzeichnis der Pressemitteilungen der Europäischen Union ${ }^{80}$ gewählt. Sämtliches Material war in einer deutschen Übersetzung verfügbar, jedoch nicht annotiert. Dies musste im Vorfeld der Analyse erfolgen. Es existiert für das Ungarische auch ein annotiertes Nationalkorpus, dessen Aufbau und Abfragemaske jedoch nicht uneingeschränkt für die Zwecke der vorliegenden Arbeit eingesetzt werden konnte. Das Ungarische Nationalkorpus (HNC) besteht derzeit aus rund 188 Millionen Wörtern. Diese verteilen sich auf jeweils fünf Sub-Korpora, einmal dialektal und einmal nach Textgenre. Es ist möglich, sowohl nach einem Lemma (»stem») als auch nach einer Kombination aus zwei Lemmata zu suchen, deren relative Position zueinander ebenfalls in der Suchanfrage in gewissem Umfang berücksichtigt werden kann. Dasselbe gilt für Wortformen; so lässt sich beispielsweise gezielt nach der Form házamban ,Haus-SG $<1 \mathrm{SG}-\mathrm{INE}^{\mathrm{c} 1}$ oder a házamban ,DEF Haus-SG $<1 \mathrm{SG}-\mathrm{INE}$ `suchen.

Es ist auch möglich, nach dem Lemma ház ,Haus' in Verbindung mit zusätzlichen Merkmalen wie »number: singular > possessive: yes $>$ poss. number: singular $>$ poss. person: first > pluralizer: no > anaphoric: no $^{82}>$ case: $-\mathrm{bAn}$ « zu suchen (die Parameter der Suche nach házamban). Die Annotation beinhaltet die Wortart sowie verschiedene morphosyntaktische Merkmale, zusammengefasst als »MSD-Code«. Dieser erscheint entweder als Information am gesuchten Lemma oder ist selbst als Suchanfrage abrufbar. So kann gezielt nach Formen mit dem Possessivsuffix der ersten Person Singular gesucht werden (MSD-Code »PSel«), zum Beispiel um sämtliche Allomorphe des Suffixes zu erhalten. Die Anzahl der Suchergebnisse kann beschränkt werden (wenn man beispielsweise 20 oder auch 50 Belege angezeigt bekommen möchte). Der Kontext des Suchergebnisses kann ebenfalls bestimmt werden. Hier ist es möglich, sich den gesamten

78 Projekt Gutenberg, http://www.gutenberg.org/wiki/Main_Page (Zugriff vom 14.05.2017).

79 Ungarische Elektronische Bibliothek (mek), http://nbn.urn.hu/N2L?urn:nbn:hu-2 (Zugriff vom 14.05.2017).

80 Europäische Union, http://europa.eu/rapid/search.htm (Zugriff vom 14.05.2017).

81 Zur Glossierung siehe Kapitel 4.2.1.

82 Dies schließt den Possessivmarker -é aus der Suche aus. 
dem Beleg vorangehenden Absatz (»with 1 paragraph context«) mit anzeigen zu lassen. Allerdings hat sich bei der Benutzung gezeigt, dass dies für die Analysezwecke der vorliegenden Arbeit nicht ausreicht, da der gesamte Text für die Kohäsion benötigt wird. Auch ist es nicht möglich, sich einen bestimmten Text anzeigen zu lassen. So ließe sich zum Beispiel eine Aussage treffen, dass eine bestimmte Form des Possessivsuffixes besonders häufig auftritt oder sehr selten vorkommt. Wenn man jedoch stattdessen nur gezielt nach der bestimmten Form suchen kann, erhält man zwangsläufig nur Texte, in welchen diese Form auftaucht und verfälscht damit möglicherweise den Eindruck. Es wird zu jedem Suchergebnis eine Angabe mitgeliefert, wie oft der Beleg insgesamt sowie pro einer Millionen Worte auftritt, was als Richtwert zwar hilfreich ist, jedoch meines Erachtens nicht eine textgestützte Analyse der Vorkommen ersetzen kann. Das HNC wurde daher zur gezielten Elizitierung in solchen Fällen herangezogen, in denen das Textkorpus keine (repräsentativen) Daten liefern konnte. Die Angaben dienen zu Vergleichszwecken, um das limitierte Korpus zu unterstützen, und wurden gesondert analysiert. Die Notation der ungarischen Texte entspricht der heutigen ungarischen Rechtschreibung, archaische Schreibweisen - zum Beispiel herczeg herzeg, Prinz ${ }^{c}$ - wurden aus den Quellen übernommen und mit einem entsprechenden Vermerk versehen.

Angesichts der geringen vorliegenden Textmenge - einerseits durch die Datenlage im Ob-Ugrischen, andererseits durch die erforderlichen zeitaufwendigen Vorarbeiten bei der Annotation des ungarischen Materials - ist dennoch eine geeignete Auswahl an Material vorhanden, insbesondere da es sich nicht nur um einzelne Textfragmente handeln sollte, sondern zusammenhängende Erzählungen nötig sind, um die referentiellen Eigenschaften der Possessivsuffixe erkennen und analysieren zu können.

Selbstverständlich repräsentiert das vorliegende Korpus auch nur einen Ausschnitt aller drei Sprachen, es konnten nicht sämtliche Dialekte berücksichtigt werden, und insbesondere das relativ spezifische Textgenre sowie das Alter mancher Belege (die ungarischen Märchentexte und die Materialien aus dem aus dem Feldforschungs-Archiv von Valerij Nikolajevič Černecov stammen vom Ende des 19. und Beginn des 20. Jahrhunderts) schränkt die Allgemeingültigkeit der hier formulierten Ergebnisse erheblich ein. Selbst wenn jedoch einige der Mechanismen im heutigen, alltäglichen Sprachgebrauch nicht mehr verwendet werden sollten, so sind die Belege dennoch als feste Formeln in der Erzähltradition zu werten, welche in dieser Form bis heute überdauern konnten und einen Einblick in das komplexe Verwendungsspektrum der Possessivsuffixe geben können. Dadurch bilden sie einen wichtigen Bestandteil für die Re-Analyse der Possessivsuffixe in den ugrischen Sprachen. Die in der vorliegenden Arbeit geleistete Vorarbeit und formulierten Ergebnisse können darüber hinaus als Ausgangspunkt weiterer Forschung dienen, welche beispielsweise andere Dialekte aus den vorhandenen digitalen Textkorpora und andere Textgenre miteinbeziehen. Abschließend sei noch die Sekundärliteratur in Form von Wörterbüchern erwähnt, welche zur Überprüfung herangezogen wurden. Für das Ungarische wurde mit den Wörterbüchern Hessky (Hg.) 2002 und Halász/ Földes/Uzonyi (Hg.) (2006) gearbeitet, und um feste Redewendungen ausschließen zu können (siehe Übersicht der festen Phrasen und Redewendungen), wurde zusätzlich ein Wörterbuch der Sprichwörter und Redewendungen (Paczolay 1991) hinzugezogen. Für das Mansische wurden Munkácsi/Kálmán 1986 sowie Баландин/Вахрушева (Нg.) 1958 herangezogen, für das Chantische das DEWOS/Steinitz 1966-1993. 


\subsection{Methodik}

Die Korpusbelege entstammen insgesamt fünfzig Texten, jeweils zehn ungarischen und Kazym-Chantischen, 13 Texten aus dem Surgut-Chantischen und siebzehn aus dem Mansischen. Auch hier liegt der Grund in Auswahl und Anzahl in der Zugänglichkeit; es sollten so viele Texte wie möglich berücksichtigt werden, jedoch wurde dabei stets die gleichmäßige Verteilung der Token zwischen den drei Sprachen im Auge behalten. Da die Texte zudem unterschiedlicher Länge sind, kann keine Aussage über eine Relation zwischen Textanzahl und Anzahl der Token getroffen werden, jedoch über die Anzahl der possessivsuffigierten Formen gegenüber der Gesamtanzahl an Wortformen. Auch die Anzahl der Token in Relation zur Anzahl der Sätze kann nicht ohne Weiteres bestimmt werden, da nicht jeder Satz mit einem Satzzeichen endet und umgekehrt nicht jedes Satzzeichen einen Satz repräsentiert. »Satz« wird in der vorliegenden Arbeit Satzdefinition nach Tesnière, definiert als »sprachliche Einheit [...], die sich aus einem Verb (Prädikat) als dem strukturellen Zentrum und einer Reihe von Satzgliedpositionen (Subjekt, Objekte, Adverbialbestimmungen usw.) konstituiert «(Brinker 2005: 23), das heißt, jedes Prädikat bildet einen eigenen Satz. Das Prädikat kann im Ugrischen dabei sowohl verbal als auch nominal sein, ein Kopulaverb ist nicht zwingend notwendig. Aufgrund des pro-drop bestehen viele Sätze lediglich aus einer finiten Verbform. Infinite Verbalformen wurden als subordinierte, eingebettete Nebensätze ebenfalls als Satz interpretiert, jedoch in Relation zum Hauptsatz gezählt. Für das Mansische und größtenteils auch für das Chantische wurden die annotierten Texte von Hand in einzelne Sätze zerlegt und durchnummeriert, für das Ungarische war dies aus Zeitgründen nicht mehr möglich, hier besteht bislang die Zuordnung Satzzeichen - Satz. Sämtliche Sätze haben im Anschluss eine eigene Satz-ID erhalten, welche sich zusammensetzt aus der Abkürzung des Dialektes, einer Angabe zur Textart (ob fortlaufender Text oder Einzelbeleg), einer dreistelligen Nummer im Format 001-999, welche jedem Text zugeordnet wurde, und einer vierstelligen Nummer im Format 0001-9999 für den jeweiligen Satz, zum Beispiel NM_text_001_0001. Eine nachträgliche Aufteilung in mehrere Sätze bzw. Nebensätze ist möglich, da die einzelnen Komponenten gesondert am Ende abgetrennt durch einen Doppelpunkt vermerkt werden, zum Beispiel NM_text_001_0001:3. Da in einem Satz mehrere Possessivsuffixe vorkommen können und damit die Satz-ID nicht zur eindeutigen Zuordnung des Belegs ausreicht, wurden die Tokens im eigentlichen Korpus zusätzlich fortlaufend nummeriert. Ein Verzeichnis der verwendeten Abkürzungen befindet sich am Ende der Arbeit.

\subsubsection{Glossierung, Adaption}

Die Glossierung der Possessivsuffixe erfolgt in der vorliegenden Arbeit nach den Konventionen der Projekte OUL und $\mathrm{OUDB}^{83}$, welche prinzipiell den Leipzig Glossing

83 Ob-UgricDatabase,http://www.oudb.gwi.uni-muenchen.de/index.php?abfrage=corpus_pub_intro\&subnavi= corpus_pub (Zugriff vom 14.05.2017). 
Rules $^{84}$ folgen, mit einigen Anpassungen an die typologischen Eigenschaften der ugrischen Sprachen. Die wichtigste dabei ist die Glossierung der Objektiven Konjugation sowie der Possessivsuffixe: Um die Kodierung zweier Referenten in einem Suffix zu verdeutlichen, werden beide Paradigmen nach dem folgenden Muster glossiert: Numerus des Target bzw. des direkten Objekts > Person und Numerus des Referenzpunktes bzw. des Subjekts.

Des Weiteren ist bei der Glossierung der Possessivsuffixe darauf hinzuweisen, dass keine Segmentierung von Numerusmarker und Personalmarker im Mehrbesitz vorgenommen wird.

Die Numerusmarker wären zwar gut segmentierbar (Chantisch Dual -yət-/-yət-, Plural - $l-$; Mansisch Dual - $\gamma^{-}$, Plural - $n$-, Ungarisch - $i-$ ), dies würde jedoch in der Konsequenz bedeuten, in den Formen des Einbesitzes ein zusätzliches Nullmorphem zu segmentieren, da nicht in allen Konstruktionsarten tatsächlich zwei Referenten kodiert werden und demnach nicht in allen Konstruktionstypen ein zweiter Referent im Singular mit einem Nullmorphem kodiert ist. Auch für den Mehrbesitz in der dritten Person Singular im Ungarischen müsste ein weiteres Nullmorphem eingefügt werden, da dieses Suffix lediglich aus dem Numerus-Zeichen besteht. Die Suffixe werden demnach wie folgt glossiert: das Possessivsuffix der dritten Person Singular, Einbesitz zum Beispiel als »SG<3SG» und Mehrbesitz als »PL<3SG«. Die Reihenfolge der Glossierung entspricht dabei der Reihenfolge der im Possessivsuffix kodierten Referenten: Numerus des Possessum, Person und Numerus des Possessors. Die einzelnen Segmente werden durch das Vergleichszeichen < getrennt. In Konstruktionstypen, in welchen das Possessivsuffix nur einen Referenten kodiert, entfällt dementsprechend der erste Bestandteil vor dem Vergleichszeichen <.

Diese Glossierung der Possessivsuffixe unterscheidet sich damit von der vieler anderer Quellen. Die meisten Handbücher und Chrestomathien verwenden eine Glossierung, welche nur die Kodierung des Referenzpunktes berücksichtigen, zum Beispiel »s1 « für das Possessivsuffix der ersten Person Singular Einbesitz (verglichen Abondolo 1998c), oder »3SG « für das der dritten Person Singular Einbesitz (verglichen Filchenko 2014). In anderen Glossierungen wird die angenommen Possessivität mit vermerkt, zum Beispiel »Px3Du« für das der dritten Person Dual Einbesitz (verglichen Riese 1999). Andere Publikationen arbeiten mit der Verwendung des entsprechenden Personalpronomens der Metasprache, zum Beispiel »the house roof-its (Honti 2006: 47). Auch die Datenbank von Irina Nikolaeva (siehe Anmerkung 75) glossiert lediglich den Referenzpunkt. Theoretisch ist es möglich, diese Datenbank nach einer bestimmten Glosse zu durchsuchen, aufgrund der formgleichen Glossierung erhält man jedoch in den Suchergebnissen nach "3SG« neben dem Possessivsuffix der dritten Person Singular Einbesitz $(\mathrm{SG}<3 \mathrm{SG})$, der dritten Person Singular an Postpositionen und infiniten Verbalformen auch die verbalen Personalmarker der dritten Person Singular in der subjektiven und der objektiven Konjugation. Um dies zu vermeiden, wurde in der Glossierung der Personalsuffixe im OUL-Projekt neben der Mitglossierung eines eventuellen zweiten Referenten für jede Wortart ein eigener Eintrag angelegt.

84 Leipzig Glossing Rules, https://www.eva.mpg.de/lingua/resources/glossing-rules.php (Zugriff vom 14.05.2017). 
So sind die Formen eindeutig zuzuordnen, zum Beispiel »SG<3SG v:Any« für den verbalen Personalmarker, objektive Konjugation vs. »SG<3SG n:Any« für den nominalen Personalmarker dritte Person Singular Einbesitz; »3SG v:Any« für die dritte Person Singular am finiten Verb, »3SG nfin:Any« für die dritte Person Singular am Verbalnomen oder »3SG pstp:Any« für Postpositionen mit Personalmarker dritte Person Singular.

Die ungarischen Texte des Korpus der vorliegenden Arbeit wurden von der Verfasserin selbst glossiert, hier wurden die Konventionen des OUL-Projektes größtenteils übernommen, es wurde jedoch zusätzlich mit einem Null-Morphem gearbeitet. Die deutschen Übersetzungen lagen größtenteils aus verschiedenen Quellen vor und wurden ebenfalls übernommen. Ein vollständiges Verzeichnis der Glossen befindet sich ebenfalls am Ende der Arbeit.

Die Originaltexte aus dem Korpus des OUL-Projektes verfügen über eine englische, russische, deutsche und teilweise ungarische Übersetzung, die Annotation ist mithilfe des FieldWorks Language Explorer (FLEx), einer Open Source Software der SIL Organisation ${ }^{85}$ erfolgt. Die Analysezeilen sind »Word (Wortform), »Morphemes" (Segmentierung in einzelne Morpheme), »Lex. Entries « (Lemma im Wörterbuch ${ }^{86}$ mit Anmerkung zu Dialekt, Verschriftlichung oder anderen Unregelmäßigkeiten falls abweichend von diesem), "Lex. Gloss« (englisches Lemma und Glossen nach Vereinbarungen des OUL Projektes), "Lex. Gram. Info« (Wortart, Informationen zum Wortartwechsel bei Derivation sowie Kombinatorik mit der Wortart bei Morphemen). Das Material wurde für die folgende Arbeit dergestalt adaptiert, dass lediglich die deutschen Übersetzungen - bzw. falls nicht vorhanden, die englischen - und die Analysezeilen »Word «, »Morphemes « und »Lex. Gloss « übernommen wurden, sowie die Zeile »Lex. Gram. Info«, und zwar unbenannt in »PoS« (Part of Speech). Hinzugefügt wurden weitere Zeilen, um die referentielle Bewegung im Text zu annotieren. Diese unterscheiden sich in Art und Anzahl je nach Forschungsstand zum Zeitpunkt der Bearbeitung des jeweiligen Textes. Bei einigen Texten wurde hier außerdem das syntaktische und pragmatische Tagging, welches ebenfalls im Rahmen des OUL-Projektes erfolgt war, mit übernommen. Diese Annotation war erforderlich, da die vorliegende Arbeit im Unterschied zur bisherigen Forschung die Possessivsuffixe nicht isoliert bzw. lediglich als Bestandteil der Possessivkonstruktion betrachtet, sondern eine Analyse innerhalb der Textumgebung anstrebt, um vor allem ihre textstrukturierenden Eigenschaften darstellen zu können. Somit muss man eigentlich von zwei Korpora sprechen.

Dem Korpus der Einzelbelege geht ein annotiertes Textkorpus voraus, um überhaupt Aussagen über die Einzelbelege treffen zu können. Es war demnach nicht nur notwendig, im Vorfeld geeignete Parameter für die Analyse zu erstellen, die Datenverarbeitung konnte darüber hinaus nicht automatisiert erfolgen, da bislang keine ausreichend für die ugrischen Sprachen geeigneten Methoden vorliegen (automatisierte Korpus-Annotationen etc.). Einige Texte aus dem Korpus der vorliegenden Arbeit

85 SIL Fielworks, http://fieldworks.sil.org/flex/ (Zugiff vom 14.05.2017).

86 DEWOS/Steinitz 1966-1993, Баландин/Вахрушева (Hg.) 1958 und Munkácsi/Kálmán (Hg.) 1986. 
wurden mittlerweile durch ein semi-automatisches Annotationstool, welches im Rahmen des OUDB-Projektes entwickelt wurde, syntaktisch, semantisch, pragmatisch und referentiell getaggt. Somit ist inzwischen eine automatisierte Analyse und Auswertung der referentiellen Bewegung im Text möglich, bis zur Einreichung des vorliegenden Promotionsprojektes war jedoch nur eine Analyse von Hand möglich. Die Textanalyse wurde daher aus Zeitgründen auf die unumgänglichen Arbeitsschritte eingeschränkt, von einer detaillierten Analyse sowie Vereinheitlichung wurde im Rahmen der vorliegenden Arbeit daher abgesehen, da lediglich die referentielle Bewegung im Zusammenhang mit der Verwendung der Possessivsuffixe berücksichtigt wurde und nicht die Darstellung des gesamten reference-tracking beabsichtigt war.

\subsubsection{Auswahl und Systematisierung der Daten}

Das eigentliche Korpus der vorliegenden Arbeit, die analysierten Vorkommen der Possessivsuffixe, besteht insgesamt aus 2674 Token, welche sich in etwa gleichmäßig auf die drei ugrischen Sprachen Chantisch, Mansisch und Ungarisch verteilen. ${ }^{87}$ Die chantischen Belege repräsentieren dabei die nördliche sowie die östliche Dialektgruppe, mit Daten aus dem Kazym- bzw. dem Surgut-Chantischen. Die mansischen Belege repräsentieren den Nord-Dialekt, wobei eine weitere dialektale Zuordnung nicht möglich ist, in der Korpus-Übersicht ist jedoch der Ort der Sammlung, soweit rekonstruierbar, mit angegeben. Im Ungarischen wurde keine dialektale Unterteilung vorgenommen. Die Auswahl der Dialekte ist der Zugänglichkeit des Materials geschuldet, da es sich beim Nord-Mansischen um die Dialektgruppe mit der größten Sprecherzahl handelt, und mit den Kazym- und Surgut-Dialekten des Chantischen eine kontrastive Analyse zweier Dialektgruppen ermöglicht wurde, ist die Auswahl für aussagekräftige Analysen dennoch gut geeignet (siehe auch Kapitel 1.1).

Nicht alle Vorkommen von Possessivsuffixen wurden jedoch in das Korpus aufgenommen. Zum einen gibt es Belege, welche nicht eindeutig bestimmt werden können. Dazu gehören zum Beispiel Segmentierungen von möglicherweise schon fossilisierten Formen, zum Beispiel chantisch xattatzn, am Tage. Diese Form ist einerseits als Einheit im DEWOS vermerkt (DEWOS/Steinitz 1966-1993: 572), es ist außerdem nicht möglich, aufgrund des begrenzten vorliegenden Materials mit anderen Belegen zu vergleichen, ob auch andere Kombinationen zum Beispiel mit einer anderen Person oder einem anderen Kasussuffix vorkommen können oder ob es sich bei der betreffenden Form um eine Grammatikalisierung handelt. Von einer Segmentierung wurde deshalb abgesehen. Zu einem späteren Zeitpunkt wäre denkbar, sich explizit mit solchen Formen zu befassen und diese mithilfe gezielter Elizitierungen einzuordnen. Nicht aufgenommen in das Korpus wurden auch Belege, in welchen das Possessivsuffix nicht eindeutig abgetrennt werden kann, da es sich entweder um fossilisierte Formen wie Personalpronomina handelt oder um sehr archaische Formen, die ohne Possessivsuffix nicht mehr als eigene Lemmata existieren (zum Beispiel die 
archaischen Anredepronomina kelmed/kelmetek/kigyelmetek des Ungarischen). Bei Personalpronomina erscheint im Ob-Ugrischen oft als Stammerweiterung das jeweilige Possessivsuffix, zum Beispiel mansisch änəmn ,1SG.DAT'; oder sie sind an der Bildung der Reflexivpronomina beteiligt (Riese 2001: 31). Auch das ungarische Reflexivpronomen maga (verglichen magam ,1SG', magunk,1PL') enthält ein mehr oder weniger fossilisiertes Possessivsuffix, weswegen die Form ebenfalls nicht in das Korpus aufgenommen wurde. ${ }^{88}$ Des Weiteren wurde im Zweifelsfall gegen eine Aufnahme in das Korpus entschieden, wenn aufgrund der Qualität der Quelle eine Form nicht eindeutig erkennbar war. Dies gilt insbesondere für die handschriftlichen Aufzeichnungen von Valerij Nikolajevič Černecov, die nur in einer grobkörnigen Kopie vorliegen.

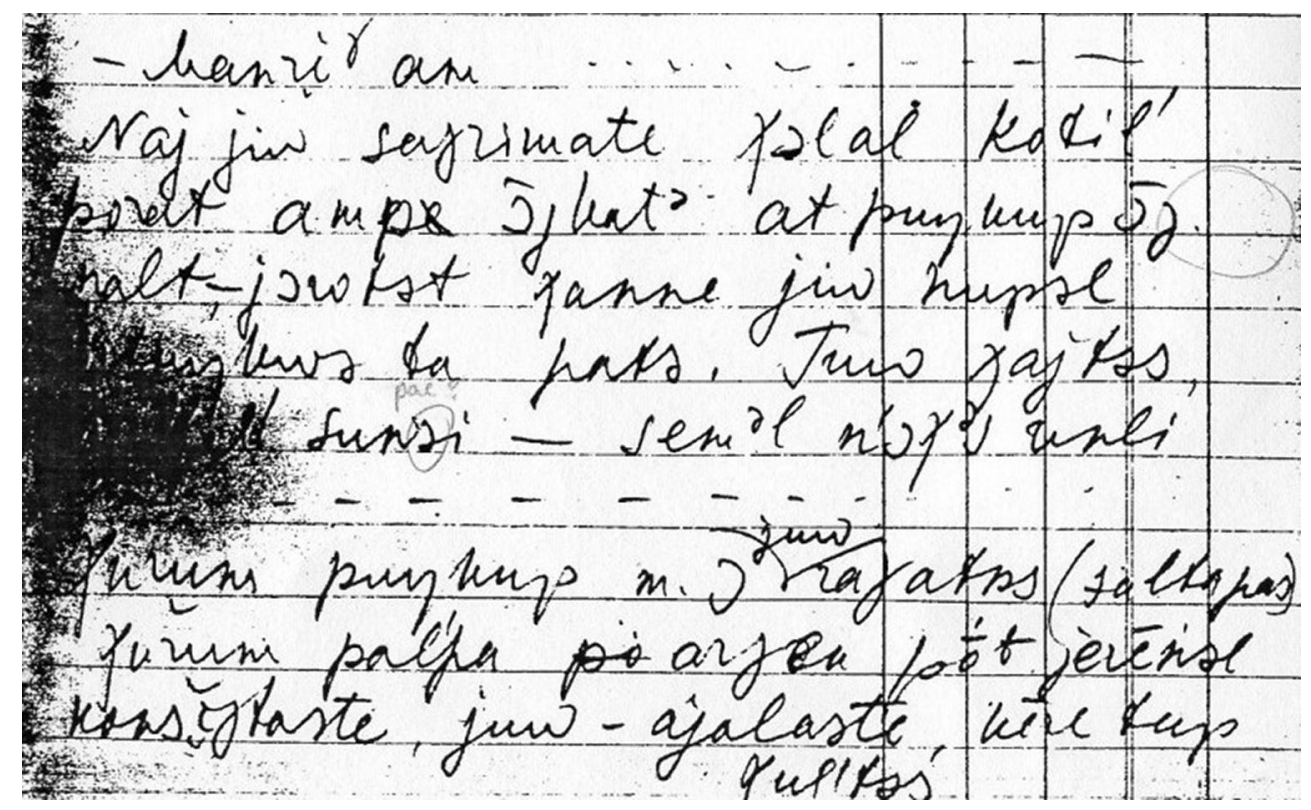

Abbildung 8: Schriftprobe aus den Aufzeichnungen von Valerij Nikolajevič Černecov. (C) 2012 OUL, http:// www.oudb.gwi.uni-muenchen.de/index.php?abfrage=fieldwork_chernetsov (Zugriff vom 22.05.2017).

Ebenso wurde mit festen Phrasen und Redewendungen verfahren, das heißt, Konstruktionen, in welchen die Ergänzung immer ein Possessivsuffix trägt und welche auch so in den verwendeten Wörterbüchern zu finden ist. Für das Ungarische sind dies beispielsweise:

88 Reichert 1986 trennt die Personalmarker zwar von allen Pronomina ab (Reichert 1986: 62f.), da aber das Reflexivpronomen maga im Wörterbuch (Hessky (Hg.) 2002: 772) und im HNC (HNC_Abfrage_0038) zumindest in der dritten Person als eigener Lemma-Eintrag geführt wird, werden diese Formen in der vorliegenden Arbeit nicht segmentiert. 
annak érdekében, hogy Tendenz zu fester Phrase, verglichen HNC_Abfrage_0025.pdf und HNC_ Abfrage_0035.pdf, HNC_Abfrage_0036.pdf: Zwar macht die Konstruktion annak érdekében nur etwas mehr als ein Zehntel aller Vorkommen von érdekében aus, die Anzahl an Vorkommen von érdekében, hogy entspricht dem jedoch ebenfalls in etwa. Eine gezielte Suche nach allen drei Komponenten ist aufgrund der Suchmaske des HNC nicht möglich (siehe Beschreibung HNC).

Aus diesem Grund wurde erdek(ében) als Beleg aufgenommen, annak érdekében hogy jedoch nicht.

bü-ébá-é

Phrase, ,weder gicks noch gacks' (Halász/Földes/Uzonyi (Hg.) (2006): 179), nicht aufgenommen;

éjszaka

Substantiv, ,Nacht' (Hessky (Hg.) 2002: 251), nicht aufgenommen;

erönek erejével

Phrase, ,mit aller Gewalt' (Halász/Földes/Uzonyi (Hg.) (2006): 384), nicht aufgenommen;

eszébe jutni

Phrase, verglichen vkinek eszé bejutni vmi ,jemandem fällt etwas ein' (Halász/ Földes/Uzonyi (Hg.) (2006): 398), nicht aufgenommen;

felebotját hajtani

Phrase, verglichen fülebotját sem mozgatja, er rührt weder Hand noch Faust' (Halász/Földes/Uzonyi (Hg.) (2006): 168), nicht aufgenommen;

hányadán

Adverb, ,wie' (Halász/Földes/Uzonyi (Hg.) (2006): 602), nicht aufgenommen;

jó tét helyébe

kutyafuttában

Redewendung, verglichen jótett helyébe jót ne várj, erwarte nicht Gutes für eine gute Tat' (MK 27), nicht aufgenommen;

Adverb, ,in aller Eile' (Halász/Földes/Uzonyi (Hg.) (2006): 894), nicht aufgenommen;

lohalálába/n

Adverb, ,Hals über Kopf' (Halász/Földes/Uzonyi (Hg.) (2006): 957), nicht aufgenommen;

mindnyájában

Adverb, ,allesamt', (verglichen Halász/Földes/Uzonyi (Hg.) (2006): 1039), nicht aufgenommen

szerelem záloga

Phrase, ,Das Pfand der Liebe' (Halász/Földes/Uzonyi (Hg.) (2006): 1621), nicht aufgenommen

szerét

Phrase, verglichen szerét ejti ,eine Gelegenheit finden' (Hessky (Hg.) 2002: 1058), nicht aufgenommen;

torkaszakadtából Phrase, verglichen torkaszakadtából kiabál, sich die Kehle aus dem Halse schreien' (Hessky (Hg.) 2002: 1117), nicht aufgenommen;

tövéröl hegyére

Phrase, verglichen töviröl hegyire ,haarklein' (Halász/Földes/Uzonyi (Hg.) (2006): 1465), nicht aufgenommen;

vége hossza sincs

Phrase, verglichen se vége, se hossza ,es nimmt kein Ende' (Hessky (Hg.) 2002: 651), nicht aufgenommen;

végtére

Adverb, ,schließlich' (Halász/Földes/Uzonyi (Hg.) (2006): 1565), nicht aufgenommen;

vkinek gondját viselni Phrase, für jemanden Sorge tragen' (Halász/Földes/Uzonyi (Hg.) (2006): 550), nicht aufgenommen;

vkivel van dolga

Phrase, , mit jemandem zu tun haben' (Hessky (Hg.) 2002: 222), nicht aufgenommen;

Interjektionen wie (hát) uram fia! in etwa ,Herrje!' oder jaj lelkem teremtette! in etwa ,Menschenskinder!' wurden ebenfalls nicht berücksichtigt. Possessivkonstruktionen mit dem Possessivmarker -é (dem sogenannten anaphorischen Possessivpronomen, 
Kornai 1985: 84) sind ebenfalls nicht Bestandteil der Analyse, da es sich hier um eine andere Art von Suffix handelt, welches nicht zum Paradigma der Possessivsuffixe zählt.

Für die ob-ugrischen Sprachen ist eine so detaillierte Aussage aufgrund der Dokumentationslage nicht möglich, das heißt, es können keine Aussagen über feste Konstruktionen, Phrasen oder archaische Wendungen und auch zu fossilisierten Formen nur in einem geringen Umfang gemacht werden. Nach Abzug der hier skizzierten Fälle, in welchen das Possessivsuffix nicht ohne Weiteres segmentierbar oder konstruktionsbedingt obligatorisch ist, wurden die restlichen Vorkommen in das Korpus aufgenommen. Im Verlauf der Analyse wurden dann weitere Belege ausgeschlossen, etwa da sich durch die Auswertung bestimmter Parameter Gruppierungen von Konstruktionen ergeben haben, die ebenfalls Tendenzen von Grammatikalisierungen zu festen Phrasen oder obligatorischen Verwendungen des Possessivsuffixes aufweisen. Diese werden im Verlauf der Arbeit thematisiert.

Ein weiteres Problem hat sich aus den Komposita ergeben: Hier musste unterschieden werden zwischen rechten Komposita (in welchen sich die Bedeutung gegenüber den Einzelteilen verändert), welche auch im Wörterbuch als zusammengesetzte Form verzeichnet sind, und solchen Formen von Wortzusammenschreibungen, welche nicht explizit im Wörterbuch aufgeführt sind, wie das Beispiel aus Honti 2007a, ház tetö ,Hausdach': Es wird im Wörterbuch nicht als Kompositum geführt, im HNC jedoch mit 111 Belegen zusammen geschriebenen gegenüber zwei getrennt geschriebenen Belegen (HNC_Abfrage_0039 und HNC_Abfrage_0040). Dieser Beleg ist exemplarisch für die zahlreichen Entscheidungen, welche bei der Behandlung der Belege erfolgen mussten. Wenn nicht einmal für das Ungarische hier Richtwerte uneingeschränkt übernommen werden können, so ist es im Ob-Ugrischen erst recht nicht möglich.

Die ob-ugrischen Sprachen besitzen darüber hinaus keine ausreichend manifestierten Rechtschreibregeln, als dass die Orthografie eines Wortes generell als Kriterium herangezogen werden könnte; und auch bei Zusammenschreibungen muss daher mit Bedacht vorgegangen werden, zumal eine Wortkombination durchaus einmal als Zusammensetzung und einmal getrennt vorkommen kann:

(23) NM_text_004_0093

$\begin{array}{llllllll}\text { kolkan } & -n & \text { ti } & \text { pora } & -t & \text { ti } & \text { porizm } & \text {-as } \\ \text { floor } & \text {-DLAT } & \text { EMPH2 } & \text { time } & \text {-LOC } & \text { EMPH2 } & \text { jump } & \text {-PST[3SG] }\end{array}$

'Dann sprang er auf den Fußboden.'

(24) NM_text_003_0065

\begin{tabular}{|c|c|c|c|}
\hline $\begin{array}{l}e k^{w} a \\
\text { old woman }\end{array}$ & $\begin{array}{l}\text { kol kan } \\
\text { floor }\end{array}$ & $\begin{array}{l}-t \\
-\mathrm{LOCC}\end{array}$ & $\begin{array}{l}\text { un } \\
\text { sit }\end{array}$ \\
\hline
\end{tabular}

'Die Tante saß auf dem Fußboden.'

Die Auswirkungen auf die Analyse und ihre Handhabung sind sowohl bei den einzelnen Konstruktionen (Kapitel 5.1) als auch bei den Mechanismen (Kapitel 5.2) beschrieben. Komposita, welche an beiden Bestandteilen ein Possessivsuffix tragen, wurden als jeweils ein eigener Beleg im Korpus vermerkt, da sich bei den für die Analyse relevanten Vorkommen die Bedeutung des Kompositums gegenüber den Einzelteilen nicht verändert. 


\subsubsection{Analyse der einzelnen Belege}

Es wurde versucht, die einzelnen Belege im Korpus nach bestimmten Kriterien zu analysieren, welche Gemeinsamkeiten, aber auch Unterschiede aufzeigen sollen, um die Verwendungen der Possessivkonstruktionen systematisieren zu können. Die Analyse der Possessivsuffixe wurde wie folgt durchgeführt: Als Metadaten wurden zu jedem Beleg die fortlaufende Nummerierung sowie die Satz-ID (siehe Beschreibung Annotation) vermerkt, des Weiteren die gesamte Konstruktion, wie sie im Text vorkommt (»Form«), gesondert noch einmal das jeweilige Suffix (in all seinen vorkommenden Allomorphen), die Übersetzung des Targets (»Lemma») und die Glossierung desselben. Am Ende der Tabelle befinden sich außerdem der gesamte Satz, die Glossierung und die Übersetzung (deutsch, wenn vorhanden, sonst englisch). Die Spalte »Dialekt» wurde zur Sortierung benötigt.

Dabei hat sich herausgestellt, dass diese Analysekriterien eine erste Strukturierung erlauben, aber nicht ausreichen bzw. bestimmte Eigenschaften nicht fassen können, weswegen eine Analyse der einzelnen Bestandteile der Konstruktionen folgte, um Hinweise zum Beispiel auf die Aktiviertheit eines Referenten zu erhalten. Hierbei handelt es sich um jeweils eine Spalte für die Realisierung des Referenzpunktes (»ReFPunKT» als Leerstelle, Pronomen oder Substantiv), für die Wortart des Kopfes (zum Beispiel Subs-Px oder VN-Px) sowie eine Spalte zur Notation eines möglichen Determinierers. Bei der Bestimmung der Wortart musste zusätzlich die Problematik Postposition gegenüber Substantiv berücksichtigt werden. Einige Lemma kommen im Wörterbuch sowohl als Postposition als auch als Substantiv vor.

$[\rightarrow$ superentry] $\cdot \mathrm{N}$ jat $\sim$ jot, LM jät $\sim$ jot, LU jät $(\sim$ ät $), \mathrm{P}$ jät, $\mathrm{K}$ jät $\sim$ jøt $(\sim$ jŏt), $\mathrm{T}$ jët $\sim$ jät $\mathrm{I}$. közép 2. középsô |1. Mitte 2. mittlere

Abbildung 9: Überschneidung der Wortart Substantiv und Postposition in Munkácsi/Kálmán 1986. C 2012 OUL, http://www.oudb.gwi.uni-muenchen.de/index.php?abfrage=munka\&subnavi=edictionary (Zugriff vom 22.05.2017).

Da dies sowohl Auswirkungen auf die Zuordnung zum Konstruktionstyp als auch auf die Anzahl der kodierten Referenten hat, musste hier ebenfalls eine einheitliche Vorgehensweise festgelegt werden. War keine eindeutige Entscheidung zu treffen, wurde zumindest die Wortart für weitere Vorkommen desselben Lemmas einheitlich bestimmt. Im Verlauf der Analyse hat sich dabei eine Reihe von Gemeinsamkeiten herausgestellt, welche eine Gruppierung einiger dieser Doppelformen zulässt, auch wenn die Wortart nicht abschließend bestimmt werden kann. Dies wird unter Punkt "Kollokation« in Kapitel 5.2.2.2 nochmals thematisiert. Es ist ebenfalls möglich, dass in einigen Konstruktionen das Possessivsuffix an den Bestandteil der Konstruktion rückt, welches den rechten äußeren Rand bildet, auch wenn dies nicht der Kopf der Konstruktion ist. Dies ist beispielsweise bei Adverbialbestimmungen mit Postpositionen möglich oder bei Partikeln. Hier wurde die Konstruktion der Wortart zugeordnet, die den tatsächlichen Kopf der Konstruktion bildet. 
Die Belege des Ungarischen des Konstruktionstyps Subs PostP-Px repräsentieren einzig und allein die Konstruktion mit der Postposition számára ,für ihn/sie؛. Sie gehört zu der Gruppe von Belegen, bei welchen von vornherein infrage gestellt war, ob sie im Korpus der vorliegenden Arbeit aufgenommen werden oder nicht. Die Worform számwurde zunächst mit zu den Postpositionen gezählt, da sie a) dem Bezugswort folgt und b) ein Possessivsuffix enthält. Die Segmentierung war jedoch schon aufgrund des folgenden, produktiven Kasussuffixes fraglich (zur Problematik der Segmentierung siehe auch Ausschluss von Belegen). Sowohl im Korpus als auch HNC finden sich ebenfalls Belege mit anderen Formen des Possessivsuffixes, zum Beispiel számodra.

Im HNC sind diese jedoch uneinheitlich glossiert, mal als eine Einheit als Adverb, mal segmentiert als Substantiv mit Possessivsuffix und Kasussuffix (dabei könnte es sich um eine Interferenz zum Substantiv szám, Zahl' handeln, da im HNC keine Möglichkeit besteht, die Bedeutung mit in die Suchanfrage aufzunehmen). Bei Hessky (Hg.) 2002 wird lediglich die Form számára verzeichnet, und zwar als Adverb mit der Bedeutung ,für‘ ohne Unterscheidung nach Person (Hessky (Hg.) 2002: 1038). Reichert 1986 behandelt die Form számára unter den Postpositionen und segmentiert sowohl Possessivsuffix als auch Kasussuffix. Allerdings glossiert er den Stamm als das Substantiv ,Zahl' (Reichert 1986: 73f.). Ein gemeinsamer Ursprung bzw. eine Grammatikalisierung der Form számára, für' aus dem Substantiv ,Zahl' mag anzunehmen sein, allerdings ist allein durch den Bedeutungswandel eindeutig von einer eigenen Wortform auszugehen, welche nicht unbedingt segmentiert werden kann. Belege mit számára und számodra wurden daher nicht in das Korpus der vorliegenden Arbeit aufgenommen, und die Wortart wird als Adverb klassifiziert. Konstruktionen mit dem Lemma szám,(An)Zahl wurden als Belege der Konstruktion Subs-Px gewertet.

In der Spalte für den Determinierer werden sowohl Vorkommen von Demonstrativpronomen wie im Beispielsatz (21) als auch des definiten Artikels im Ungarischen vermerkt (Beispiel 20), des Weiteren bestimmte Pro-Formen (siehe dazu Kapitel 5.1.1). Die Reihenfolge (Determinierer - Refpunkt - Kopf) spiegelt dabei nicht notwendigerweise die korrekte Reihenfolge in der Konstruktion wider. Begründet ist dies in dem Versuch, die verschiedenen Realisierungen der Konstruktion in eine einfache schematische Darstellung zu übertragen. Weitere Bestandteile, zum Beispiel adjektivische Attribute wurden nicht vermerkt (zur Bestimmung der Wortarten in der Konstruktion siehe Kapitel 5.1.1). Die folgende Spalte enthält eine Bestimmung der in der Konstruktion ausgedrückten Relation zwischen Referenzpunkt und Kopf (siehe dazu Kapitel 5.1.1.4), welche jedoch nur bei der Kodierung von zwei Referenten existiert. Wird nur ein Referent kodiert, bleibt die Spalte der Relation leer bzw. ist grau hinterlegt.

Ausgehend von der Analyse dieser Parameter werden in Kapitel 5.1 die einzelnen Konstruktionen und deren Vorkommen im Korpus thematisiert. Da es im Kapitel 5.2 auch um die Textumgebung von Konstruktionen mit Possessivsuffixen und Textstrukturierung durch Konstruktionen mit Possessivsuffixen geht, genügte es jedoch nicht, den Beleg allein zu analysieren, sondern auch dessen Umgebung (Kontext), weswegen weitere Parameter, welche die Vorerwähnung von Referenzpunkt und Target betreffen, hinzugezogen wurden. Als Vorlage diente hierzu die Arbeit von Kern 2010, welche sich mit einer ähnlichen Fragestellung im Französischen befasst. Bei beiden wurde der sogenannte »look-back« vermerkt, die Kodierung der letzten Vorerwähnung. Diese 
Parameter wurden anhand von Givón 1983 erstellt, und mit eigenen Bezeichnungen kategorisiert ${ }^{89}$ : »neu « - »im selben Satz« - »im Satz zuvor« - »im Text«. Dazu kommen Parameter, die die Vorerwähnung des Referenzpunktes mit der Realisierung in der Konstruktion verknüpfen (»neu, in der Konstruktion« - »in der Konstruktion«). Eine gemeinsame Vorerwähnung von Referenzpunkt und Target wird ebenfalls notiert (»Dual im Satz zuvor « - »Dual im Text zuvor« - »in der Gruppe im Satz zuvor «) oder auch der Sprechaktteilnehmer als Referenzpunkt. Da auch Referenzpunkt und Target die Rollen wechseln können, wurde dies ebenfalls vermerkt (zum Beispiel »REFPUnKT/Target im Satz zuvor« - »REFPunKT/Target im selben Satz«). Es wurde außerdem vermerkt, ob das Target durch den Handlungsverlauf indirekt vorerwähnt ist, zum Beispiel als Resultat einer Verbalhandlung. Wird nur ein Referent kodiert, bleiben die entsprechenden Spalten für das Target in diesen Fällen leer, bei den infiniten Verbalkonstruktionen wurde jedoch vermerkt, ob der kodierte Referent im Hauptsatz fortgeführt wurde oder nicht. Im Verlauf der Analyse haben sich einige dieser Parameter bewährt, andere jedoch haben sich als weniger hilfreich erwiesen (zum Beispiel der Wert »in der Konstruktion « - überdies durch die Analyseparameter II ableitbar - hat die hilfreichere Angabe »im Satz zuvor « verzerrt und musste teilweise nachbearbeitet werden).

Der Notation von Referenzpunkt und Target sowie deren Angaben zum »lookback « ging eine entsprechende Analyse in den jeweiligen Texten voraus. Hierfür wurden die einzelnen in den Handlungen vorkommenden Referenten markiert sowie die referentiellen Verweismittel ihnen jeweils zugeordnet. Die Häufigkeit der Kodierungen der jeweiligen Referenten bildete zudem die Grundlage zur Zuordnung der einzelnen topikalen Rollen. So wurden zum Beispiel für den Text NM_text_004 nach der zuvor beschriebenen Zählweise 197 Sätze errechnet. Auf den Referenten, welcher als DiskursTopik bestimmt wurde, ist 146-mal im Textverlauf verwiesen worden, mitunter in topikalen Ketten mit bis zu 15 Sätzen am Stück.

Das vorwiegende referentielle Verweismittel ist die Nullanapher und auch bei der Re-Aktivierung sind Verweismittel, welche eine hohe Topikalität voraussetzen, zum Einsatz gekommen. Auf Referenten, welche als Paragraph-Topik bestimmt wurden, wurde zwischen 30- und 40-mal im Text verwiesen. Die topikalen Ketten sind entsprechend kürzer. Erwähnenswert ist außerdem das Vorkommen bestimmter Referenten in mehreren Paragraphen des Textes. Hierzu wurde zunächst anhand des Handlungsverlaufs der Text in Paragraphen (darunter frame-setting und Ende) und eventuell mehrere Subsets eingeteilt. Das Diskurs-Topik tritt in der Regel in allen Paragraphen und auch in den meisten Subsets auf, und auch Paragraphen-Topiks sind in mehreren Paragraphen an der Handlung beteiligt. Satz-Topiks hingegen kommen nur innerhalb eines Paragraphen oder eines Subsets vor. Deshalb wurden Referenten, welche an sich seltener erwähnt wurden, dies jedoch über mehrere Paragraphen der Fall war, als Paragraph-Topik bestimmt, und Referenten, welche zwar relativ häufig erwähnt wurden, dies jedoch innerhalb eines kurzen Abschnitts des Handlungsverlaufs, als Satz-Topiks bestimmt. Eine solche Bestimmung ist manuell erfolgt und konnte daher nicht für alle

89 Givón 1983: 13f. gibt die Anzahl der Sätze zwischen dem aktuellen Vorkommen und der letzten Vorerwähnung als einen numerischen Wert an, die vorliegende Arbeit verwendet eine vereinfachte, den Analysekriterien der vorliegenden Arbeit angepasste Variante. 
Texte durchgeführt werden. Letztlich wurden die daraus gewonnenen Einsichten unterstützend herangezogen, sind aber nicht im eigentlichen Korpus vermerkt. Hier sei noch einmal das semi-automatische Annotationstool des OUDB-Projektes erwähnt, welches durch das Tagging der einzelnen Referenten deren Vorkommen im Text genauer bestimmen kann. Die Aufnahme dieser zusätzlichen Analyseparameter zu einem späteren Zeitpunkt könnte weitere lohnende Erkenntnisse liefern.

Um die Funktionen der Konstruktionen mit vorerwähntem Target weiterhin unterteilen zu können, wurde in diesen Fällen (das heißt, Wert der Spalte »look-back» Target ist nicht »neu«) zusätzlich eine syntaktisch-semantische Analyse von Referenzpunkt und Target im unmittelbar vorangehenden, im aktuellen und um Folgesatz vorgenommen (»Syn/Sem ReFPunkt Satz vorher« - »Syn/Sem ReFPunkT» - »Syn/Sem ReFPunk T Folgesatz« - »Syn/Sem Target Satz vorher « - Syn/Sem Target « - »Syn/ Sem Target Folgesatz $«$ :

Hierbei wurden die Bezeichnungen auf ein kleines Set beschränkt, das heißt, es wurde beispielsweise nicht zwischen Experiencer, Force und Agens oder Theme und Patiens oder Recipient und Goal unterschieden (diese wurden jeweils als Agens respektive Patiens oder Goal getaggt). Des Weiteren beschränken sich die semantischen Rollen der adverbialen Ergänzungen auf Source - Location (darunter Time) - Goal und Instrumental. Lediglich bei den belebten Referenten wurde noch nach Adressee und Comitative unterschieden.

Diese Parameter bilden zusammen mit den Beschreibungen aus Kapitel 5.1 die Grundlage zur Kategorisierung nach textstrukturierenden Eigenschaften der einzelnen Konstruktionen in Kapitel 5.2. Kapitel 5 besteht demnach aus zwei Analyseansätzen: (a) eine Beschreibung der einzelnen Vorkommen systematisiert nach der jeweiligen Konstruktionsart und (b) eine Systematisierung anhand von Vorerwähntheit und Realisierung von Referenzpunkt und Target in Relation zum Textverlauf. Die Ergebnisse der Analyse sind in den Spalten »Mechanismus» (siehe Kapitel 5.2) und »Funktion» (siehe Kapitel 6) vermerkt.

Weitere Analysekriterien wie die Position der NP im Satz wurden ebenfalls angedacht, um die Eigenschaften der jeweiligen Vorkommen auf allen linguistischen Analyse-Ebenen beschreiben zu können. Da jedoch die Übersichtlichkeit und Einheitlichkeit bei der manuellen Analyse der Daten nur bis zu einem gewissen Umfang gewährleistet ist, wurde die Analyse so gründlich wie möglich und gleichzeitig so kompakt wie möglich gehalten und ist in der vorliegenden Form an der Grenze des Möglichen ohne automatisierte Unterstützung. Zu einem späteren Zeitpunkt können und sollen die Parameter jedoch anhand neuer Kenntnisse, der Hinzunahme weiterer Dialekte und mithilfe automatisierter Abfragefunktionen modifiziert werden. Für die vorliegende Arbeit wurde lediglich eine erste Kategorisierung und Analyse der einzelnen Konstruktionen angestrebt. 



\section{Ergebnis und Diskussion}

\subsection{Die einzelnen Konstruktionen}

Das folgende Kapitel enthält die Ergebnisse der in Kapitel 4.2 beschriebenen Datenanalyse. Die Analyse der Korpus-Belege widmet sich zunächst der Konstruktionsart ausgehend vom Kopf der Konstruktion. Hier werden die folgenden drei Konstruktionstypen unterschieden: Konstruktionen mit Substantiv, Verbalnomina und Postpositionen. Zur Veranschaulichung sei hier noch einmal die Tabelle aus Kapitel 1.2 dargestellt:

\begin{tabular}{lllll}
\hline Typ & Modifizierer & Kopf & & Abkürzung \\
\hline 1 & Relativsatz/Substantiv/Pronomen/ $\varnothing$ & Substantiv & $-\mathrm{Px}$ & SUBs-Px \\
\hline 2 & Substantiv/Pronomen/ $\varnothing$ & Verbalnomen & $-\mathrm{Px}$ & VN-Px \\
\hline 3 & Substantiv/Pronomen & Postposition & $-\mathrm{Px}$ & PosTP-Px \\
\hline
\end{tabular}

Tabelle 14: Verteilung der Korpusbelege auf die einzelnen Konstruktionen mit Possessivsuffix.

\subsubsection{Die Konstruktion Substantiv-Possessivsuffix (SuBs-Px)}

Die Konstruktion Subs-Px wird in der vorliegenden Arbeit fokussiert. Sie ist die am häufigsten vorkommende Konstruktion mit Possessivsuffixen. Es ist die Konstruktion, welche vornehmlich in der Forschung beschrieben wird, wenn es um den Ausdruck von Possession in den ugrischen Sprachen geht, und von welcher sich daher der Begriff "Possessivkonstruktion « und somit »Possessivsuffix" ableitet. Sämtliche anderen Arten der Possessivkonstruktion - prädikativ oder mit externem Possessor - werden als Grammatikalisierungen mit Ursprung in dieser Konstruktion angesehen (verglichen Kenesei/Vago/Fenyvesi (Hg.) 1998: 157). Auch in einem semasiologisch ausgerichteten Forschungsansatz ist der Konstruktionstyp SuBs-Px der am häufigsten vorkommende, wenn auch nicht unbedingt sämtliche Vorkommen als Ausdruck von Possession angesehen werden können. Im folgenden Kapitel wird diese Konstruktion näher beschrieben, das heißt, ihre Bestandteile werden vorgestellt sowie thematisiert, und ihr Vorkommen wird im Korpus beschrieben.

\subsubsection{Attribute der Konstruktion SuBs-Px}

Wie in Kapitel 1.2 bereits erwähnt, ist die Possessivkonstruktion eine nominale Attributivkonstruktion, das heißt der Kopf kann mit Attributen erweitert werden, welche dem Kopf vorangestellt werden. Als Attribute kommen Substantive, Adjektive, Pronomina, Numeralia sowie Determinantien infrage. Bei den Konstruktionen Subs-Px ist dabei die Frage von hoher Relevanz, welches der Attribute im Possessivsuffix kodiert ist, oder umgekehrt, in welcher Form der im Possessivsuffix kodierte Referent als 
Attribut erscheinen kann. Dies ist nicht immer auf den ersten Blick zu entscheiden, da beispielsweise Adjektive in den ugrischen Sprachen formal nicht von vornherein von Substantiven abgrenzbar sind (zum Beispiel Nikolaeva 1999: 20; Filchenko 2007: 130); doch für die vorliegende Arbeit ist entscheidend, ob der Kopf der Nominalphrase von einem Adjektiv charakterisiert wird oder mit einem zweiten Substantiv in Relation gestellt wird (Referenzpunkt). Zwar gibt es in allen ugrischen Sprachen bestimmte denominale Ableitungssuffixe - zum Beispiel mansisch - tal, $-\eta$, chantisch - $l i,-\eta$, ungarisch - $i$ $-s$, welche adjektivische Verwendung ausdrücken; diese sind jedoch nicht obligatorisch, weswegen eine Abfolge von zwei Nomina als Adj Subs oder auch Subs Subs gelesen werden kann, zum Beispiel:

(25) NM 1805

$\begin{array}{lll}\text { kol } & \text { ala } & - \text { te } \\ \text { house } & \text { roof } & -\mathrm{SG}<3 \mathrm{SG}\end{array}$

'Das Dach des Hauses.'

(26) NM 2202

$\begin{array}{llll}\text { aryon } & \text { po:t } & \text {-anel } & -n \\ \text { copper } & \text { kettle } & -\mathrm{SG}<3 \mathrm{PL} & -\mathrm{DLAT}\end{array}$

'Their kettle made from copper.'

Ein Adjektiv beschreibt häufig bestimmte Eigenschaften eines Referenten, sodass die Klassifizierung semantisch basiert ist, zum Beispiel im Ost-Chantischen nach Kriterien wie Dimension, physikalischer Form, Geschmack, Gefühl oder Wert (Filchenko 2007: 130f.). Die syntaktisch-basierte Definition des Adjektivs als Wortart, die »beim Nomen stehend attributive Verwendung« (Bußmann 2002: 47f.) ausübt, oder die Eigenschaft, von einer Kopula regiert werden zu können, die zur Bestimmung von Adjektiven eingesetzt werden kann (Bußmann 2002: 47f.), sind in kopulalosen Sprachen ebenfalls nicht ohne Weiteres zur Bestimmung heranziehbar. Lediglich die Möglichkeit der Komparation, verbunden mit der bestimmten formalen Kennzeichnung, ist ausschließlich Adjektiven zu eigen, kann jedoch auch keine eindeutige Bestimmung für die Positivform liefern.

Die Definition von Adjektiven im den ugrischen Sprachen erfährt demnach dieselben Schwierigkeiten wie die der Substantive (siehe Kapitel 1.2). Hilfreich ist es, die relative Position der jeweiligen Attribute in der Konstruktion zu ermitteln: Adjektivisch gebrauchte Attribute behalten ihre relative Stellung zum Kopf auch dann bei, wenn die Satzstellung verändert ist (Murphy 1968: 135), wohingegen zwischen substantivischem Attribut (dem Referenzpunkt) und Kopf weitere Attribute eingefügt werden können, da die Zugehörigkeit zum Kopf durch das Possessivsuffix markiert ist.

(27) SK_text_007_0062:1

\begin{tabular}{|c|c|c|c|c|c|c|c|}
\hline $\begin{array}{l}\text { b:t } t^{j} e \\
\text { father }\end{array}$ & $\begin{array}{l}-m \\
-S G<1 S G\end{array}$ & $\begin{array}{l}\text { i:t } \\
\text { now }\end{array}$ & $\begin{array}{l}\text { sem } \\
\text { eye }\end{array}$ & $\begin{array}{l}-\gamma \partial 4 \\
-D U<3 S G\end{array}$ & $\begin{array}{l}\text { D:təm } \\
\text { bad }\end{array}$ & $\begin{array}{l}-\gamma \partial \\
- \text { TRNS }\end{array}$ & $\begin{array}{l}\text { jək } \\
\text { become[PST] }\end{array}$ \\
\hline
\end{tabular}

'Now that my father's eyes cannot see well.'

Allen Arten von nominalen Attributen gemeinsam ist, dass sie etwas zur Identifizierung des Kopfes beitragen, indem durch die Information, welche die Attribute ausdrücken, etwas zur Beschreibung hinzugefügt wird, wodurch der betreffende Referent gegenüber anderen möglichen Referenten eingegrenzt wird. Im Vordergrund für die vorliegende Arbeit steht dabei, dass nur bestimmte nominale Attribute koreferent 
mit dem im Possessivsuffix kodierten Referenten sind, während die anderen den Kopf evaluieren. Des Weiteren kann der Referent, welcher als Kopf fungiert, nur mit einem anderen Referenten (in Form eines Substantives) in Bezug gesetzt werden, wohingegen mehrere evaluierende Attribute in der Nominalphrase vorkommen können. ${ }^{90}$

(28) NM_text_007_010

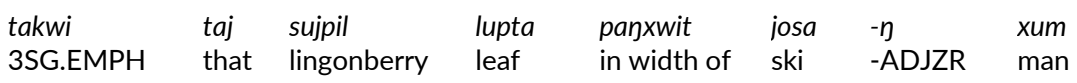

'Er war ein Mann, mit Skiern schmal wie Preiselbeerblätter.'

Alle anderen evaluierenden Attribute werden im Folgenden als Apposition bezeichnet, wobei Apposition definiert wird als »fakultative Konstituente einer Nominalphrase, die syntaktisch und referentiell mit dem nominalen Kern [das heißt dem Kopf, G.J.] übereinstimmt «(Bußmann 2002: 89), verglichen auch Murphy 1968:

If two nouns, or a pronoun and a noun, in succession denote the same person or thing, they are in apposition. (Murphy 1968: 137)

Substantive hingegen, welche im Possessivsuffix am Kopf kodiert sind, stimmen referentiell mit einem zweiten Referenten überein, welcher mit dem ersten in Relation gesetzt wird. Dieses eine Attribut wird in der vorliegenden Arbeit als »Modifizierer» bezeichnet (siehe unten). Des Weiteren kann sogar der Modifizierer zusätzlich attribuiert werden.

(29) NM 2094

$\begin{array}{lllll}\text { tus } & - \text { ən } & \text { ojka } & \text { tus } & -e \\ \text { moustache } & -A D J Z R & \text { old man } & \text { moustache } & - \text { SG }<3 S G\end{array}$

'Bart des bärtigen Alten.'

\subsubsection{Realisierung des Modifizierers}

Der Modifizierer nimmt eine gesonderte Stellung unter den Attributen ein, er ist koreferent mit dem im Possessivsuffix kodierten Referenten und wird auf verschiedene Weise realisiert. »Modifizierer « ist zunächst ein »semantischer Terminus zur Bezeichnung von sprachlichen Ausdrücken, die zusätzliche, von dem modifizierten Ausdruck (Modifikand) nicht geforderte Informationen vermitteln« (Bußmann 2002: 441f.). Unter referentiellen Aspekten unterscheidet man restriktive und nicht-restriktive Modifizierer. Erstere »leisten einen Beitrag zur Festlegung der Referenz des Modifikanden «, während letztere »keinen Einfluss auf die Referenz« haben (Bußmann 2002: 441f.). Formal unterscheidet man unter anderem gebundene Modifizierer (»bound modifier «) - das heißt, die einem Host angefügt werden müssen, zum Beispiel Possessivsuffixe und freie Modifizierer, die entweder Wortgröße (Substantive) oder sogar Satzgröße (in Form von Relativsätzen) haben können (Filchenko 2007: 303). Possessivsuffixe sind demnach gebundene restriktive Modifizierer, welche Einfluss auf die Referenz des Kopfes nehmen, indem sie eine Relation zu einem Referenzpunkt aufbauen. Nominale

90 Hier ist anzumerken, dass ein Beleg im Korpus eine doppelte Realisierung des Modifizierers aufweist, in Form eines Eigennamens sowie eines Personalpronomens (NM_text_001_0067). 
Attribute, welche auf den im Possessivsuffix kodierten Referenten verweisen, sind demnach restriktive freie Modifizierer, da nur Substantive auf Objekte in der außersprachlichen Welt referieren können, Adjektivattribute referieren selbst jedoch nicht. Nur Substantive können daher durch Pro-Formen ersetzt werden, das heißt mit einem Possessivsuffix kodiert werden. Demnach wird der Modifizierer nicht ausschließlich als Substantiv realisiert, Modifizierer erscheinen auch als Personalpronomen (unter anderem als emphatische Personalpronomen) oder werden an der Oberfläche des Satzes gar nicht realisiert, die betreffende Position in der Nominalphrase bleibt leer. In der vorliegenden Arbeit wird diese Leerstelle mit dem Platzhaltersymbol $\emptyset$ repräsentiert (verglichen Kapitel 4.2).

Eine weitere häufige Form der Attribuierung eines Substantives in den ugrischen Sprachen ist eine Partizipialform, welche dem Bezugswort vorangeht (zum Beispiel Murphy 1968: 138). Bei dieser Art der Attribuierung handelt es sich um eine Form des Relativsatzes, das heißt um eine Nebensatzkonstruktion, welche einen Referenten des Hauptsatzes näher bestimmt. Relativsätze werden ebenfalls zu den Modifizierern gezählt und haben die Größe eines Satzes (Givón 2001b: 2). Zwischen Relativsätzen und der Possessivkonstruktion besteht in den ob-ugrischen Sprachen eine strukturelle Ähnlichkeit. Anders als in den temporalen, kausalen oder modalen Nebensatzkonstruktionen, wird nicht das Prädikat mit dem Possessivsuffix versehen, sondern der Kopf. Somit sind Relativsätze eine Unterart der Konstruktion Subs-Px, manche Forschungen sehen in der Relativsatzkonstruktion eine Grammatikalisierung der zwischen Referenzpunkt und Target in der Possessivkonstruktion ausgedrückten Relation (zum Beispiel Nikolaeva 1999a: 85f.). Weiteres zum Relativsatz als Modifizierer siehe im Folgenden.

Nachdem die Abgrenzung zwischen Adjektivattributen und referentiellen Modifizierern erfolgt ist, gilt es abschließend, die Abgrenzung vom Modifizierer in der Nominalphrase und syntaktischer Kernrolle im Satz selbst vorzunehmen. Dieser Schritt ist notwendig, um die tatsächliche pronominale oder lexikalische Erwähnung des Modifizierers gegenüber der Leerstelle in der Konstruktion in Relation zu setzen. Die Typologie der ugrischen Sprachen legt nahe, dass Referenzpunkte, welche gleichzeitig syntaktische Kernrollen ausüben, eher auf Satzebene statt in der Konstruktion pronominal oder lexikalisch erwähnt werden. Dementsprechend wird in der vorliegenden Arbeit ein Referent nur dann als lexikalische oder pronominale Realisierung des Modifizierers gewertet, wenn er eindeutig der Konstruktion zuzuordnen ist. Das bedeutet, dass in Fällen, in welchen der Referenzpunkt gleichzeitig auch Subjekt des Satzes (auch des übergeordneten Satzes) ist, die lexikalische und pronominale Erwähnung des Referenten als syntaktische Kernrolle und als Modifizierer gewertet wurde. Dies lässt sich durch einige Beispiele begründen, in welchen die Zuordnung von syntaktischer Kernrolle und Modifizierer eindeutig ist:

(30) NM_text_001_0070:2

$\begin{array}{lllllllll}\text { mortim } & e k^{\mathrm{w} a} & \text { pawl } & -e & -t & \text { ta } & \text { ol } & -s & - \text {-ry } \\ \text { southland } & \text { woman } & \text { village } & -\mathrm{SG}<3 \mathrm{SG} & -\mathrm{LOC} & \mathrm{EMPH} 1 & \text { live } & -\mathrm{PST} & -3 \mathrm{DU}\end{array}$

'Sie lebten im Dorf der Südland-Frau.'

Der Begriff Modifizierer bezieht sich in der folgenden Arbeit somit ausschließlich auf den Referenzpunkt. Die entsprechende Analysezeile im Korpus ist mit ReFPunkt 
bezeichnet (verglichen Kapitel 4.2). Der Referenzpunkt kann in der Konstruktion demnach in folgenden Realisierungen auftreten:

\begin{tabular}{|c|c|c|c|c|c|}
\hline Typ & Modifizierer & & Kopf & & Vorkommen \\
\hline 1.b.1 & Substantiv & & Substantiv & $-P x$ & Mansisch, Chantisch, Ungarisch \\
\hline 1.b.2 & Pronomen & & Substantiv & $-P x$ & Mansisch, Chantisch, Ungarisch \\
\hline 1.b.3 & $\varnothing$ & & Substantiv & $-P x$ & Mansisch, Chantisch, Ungarisch \\
\hline 1.b.4 & Relativsatz & & Substantiv & $-P x$ & Mansisch, Kazym-Chantisch, Ungarisch \\
\hline 1.c.1 & Substantiv & $-C x$ & Substantiv & $-P x$ & Ungarisch \\
\hline 1.c. 2 & Pronomen & $-C x$ & Substantiv & $-P x$ & Ungarisch \\
\hline
\end{tabular}

Tabelle 15: Realisierungen des Modifizierers in der Konstruktion SuBs-Px im Korpus der vorliegenden Arbeit.

In weit mehr als drei Viertel aller Belege bleibt die Stelle des Modifizierers in der Konstruktion leer. Dies bedeutet, dass der Referenzpunkt in diesen Konstruktionen ausschließlich durch das Possessivsuffix kodiert ist und somit der Referenzpunkt nicht nur vorerwähnt ist, sondern auch eine topikale Rolle im Text innehat. Dies entspricht den Beschreibungen zum Possessivsuffix (zum Beispiel Kulonen 2007: 195). Die zweitgrößte Gruppe bilden die Konstruktionen mit einer lexikalischen Erwähnung des Modifizierers in Form eines Substantivs. Dies ist ebenfalls ein zu erwartendes Ergebnis, da dieser Konstruktionstyp der sogenannten Izafet-Konstruktion entspricht, welche insbesondere im Ungarischen häufig auftritt (zur Izafet-Konstruktion siehe Kapitel 5.2.2.1). Überraschender dabei ist, dass dieser Konstruktionstyp auch im Ob-Ugrischen belegt ist (116 Belege): Den meisten Beschreibungen zum Gebrauch des Possessivsuffixes im Ob-Ugrischen ist zu entnehmen, dass das Possessivsuffix dann in der Konstruktion erscheint, wenn der Referenzpunkt vorerwähnt ist und die Konstruktion unmarkiert bei einer Juxtaposition von zwei Substantiven bleibt (zum Beispiel Nikoaleva 1999: 52; Murphy 1968: 183). Der Konstruktionstyp Subs Subs-Px wird für das Ob-Ugrische an sich nicht beschrieben. Die betreffenden Belege lassen sich in etwa in drei Vorkommen gruppieren: Das Substantiv ist oftmals ein Eigenname (18 Belege), es handelt sich um die Aufzählung mehrerer Referenten aus einer vorerwähnten Gruppe von Referenten, oder es handelt sich hier um eine Teil-Ganzes-Beziehung zwischen beiden Referenten. Letztere Belege werden in Kapitel 5.2.2.2 genauer analysiert, die ersten beiden Vorkommen haben gemeinsam, dass hier Referenten konkret benannt werden oder - wenn der Eigenname nicht genannt wird - es sich trotzdem um spezifische bekannte Individuen handelt (verglichen Loos (Hg.) 2004c).

(31) NM 2089

nilit xum nam -e

fourth man name $-S G<3 S G$

'Der Name des vierten Mannes.'

Die Belebtheit des Referenten scheint somit ebenfalls eine Auswirkung auf die Realisierung des Modifizierers zu haben. 
Die drittgrößte Gruppe bilden die Konstruktionen mit Modifizierer in pronominaler Erwähnung. Auch diese ist in allen ugrischen Sprachen belegt. Eine pronominale Erwähnung kann dabei in Form eines Personalpronomens erfolgen (dies ist das häufigste Pronomen zur Realisierung des Referenzpunktes, 113 Belege). Hier sind nahezu alle Personen und Numeri des Possessivsuffixes zusammen mit dem zugehörigen Personalpronomen belegt. Viele Abhandlungen beschreiben das zusätzliche Erscheinen eines Personalpronomens als Betonung (zum Beispiel Kulonen 2007: 195). Damit ließe sich auch das Erscheinen der emphatischen Form des Personalpronomens im Mansischen erklären.

(32) NM_text_007_0035:1

$\begin{array}{lllllll}a^{j} & \text { nanki } & \text { kol } & - \text {-2n } & \text { xot- } & \text { s'aras } & \text {-awe } \\ \text { now } & \text { 2SG.EMPH } & \text { house } & - \text { SG }<2 \text { SG } & \text { PERF } & \text { burn } & \text {-PASS[PRS.3SG] }\end{array}$

'Jetzt wird dein Haus niederbrennen.'

Pronominale Formen sind weiterhin Reziprok- und Reflexivpronomen, das ungarische Anredepronomen kelmed (archaisch, heute eher ön,Sie') oder die Indefinitpronomen mansisch xotpa ,jemand', Kazym-Chantisch xojat ,id.' und ungarisch valaki ,id.. Letztere sind ebenfalls als Referenzpunkt eines Possessivsuffixes in der Konstruktion belegt, obwohl offensichtlich der konkrete Referent nicht bekannt ist.

Damit scheint - möglicherweise wieder beschränkt auf belebte Referenten - die Vorerwähnung allein auszureichen, das heißt, das Wissen, dass es einen Referenten gibt und über diesen im Verlauf gesprochen wurde, ohne dass eine konkrete Person in der außersprachlichen Welt zugeordnet werden kann. Da aufgrund der geringen Anzahl der Vorkommen keine weiteren Aussagen über die Verwendung getroffen werden können, werden die Belege mit Indefinitpronomen nicht weiter analysiert.

Die kleinste Gruppe bilden die Konstruktionen mit Relativsatz als Realisierung des Modifizierers. Genauer handelt es sich dabei um einen Partizipialsatz, das heißt einen subordinierten Nebensatz, welcher dem Satzglied vorangeht, welches modifiziert wird. Im Partizipialsatz wiederum können Subjekt, direktes und indirektes Objekt sowie adverbiale Ergänzungen erscheinen. Der Kopf übt dabei zwei syntaktische Rollen aus, sowohl eine im Haupt- als auch eine im Nebensatz. Der im Possessivsuffix kodierte Referent steht zum Kopf in einer Relation, welche im Partizip Perfekt ausgedrückt wird - die syntaktischen Rollen im Nebensatz werden entsprechend nach der Verbalhandlung verteilt. Der Referenzpunkt selbst kann wiederum als Leerstelle, Pronomen oder lexikalische Erwähnung erscheinen. Die geringe Anzahl der Belege lässt hier jedoch keine weiteren aussagekräftigen Schlüsse zu.

(33) NM_text_009_0080:1

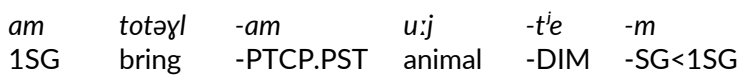

'Mein Tierchen, das ich gebracht habe.'

Diese Form der Relativsatzkonstruktion ist typisch für das Ob-Ugrische, im Ungarischen wird stattdessen mit einem Relativpronomen gearbeitet, welches im Relativsatz als Platzhalter für das Satzglied steht, welches zu modifizieren ist und demselben folgt (zum Beispiel Beispiel 37). Ein Beleg im Korpus jedoch entspricht der ob-ugrischen Relativsatzkonstruktion mit Possessivsuffix: 
(34) HU_text_009_0268

\begin{tabular}{|c|c|c|c|c|c|c|c|c|c|}
\hline $\begin{array}{l}a \\
\text { the }\end{array}$ & $\begin{array}{l}\text { rosdá } \\
\text { rust }\end{array}$ & $\begin{array}{l}-s \\
-A D J Z R\end{array}$ & $\begin{array}{l}\text { puska } \\
\text { shotgun }\end{array}$ & $\begin{array}{l}e z \\
\text { this }\end{array}$ & $\begin{array}{l}\text {-en } \\
\text {-SUP }\end{array}$ & $\begin{array}{l}\text { pedig } \\
\text { and }\end{array}$ & $\begin{array}{l}a \\
\text { the }\end{array}$ & $\begin{array}{l}\text { madzag } \\
\text { string }\end{array}$ & $\begin{array}{l}-r a \\
-S U B\end{array}$ \\
\hline $\begin{array}{l}\text { köt } \\
\text { to tie }\end{array}$ & $\begin{array}{l}-o ̈ t t \\
\text {-PST }\end{array}$ & $\begin{array}{l}-0 \\
-3 S G\end{array}$ & $\begin{array}{l}\text { vereb } \\
\text { sparrow }\end{array}$ & $\begin{array}{l}-e ́ \\
-S G<3 S G\end{array}$ & $\begin{array}{l}-t \\
-A C C\end{array}$ & $\begin{array}{l}\text { lobál } \\
\text { to dangle }\end{array}$ & $\begin{array}{l}-t \\
-P S T\end{array}$ & $\begin{array}{l}-a \\
-S G<3 S G\end{array}$ & \\
\hline
\end{tabular}

'Die rostige Flinte, an der, mit Bindfaden angebunden, der Sperling hin und her geschwenkt wurde.'

Über die tatsächliche Häufigkeit dieser Konstruktion lässt sich aufgrund der Suchabfrage ebenfalls mithilfe des HNC keine Aussage treffen ${ }^{91}$, es ist jedoch möglich, einige Beispiele zu finden, sodass der Beleg in Korpus der vorliegenden Arbeit durchaus einer im Ungarischen gebräuchlichen Konstruktion entspricht:

(35) Ungarisch, HNC_Abfrage_0041.pdf

$\begin{array}{lllllll}\text { Dolores } & j o ́ & -I & \text { ismer } & -t & \text { dallam } & -a \\ \text { D. } & \text { good } & \text {-ADVZR } & \text { to know } & \text {-PST[3SG] } & \text { melody } & -S G<3 S G\end{array}$

'Die von Dolores gut gekannte Melodie.'

Abschließend sei auf einige Belege des Ungarischen hingewiesen, welche als Modifizierer eine Numeralie beinhalten.

(36) HU_text_005_0071

már hét álló esztende -je

already seven whole year $\quad-S G<3 S G$

'Jetzt sind es schon volle sieben Jahre.'

Hierfür gibt es insgesamt vier Belege im Korpus der vorliegenden Arbeit. Eine Suche im HNC mit derselben Struktur (Modifizierer: Numerale, Kopf: esztendő ,Jahr') ergibt insgesamt 11.434 Belege (60,93 Vorkommen pro einer Millionen Wörter, HNC_ Abfrage_0011.pdf) für diese Konstruktion. Eine Suche nach dieser Konstruktion mit dem Possessivsuffix der dritten Person Singular ergibt 3.286 Belege (17,51 Vorkommen pro einer Millionen Wörter, HNC_Abfrage_0013.pdf). Das Lemma esztendő kommt insgesamt 4.777 mal (25,46 Vorkommen pro einer Millionen Wörter HNC_Abfrage_0012. pdf) possessivsuffigiert vor. Damit scheint die Konstruktion Num Subs esztendö $_{-} \mathrm{Px}$ fast drei Viertel aller possessivsuffigierten Formen des Lemmas esztendő auszumachen, die Konstruktion Num Subs-Px scheint eine Art Quantifizierung auszudrücken, wird jedoch mangels weiterer Belege nicht weiter in der Analyse berücksichtigt.

Zuletzt soll noch auf das dependent-marking in der ungarischen Possessivkonstruktion eingegangen werden. Die Realisierung des Modifizierers mit dependent-marking kann sowohl als Substantiv wie auch als Pronomen erfolgen - was der Beschreibung in den Quellen entspricht, dass es sich hier ebenfalls um eine Art der Betonung insbesondere in Form einer Possessor-Extrahierung handelt (zum Beispiel Kenesei/ Vago/Fenyvesi (Hg.) 1998): Je größer die morpho-phonologische Realisierung, desto fokussierter das Element. Neben Substantiven und Personalpronomen können auch Relativpronomen und Demonstrativpronomen sowie Pro-Adjektive mit der DativMarkierung versehen werden. Der sogenannten Possessor-Extrahierung entspricht die veränderte Satzstellung gegenüber der Possessivkonstruktion im Allgemeinen:

91 Es war lediglich möglich, eine Suchabfrage nach der Partizip Perfekt Form direkt gefolgt von einem possessivsuffigierten Substantiv durchzuführen, weswegen ein relativ umfangreiches Ergebnis zustande kommt. 
Zwischen Referenzpunkt und Target können andere Satzglieder erscheinen, dies ist im Korpus bei den jeweiligen Belegen mit Auslassungs-Klammern angezeigt.

(37) HU 0730

$\begin{array}{lllll}\text { mely } & -n e k(. .) & a & \text { virág } & -a \\ \text { which } & \text {-DAT } & \text { the } & \text { flower } & -\mathrm{SG}<3 S \mathrm{SG}\end{array}$

'Deren Blüte.'

Erwähnenswert ist in diesem Zusammenhang die Korrelation zwischen dependentmarking und Determinierern in der Konstruktion: Nahezu alle Targets in Konstruktionen mit dependent-marking am Referenzpunkt sind mit dem definiten Artikel, seltener dem Demonstrativpronomen, versehen. Einige Forschungen sehen den definiten Artikel somit als Platzhalter innerhalb der Possessivkonstruktion für den extrahierten Possessor an (zum Beispiel Szabolcsi 1987; Szabolcsi 1994). In der vorliegenden Arbeit entsprechen die Belege des dependent-marking im Ungarischen jedoch nur einem geringen Teil der Vorkommen, sodass nicht weiter gesondert auf sie eingegangen wird.

Des Weiteren sei hier auf die ungarische Habeo-Konstruktion verwiesen: Diese entspricht in ihrer Struktur ebenfalls der Konstruktion Subs-Px, wenngleich sie in den meisten Abhandlungen aufgrund des Kopulaverbs zu den intransitiven, das heißt prädikativen Possessivkonstruktionen gezählt wird (verglichen Kapitel 1.2). Die vorliegende Arbeit zählt die Habeo-Konstruktion zum Konstruktionstyp Subs-Px, zieht sie jedoch nicht weiter in die Analyse mit ein, da sie eine grammatikalisierte Form der Konstruktion Subs-Px darstellt und das Possessivsuffix damit obligatorischer Bestandteil ist. Somit unterliegt die Habeo-Konstruktion nicht denselben informations-strukturellen Faktoren wie andere Vorkommen der Konstruktion SuBs-Px und kann nicht zum Zwecke der Analyse in der vorliegenden Arbeit herangezogen werden. Es sei jedoch darauf hingewiesen, dass lexikalisch oder pronominal erwähnte Referenzpunkte der Habeo-Konstruktion ausschließlich mit dependent-marking auftreten. Sie machen den Großteil der Vorkommen mit dependent-marking im Korpus der vorliegenden Arbeit aus. Das dependent-marking wird weiterhin als Ausdruck der Grammatikalisierung dieser Konstruktion angesehen (Kenesei/Vago/Fenyvesi (Hg.) 1998: 157). Zu den grammatikalisierten Konstruktionen wird neben der Habeo-Konstruktion auch die Nezessiv-Konstruktion des Ungarischen gezählt:

\begin{tabular}{llll}
\hline Adverbiale Bestimmung & Kopulaverb & Subjekt & Konstruktion \\
\hline a fiú-nak & nincs & kedv-e & Habeo-Konstruktion \\
a kertészem-nek bojtárra & van & szükség-e & Nezessiv-Konstruktion \\
\hline
\end{tabular}

Tabelle 16: Konstruktionen des Ungarischen mit obligatorischer Verwendung des Possessivsuffixes.

Auch das Ob-Ugrische verfügt über eine Habeo-Konstruktion mit ähnlicher Struktur, viele Quellen gehen jedoch davon aus, dass diese nicht mehr produktiv ist (zum Beispiel Kangasmaa-Minn 1984: 121). Da aus diesem Grund nichts über den Grad der Grammatikalisierung ausgesagt werden kann, erhalten Belege aus dem Korpus, welche der Struktur der Habeo-Konstruktion ähneln, zwar einen dahingehenden Vermerk, werden jedoch nicht aus der weiteren Analyse ausgeschlossen. 


\subsubsection{In der Konstruktion auftretende Determinantien}

Wie in Kapitel 3.4 erwähnt können im Ugrischen in der Possessivkonstruktion auch Determinierer auftreten. Für die vorliegende Arbeit sind hier insbesondere zwei Determinierer von Relevanz, der definite Artikel des Ungarischen sowie Demonstrativpronomina in allen ugrischen Sprachen. Bei letzteren ist zu unterscheiden, ob sie auf den Modifizierer referieren - und damit eine weitere Realisierungsmöglichkeit desselben darstellen (Beispiel 38) - oder, ob sie ebenfalls den Kopf attribuieren (Beispiel 39), was häufiger vorkommt.

(38) HU 951

$\begin{array}{lllllll}\text { Ez } & -e k & -n e k & a & k r a ́ t e r & -e ́ & -b e n \\ \text { this } & -\mathrm{PL} & \text {-DAT } & \text { the } & \text { caldera } & \text {-SG<3SG } & \text {-INE }\end{array}$

'In deren Kratern.'

(39) NM_text_008_0181:4

ma:n ti xum -juw a:tim ke o:l -s

$1 \mathrm{PL}$ this man $-\mathrm{SG}<1 \mathrm{PL}$ does not exist if be -PST[3SG]

'If it hadn't been for this man.'

Der Fokus der vorliegenden Arbeit ist nicht auf die Bestimmung der Funktionen von Determinantien gerichtet, sondern auf die Ko-Okkurrenz von Possessivsuffix und Determinans im jeweiligen Umfeld des Auftretens. Das Ungarische ist eine der wenigen uralischen Sprachen, welche über einen bestimmten Artikel verfügt, er tritt in zwei Formen auf - $a$ bzw. $a z$ vor vokalisch anlautenden Substantiven - und hat seinen Ursprung im distalen Demonstrativpronomen az (Molnár 2014: 32).

(40) HU 0027

az ap -ja

the father $-\mathrm{SG}<3 \mathrm{SG}$

'Sein/Der Vater.'

Als Determinans kann weiterhin das Demonstrativpronomen in der Konstruktion erscheinen. Die ugrischen Sprachen verfügen über jeweils zwei Demonstrativpronomina: ein proximales und ein distales. Im Mansischen sind dies $t i$, diese,r,s' und $t a$,jene,r,s. Im Chantischen $t^{j i}$, te: $m i$, diese,r,s' und $t^{j} u_{\text {i. }}$, tom, jene,r, s' (Surgut-Chantisch) bzw. si $i^{\prime}$ diese,r,s ${ }^{c}$ tam ,id.' und tom ,jene,r,s' (Kazym-Chantisch) und im Ungarischen $e z$, diese,r,s' und $a z$ ,jene,r,s. Dem Demonstrativpronomen, welches als Attribut mit dem Kopf kongruiert, folgt im Ungarischen der bestimmte Artikel, prädikativ hingegen nicht:

(41) HU 0815

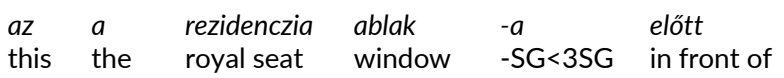

'Vor dem Fenster der königlichen Residenz.'

In Relativsätzen steht das Vorkommen von Demonstrativpronomen oder definitem Artikel in Zusammenhang mit der Vorerwähntheit des Referenten: Ist dieser vorerwähnt, so kann eine Zusatzinformation mit einem nicht-restriktiven Relativsatz ausgedrückt werden. Ist der Referent hingegen neu und nicht vorerwähnt, ist die Information im Relativsatz notwendig zur Eingrenzung des Referenten (restriktiver Relativsatz). Ersterer Typ kann einen definiten Artikel enthalten, während letzterer das Demonstrativpronomen verlangt (Molnár 2014: 46). Typisch sind Possessivkonstruktionen in restriktiven Relativsätzen daher im frame-setting zu Beginn einer Erzählung: 
(42) HU_text_002_0001

\begin{tabular}{|c|c|c|c|c|c|c|c|c|c|c|c|}
\hline $\begin{array}{l}\text {-szer } \\
\text {-ITER }\end{array}$ & $\begin{array}{l}\text { vol } \\
\text { to be }\end{array}$ & $\begin{array}{l}-t \\
- \text { PST }\end{array}$ & $\begin{array}{l}-0[\ldots] \\
-3 S G[\ldots]\end{array}$ & $\begin{array}{l}\text { egy } \\
\text { one }\end{array}$ & $\begin{array}{l}\text { folyó } \\
\text { river }\end{array}$ & $\begin{array}{l}\text { en } \\
\text { this }\end{array}$ & $\begin{array}{l}\text {-nek } \\
\text {-DAT }\end{array}$ & $\begin{array}{l}a \\
\text { the }\end{array}$ & $\begin{array}{l}\text { part } \\
\text { shore }\end{array}$ & $\begin{array}{l}-j a ́ \\
-S G<3 S G\end{array}$ & $\begin{array}{l}-n \\
-S U P\end{array}$ \\
\hline $\begin{array}{l}-t \\
-P S T\end{array}$ & $\begin{array}{l}-0 \\
-35\end{array}$ & & $\begin{array}{l}\text { vén } \\
\text { old }\end{array}$ & $\begin{array}{l}\text { vas } \\
\text { llow }\end{array}$ & $\begin{array}{l}\text { füzfo } \\
\text { willov }\end{array}$ & & & & & & \\
\hline
\end{tabular}

'Es war einmal, der Himmel weiss wo (...) ein Fluss, und am Ufer des Flusses eine alte hohe Weide.'

Weitere Bestandteile, welche in diesem Zusammenhang Berücksichtigung finden, sind sämtliche Pronomina und Numeralia, welche ebenfalls eine Vorerwähnung voraussetzen. Hierunter fallen zum Beispiel Fragepronomina wie mansisch mana, welche,r,s und chantisch muj (kem), id.' oder Numeralia wie ungarisch egyik, der/die/das eine‘. Im Ob-Ugrischen haben die Numeralia zwar nur eine Form, jedoch scheint die Verwendung von mansisch $a k^{w}$,eine,r,s' und Surgut-Chantisch $e j$,id.' eine vergleichbare Funktionen übernehmen zu können.

Des Weiteren ist es möglich, mit Ordinalia zu arbeiten, zum Beispiel mansisch $x u r$ mit ,dritte,r,s, oder Distributiva, zum Beispiel ungarisch mind a két ,beide. ${ }^{\text {(92 }}$ Auch die Pronomina mansisch mot , andere,r,s' und Kazym-Chantisch $i$,id.' und Surgut-Chantisch tom ,id. ${ }^{93}$ und das distale adjektivische Demonstrativpronomen olyan, solche,r,s ${ }^{c}$ werden zu den Determinierern gezählt. Alle hier erwähnten Formen sind im Korpus belegt, dort sind sie als ProNum respektive PROADj kodiert.

Abschließend ist zusammenzufassen, dass die Nominalphrase durch verschiedene Attribute erweitert werden kann, welche auch gemeinsam auftreten können: Modifizierer, Determinierer und adverbiale Appositionen. Schematisch lässt sich die Konstruktion Subs-Px wie folgt darstellen:

\begin{tabular}{|c|c|c|c|}
\hline \multicolumn{4}{|l|}{ Nominalphrase } \\
\hline \multicolumn{3}{|l|}{ Attribut } & \multirow[t]{2}{*}{ Kopf } \\
\hline Determinierer & Modifizierer & Appositionen & \\
\hline $\boldsymbol{\nabla}$ & $\nabla$ & $\nabla$ & $\nabla$ \\
\hline tiu: & ke:njer qo: & $0: \beta$ & to $\beta-\partial t$ \\
\hline that & poor man & head & bone $S G<3 S G$ \\
\hline
\end{tabular}

Tabelle 17: Schematische Darstellung der Bestandteile der Possessivkonstruktion.

92 Die Form mind a két, welche im Korpus belegt ist, ist verhältnismäßig selten zu finden (max. 2.002 Belege im HNC, das heißt 10,67 Vorkommen pro einer Millionen Wörter, eine genauere Bestimmung ist durch die Abragemethode des HNC nicht möglich. HNC_Abfrage_0042.pdf). Das Pendant mindkét ist das häufigere Distributivpronomen (17.095 Belege im HNC das heißt 91,10 Vorkommen pro einer Millionen Wörter, HNC_Abfrage_0043.pdf).

93 Hier sei auch noch auf die Formgleichheit im Surgut-Chantischen zwischen tom, jene,r,s und tom, andere, r, s hingewiesen. 
Für die Analyse in der vorliegenden Arbeit sind die ersten beiden Attribuierungen (Determinierer und Modifizierer) von Relevanz und werden deshalb in der Korpusanalyse gekennzeichnet; Appositionen wurden nicht gesondert vermerkt (verglichen Kapitel 4.2).

\subsubsection{Lesart der Relation zwischen Modifizierer und Kopf}

Zuletzt soll noch auf die Relation zwischen Referenzpunkt und Target eingegangen werden. Wie im Forschungsdesign der Arbeit erwähnt soll dabei nicht der Ausdruck von Besitz im Fokus stehen. Dennoch ist der Ausdruck einer Relation zwischen zwei Entitäten eine der grundlegenden Eigenschaften der Possessivkonstruktion und darf deshalb nicht gänzlich unerwähnt bleiben. Grundsätzlich lassen sich mit der Possessivkonstruktion der ugrischen Sprachen sämtliche in Kapitel 1.2.2.1 beschriebenen Default-Lesarten (Eigentum, Teil-Ganzes, Verwandtschaftsverhältnisse, Assoziation) wie in den meisten Sprachen der Welt (Aikhenvald 2012a: 3) ausdrücken.

Für das Ungarische zum Beispiel beschreibt Kenesei/Vago/Fenyvesi (Hg.) 1998 folgende Relationen: Besitz, Verwandtschaft, Teil-Ganzes, dazu noch Ursprung, Relationen zwischen Agens und Objekt einer Handlung, aber auch Maße, Eigenschaften, nominalisierte Handlungen, Identität und Ad-hoc-Relationen. Die Gruppierung unterteilt die Relationen in solche, die auch in der lokalen Habeo-Konstruktion ausgedrückt (die sogenannten prototypischen Possessivkonstruktionen, in der Tabelle mit * gekennzeichnet), und solche, die nicht umgewandelt werden können (verglichen Kenesei/Vago/Fenyvesi (Hg.) 1998: 160):

\begin{tabular}{lll}
\hline Relation & Beispiel & Übersetzung \\
\hline possession* & Péter verse & ,Peters Gedicht' \\
\hline relation* $^{*}$ & Péter húga & ,Peters Schwester' \\
\hline part-whole* & az asztal lába & ,Der Fuß des Tisches' \\
\hline measure & a tej literje & ,Ein Liter Milch' \\
\hline source* & a vad nyoma & ,Die Spur des Wildes' \\
\hline property & a diadal mámora & ,Der Rausch des Sieges' \\
\hline action nominalization & Péter vizsgálása & ,Peters Prüfung' \\
\hline agent-object* & Péter üldözöje & ,Peters Verfolger' \\
\hline identity & London városa & ,Die Stadt London' \\
\hline ad hoc relation & Péter minisztere & ,Peters Minister' \\
\hline
\end{tabular}

Tabelle 18: In der Possessivkonstruktion des Ungarischen ausgedrückte Relationen nach Kenesei/Vago/ Fenyvesi (Hg.) 1998: 160.

Die Relation »measure« (Maß) ist im Korpus der vorliegenden Arbeit nicht belegt, eine vergleichbare Suche im HNC jedoch ergibt für das Lemma liter ,Liter` insgesamt 
4.589 Vorkommen (24,46 Vorkommen pro einer Millionen Wörter,HNC_Abfrage_0020. $\mathrm{pdf}^{94}$ ). Eine Abfrage nach dem Lemma liter mit Possessivsuffix ergibt insgesamt 253 Belege (1,35 Vorkommen pro einer Millionen Wörter, HNC_Abfrage_0021.pdf). Von diesen 253 Belegen kommen circa 160 Token in der Konstruktion Subs Subs-Px vor, davon nur circa zehn ohne einen Determinierer. Ein Pronomen geht lediglich in 14 Fällen dem Kopf voran, darunter ein einziges Personalpronomen, in der ersten Person Plural (a mi 580 literünkkel szemben ,Gegenüber unseren 580 Litern' HNC_ Abfrage_0021.pdf Beleg Nr. 140). Beim Rest handelt es sich um das Reflexivpronomen maga oder die Pro-Numeralie minden, alle. In rund 59 Fällen erscheint der Referenzpunkt als Leerstelle. Einige Belege konnten nicht berücksichtigt werden, da es sich dabei zum Beispiel um fehlerhafte Annotationen oder fremdsprachliche Belege handelte.

Eine detailliertere Analyse kann aufgrund der Suchabfrage und der Ausgabe der Suchergebnisse nicht erfolgen, so ist diese Analyse beispielhaft für die Realisierung des Modifizierers bei einer Relation vom Typ »measure« anhand des Lemmas liter zu verstehen. Es ist zu erwarten, dass im Großteil der Konstruktionen der Referenzpunkt als Substantiv realisiert wird, der zudem mit einem definiten Artikel determiniert ist und vermutlich nicht vorerwähnt. Diese Belege repräsentieren wahrscheinlich daher die Izafet-Konstruktion (siehe Kapitel 5.2.2.1).${ }^{95}$ Die Relationen "property « und »action nominalization « sind im Korpus belegt, werden dort jedoch anders kategorisiert und beschrieben (siehe bei Person Abstrakt und Person Resultat).

Die Relation »identity« ist im eigenen Korpus nicht belegt, ein vergleichbare Suche im HNC ergibt für das Lemma város ,Stadt' insgesamt 98.483 Belege (524,84 Vorkommen pro einer Millionen Worte, HNC_Abfrage_0022.pdf), davon sind 10.357 Belege (55,19 Vorkommen pro einer Millionen Worte, HNC_Abfrage_0023.pdf) mit Possessivsuffixen versehen. Die Suche nach der Konstruktion Subs Subs ${ }_{\text {város }}$ ergibt insgesamt 7.271 Belege (38,75 mal pro einer Millionen Wörter, HNC_Abfrage_0014.pdf), die Konstruktionen ohne und mit Possessivsuffix sind dabei in etwa gleich verteilt (die HNC_Abfrage_0024.pdf liefert 3380 Belege, 18,01 Vorkommen pro einer Millionen Wörter). Allerdings ist es hier nicht möglich, genauer zu bestimmen, wobei es sich um Possessivkonstruktionen und wobei lediglich um die Abfolge verschiedener Satzglieder handelt. In dieser Größenordnung ist es weiterhin nicht möglich, anhand der vorgegebenen Möglichkeiten der Suchabfrage, gezielt nach pronominalen Erwähnungen zu suchen und aus der Differenz von lexikalischer und pronominaler Realisierung die Konstruktionen mit Leerstelle für den Modifizierer zu ermitteln. Stattdessen kann jedoch die kodierte Person mit herangezogen werden, um eine Einschätzung vornehmen zu können, wie viele der possessivsuffigierten Formen dem Typ London városa entsprechen und wie häufig er im Vergleich zu anderen possessivsuffigierten Formen des Lemmas város ist bzw. ob die Relation »identity« die häufigste Ausdrucksart mit dem Lemma város ist. Die Possessivsuffixe der ersten Personen machen 1.952 Belege (10,40 Vorkommen pro einer Millionen Wörter; HNC_Abfrage_0026.pdf) aller possessivsuffigierten Vorkommen von város aus. Die Possessivsuffixe der zweiten Person sind

94 Die Trefferzahlen und Prozentsätze entstammen den Angaben des HNC, die den Suchergebnissen mitgeliefert werden.

95 Die Analyse ist in der HNC_Abfrage_0021.pdf zusammengefasst. 
nur 63 mal belegt (0,34 Vorkommen pro einer Millionen Wörter; HNC_Abfrage_0027. pdf). Die dritten Personen kommen 8.342 mal vor (44,46 Vorkommen pro einer Millionen Wörter; HNC_Abfrage_0028.pdf), wovon 7.024 Belege (37,43 Vorkommen pro einer Millionen Wörter; HNC_Abfrage_0029.pdf) auf den Einbesitz dritte Person Singular entfallen.

Von diesen 7.024 Belegen entsprechen 2.818 Token (15,02 Vorkommen pro einer

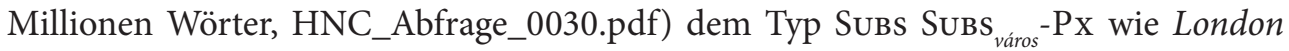
városa; was in etwa einem Viertel aller possessivsuffigierten Formen des Lemmas város im HNC entspricht.

Der Relationstyp "ad hoc relation« ist nicht genauer spezifiziert, dabei handelt es sich vermutlich um Konstruktionen, in welchen zwei Referenten aufgrund des Handlungsverlaufes miteinander in Relation gesetzt werden. Solche Relationen sind im Korpus der vorliegenden Arbeit ebenfalls für das Ungarische belegt, werden jedoch ebenfalls anders beschrieben und eingeordnet.

Für die Analysezwecke der vorliegenden Arbeit wurden weiterhin die vier DefaultRelationen angepasst. Im Fokus standen dabei insbesondere die (Un)-Belebtheit des Referenten und Teil-Ganzes-Beziehungen, was sich auch anhand der Daten bestätigt hat. So wurde in erster Linie die Person berücksichtigt, eine Untergruppe bilden dabei Lebewesen. Dies begründet sich damit, dass einerseits die Belebtheit dieser beiden beispielsweise gegenüber Gegenständen herausgestellt werden sollte. Zudem scheint aber auch noch eine gewisse Abweichung zwischen Personen und anderen Lebewesen zu existieren. Diese Belebtheit wurde auch in Bezug auf Teil-Ganzes-Beziehungen mit entsprechenden Untergruppen berücksichtigt. Eine vierte Gruppe bilden Abstrakta. Somit kommt auch die vorliegende Arbeit nicht ohne einen Begriff aus, der so unbestimmt ist, dass eine Vielzahl von Relationen damit erfasst werden kann. Dies liegt zum einen daran, dass die Relation zwischen zwei Referenten in einer Possessivkonstruktion durch die fehlende Verbalisierung derselben immer einer gewissen subjektiven Interpretation unterliegt. Zudem wird die attributive Possessivkonstruktion in den meisten uralischen Sprachen weitaus häufiger und zum Ausdruck von weit mehr Relationen eingesetzt als zum Beispiel in indo-europäischen Sprachen, welche über weitere Possessivkonstruktionen verfügen und teilweise Restriktionen in der Kombination von Konstruktion und ausgedrückter Relation beinhalten. So fällt auch der Verfasserin mitunter die Bestimmung einiger Relationen schwer. Es wurde dennoch versucht, die Belege, welche zu dieser Gruppe zählen, so gering wie möglich zu halten, unter anderem auch dadurch, dass die leichter bestimmbaren Relationen möglichst genau beschrieben wurden. Die Begriffe, welche dem Referenzpunkt und dem Target zugeordnet werden können, sind somit Person, Gegenstand, Ganzes/Teil (von), Abstrakt, Lokalisation und Resultat. Die Relation ergibt sich aus der Kombination der jeweiligen dem Referenzpunkt und Target zugeordneten Begriffe mit einer Tilde zwischen den beiden, zum Beispiel Person Gegenstand für Eigentumsverhältnisse.

Die Reihenfolge der Benennung entspricht dabei der Reihenfolge von Modifizierer und Kopf in der Konstruktion; das heißt, der erste Begriff bezieht sich auf den Referenzpunkt, der zweite auf das Target. Sie sind demnach genau entgegengesetzt der Reihenfolge in der Glossierung der Possessivsuffixe. Prinzipiell besteht bei der Relation zwischen Referenzpunkt und Target keine Einschränkung, es zeichnen sich jedoch 
deutliche Präferenzen für bestimmte Relationen ab, diese werden im Folgenden genauer beschrieben:

\section{Person Person}

Diese Relation entspricht der Default-Lesart als Verwandtschaftsverhältnis und beinhaltet alle sowohl blutsverwandten als auch angeheirateten Familienverhältnisse.

(43) HU 0878

feleség -em

spouse $\quad-S G<1 S G$

'Meine Ehefrau.'

Verwandtschaftstermini werden häufig nur in einer Possessivkonstruktion beschrieben, und auch in den ugrischen Sprachen gibt es Beobachtungen, dass Nomen, welche enge Verwandtschaftsverhältnisse ausdrücken, nur in ihrer possessiven Form auftreten können (zum Beispiel Filchenko 2007, Murphy 1968). Im Ungarischen sind bei bestimmten Verwandtschaftstermini (Vater, Mutter, jüngerer Bruder, älterer Bruder, jüngere Schwester) oftmals die Possessivsuffixe nicht mehr ohne Weiteres vom Stamm segmentierbar, insbesondere in der dritten Person Singular (statt Wechsel des Endvokals von $a \sim a ́$ bzw. $e \sim e ́$ und Anfügen des Possessivsuffixes der dritten Person Singular mit Bindekonsonant -j- wird der Auslautvokal des Stamms mit diesem Allomorph ersetzt: apa ,Vater ‘ apja ,Vater.SG<3SG` statt *apája). Eine weitere Ausnahme bildet öcs, jüngerer Bruder', hier wird der auslautende Konsonant geminiert: öccse ,Bruder.SG $<3 \mathrm{SG}^{\star}$ statt *öcséje. Laut Kenesei/Vago/Fenyvesi (Hg.) 1998 sind Formen ohne Possessivsuffixe nicht grammatisch (Kenesei/Vago/Fenyvesi (Hg.) 1998: 160). Auch im Korpus der vorliegenden Arbeit sind ausschließlich possessivsuffigierte Formen dieser Lemmata zu finden. Eine Suchanfrage im HNC liefert jedoch Belege ohne Possessivsuffixe, wenn auch in geringem Umfang: Zum Beispiel für húg ,jüngere Schwester sind von 2.373 Vorkommen (12,65 mal pro einer Millionen Wörter) (HNC_Abfrage_0006.pdf), wovon 102 Belege (0,54 mal pro einer Millionen Wörter) (HNC_Abfrage_0007.pdf ${ }^{96}$ ) nicht possessivsuffigiert sind.

Bei bátya ,älterer Bruder insgesamt 5.576 Vorkommen (29,72 mal pro einer Millionen Wörter, HNC_Abfrage_0004.pdf), wovon 135 Belege (entspricht 0,72 mal pro einer Millionen Wörter) nicht possessivsuffigiert sind (HNC_Abfrage_0005.pdf). Bei Bei öcs, jüngerer Bruder' sind 4.486 Vorkommen belegt (23,91 mal pro einer Millionen Wörter, HNC_Abfrage_0008.pdf), ohne Possessivsuffix lediglich acht Belege (0,04 mal pro einer Millionen Wörter, HNC_Abfrage_0009.pdf). Am häufigsten jedoch können apa ,Vater' und anya ,Mutter' ohne Possessivsuffix erscheinen (10.195 von insgesamt 41.911 Vorkommen von apa sind nicht possessivsuffigiert - HNC_Abfrage_0031.pdf und _0032.pdf, sowie 12.445 - von 40.551 Vorkommen für anya; HNC_Abfrage_0033.

96 Die beiden jeweiligen Trefferzahlen zwischen Vorkommen mit Px und ohne Px können nur als Richtwert den Unterschied verdeutlichen, ihnen liegt keine Berechnung zugrunde. Zieht man beispielsweise von 2373 Belegen für das Lemma húg, jüngere Schwester' die 102 Belege für das Lemma húg'id.' ohne Possessivsuffigierung ab, ist die Anzahl an possessivsuffigierten Belegen nicht zwangsläufig 2271, es kann eine geringe Diskrepanz auftauchen (tatsächliches Ergebnis: 2268, HNC_Abfrage_0010.pdf). Dies ist der Annotationsweise des HNC geschuldet. 
pdf und _0034.pdf). Die vorliegende Arbeit bezieht die Verwandtschaftstermini des Ungarischen mit in die Analyse ein; das heißt, die Possessivsuffigierung wird nicht als ausschließlich obligatorisch und ihr Vorkommen daher zu einem gewissen Grad informations-strukturell bedingt angesehen. Grundlage der Entscheidung bilden erstens die Ergebnisse des HNCs und zweitens die Einträge im Wörterbuch: Dort sind die possessivsuffigierten Formen nicht als eigene Lemmata aufgelistet, sondern als Teil des Eintrages zur absoluten Form (zum Beispiel Hessky (Hg.) 2002: 42). Folgende Termini sind im Korpus belegt:

\begin{tabular}{|c|c|c|c|}
\hline Lemma & Formen mit Px & Übersetzung & Belege \\
\hline apa & apám/apád/apja & ,Vater' & $\begin{array}{l}(0010)(0031)(0034)(0040)(0042)(0156) \\
(0286)(0289)(0291)(0399)(0492)(0500) \\
(0673)(0692)(1010)\end{array}$ \\
\hline atya & atyám/atyja & ,Vater' & (0979) (0988) (1018) (1019) \\
\hline anya & anyám/anyád/anyja & ,Mutter' & $\begin{array}{l}(0053)(0055)(0057)(0064)(0066)(0068) \\
(0077)(0079)(0081)(0102)(0303)(0334) \\
(0398)(0674)(0691)(0995)(1004)(1007)\end{array}$ \\
\hline bátya & bátyám/bátyja & ,älterer Bruder' & (0023) (0526) (0555) (0576) \\
\hline öcs & ecsém/(öccse) & ,jüngerer Bruder & (0050) \\
\hline húg & (húga)/húgaim & ,jüngere Schwester' & (0088) \\
\hline
\end{tabular}

Tabelle 19: Im Korpus belegte Verwandtschaftstermini.

Zu diesen Person Person Relationen kommt eine weitere für die ob-ugrischen und viele anderen Sprachen typische Verwendung von relationalen Nomina im weiteren Sinne hinzu: So trifft beispielsweise der mansische Hauptheld $E k^{w} a$ Pifris ${ }^{j}$ in seinen Handlungen oft auf Personen, die dann im weiteren Verlauf als »Tante« oder "Onkel« bezeichnet werden, er hingegen wird als »Neffe» angesprochen - hier wird nicht das tatsächliche Verwandtschaftsverhältnis thematisiert, sondern das Gegenüber mit einem Respekt und Ansehen des Gegenüber implizierenden Terminus angesprochen.

(44) NM_text_012_0192:1

$\begin{array}{llllllll}\text { api } & - \text { ris }^{j} & - \text { uw } & a k^{\mathrm{w}} & -e & \text { pa:l } & \text { joxt } & -i \\ \text { nephew } & -\mathrm{DIM} & -\mathrm{SG}<1 \mathrm{PL} & \text { aunt } & -\mathrm{SG}<3 \mathrm{SG} & \text { to } & \text { arrive } & -\mathrm{PRS}[3 \mathrm{SG}]\end{array}$

'Our little nephew, as he arrives to his aunt.'

Der ausschlaggebende Faktor ist somit nicht das in der Terminologie suggerierte Verwandtschaftsverhältnis an sich, sondern die Interaktion zweier belebter Referenten. Die Relation Person Person scheint nach den Korpusbelegen die häufigste in der Konstruktion ausgedrückte Relation zu sein (905 Belege), eine Untergruppe bildet hier außerdem die Relation von Personen und Lebewesen im Allgemeinen (Person Lebewesen bzw. Lebewesen Person, zusammen 128 Belege) sowie zwischen einer Einzelperson und einer ganzen Gruppe von Personen (PERSON GRUPPE, 19 Belege). Die 68 Korpusbelege, in welchen der Kopf ein Eigenname ist, entsprechen bis auf eine Ausnahme ebenfalls dieser Relation. Das Beispiel zur "ad-hoc relation" bei Kenesei/Vago/Fenyvesi (Hg.) 1998 wäre in der vorliegenden Arbeit auch in dieser 
Kategorie einzuordnen. Dass es sich hierbei um eine Relation handelt, welche sich insbesondere im Handlungsverlauf zwischen zwei Referenten ergibt, bestätigt nicht nur die besondere Verwendung der Verwandtschaftstermini, sondern auch die Verteilung der Realisierung des Referenzpunktes: Dieser ist nahezu im allen Belegen mit einer Leerstelle realisiert (816 von 905 Belegen), was einer hohen Topikalität und Vorerwähntheit des Referenzpunktes entspricht. Es geht demnach bei der Erstellung der Relation zweier Personen weniger um den Ausdruck eines bestimmten verwandtschaftlichen Verhältnisses, sondern um den Ausdruck einer auf der Handlung bzw. der Situation basierenden Relation zwischen Partizipanten im Text bzw. den Sprechaktteilnehmern. Dies wird in Kapitel 5.2 unter Berücksichtigung der gesamten Textumgebung weiter spezifiziert werden. Die zweithäufigste Realisierung ist die mit einem Personalpronomen, dann erst folgen die wenigen Belege mit Substantiven als Referenzpunkt. Die Relation Person Person weicht damit von der Gesamtverteilung ab, was ebenfalls für eine Hervorstellung der Kategorie Person gegenüber den anderen Kategorien spricht.

\section{Ganzes Teil}

In dieser Gruppe sind alle Arten von Teil-Ganzes-Beziehungen zusammengefasst, auch die sogenannten erweiterten Relationen wie ,Spitze eines Berges' oder ,Ast eines Baumes' etc. (verglichen Kapitel 1.2).

(45) HU 0846

a másik oldal -á -n

the other side $-S G<3 S G$-SUP

'Auf der anderen Seite.'

Die Benennung weicht von der gewöhnlich gebrauchten ab (TeIL-Ganzes vs. Ganzes Teil), um in allen Benennungen die Reihenfolge der in Relation stehenden Referenten beizubehalten. Einige Ganzes TeIL-Verhältnisse werden auch hier wie erwähnt gesondert als Untergruppe geführt: PERson KöRPERTEIL sowie LEBEWESEN KÖRPERTEIL, eine dritte Untergruppe wird als GRUPPE EINER bezeichnet. Hierunter fallen nahezu alle Belege, in welchen das Possessivsuffix aus einer Gruppe von Referenten denjenigen markiert, der im Folgesatz allein weitergeführt wird (siehe dazu Kapitel 5.2.1.3 Known Group). Insgesamt werden 677 Belege zur Relation Ganzes TeiL gezählt, womit sie die zweitgrößte Gruppe der Vorkommen ausmacht (366 Belege davon entsprechen Relationen Person Körperteil, 34 Lebewesen Körperteil und 45 Gruppe Einer). Zwischen den Ganzes Teil-Relationen und dessen Untergruppen mit belebten Referenten unterscheidet sich die Relation in der Realisierung des Referenzpunktes: Während die Relationen PERSON KÖRPERTEIL den Realisierungen der Referenzpunkte der Verteilung im Allgemeinen entsprechen (die größte Gruppe bilden die Leerstellen, dann folgen die Substantive und zum Schluss die Pronomen), bildet die Realisierung als Substantiv die größte Gruppe bei den unbelebten Ganzes TeIL-Relationen, gefolgt von den Leerstellen und zum Schluss den Realisierungen mit Pronomen. Dabei treten nur im Ob-Ugrischen Personalpronomen auf, im Ungarischen handelt es sich ausschließlich um Relativ- oder Demonstrativpronomen. Dies korreliert mit der kodierten Person: Fast alle Belege der Konstruktion Pron Subs ${ }^{\text {Ganzes } \sim \text { Terl }}$-Px kodieren die dritte Person Singular im Einbesitz. In Relation zu belebten Referenten (PERSON KÖR PERTEIL) 
hingegen werden immerhin 57 mal erste Personen und 26 mal die zweiten Personen kodiert. Von den 283 kodierten dritten Personen entfallen 235 auf die dritte Person Singular im Einbesitz. Die Relation LeBEWESEN KöR PERTEIL wird in etwa zu gleichen Teilen mit einer Leerstelle oder einem Substantiv als Referenzpunkt realisiert. Pronomina kommen überhaupt nicht vor, was belegen könnte, dass Personalpronomina tatsächlich nur Personen und nicht allen belebten Referenten vorbehalten sind.

\section{Person Gegenstand}

In diese Gruppe fallen diejenigen Relationen, die im Allgemeinen als Besitzverhältnisse bezeichnet werden.

(46) SK_text_003_0221:2

\begin{tabular}{|c|c|c|c|}
\hline :moton & $\begin{array}{l}-\partial \phi \\
-S G<3 S G\end{array}$ & $\begin{array}{l}t^{i} \partial \\
\text { EMPH1 }\end{array}$ & $\begin{array}{l}\beta ə j \\
\text { take[PST] }\end{array}$ \\
\hline
\end{tabular}

'She took her suitcase with her.'

Hierunter werden sämtliche Relationen zwischen einer Person und unbelebten Gegenständen zusammengefasst. Es wird nicht unterschieden, ob es sich dabei um ein tatsächliches Eigentumsverhältnis handelt, da auch hier die sozio-kulturellen Ansichten variieren und die Verfasserin keinen Anspruch auf eine adäquate sozio-kulturelle Beschreibung von Besitzverhältnissen in ugrischen Sprachen erheben will. Insgesamt wurden 277 Belege mit dieser Relation festgestellt. Die Realisierung des Referenzpunktes entspricht in etwa der allgemeinen Verteilung, wobei Pronomen und Substantive in fast derselben Anzahl vorkommen.

\section{Person Lokalisation}

Diese Gruppe beinhaltet Relationen zwischen Personen und Ortsbezeichnungen, die Relation ergibt sich durch das Vorhandensein des Referenten am Ort oder seiner Bewegung dorthin bzw. von dort weg.

(47) NM_text_001_0088

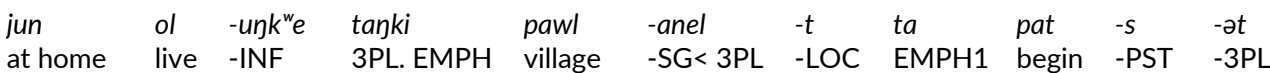

'So begannen sie in ihrem Heimatdorf zu leben.'

Es geht demnach wieder um eine situationsbedingte Verknüpfung. Auch Begriffe wie ,Haus' oder ,Bett', welche nach der üblichen Kategorisierung von Possessivkonstruktionen zum konkreten Eigentum gezählt werden würden, werden unter bestimmten Voraussetzungen, welche sich aus der konkreten Äußerung ableiten, als Lokalisation eingeordnet. Diese Gruppe machen 164 Belege aus, die Verteilung der Realisierung des Referenzpunktes entspricht in etwa der allgemeinen Verteilung, die Anzahl der Pronomina ist etwas höher als die der Substantive.

\section{Person Abstrakt}

In diese Gruppe fallen Relationen zwischen einem belebten Referenzpunkt (Person) und Abstrakta wie Maße/Zeitangaben, Gefühle und auch Bezeichnungen, welche synonym für die Person stehen, wie ,Seele' oder ,Herz. Ebenfalls eine Unterkategorie bilden Eigenschaften und Qualifizierungen, so entspricht die Kategorie AвsTraKT auch beispielsweise der Relation »property« von Kenesei/Vago/Fenyvesi (Hg.) 1998. 
(48) KK_text_008_0134

$\begin{array}{llll}\text { jer } & - \text {-amən } & \text { ijksm } & -ə t \\ \text { power } & -\mathrm{PL}<1 \mathrm{DU} & \text { equal } & -\mathrm{PL}\end{array}$

'Unsere Kräfte sind gleich.'

Die Gruppe beläuft sich letztlich auf 157 Belege, die Realisierung des Referenzpunktes entspricht der allgemeinen Verteilung.

\section{Person Resultat}

In dieser Gruppe werden Relationen zusammengefasst, welche einzig und allein durch die Handlung, die vom Referenzpunkt ausgeführt wird, Bestand haben. Während relationale Nomina zwar einen möglichen Referenzpunkt implizieren (verglichen Barker 2008), sind es doch Autosemantika, welche konkrete Objekte in der außersprachlichen Welt benennen. Dies trifft nicht auf Abstrakta wie ,Gesang', ,Gedanke` etc. zu, welche nur infolge der Handlung durch einen Agens bestehen.

(49) KK_satz_0010

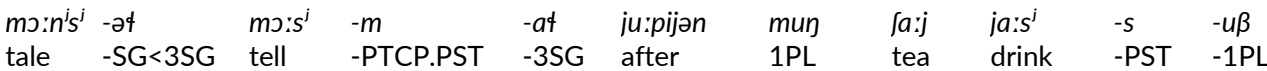

'Nachdem er sein Märchen erzählt hatte, tranken wir Tee.'

In den ugrischen Sprachen entsprechen solche Abstrakta häufig Nominalisierungen von Verbalhandlungen, gebildet mithilfe von Ableitungssuffixen, zum Beispiel Ungarisch -Ás. Sie machen generell den Großteil der Relationen, in welchen das Target dem Begriff Resultat entspricht, aus. Die von Kenesei/Vago/Fenyvesi (Hg.) 1998 als »action nominalization« bezeichneten Relationen würden dieser Kategorisierung entsprechen. Es sind insgesamt 116 Belege, die Realisierung des Referenzpunktes entspricht der Relation Person Gegenstand und weicht damit nur minimal von der allgemeinen Verteilung ab.

In geringerem Umfang sind auch andere Kombinationen im Korpus belegt, zum Beispiel Gegenstand Eigenschaft oder A bstra KT A AstraKt, welche nicht weiter analysiert wurden.

(50) HU 0197

az oktatás minőség -é -nek

the education quality $-S G<3 S G \quad-D A T$

'Die Qualität der Ausbildung.'

Erwähnt werden muss jedoch noch, dass Relationen, welche Abstrakta, jedoch keine Personen beinhalten, fast ausschließlich für das Ungarische und hier in der Mehrzahl (65 von 89 Belegen) in den Sachtexten enthalten sind.

Dies könnte für eine Grammatikalisierung zur Izafet-Konstruktion sprechen, wodurch die Zugehörigkeit von Kopf und Modifizierer grammatisch markiert wird und weniger Referenz aufgebaut wird. Eine Relation in der Konstruktion wäre in der Konsequenz nicht ohne Weiteres zu bestimmen bzw. nicht mehr vorhanden (siehe dazu auch Kapitel 5.2.2.1). Auch auf Konstruktionen mit Jahreszahlen im Ungarischen kann aufgrund fehlender Belege nicht weiter eingegangen werden.

Der Konstruktionstyp SuBs-Px entspricht der häufigsten Verwendung der Possessivsuffixe. Die häufigste Realisierung des Referenzpunktes ist die Leerstelle, die häufigste Relation geht von einem belebten Referenten aus. Beides spricht für die 
hohe Topikalität des im Possessivsuffix kodierten Referenten. Die am häufigsten kodierte Person ist die der dritten Singular im Einbesitz (siehe zum Beispiel Kapitel 6). Diese kommt sowohl am Häufigsten in den Relationen mit einem belebten Referenzpunkt als auch den GANZES TEIL-Relationen vor. In dieser Person ergibt sich damit die bedeutendste Distribution in der Verwendung der Possessivkonstruktion. Dies wird sich im folgenden Kapitel 5.2 in Zusammenhang mit der Textumgebung der einzelnen Konstruktionen noch verdeutlichen und zu einer möglichen Unterteilung des Possessivsuffixes der dritten Person Singular Einbesitz in zweierlei Kategorien von Suffix führen. Zuvor sollen noch die beiden anderen Konstruktionstypen analysiert werden. Die Terminologie der Referenzpunkt-Konstruktion wird auch hier beibehalten, sodass weiterhin der im Possessivsuffix kodierte Referent als »Referenzpunkt« bezeichnet wird. Von der Bezeichnung »Target « für den Kopf der Konstruktion wird abgesehen, um den Unterschied in der Anzahl der im Possessivsuffix kodierten Referenten zu verdeutlichen. Stattdessen wird vom »Kopf « der Konstruktion gesprochen.

\subsubsection{Die Konstruktion Verbalnomen-Possessivsuffix (VN-Px)}

Der folgende Abschnitt widmet sich den Konstruktionen mit einem Verbalnomen als Kopf. Wie in Kapitel 1.2 definiert, werden unter dem Begriff »Verbalnomen« sämtliche sogenannten infiniten Verbalformen der ugrischen Sprachen zusammengefasst. Art der Verbalnomina und auch welche Verbalnomina Possessivsuffixe tragen können, ist einzelsprachlich verschieden, weswegen auf jede der drei ugrischen Sprachen gesondert eingegangen werden muss. Zu den Verbalnomina in den ugrischen Sprachen zählen Partizipien, Konverben und Infinitive. Prinzipiell besteht die Konstruktion aus denselben Bestandteilen wie die Konstruktion Subs-Px, und der Modifizierer kann auf dieselbe Art realisiert werden:

\begin{tabular}{|c|c|c|c|c|c|}
\hline Typ & Modifizierer & & Kopf & & Vorkommen \\
\hline 2.b.1 & Substantiv & & Verbalnomen & $-P x$ & Mansisch, Chantisch, Ungarisch \\
\hline 2.b.2 & Pronomen & & Verbalnomen & $-P x$ & Mansisch, Chantisch \\
\hline 2.b.3 & $\varnothing$ & & Verbalnomen & $-P x$ & Mansisch, Chantisch \\
\hline 2.c.1 & Substantiv & $-C_{x}$ & Verbalnomen & $-P x$ & Ungarisch \\
\hline 2.c. 2 & Pronomen & $-C x$ & Verbalnomen & $-P x$ & Ungarisch \\
\hline
\end{tabular}

Tabelle 20: Realisierungen des Modifizierers in der Konstruktion VN-Px im Korpus der vorliegenden Arbeit.

Die Konstruktion VN-Px kann des Weiteren ebenfalls mit einem Determinierer in Form des Demonstrativpronomens erweitert werden. Dessen Rolle wird im Folgekapitel eingehender betrachtet (siehe Kapitel 5.2). Im Unterschied zur Konstruktion Subs-Px wird allerdings nur der Referenzpunkt im Possessivsuffix kodiert. 


\subsubsection{Infinitiv}

Der Infinitiv wird als »Nominalform des Verbs« (Bußmann 2002: 304) definiert, eine Infinitivkonstruktion ist eine satzwertige Konstruktion, da sie als Subjekt, Objekt, Prädikat, oder Attribut fungieren kann (verglichen Bußmann 2002: 304). Für die ugrischen Sprachen ist diese Definition jedoch nicht ausreichend, da sie auf alle Verbalnomina zutreffen kann.

Die Definition des Infinitivs in den ugrischen Sprachen wird zudem erschwert, da die Grenzen zwischen Partizip und Infinitiv nicht immer klar bestimmbar sind. Der Infinitiv auf - $t i$ im Kazym-Chantischen ist beispielsweise formgleich mit dem Partizip Präsens. Das Ungarische hingegen verfügt über eine gesonderte Infinitivendung - ni, dafür ist das Suffix des Partizip Perfekt - Vtt formgleich mit dem Vergangenheitsmarker am finiten Verb (und hat vermutlich denselben Ursprung). Des Weiteren werden Infinitive in der ugrischen Lexikografie nicht auf dieselbe Weise behandelt wie zum Beispiel in indo-europäischen Traditionen: Die Form des Verbs, welche für den Wörterbucheintrag im Ungarischen herangezogen wird, ist nicht die des Infinitivs, sondern der dritten Person Singular Präsens in der subjektiven Konjugation. Analog dazu werden die Verben in ob-ugrischen Wörterbüchern verzeichnet. Als Infinitive werden in der vorliegenden Arbeit Verbalnomina mit folgenden Suffixen bezeichnet:

$\begin{array}{ll}\text { Mansisch: } & -\eta k^{w} e \\ \text { Surgut-Chantisch: } & -t e\left(\gamma^{2}\right) \\ \text { Kazym-Chantisch: } & -t i \\ \text { Ungarisch: } & -n i\end{array}$

Die hier als Infinitive klassifizierten Suffixe nehmen in den ob-ugrischen Sprachen keine Possessivsuffixe $a^{97}$, im Ungarischen hingegen kann auch der Infinitiv in bestimmten Konstruktionen ein Possessivsuffix tragen. So kann auch hier die universale Definition des Infinitivs nicht ohne Weiteres auf den Ungarischen Infinitiv abgeleitet werden. Die Konstruktionen, in welchen der Infinitiv im Ungarischen ein Possessivsuffix trägt, sind sogenannte Modalverb-Konstruktionen. Diese Konstruktionen bestehen aus einem Modalverb und dem Infinitiv. Am Modalverb (dem Hilfsverb der Konstruktion) wird das Tempus kodiert, an der Infinitivform, welche dem Vollverb entspricht, die Person (Kenesei/Vago/Fenyvesi (Hg.) 1998: 318):

\begin{tabular}{lll}
\hline Adverbiale Bestimmung & Kopulaverb & Subjekt \\
\hline A korábbi EMT-tagok-nak & kellett & is pályáz-ni-uk \\
nek-em & nem lehet & megházasod-n-om \\
$\varnothing$ & kell-ett & men-ni-e \\
\hline
\end{tabular}

Tabelle 21: Struktur der Modalverb-Konstruktionen des Ungarischen.

97 Für das Mansische sind ebenfalls wenige Konstruktionen des Infinitivs mit Possessivsuffix belegbar. Diese sind jedoch archaisch (Murphy 1968: 128) und kommen außerdem nicht im Korpus vor. 
Die Verwendung des Possessivsuffixes kann als darin begründet angesehen werden, dass die Infinitivform die Rolle des Subjekts in dieser Konstruktion übernimmt.

Der am Infinitiv kodierte Referenzpunkt kann pronominal oder lexikalisch in der Konstruktion erwähnt werden, muss es jedoch nicht. In diesem Fall erhält er ebenfalls das dependent-marking mit dem Dativ (das Vorkommen des Konstruktionstyps 2.c.1 und 2.c.2 entfällt ausschließlich auf die Modalverb-Konstruktion) (Kenesei/Vago/Fenyvesi (Hg.) 1998: 318). Ähnlich wie bei der Habeo-Konstruktion ist die Suffigierung mit Possessivsuffix im Ungarischen meines Erachtens konstruktionsbedingt und obligatorisch. Sie sind entsprechend im Korpus markiert, werden jedoch nicht weiter analysiert.

\subsubsection{Partizip}

Partizipien werden ebenfalls oft zur Gruppe der Infinitive mit satzwertigen Funktionen gezählt (Bußmann 2002: 304). Die meisten Sprachen verfügen über mehrere Partizipialformen, die häufigsten sind wohl Partizip Präsens und Partizip Perfekt. Neben Tempus können Partizipien auch das Genus Verbi ausdrücken und - mithilfe der Possessivsuffixe - auch nach Person flektiert werden, sodass die Definition der unkonjugierten Verbformen so ebenfalls nicht zutrifft (verglichen Kapitel 1). Für die ugrischen Sprachen ist für die Partizipien außerdem charakteristisch, dass sie zwei Hauptfunktionen in der Satzbildung übernehmen: Die der attributiven Nebensätze (Relativsätze) und der subordinierenden Nebensätze (verglichen Nikolaeva 1999a: 47). Für die vorliegende Arbeit von Relevanz ist dabei die Possessivsuffigierung in beiden Konstruktionen: Erstere wurden im Abschnitt zur Konstruktion SuBS-Px thematisiert, da das Possessivsuffix nicht dem Partizip, sondern dem Substantiv angefügt wird. In den subordinierenden Nebensatzkonstruktionen befindet sich das Possessivsuffix am Partizip, welches somit den Kopf der Konstruktion bildet und daher in diesem Abschnitt behandelt wird.

Die Nebensatzkonstruktionen mit Partizipien können temporale, kausale, konditionale oder sonstige modale Relationen zum Hauptsatz ausdrücken. Sie werden vor allem in den ob-ugrischen Sprachen anstelle von koordinierenden Nebensätzen mit Konjunktionen verwendet. Das Partizip fungiert als Prädikat des Nebensatzes, die temporale oder adverbiale Bestimmung wird durch Postpositionen oder Lokalkasussuffixe, welche dem Partizip angefügt werden, ausgedrückt. Neben diesen Nebensatzkonstruktionen, welche im Hauptsatz die Rolle einer adverbialen Bestimmung einnehmen, sind auch einige Belege der Konstruktion in Form eines Objektsatzes vorhanden. Der Partizipialsatz enthält syntaktische Kernrollen und fakultative Ergänzungen je nach Satzaussage bzw. Prädikat, diese können koreferent mit Partizipanten des Hauptsatzes sein. Das Possessivsuffix kodiert das Subjekt des Nebensatzes an der Verbform des Nebensatzes.

Beide in der vorliegenden Arbeit berücksichtigten Dialekte des Chantischen verfügen über jeweils ein Partizip Präsens auf - $t$ (Kazym-Chantisch - $t i$ ) und ein Partizip Perfekt auf $-m$. Das Surgut-Chantische verfügt außerdem über ein Negations- und ein Konditionalpartizip (Schön 2014: 139), welche jedoch nicht im Korpus der vorliegenden Arbeit vorkommen. Das Mansische verfügt über zwei aktivische Partizipialformen (Partizip Präsens auf -ne und Partizip Perfekt auf $-m$ ) und über ein Partizip 
Passiv -im(a). Alle drei Formen sind im Korpus belegt. Das Negationspartizip -tal des Mansischen kommt hingegen nicht als Kopf der Konstruktion im Korpus der vorliegenden Arbeit vor. Die Partizipialformen des Ungarischen fungieren nur selten als Adverbialbestimmung. Konstruktionen mit dem Partizip Perfekt -Ot $(t)$ und Personalmarkierung in einer der Nebensatzkonstruktion ähnlichen Verwendung werden in ungarischen Grammatiken unter anderem als »Verbalnomen-Verba« bezeichnet (Keszler/ Lengyel 2008: 91). Im Hinblick auf die im Nebensatz beschriebene Handlung kommen kausale sowie temporale Nebensatzkonstruktionen im Korpus der vorliegenden Arbeit vor, in etwa gleichmäßig verteilt auf Gleichzeitigkeit und Vorzeitigkeit, am seltensten wird Nachzeitigkeit ausgedrückt.

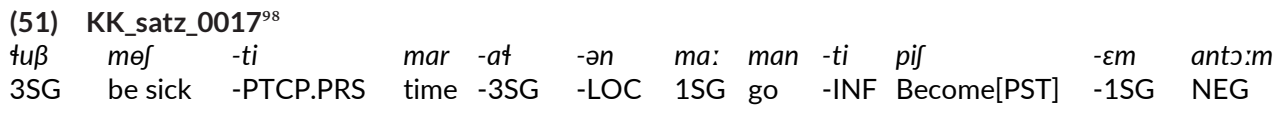

'Solange er krank ist, kann ich nicht kommen.'

Die Verwendung des Possessivsuffixes in den Nebensatzkonstruktionen wird ebenfalls in Zusammenhang mit der Topikalität des jeweiligen Referenten, welcher als Subjekt des Nebensatzes fungiert, gebracht. Bei Koreferenz mit dem Subjekt des Hauptsatzes beispielsweise muss die Partizipialform ein Possessivsuffix tragen (Nikolaeva/Kovgan/ Koškarëva 1993: 146), was nichts anderes bedeutet, als dass der Referenzpunkt sowohl in Haupt- als auch Nebensatz lediglich mit einer Leerstelle realisiert wird, was wiederum für einen Referenten mit hoher topikaler Rolle wie beispielsweise dem DiskursTopik spricht. Dies entspricht der Verteilung von pronominaler, lexikalischer und Erwähnung als Leerstelle in den Korpusbelegen: Letztere bildet die mit Abstand größte Gruppe der Partizipialkonstruktionen in beiden ob-ugrischen Sprachen.

Es ist jedoch zu erwähnen, dass die Verteilung der Partizipialkonstruktionen auf die drei ob-ugrischen Sprachen bzw. Dialekte unterschiedlich ist. Das Surgut-Chantische weist mit insgesamt 74 Belegen die meisten Partizipialkonstruktionen auf, nur zwölf davon werden nicht mit einer Leerstelle für den Referenzpunkt realisiert. Die anderen beiden Realisierungen verteilen sich gleichmäßig, sechsmal wird der Referenzpunkt als Substantiv realisiert, sechsmal mit Personalpronomen. Die Verteilung im KazymChantischen ist ähnlich, die Realisierungen von Substantiv oder Pronomen als Referenzpunkt sind beinahe gleich verteilt, jedoch ist der Anteil an Leerstellen mit $27 \mathrm{Be}$ legen etwas geringer in der Relation als im Surgut-Chantischen. Die wenigsten Belege für diese Konstruktion weist das Mansische mit 30 Vorkommen auf. Doch auch hier machen die Konstruktionen mit Leerstelle etwa zwei Drittel der Belege aus. Lediglich die Belege mit Pronomen (neun) übersteigen die mit Substantiv (zwei). Für das Ungarische lassen sich zwei Belege aus dem Korpus den Nebensatzkonstruktionen zuordnen, beide mit Substantiven als Referenzpunkt und beide fungieren als Objektsatz. Die Vorkommen entsprechen insgesamt der Korrelation von Topikalität und anaphorischem Verweismittel (siehe Kapitel 3), und die Verteilung der Leerstelle gegenüber den anderen Realisierungen entspricht in etwa der der Konstruktion Subs-Px, was zum einen

98 In dieser Konstruktion erscheint das Possessivsuffix, welches den Referenzpunkt am Verbalnomen kodiert, an der Postposition, da diese die äußerste rechte Position in der Konstruktion einnimmt. Siehe hierzu und zur Postposition mar Kapitel 5.1.3. 
für die Kodierung topikaler Referenzpunkte und zum anderen für die Default-Verwendung der Leerstelle für diese spricht. Die Relation zwischen Substantiv und Pronomen als Realisierung des Referenzpunktes scheint jedoch leicht gegenüber der Konstruktion Subs-Px abzuweichen. Weitere Aussagen lassen sich zunächst aufgrund der geringen Datenmenge nicht treffen.

Zwei weitere Partizipialkonstruktionen des Surgut-Chantischen fungieren in der Form einer Negationskonstruktion. Das an sich finite Prädikat wird mithilfe einer Partizipialkonstruktion mit Possessivsuffix ausgedrückt, welcher eine Negationspartikel folgt.

(52) SK_text_003_0006:3

\begin{tabular}{|c|c|c|c|c|c|c|c|}
\hline $\begin{array}{l}\text { əj } \downarrow \text { \&b:tnə } \\
\text { once }\end{array}$ & $\begin{array}{l}\text { əj } \\
\text { one }\end{array}$ & $\begin{array}{l}\text { i:mi } \\
\text { old woman }\end{array}$ & $\begin{array}{l}-4 \\
-S G<3 S G\end{array}$ & $\begin{array}{l}4 H \beta \\
3 S G\end{array}$ & $\begin{array}{l}\text { quit } \\
\text { house }\end{array}$ & $\begin{array}{l}-2 \phi \\
-S G<3 S G\end{array}$ & $\begin{array}{l}-D \\
- \text { DLAT }\end{array}$ \\
\hline $\begin{array}{l}\text { 40:yəttə } \\
\text { visit }\end{array}$ & $\begin{array}{l}-m \\
- \text { PTCP.PST }\end{array}$ & $\begin{array}{l}-04 \\
-3 S G\end{array}$ & $\begin{array}{l}\text { antem } \\
\text { does no }\end{array}$ & & & & \\
\hline
\end{tabular}

'Once one of the women does not go to the house of the other.'

Weiteres über die Partizipialkonstruktion an sich und die Verwendung des Possessivsuffixes lässt sich aufgrund der wenigen Belege nicht aussagen, weswegen diese Belege keine weitere Berücksichtigung in der Analyse finden.

Das Nomen Actionis wird in der vorliegenden Arbeit aus drei Gründen nicht in diesem Konstruktionstyp berücksichtigt: Erstens sind keine Vorkommen im Korpus für das Chantische belegt. Zudem ist die Bestimmung im Mansischen nicht ohne Weiteres möglich, da das Nomen Actionis hier formgleich mit dem Partizip Perfekt ist und eine Analyse zur konkreten Unterscheidung bislang fehlt. Zuletzt kann nur für das Ungarische ein deverbales Ableitungssuffix belegt werden, welches nach der Definition des Nomen Actionis als »von Verben abgeleitete Substantive, die sich auf Handlungen und Vorgänge beziehen« (Bußmann 2002: 469), gelten kann. Im HNC sind Formen mit dem Ableitungssuffix -Ás als Substantive getaggt, und auch im Wörterbuch erhalten solche Wortformen einen eigenen Eintrag als Lemma. Wortformen mit dem Ableitungssuffix -Ás (glossiert als NMLZ) werden in der vorliegenden Arbeit gemäß ihrer Definition unter der Konstruktion SuBs-Px behandelt und dort jedoch gesondert vermerkt, sodass das Nomen Actionis insgesamt keine Berücksichtigung im Konstruktionstyp VN-Px erfährt.

Ähnlich wie bei Konstruktionen mit Modalverb und Infinitiv im Ungarischen gibt es in den ob-ugrischen Sprachen Konstruktionen mit Partizipien, welche ebenfalls konstruktionsbedingt mit einem Possessivsuffix auftreten, und zwar im Evidentialis oder Mirativ.

(53) SK_text_001_0008:2

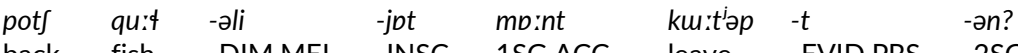

back fish -DIM.MEL -INSC 1SG.ACC leave -EVID.PRS -2SG

'Hast du mir den Rücken des Fischleins (übrig) gelassen?'

(54) NM_text_004_0080

\begin{tabular}{|c|c|c|c|c|c|c|}
\hline $\begin{array}{l}\text { nasa: } t^{\prime} i \\
\text { as it turns out }\end{array}$ & $\begin{array}{l}s^{j} a: n^{j} \\
\text { mother }\end{array}$ & $\begin{array}{l}-e \\
-S G<3 S G\end{array}$ & $\begin{array}{l}\text { wo:rut } \\
\text { forest spirit }\end{array}$ & $\begin{array}{l}\text { o:jka } \\
\text { old man }\end{array}$ & $\begin{array}{l}o: s^{j} \\
\text { have }\end{array}$ & $\begin{array}{l}-n e \\
-M I R . P R S\end{array}$ \\
\hline
\end{tabular}

'Wie sich herausstellte, ist seine Mutter mit einem Waldgeist verheiratet.'

Als Mirativ wird eine »linguistische Markierung, dass eine Aussage eine Information enthält, die für den Sprecher in irgendeiner Art unerwartet ist « (Skribnik 2012: 3), bezeichnet. 
Sie stellt damit eine Weiterentwicklung der grammatischen Kategorie Evidentialität dar. Evidentialität wird als »strukturelle Dimension der Grammatik, deren Werte von Konstruktionstypen ausgedrückt werden, die die Quelle der Information kodieren, die ein Sprecher weitergibt«, definiert (Bußmann 2002: 206). Die Quelle der Information ist demnach wichtig, die Kategorie Evidentialität drückt verschiedene semantische Aspekte aus, die von Sprache zu Sprache verschieden sind. Im Chantischen können diese zum Beispiel Hörensagen, Inferentialität und auch Mirativität sein (Nikolaeva 1999a: 89f.).

Während der Mirativ somit im Chantischen bislang eher als Unterart der Evidentialität angesehen wird, welcher insbesondere in Verbindung mit den ersten Personen ein überraschendes und unerwartetes Ereignis und somit einen Kontrollverlust impliziert (Nikolaeva, 1999b: 146), wird zumindest für das Mansische in neueren Forschungen eine andere, unabhängige Entwicklung des Mirativs außerhalb der Unterkategorie von Evidentialität angenommen, welche die Möglichkeit der Entwicklung einer eigenständigen linguistischen Kategorie in Betracht zieht, wobei entgegengesetzt zur Evidentialität eben keine Angaben zur Quelle einer Information erfolgen (Skribnik 2012: 4). Da somit die Forschung in diesem Bereich ebenfalls nicht abgeschlossen ist, kommen zur Verdeutlichung zwei Bezeichnungen zum Einsatz: "Mirativität" ist eine semantische Nuancierung von Evidentialität (zum Beispiel im Chantischen), der »Mirativ« ist eine eigenständige verbale Kategorie (zum Beispiel im Mansischen). Die inhärenten semantischen Aspekte der verbalen Kategorie Mirativ sind plötzliche Entdeckung und Erkenntnis bzw. Realisierung einer Tatsache, Überraschung und »unpreprared mind «, welche - anders als für Mirativität oft attestiert - nicht ausschließlich nur mit der ersten Person korrelieren. Der Mirativ kann auf Sprecher, Hörer und auf eine dritte Person in einer Erzählung zutreffen und nur in sekundärer Bedeutung auch evidentielle Aspekte ausdrücken (Skribnik 2012: 5-9). Eine detailliertere Analyse der Gemeinsamkeiten und Unterschiede soll nicht Gegenstand der vorliegenden Arbeit sein.

Gebildet werden mirative wie evidentielle Formen in beiden ob-ugrischen Sprachen mit Partizipialformen, welche mit Personalendungen versehen als finite Verbalformen fungieren. Je nach Tempus wird das Partizip Präsens oder Perfekt verwendet, im (Nord-) Chantischen ist auch eine Futur-Form und eine passive Form (hier entfällt die Personalmarkierung am Partizip Perfekt und nur der Numerus wird kodiert) der Evidentialität möglich, welche jedoch äußerst selten sind (Nikolaeva, 1999a: 88). Das Mansische bildet mit dem Partizip Passiv entsprechend passivische Formen. Zusätzlich können Adverbien wie mansisch nasati $i$,wie es sich herausstellt' den mirativen bzw. evidentiellen Charakter der Aussage unterstreichen. Die verwendeten Personalparadigmen unterscheiden sich je nach Tempus. Die Formen des Evidentialis und Mirativ Präsens werden mit dem Paradigma der Possessivsuffixe gebildet, in den Formen der Vergangenheit kommen sowohl das Paradigma der subjektiven als auch der objektiven Konjugationen zum Einsatz. Bei der Passivbildung im Mansischen ist nur das Paradigma der subjektiven Konjugation beteiligt. Die unterschiedliche Verwendung von Personalparadigmen resultiert vermutlich aus unterschiedlichen Grammatikalisierungspfaden.

Der Mirativ Präsens resultiert aus der Grammatikalisierung von Partizipialkonstruktionen, in denen das finite Prädikat weggefallen ist. Hier handelt es sich vor allem um Konstruktionen mit Verben der Wahrnehmung (Skribnik 2012: 20), zum Beispiel sujti ,zu hören sein' und nayki, zu sehen sein'. Diese Konstruktionen erhalten nominale 
Personalmarker am eingebetteten Partizipialsatz, da es sich um ursprünglich infinite Verbalformen handelt. Hier werden daher die Possessivsuffixe verwendet (Skribnik 2012: 21). Diese Konstruktionen sind ebenfalls noch produktiv, wie Belege aus dem Korpus zeigen:

(55) NM_text_014_0025

$\begin{array}{llllllll}\text { kol } & \text { sis } & -t & \text { potərt } & \text {-ane: } & -t & \text { sujt } & -i \\ \text { house } & \text { back } & \text {-LOC } & \text { tell } & \text {-PTCP.PRS } & -3 S G & \text { to be heard } & \text {-PRS[3SG] }\end{array}$

'Hinter dem Haus sind Stimmen zu hören.'

Dieser Grammatikalisierungspfad ist ebenfalls für den Mirativ Perfekt anzunehmen, auch hierzu findet sich ein Beleg im Korpus:

(56) NM_text_008_0100:1

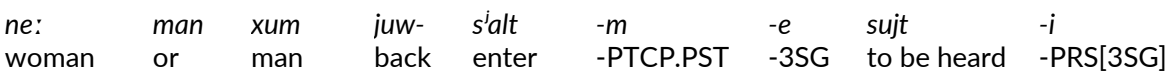

'A woman or a man can be heard entering the tent.'

Es ist also durchaus möglich, dass auch im Mirativ Perfekt das nominale Personalparadigma verwendet wird, welches fast formgleich mit dem der objektiven Konjugation ist. Da nicht ausreichend Belege vorhanden sind und die häufigste Personalendung, die dritte Person Singular, homonym ist, lässt sich dieser Punkt nicht abschließend klären. Einen möglichen Erklärungsansatz bietet Skribnik 2012. In der vorliegenden Arbeit wird weiterhin von der oben genannten Verteilung der Personalmarker ausgegangen. Eine zentrale Fragestellung für die vorliegende Arbeit bleibt jedoch, wie mit den Formen des Mirativs verfahren wird. Aufgrund des derzeitigen Forschungsstandes werden Formen des Mirativs im Mansischen sowie des Evidentialis im Chantischen als finite Verbalformen interpretiert. Aus diesem Grund ist die Markierung mittels Possessivsuffixen konstruktionsbedingt obligatorisch (als Personalmarker des Subjektes einer finiten Verbalform).

In den beschriebenen Konstruktionen mit Verben der Wahrnehmung im Mansischen scheint das Possessivsuffix ebenfalls eine konstruktionsbedingte obligatorische Markierung zu sein. Dies lässt sich anhand einer Analyse der kodierten Referenzpunkte belegen: Das Possessivsuffix erscheint nicht nur bei topikalen Referenten als Referenzpunkt, zum Beispiel E: $k^{w} a$ Pirris ${ }^{j}$ (NM_text_009_0090:5), sondern auffällig häufig handelt es sich beim lexikalisch erwähnten Referenzpunkt um ein Indefinitpronomen, sodass hier die Topikalität des Referenzpunktes offenbar kein Kriterium für die Markierung mit Possessivsuffix darstellt. Bei Beispiel (56) kann sogar von einer kataphorischen Verwendung des Possessivsuffixes ausgegangen werden, da der als Leerstelle realisierte Referenzpunkt erst im Folgesatz lexikalisch erwähnt wird, wohingegen die erste Erwähnung in Form des Possessivsuffixes in dieser Konstruktion erfolgt.

Des Weiteren findet sich im gesamten Korpus keine einzige Konstruktion vom hier beschriebenen Typ, in welcher kein Possessivsuffix erscheint. Dasselbe ist für das Verb $n a y k i$,zu sehen sein' anzunehmen. Auch im Chantischen scheint diese Konstruktion vorzukommen, mit dem Verb se:tjat, zu hören sein' im Surgut-Chantischen und sa: $t^{j}$ at ,id. 'im Kazym-Chantischen. Hier scheint es sich des Weiteren ebenfalls um eine konstruktionsbedingte Markierung mit dem Possessivsuffix zu handeln. Für beide Dialekte ist jeweils zwar nur ein Beleg vorhanden, in beiden wird der Referenzpunkt jedoch mit einem Indefinitpronomen realisiert, was den zuvor für das Mansische beschriebenen Beobachtungen entspricht. 


\subsubsection{Konverb}

Als Konverben bezeichnet man Verbalnomina, welche vornehmlich adverbial, das heißt zur Modifizierung des verbalen Prädikats verwendet werden (Murphy 1968: 124). Sie drücken häufig die Art und Weise einer Handlung aus und implizieren dabei Gleichzeitigkeit mit der im Prädikat ausgedrückten Handlung (Filchenko 2007: 174).

(57) NM_text_011_0008

$\begin{array}{lllllll}\text { ula } & -t e & \text { puwl } & \text {-ime } & -t e & \text { tus } & -e \\ \text { fire } & \text {-SG<3SG } & \text { blow } & \text {-CVB } & \text {-3SG } & \text { moustache } & \text {-SG<3SG } \\ \text { ulja } & -n & \text { non- } & \text { pelamt } & \text {-awe } & -s & \\ \text { fire } & \text {-DLAT } & \text { up } & \text { ignite } & \text {-PASS } & \text {-PST[3SG] }\end{array}$

'Während er das Feuer anfacht. wurde sein Bart von Feuer angezündet.'

Die Konverben -man des Kazym-Chantischen und -min des Surgut-Chantischen werden anders als die Konverben im Mansischen nicht mit Possessivsuffixen versehen (verglichen Filchenko 2007: 291). Die mansischen Konverben -im(a) und auch -ke kommen im Korpus der vorliegenden Arbeit vor, wobei -ke nur einmal belegt ist. Die restlichen neun Belege beinhalten das Konverb -im(a). Auch die ungarischen Formen - $v A$ und $-v A \hat{n}$ werden nicht mit Personalmarkern versehen (Keszler/Lengyel 2008: 95).

Hinsichtlich der Funktion des Konverbs, das Verb abverbial zu bestimmen, ist die Subjektidentität zwischen Konverb und Verb anzunehmen. Dies trifft jedoch nicht auf alle Belege im Korpus zu, wenngleich bis auf eine Ausnahme der Referenzpunkt mit einer Leerstelle in der Konverb-Konstruktion realisiert wird. In einer Konstruktion ist der Referenzpunkt trotz Subjektidentität als Substantiv realisiert, dieser Beleg (NM_ text_007_0001:5) befindet sich jedoch am Beginn einer Erzählung, sodass es durchaus denkbar wäre, dass die lexikalische Erwähnung eher Subjektposition des Hauptsatzes und nicht des Nebensatzes entspricht:

(58) NM_text_007_0001:5

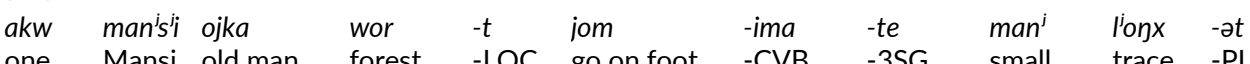
one Mansi old man forest -LOC go on foot -CVB -3SG small trace -PL

'Als ein alter mansischer Mann im Wald unterwegs war, erblickte er kleine Spuren.'

Die Koreferenz wird bei den Partizipialkonstruktionen als ausschlaggebender Faktor für die Possessivsuffigierung angesehen (siehe Nebensatzkonstruktionen). Somit müssen sowohl der Possessivsuffigierung der mansischen Konverben als auch der Partizipialkonstruktionen in allen ob-ugrischen Sprachen weitere Faktoren zugrunde liegen, welche im zweiten Teil des Kapitels mit Zuhilfenahme der Textumgebung genauer bestimmt werden sollen. Zuvor wird noch die Konstruktion PostP-Px vorgestellt.

\subsubsection{Die Konstruktion Postposition-Possessivsuffix (PostP-Px)}

Der dritte Konstruktionstyp, welcher in der vorliegenden Arbeit Berücksichtigung findet, ist der Konstruktionstyp PозтP-Px; das heißt, eine Postposition bildet den Kopf der Konstruktion. Wie eingangs erwähnt sind Postpositionen Adpositionen, welche dem Bezugswort folgen und mitunter aus Substantiven grammatikalisierte Synsemantika sind. Auch Postpositionen können mit Personalsuffixen versehen werden, ihrer Grammatikalisierung entsprechend tragen sie ebenfalls die nominalen Personalmarker, das heißt Possessivsuffixe. 
Im Ob-Ugrischen können Postpositionen des Weiteren mit Kasussuffixen versehen werden. Hierbei sind zunächst Postpositionen mit produktiven Kasussuffixen zu erwähnen, welche eine Lokalkasusreihe nach dem Muster Source-Path/Location-Goal bilden können (sogenannte reihenbildende Postpositionen, Schön 2014: 51). Die Reihenfolge von Kasus- und Possessivsuffix ist meistens PostP-Px-Cx (Murphy 1968: 54). Insbesondere im Chantischen können einige Postpositionen aber auch fossilisierte Formen von Kasussuffixen beinhalten, diese werden im Gegensatz zu den reihenbildenden Postpositionen nicht in Stamm und Kasussuffix segmentiert (verglichen Schön 2014: 83). ${ }^{99}$ Insbesondere in Kombination mit der Kategorie Person, das heißt in Verbindung mit Personalpronomina, werden Konstruktionen mit Postpositionen im Ob-Ugrischen der Kasus-Suffigierung des Pronomens vorgezogen (Kálmán 1965: 42), zum Beispiel am jotam ,mit mir' (NM 1828) statt a:nəmtal ,id..

Die Postpositionen im Ungarischen sind ebenfalls reihenbildend, zum Beispiel alatt ,unter ' - alá ,nach unten ' - alól, von unten. Auch wenn eine Segmentierung in Stamm und Suffix möglich scheint, werden die Kasussuffixe bei der Glossierung ebenfalls nicht vom Stamm der Postposition getrennt. Des Weiteren entsprechen die Suffigierungen der Postpositionen nicht unbedingt den jeweiligen Kasussuffixen der anderen nominalen Paradigmen und sind mitunter auch nicht mehr produktiv (zum Beispiel - $t t$, LOC').

Ebenfalls in Zusammenhang mit der Grammatikalisierung aus Substantiven steht die Frage, ob Postpositionen überhaupt den Kopf einer Konstruktion bilden können (verglichen Murphy 1968: 139). So kann einerseits mit dem Ursprung vieler Postpositionen in einem Substantiv dafür argumentiert werden, diese als Kopf der Konstruktion anzusehen. Außer Acht gelassen werden darf dabei jedoch nicht, dass Postpositionen in ihrer Funktion als meist lokal-adverbiale Bestimmung ebenfalls dem Bezugswort folgen und damit zwar die am weitesten rechte Position in der Konstruktion einnehmen, jedoch nicht zwangsläufig als Kopf fungieren. So ist es möglich, dass ein Possessivsuffix, welches mit einem Substantiv eine Konstruktion bildet, stattdessen der Postposition angefügt wird, welche das Substantiv lokal-adverbial bestimmt (verglichen Nikolaeva 1999a: 36).

\begin{tabular}{lll}
\hline Referenzpunkt & KOPF & \\
\hline PERSPR & SUBS & POSTP-PX \\
\hline ma: & xo:t & punt-Em -ən (KK 1102) \\
1SG & house & to 1SG LOC \\
\hline nan & xo:t-en & Benta (KK 1054) \\
2SG & house & until \\
\hline PERSPR & PoSTP-Px & \\
\hline ma: & xosia -m -a (KK 1099) & \\
1SG & at 1SG DLAT & \\
\hline
\end{tabular}

Tabelle 22: Struktur der Konstruktion PERSPR SUBS-Px mit Postposition gegenüber der Konstruktion PERSPR POST-PX.

99 Ein Abtrennen dieses im Stamm fossilisierten Kasussuffixes würde bei zusätzlicher Possessivsuffigierung dieser Postposition in der Reihenfolge Stamm -Cx-Px resultieren, auf welche zuvor verwiesen wurde. 
Aus diesem Grund müssen hier ähnlich wie bei den Konstruktionen Subs-Px zunächst die einzelnen Bestandteile der Konstruktion voneinander unterschieden werden. Im ersten Beleg ma: xo:t puyteman bilden Kopf und Postposition eine untrennbare Einheit (Schön 2014: 80), das Possessivsuffix an der Postposition kodiert den Referenzpunkt (PersPr) des Kopfes (Subs), eindeutig an der Kongruenz des Personalpronomens (1SG) und des Possessivsuffixes (1SG) zu erkennen. Es handelt sich um die Konstruktion Subs-Px sowie auch um den Beleg nay xo:ten Benta. Im Beleg am pa:ltum fungiert die Postposition als Kopf, das Possessivsuffix kodiert den Referenzpunkt der Postposition.

Der Referenzpunkt kann in der Konstruktion PosTP-Px ebenfalls in verschiedenen Realisierungen auftreten:

\begin{tabular}{lllll}
\hline Typ & Modifizierer & Kopf & Vorkommen \\
\hline 3.b.1 & Substantiv & Postposition & $-\mathrm{Px}$ & Mansisch, Kazym-Chantisch \\
\hline 3.b.2 & Pronomen & Postposition & $-\mathrm{Px}$ & Mansisch, Chantisch \\
\hline 3.b.3 & $\varnothing$ & Postposition & $-\mathrm{Px}$ & Mansisch, Chantisch, Ungarisch \\
\hline
\end{tabular}

Tabelle 23: Realisierungen des Modifizierers in der Konstruktion PostP-Px im Korpus der vorliegenden Arbeit.

Die Realisierung des Referenzpunktes als Substantiv kommt am seltensten im Korpus vor. Im Kazym-Chantischen ist nur ein einziger Beleg dieser Konstruktion zu finden, dieser hat mit fast allen Belegen des Mansischen vom Konstruktionstyp Su Bs PosTP-Px gemeinsam, dass die Postpositionen allesamt der Gruppe von Lemmata angehören, deren Wortart nicht ganz eindeutig als Postposition oder Substantiv zu bestimmen ist (verglichen Kapitel 4.2). Die Postposition tip ,innen' ist ebenfalls aus einem Substantiv grammatikalisiert (Schön 2014: 210) und damit vergleichbar mit den mansischen Vorkommen des Lemmas kiwre tij:wre ,Inneres'. Lediglich auf einen einzigen Beleg des Mansischen trifft diese Beschreibung nicht zu. Aufgrund der geringen Datenmenge lässt sich hierzu jedoch keine weitere Aussage treffen. Am zweithäufigsten kommen die Konstruktionen mit Personalpronomen als Referenzpunkt vor, allerdings findet sich kein einziger Beleg aus dem Ungarischen für diesen Konstruktionstyp. Die größte Gruppe bilden die Konstruktionen mit einem als Leerstelle realisierten Referenzpunkt, wohingegen nur ein Beleg aus dem Mansischen vertreten ist (2315). Dieser und auch die zwei Belege des Kazym-Chantischen $(1193,1199)$ gehören zu den Doppelformen Subs PostP (siehe zur Bestimmung der Wortart).

Damit weichen die Konstruktionen mit Postpositionen in mehreren Aspekten von den anderen beiden bislang beschriebenen Konstruktionen ab, und die Reihenfolge weicht in der Verteilung der Realisierung des Referenzpunktes gegenüber den Konstruktionen Subs-Px und VN-Px ab. Wurde bislang die Realisierung als Leerstelle als häufigste Konstruktionsart von der Realisierung als Substantiv gefolgt und wiesen die Belege mit Personalpronomen die geringste Anzahl auf, ist nun die Realisierung mit Personalpronomen die zweithäufigste Konstruktionsart, und Belege mit Substantiven als Referenzpunkt bilden die kleinste Gruppe. Des Weiteren erscheint - abgesehen 
vom dependent-marking - zum ersten Mal eine Distribution zwischen der Realisierung des Referenzpunktes und den Sprachen respektive Dialekten. Während das Fehlen von Belegen mit Pronomen und Leerstelle der Konstruktion VN-Px im Ungarischen ähnlich wie das Fehlen von Belegen mit Relativsatz der Konstruktion Subs-Px im Surgut-Chantischen möglicherweise noch durch die geringe Datenmenge erklärt werden kann, scheint die Verteilung der verschiedenen Realisierungen in der Konstruktion PostP-Px einem System zu folgen.

\subsubsection{Die Konstruktion PostP-Px im Ungarischen}

Das Fehlen des Konstruktionstyps PostP-Px mit Personalpronomen kann im Ungarischen möglicherweise mit einer Analogie zu den affigierten Formen der Personalpronomen in Zusammenhang gebracht werden. ${ }^{100}$ Die Bildung beider erfolgt strukturell ähnlich, der lokal-adverbialen Bestimmung in Form eines Kasussuffixes oder einer Postposition folgt eine Personalendung, welche in beiden Fällen auf die Possessivsuffixe zurückgehen:

$\begin{array}{lll}\text { mellett -e } & \text { next to -3SG } & \text { 'neben ihm/ihr' } \\ \text { nek -em } & \text { DAT -3SG } & \text {,mir' }\end{array}$

Die affigierten Formen der Personalpronomen können fakultativ mit dem entsprechenden Personalpronomen erweitert werden, dies kommt jedoch sehr selten vor, verglichen én töl -em ,1SG ABL -1SG' (HU_text_001_0118). Diese Erweiterung ist auch für die Postpositionen möglich, die Struktur ist auch hier dieselbe. Das Korpus der vorliegenden Arbeit weist keinen solchen Beleg auf, aber im HNC sind solche, wenn auch in sehr geringem Umfang, zu finden, zum Beispiel én mellett -em ,1SG neben-1SG (HNC_Abfrage_0044.pdf). ${ }^{101}$

Möglicherweise ist auch die Bildung der Personalpronomina analog zu der der Postpositionen erfolgt; eine strukturelle Ähnlichkeit ist jedenfalls nicht von der Hand $\mathrm{zu}$ weisen, sodass meines Erachtens für die Konstruktion PозтP-Px im Ungarischen eher ein Zusammenhang zu den affigierten Formen der Personalpronomina besteht als zur Possessivkonstruktion. Diese Annahme wird auch durch Reichert 1986 unterstützt, welcher die Konstruktion PоsтP-Px zusammen mit den affigierten Formen der

100 Das ungarische Personalpronomen wird außer im Akkusativ nicht mit Kasussuffixen dekliniert, sondern das jeweilige Kasussuffix oder eine davon abgeleitete Form fungiert als Art Stamm, dem ein Personalmarker angefügt wird, z.B. rá-nk,SUB-1PL', belöl-ed ,ELAT-2SG' (> Elativ bÓl). Die vorliegende Arbeit geht davon aus, dass dem Kasusmarker eine Leerstelle vorangeht, die unter bestimmten Umständen vom jeweiligen Personalpronomen im Nominativ aufgefüllt wird: 0 -vel-em, $\varnothing-\mathrm{COM}-1 \mathrm{SG} \mathrm{c}^{\circ}$ verglichen én vel-em ,1SG COM-1SG'. Die Formen des Akkusativs sind ebenfalls unregelmäßig, enthalten aber teilweise das Suffix des Akkusativ, z. B. ö- $t$,3SG-ACC.

101 Die betreffende Suchabfrage lieferte lediglich zwei Treffer. Allerdings weisen beide Ergebnisse weitere Formen im selben Satz auf. Eine gezieltere Suche nach den betreffenden Formen ist durch die Annotationsweise des HNC jedoch nicht möglich. 
Personalpronomen in einem Abschnitt behandelt (»Personalsuffixe an Kasuselementen/Postpositionen«, Reichert 1986: 71f.).

\subsubsection{Die Konstruktion PostP-Px im Ob-Ugrischen}

Im Mansischen und Kazym-Chantischen hingegen scheint die Realisierung des Referenzpunktes als Leerstelle beim Konstruktionstyp PosтP-Px nicht möglich zu sein. Dies ist den beiden anderen Konstruktionen Subs-Px und VN-Px völlig entgegengesetzt und entspricht nicht der bisherigen Beschreibung der Verwendung des Possessivsuffixes für das Ob-Ugrische. Hier muss der Verwendung des Possessivsuffixes demnach ebenfalls eine andere Motivation zugrunde liegen, für welche im folgenden Kapitel ein Erklärungsansatz vorgeschlagen wird. In diesem Zusammenhang muss noch auf die 29 Belege der Konstruktionsart PosтP-Px mit Leerstelle im Chantischen hingewiesen werden. Für das Chantische wird ebenfalls beschrieben, dass Postpositionen nicht ohne Bezugswort stehen können und auch den possessivsuffigierten Formen üblicherweise ein Personalpronomen vorangeht (Kovgan 2005: 555). Bei den 29 Belegen, welche dieser Beschreibung nicht entsprechen, handelt es sich in knapp zwei Drittel der Fälle (18 Belege) um die Postposition ont ,in' des Surgut-Chantischen. Diese Postposition wird beschrieben als eine der sehr seltenen Formen, welche ohne Bezugswort stehen kann (verglichen Schön 2014: 80). Dass die Form ausschließlich in der Kombination ontatna nomaqsat, er/sie sagt zu sich selbst' auftritt ${ }^{102}$, könnte außerdem dafür sprechen, dass es sich hier inzwischen um eine feste Phrase zur Einleitung eines Monologs in einer Erzählung handelt. Bei den restlichen Fällen des Surgut-Chantischen handelt es sich ebenfalls um Grammatikalisierungen von Substantiven (qu:tig ,zwischen' und pu:r ,hinter'; Schön 2014: 200 und 230), welche in der Konstruktionsart PosT-Px mit Leerstelle erscheinen.

Dies spricht meines Erachtens dafür, dass auch im chantischen die Postpositionen in der Regel nicht in der Konstruktionsart РозтP-Px mit Leerstelle vorkommen, da die wenigen Belege, welche dem nicht entsprechen, möglicherweise ebenfalls im $\mathrm{Zu}$ sammenhang mit den Doppelformen Suвs PostP gebracht werden können. Für das Kazym-Chantische ist aufgrund der begrenzten Anzahl der Belege keine weitere Aussage zu treffen, außer dass es sich bei den beiden Belegen mit Leerstelle (1193 und 1199) ebenfalls um die zuvor erwähnten Doppelformen Subs PostP tip ,innen' handelt. In den Doppelformen Subs PostP scheint somit die Realisierung des Referenzpunktes sowohl als Leerstelle als auch als Substantiv möglich, wohingegen in den Konstruktionen, welche wahrscheinlich ausschließlich zur Postposition gezählt werden können, nur das Personalpronomen als Realisierung des Referenzpunktes im Ob-Ugrischen infrage kommt.

Insgesamt ist festzustellen, dass die Konstruktion PostP-Px zum einen nicht ohne Weiteres von der Konstruktion Subs-Px hinsichtlich der Wortart abzugrenzen ist. Auf der anderen Seite jedoch lassen sich gewisse Regelmäßigkeiten erkennen. Vom Versuch einer - eventuell nicht möglichen - endgültigen Unterteilung zwischen Substantiv

102 Einzige Ausnahme bildet eine Form dieses Satzes mit der ersten Person Singular: ontemna nomaqsałam ,ich sage zu mirc. 
und Postposition wird abgesehen, stattdessen wird versucht, anhand der Realisierung des Referenzpunktes eine Kategorisierung innerhalb der Konstruktion PostP-Px vorzunehmen und diese mit strukturellen Ähnlichkeiten zur Konstruktion SuBs-Px in Relation zu setzen. Demzufolge verteilen sich die Belege der Konstruktion PозтP-Px wie folgt:

- Bei der Konstruktionsart ø PostP-Px im Ungarischen scheint es sich um die reguläre Realisierung der Konstruktion PostP-Px zu handeln. Dabei scheint ein Zusammenhang mit den affigierten Formen der Personalpronomina zu bestehen.

- Bei der Konstruktionsart PersPr PostP-Px im Ob-Ugrischen scheint es sich um die reguläre Realisierung der Konstruktion PostP-Px zu handeln, das heißt, die Konstruktion PosтP-Px wird möglicherweise im Ob-Ugrischen nur in dieser Form realisiert, das Personalpronomen ist möglicherweise obligatorisch.

- Bei der Konstruktionsart Su bs PostP-Px im Mansischen existiert möglicherweise aufgrund der Überschneidung der Wortarten ein Zusammenhang mit den Konstruktionen Subs Subs-Px (siehe Kapitel 5.2.2.2) des Mansischen. Möglicherweise ist dies auch auf das Kazym-Chantische zutreffend.

Die Hinzunahme der Textumgebung, was nun im zweiten Teil des Kapitels näher beschrieben wird, kann mitunter die hier festgestellten Tendenzen noch einmal verdeutlichen bzw. dazu beitragen, die Eigenschaften der Possessivsuffixe in den jeweiligen Konstruktionstypen genauer zu beschreiben.

\subsection{Die Konstruktionen und die Textumgebung}

Die bisherige Analyse der Vorkommen von Possessivsuffixen ist größtenteils isoliert innerhalb der jeweiligen Konstruktion und deren Bestandteile erfolgt. Hier konnten schon einige Erkenntnisse gewonnen werden, so haben sich zum Beispiel Tendenzen in der Verwendung bestimmter Realisierungen einer Konstruktion aufgezeigt (zum Beispiel bei den Postpositionen), oder bestimmte Konstruktionstypen konnten aus der weiteren Analyse ausgeschlossen werden, da das Possessivsuffix ein obligatorischer Bestandteil zu sein scheint (zum Beispiel in den Partizipialkonstruktionen mit Verben der Wahrnehmung). Insbesondere bei der Konstruktion Subs-Px jedoch wurden bestimmte Tendenzen festgestellt - so zum Beispiel im Hinblick auf die Relation Person Person, welche vom bisherigen Erklärungsansatz als Ausdruck von Possession oder Definitheit abweichen. Im folgenden Schritt soll nun die Textumgebung der Konstruktionen mit herangezogen werden, um diese Verwendungen genauer bestimmen zu können. Das bedeutet nun, abgesehen von der Tatsache, dass ein als Leerstelle oder Personalpronomen realisierter Referenzpunkt in bestimmter Hinsicht die Vorerwähnung dessen voraussetzt, dass das Vorkommen eines jeden Referenzpunktes als Parameter in die Analyse aufgenommen wird. Aber auch das Vorkommen des Targets im Text stellt einen Analyseparameter dar. Hier werden Vorerwähnung und Fortführung von Referenzpunkt und Target beider maßen berücksichtigt, aber zum Teil auch die syntaktischen und semantischen Rollen beider. Die Analyse der Textumgebung 
erfolgte dabei wie in Kapitel 4.2 beschrieben von Hand. Das bedeutet, dass sämtliche Vermerke zum Auftreten und gegebenfalls den syntaktischen und semantischen Rollen der einzelnen Referenten ohne Zuhilfenahme automatisierter Annotationsprogramme erfolgt ist. Die Analyse ist damit in ihrer Version für die vorliegende Arbeit an der Grenze des Möglichen. Dennoch ist eine gewisse Fehlerquote nicht ganz auszuschließen, welche trotz jeglicher möglicher Sorgfalt bei der Menge der zu analysierenden Belege auftreten muss. Die im Folgenden vorgestellten Ergebnisse sind als erste Kategorisierung und Übersicht gedacht, welche einen Anreiz bieten soll, die Vorkommen der Possessivsuffixe zukünftig in Richtung Informations- und Textstrukturierung gründlicher zu berücksichtigen, als dies bislang der Fall war.

Die Kategorisierung orientiert sich an der Textumgebung und ist dergestalt erfolgt, dass unter anderem die Belege in Konstruktionen mit vorerwähnten und nicht-vorerwähnten Targets unterteilt wurden. Insbesondere bei der Beschreibung der Konstruktionen mit vorerwähntem Target mussten dann weitere Kriterien zur Bestimmung einzelner Mechanismen festgelegt werden. Es wurden nicht nur die Konstruktionen der dritten Personen berücksichtigt, sondern aller Personen.

Possessivsuffixe der dritten Personen setzen das Target text-intern mit einem Referenten in Relation. Mit den Possessivsuffixen der ersten und zweiten Person hingegen wird das Target mit einem Sprechaktteilnehmer, das heißt einer text-externen Entität in Relation gesetzt, diese Unterscheidung wird zum Beispiel von Nikolaeva 2003 als »linking to a discourse-internal element" und »linking to the speech setting « bezeichnet (siehe Kapitel 2.3.4). Da es sich bei den Korpusbelegen größtenteils um Daten aus schriftlicher Erzählform handelt, entsprechen die Belege mit Possessivsuffixen der ersten und zweiten Personen hauptsächlich der text-deiktischen Verwendung. Insofern ist auch hier eine Textumgebung vorhanden (verglichen Kapitel 3.2) und solche Belege wurden daher ebenfalls strukturiert und in Mechanismen unterteilt. Besondere Berücksichtigung hierbei erfuhren die unterschiedlichen Auswirkungen je nach der Kodierung der ersten und zweiten Person. Rein deiktische Verwendungen der ersten und zweiten Personen, das heißt die Involvierung von Erzähler und Hörer, sind im Korpus der vorliegenden Arbeit nur wenige vorhanden, sie werden daher am Rande erwähnt. Eine Verknüpfung mit dem tatsächlichen Sprechaktteilnehmer, zum Beispiel dem Erzähler, kann verwendet werden, um den Hörer durch die Erzählung zu führen. Da es sich hier jedoch vorwiegend um Belege aus einem Text handelt, kann keine weitere Aussage darüber getroffen werden, ob es sich dabei um eine gängige Erzählstrategie handelt und weshalb sie zum Einsatz kommt. Es ist auch lohnend, sich mit der Textumgebung anderer Konstruktionen mit Possessivsuffixen genauer zu befassen, insbesondere bei der Konstruktion VN-Px.

Unter Mechanismus wird in der vorliegenden Arbeit die Auswirkung der Verwendung einer Konstruktion mit Possessivsuffix auf die Satz- und Textstruktur sowie auf die Sprechaktteilnehmer (das heißt deren Involvierung in die Satzaussage) verstanden. Mechanismus bedeutet nicht, dass hier eine Vereinheitlichung aller Vorkommen von Konstruktionen mit Possessivsuffixen erfolgen kann, in welcher jedem einzelnen Vorkommen ein bestimmter Mechanismus zugewiesen werden kann, welche ein Gesamtsystem mit einer in Art und Anzahl festgelegten Menge an Mechanismen ergeben. Vielmehr kann möglicherweise eine Konstruktion gleichzeitig mehrere Auswirkungen auf die Satz- und Textstruktur sowie auf die Sprechaktteilnehmer haben, 
und auch können mitunter nicht alle im Korpus auftretenden Konstruktionen berücksichtigt werden. Die im Folgenden vorgestellte Kategorisierung ist daher als Vorschlag zu verstehen, welche nicht den Anspruch erheben, als vollständig oder gebunden angesehen zu werden. Die vorliegende Arbeit unterteilt dabei zunächst drei Arten von Mechanismen - pragmatisch, syntaktisch und semantisch motivierte Mechanismen. Diese werden im Folgenden einzeln vorgestellt.

In der Kategorisierung finden neben den im Korpus angesetzten Parametern auch Beschreibungen bestimmter Verwendungen von Possessivsuffixen in der Forschung Berücksichtigung, welche anhand der eigenen Korpusdaten entweder unterstützt, wiederlegt oder re-evaluiert werden. $\mathrm{Zu}$ jedem beschriebenen Mechanismus werden die am Häufigsten in diesem Zusammenhang auftretenden und damit bei der Zuordnung auschlaggebenden Kriterien stichpunktartig zusammengefasst.

\subsubsection{Pragmatisch motivierte Mechanismen}

Den Beginn machen die Mechanismen, welche in der vorliegenden Arbeit als pragmatisch motiviert bezeichnet werden. Hierunter werden Konstruktionen zusammengefasst, deren Vorkommen nicht obligatorisch und damit von der Intention des Sprechers abhängig sind (verglichen Nikolaeva 2003: 5); das heißt, die Wahl der Possessivkonstruktion steht unter anderem mit den topikalen Rollen der in der Konstruktion in Relation gesetzten Referenten im Zusammenhang. Zunächst wird hierbei das neu erwähnte Target betrachtet, das heißt, das Target wird mit der Konstruktion im Text neu eingeführt. Durch das Vorkommen eines Targets handelt es sich hier zwangsläufig ausschließlich um das Vorkommen der Konstruktion Subs-Px, also der sogenannten Possessivkonstruktion.

\subsubsection{Anchoring}

Der erste Mechanismus, das sogenannte Anchoring, entspricht damit auch der für Possessivkonstruktionen im Allgemeinen angenommenen Grundfunktion und ist der wohl am meisten beschriebene Mechanismus von Possessivkonstruktionen in Hinsicht auf die Textstrukturierung. Beim Anchoring wird ein neuer, zuvor nicht erwähnter Referent im Textverlauf eingeführt. Um die Salienz des neuen und damit für den Hörer vermutlich unbekannten Referenten zu erhöhen, wird der Referent durch eine Verknüpfung mit einem schon etablierten und damit salienteren Referenten in einer Possessivkonstruktion verknüpft:

(59) NM_text_004_0030

\begin{tabular}{|c|c|c|c|c|c|c|c|c|c|}
\hline $\begin{array}{l}\text { sossa } \\
\text { local }\end{array}$ & $\begin{array}{l}\text { ma:xum } \\
\text { people }\end{array}$ & $\begin{array}{l}-n \\
\text {-DLAT }\end{array}$ & $\begin{array}{l}n^{j} a: l \\
\text { arrow }\end{array}$ & $\begin{array}{l}-\partial / \\
\text {-INST }\end{array}$ & $\begin{array}{l}\text { pa:xt } \\
\text { shoot }\end{array}$ & $\begin{array}{l}-u n k^{w} e \\
\text {-INF }\end{array}$ & $\begin{array}{l}\text { pat } \\
\text { begin }\end{array}$ & $\begin{array}{l}\text {-we } \\
\text {-PASS }\end{array}$ & $\begin{array}{l}-S \\
-P S T[3 S G]\end{array}$ \\
\hline
\end{tabular}

'Er kam heraus, die Hiesigen schossen mit Pfeilen auf ihn.'

(60) NM_text_004_0031

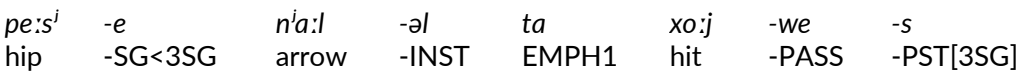

'Ein Pfeil traf seine Hüfte.' 
In der Fachliteratur ist das Anchoring unter verschiedenen Bezeichnungen bekannt: Brinker bezeichnet eine solche Textstrukturierung als »implizite Wiederaufnahme« (Brinker 2005: 36), andere Abhandlungen sprechen von der "indirekten Anapher" (»indirect anaphor « Erkü - Gundel 1987: 535) und entspricht dem Mechanismus der abgeleiteten Progression von Daneš in der Textlinguistik (verglichen Kapitel 3.2). Ein Aspekt beider Begrifflichkeiten ist meines Erachtens ausschlaggebend, diese nicht in der vorliegenden Arbeit zu verwenden: Sie erfassen die Eigenschaft nur eines der beiden beteiligten Referenten, nämlich die des Referenzpunktes. Es ist der Referenzpunkt, welcher indirekt bzw. implizit wiederaufgenommen wird bzw. auf welchen anaphorisch verwiesen wird. Somit wird das referentielle Verweismittel bezeichnet, nicht aber der Mechanismus an sich, ebenso wird keine Aussage im Hinblick auf das Target getroffen. Die Wahl des Begriffs Anchoring basiert auf der dem Mechanismus zugrunde liegenden Referenzpunkt-Konstruktion (verglichen Willemse et al. 2009: 17): Das Target wird an einem Referenzpunkt verankert, der text-strukturierende Mechanismus mittels des anaphorischen Verweismittels Possessivsuffix ist die Verankerung des Targets am Referenzpunkt. Als Anchoring sind in der vorliegenden Arbeit solche Possessivkonstruktionen klassifiziert, welche folgende Kriterien erfüllen:

- Possessivsuffix der 3. Person Singular/Dual/Plural Ein-, Dual- und Mehrbesitz

- Das Target ist neu

- Der Referenzpunkt ist vorerwähnt und wird als Leerstelle realisiert (seltener als Pronomen)

- Die Relation ist vorwiegend Ganzes Teil oder Person $\sim$ XY

Die Entscheidung, nur die dritten Personen sowie nur eine Art der Realisierung des Referenzpunktes in der Zuordnung der Konstruktionen zu dieser Funktion zu berücksichtigen, beruht auf dem Ermessen der Verfasserin, da die Fachliteratur zum hier als Anchoring bezeichneten Mechanismus darüber nicht ausreichend Auskunft gibt. Es ist anzunehmen, aufgrund der ausgeübten Funktion und den Beispielen, dass hier die dritte Person in Hinblick auf Wiederaufnahme-Mechanismen im Text eine ausschlaggebende Rolle spielt (die dritte Person Dual und Plural kann ebenfalls in der Funktion des Anchoring angenommen werden, in diesem Fall dient eine Gruppe von salienten Referenten als Anker). Die Formen des Dual- und Mehrbesitz werden ebenfalls als möglich beim Anchoring angesehen, wenn es sich um eine Gruppe von neuen Targets handelt. Die vorausgesetzte Vorerwähntheit des Referenzpunktes entspricht in den ugrischen Sprachen meist einer Realisierung als Leerstelle in der Konstruktion, sodass diese zwei Voraussetzungen für den Mechanismus des Anchoring meines Erachtens schlüssig sind. Anhand dieser Kriterien sind 513 Token im Korpus der Funktion Anchoring zuzuordnen.

Damit repräsentiert die Funktion einen viel geringeren Anteil der Verwendungen, als vielleicht aufgrund der Beschreibungen zu erwarten war (siehe Zitat Taylor 1996). Das Anchoring ist demnach nicht die primäre Funktion der Possessivkonstruktionen in den ugrischen Sprachen.

Das dem Anchoring zugrunde liegende Prinzip ist auch in grundlegenden Abhandlungen zur Possession im Rahmen der kognitiven Linguistik zu finden, verglichen Taylor 1996 (siehe Kapitel 3.1). Des Weiteren wird beschrieben, dass das Anchoring auf 
semantischer Kontiguität beruht, wie es zum Beispiel bei Ganze S TEIL Relationen der Fall ist. Diese machen jedoch nur gut die Hälfte der Anchoring-Belege im Korpus aus. Erkü/Gundel 1987 beschreiben außerdem die »created indirect anaphora «, eine Art des Anchoring, welche nicht durch inhärente semantische Eigenschaften ausgelöst ist, sondern aufgrund des Handlungsverlaufs erfolgt (Erkü/Gundel 1987: 539). Das Anchoring an einen in den Handlungsverlauf involvierten Referenten könnte mit der Relation PERson im Zusammenhang stehen, welche in etwa die zweite Hälfte aller Belege ausmacht.

Bedeutend weniger Belege weisen, entsprechend der Verteilung im Allgemeinen, einen pronominalen Referenzpunkt auf. Von den 25 Belegen entfallen jedoch lediglich sechs auf die ob-ugrischen Sprachen und beim Großteil der ungarischen Vorkommen handelt es sich um Demonstrativ- und Relativpronomina im Dativ, sodass die Realisierung eher einer konstruktionsbedingten Platzhalterfunktion entspricht; das heißt, der Referenzpunkt wird deshalb nicht als Leerstelle realisiert, da die Relativsatzkonstruktion ein Pronomen anstatt einer Leerstelle verlangt. Diese Belege könnten somit ebenfalls im weitesten Sinne zum Anchoring gezählt werden.

Ähnlich wie beim text-internen Anchoring erhöht die Verankerung an einem der Sprechaktteilnehmer die Salienz eines neuen Targets. Die Sprechakteilnehmer sind deswegen als Referenzpunkte geeignet, da sie inhärent aktiviert sind (verglichen Kapitel 3.2). Dabei muss jedoch unterschieden werden, mit welchem der beiden Sprechaktteilnehmer das Target verankert wird. Wählt der Sprecher sich selbst als Referenzpunkt, das heißt, werden die Possessivsuffixe der ersten Personen verwendet, so spricht man unter anderem von der hörer-indefiniten Referenz (Kolde 1989: 84f.), was bedeutet, dass die Origo des Sprechers herangezogen wird, um darüber die Salienz eines Targets für den Hörer zu erhöhen.

(61) HU_text_008_0081

\begin{tabular}{|c|c|c|c|c|c|}
\hline $\begin{array}{l}\text { bátyá } \\
\text { elder brother }\end{array}$ & $\begin{array}{l}-m \\
-S G<1 S G\end{array}$ & $\begin{array}{l}\text { olyan } \\
\text { such }\end{array}$ & $\begin{array}{l}\text { okos } \\
\text { smart }\end{array}$ & $\begin{array}{l}\text { vol } \\
\text { to be }\end{array}$ & $\begin{array}{l}-t \\
-\mathrm{PST}\end{array}$ \\
\hline
\end{tabular}

'Mein Bruder war so klug.'

Wählt der Sprecher hingegen sein Gegenüber, also den Hörer, als Referenzpunkt, so ist anzunehmen, dass für ihn selbst das Target weniger salient ist als für den Hörer. Über die Verankerung mit diesem kann der Sprecher das Target für sich aktivieren.

(62) HU_text_009_0018

ha csak az a baj -od

if only that the problem $-S G<2 S G$

'Wenn nur das dein Kummer ist.'

Analog wird dieses Verfahren als sprecher-indefinite Referenz bezeichnet (Kolde 1989: 84f.). Da beide Termini mit dem Begriff Definitheit operieren, wird von einer Übernahme in der vorliegenden Arbeit abgesehen, stattdessen wird in beiden Fällen vom text-externen Anchoring gesprochen. Dieses hat folgende Merkmale:

- Possessivsuffix der 1./2. Person Singular/Dual/Plural Ein-, Dual- und Mehrbesitz

- Das Target ist neu

- Der Referenzpunkt ist vorerwähnt und wird als Leerstelle realisiert (seltener als Pronomen)

- Die Relation ist vorwiegend Person $\sim$ XY oder GANZES TeIL 
Diese machen insgesamt 170 Vorkommen im Korpus aus, wobei die Belege mit Possessivsuffixen der ersten Personen die der zweiten Personen um ein vielfaches übersteigen (125 gegenüber 45 Belegen). Es ist außerdem darauf hinzuweisen, dass es sich nur bei zehn Vorkommen um eine tatsächliche Verknüpfung mit den Sprechaktteilnehmern, das heißt Erzähler und Zuhörer, handelt. Beim Großteil handelt es sich um text-deiktische Verwendung, das heißt, der Mechanismus wird innerhalb des Textes im Dialog oder Ähnlichem eingesetzt. Ähnlich wie beim text-internen Anchoring machen die Relationen Ganzes Teil und mit Person den größten Anteil an Relationen aus, das Verhältnis beider ist jedoch umgekehrt zueinander. Der Referenzpunkt wird vorwiegend als Leerstelle realisiert, den Rest machen pronominale Erwähnungen (inklusive emphatischer Personalpronomina) aus, lexikalische Erwähnungen schließen sich ohnehin aus.

\subsubsection{Re-Aktivierung}

Der Großteil der Possessivkonstruktionen, rund zwei Drittel aller Vorkommen, weist ein bereits vorerwähntes Target auf. So ist zum Beispiel in der in Kapitel 5.1.1.4 beschriebenen PERson Person Relationen ebenfalls lediglich in 76 der 950 Belegen das Target nicht vorerwähnt - ein Aspekt, der ebenfalls dafür spricht, dass es weniger um den Ausdruck bestimmter Verwandtschaftsverhältnisse geht, sondern um die Strukturierung der Handlung zweier Partizipanten im Textverlauf (verglichen auch hier »created indirect anaphora « Erkü/Gundel 1987: 539). Wenn nun nicht die Einführung neuer Referenten das Hauptkriterium für das Vorkommen einer Possessivkonstruktion ist, weshalb werden Referenten dann als Targets in einer Possessivkonstruktion realisiert? Bei den vorerwähnten Targets kann es sich beispielsweise um eine Art der Re-Aktivierung handeln.

Zieht man für eine solche Re-Aktivierung diejenigen Targets in Betracht, zwischen deren Wieder-Einführung mit der Possessivkonstruktion und der letzten Vorerwähnung mehr als ein Satz liegt, entsprechen allein in den dritten Personen mehr als 800 Belege der Re-Aktivierung. Sie bilden damit die größte Gruppe der Possessivkonstruktionen. Je kürzer dabei die Vorerwähnung des Referenzpunktes zurückliegt und gleichzeitig je länger die Vorerwähnung des Targets zurückliegt, desto mehr ähnelt die Funktion der Re-Aktivierung dem Anchoring: ein salienter, da in der momentanen Handlung involvierter Referenzpunkt dient als Anker, um einen weniger salienten, da länger nicht in der Handlung involviertes Target wieder zu aktivieren. Dieser Re-Aktivierung entsprechen rund 500 Belege im Korpus, die längste Vorerwähnung eines Targets entspricht dabei 222 Sätzen, was einer Neuerwähnung beinahe gleichkommt. Des Weiteren sind Belege im Korpus enthalten, in welchen die Vorerwähnung sowohl vom Referenzpunkt als auch vom Target länger als zwei Sätze zurückliegen (»look-back« = »im Text«). Diese machen einen weitaus geringeren Anteil aus (rund 100 Belege), bestätigen aber auch, dass die Salienz des Referenzpunktes so etabliert sein muss, dass sie selbst dann noch ausreicht, um ein Target daran zu verankern, wenn der Referenzpunkt selbst länger inaktiv war. Dies spricht ebenfalls für die hohe referentielle Verweiskraft des Possessivsuffixes, zumal der Referenzpunkt in drei Viertel der Vorkommen als Leerstelle realisiert ist. 
Die Gruppe von Belegen, in welchen das Target unmittelbar aus dem vorangehenden Satz re-aktiviert wird, ist um einiges geringer (etwa 180 Belege). Somit liefert die Unterteilung der Vorerwähnung des Targets in Vorerwähnungen im Satz und Vorerwähnungen im Text allein keine besonders aussagekräftigen Anhaltspunkte - die große Gruppe der Vorerwähnungen im Text könnte mit zum Anchoring gerechnet werden, während für die kleinere Gruppe der Vorerwähnungen im Satz keine weitere Aussage getroffen werden kann, außer dass diese nicht zum Anchoring gezählt werden kann. Somit reicht es auch nicht aus, lediglich zwischen neuen und vorerwähnten Targets zu trennen:

We will propose that [...] the question of the discourse status of PM referents of possessive NPs cannot be reduced to a binary distinction between new or given in the discourse. (Willemse et al. 2009: 13)

Neben dem Ort ist damit die Art der Vorerwähnung ein ausschlaggebendes Kriterium: Wird das Target im Text oder Satz zuvor erwähnt, kann dies alleine oder gemeinsam in einer syntaktischen Rolle mit dem Referenzpunkt erfolgen. Diese Gruppe wird im Folgenden von den anderen Vorerwähnungen gesondert analysiert. Zuvor soll noch versucht werden, die übrigen vorerwähnten Targets mithilfe weiterer Analyseparameter genauer zu strukturieren. Wichtige Anhaltspunkte sind hierbei die Fortführung des Targets sowie die syntaktischen und semantischen Rollen von Referenzpunkt und Target. Dabei wird von der in Kapitel 3.2 erwähnten Topik-Subjekt-Persistenz ausgegangen, das heißt, dass die höchste topikale Rolle gleichzeitig die höchste syntaktische Rolle im Satz ausübt.

\section{Topic Chain}

Wird das Target nur für den aktuellen Satz re-aktiviert und ist jedoch im Folgesatz nicht weiter in die Handlung involviert, so ist eher davon auszugehen, dass in diesen Fällen die Kontinuität eines bestimmten Topiks (des Diskurs-Topiks) im Text verwendet wird, um ein Target, welches kurzzeitig wieder in der Handlung mit aufgegriffen wird, daran zu verankern. Der kodierte Referenzpunkt ist somit Teil einer topikalen Kette. Dafür spricht auch, dass der Referenzpunkt zum einen in den meisten Fällen im unmittelbar vorangehenden Satz erwähnt wird, nur selten liegt die Vorerwähnung länger zurück. Zieht man die syntaktischen und semantischen Rollen hinzu, in welchen Referenzpunkt und Target realisiert werden, so lassen sich drei Gruppen bilden:

(a) Das Target wird als adverbiale Ergänzung (vorwiegend als Goal/Adressee) realisiert (203 Belege), der Referenzpunkt ist meist gleichzeitig Subjekt und Agens.

(63) HU_text_001_0002

\begin{tabular}{|c|c|c|c|c|c|c|c|}
\hline $\begin{array}{l}\text { egy } \\
\text { one }\end{array}$ & $\begin{array}{l}\text {-szer } \\
\text {-ITER }\end{array}$ & $\begin{array}{l}a \\
\text { the }\end{array}$ & $\begin{array}{l}\text { szegény } \\
\text { poor }\end{array}$ & & $\begin{array}{l}\text { ember } \\
\text { man }\end{array}$ & $\begin{array}{l}a z \\
\text { that }\end{array}$ & $\begin{array}{l}-t \\
-A C C\end{array}$ \\
\hline mond & $\begin{array}{l}-0 \\
-P R S\end{array}$ & $\begin{array}{l}-j a \\
-s\end{array}$ & & the & fi & $\begin{array}{l}-a ́ \\
-S G<3 S G\end{array}$ & -nak \\
\hline
\end{tabular}

'Einmal sagte der arme Mann zu seinem Sohne.'

(b) Das Target wird als Subjekt und Agens (seltener Patiens) realisiert (177 Belege). 
(64) KK_text_010_0004

$\begin{array}{lllll}\text { ike } & -4 & \text { sorma pit } & - \text {-əm } & \text { jupijn } \\ \text { husband } & - \text { SG }<3 \text { SG } & \text { die } & \text {-PTCP.PST } & \text { after }\end{array}$

'Nachdem der Mann gestorben war.'

(c) Das Target wird als direktes Objekt und Patiens realisiert (139 Belege), der Referenzpunkt ist in den meisten Fällen gleichzeitig Subjekt des Satzes.

(65) NM_text_007_0022:1

\begin{tabular}{|c|c|c|c|c|c|}
\hline $\begin{array}{l}\text { it'i } \\
\text { at night }\end{array}$ & $\begin{array}{l}\text { ul'a } \\
\text { fire }\end{array}$ & $\begin{array}{l}-t e \\
-S G<3 S G\end{array}$ & $\begin{array}{l}\text { nox- } \\
\text { up }\end{array}$ & $\begin{array}{l}\text { palt } \\
\text { start a fire }\end{array}$ & $\begin{array}{l}\text {-unk } \\
\text {-INF }\end{array}$ \\
\hline
\end{tabular}

'In der Nacht stand er auf, um sein Feuer anzuheizen.'

Gruppe (a) und (c) weisen damit eine niedrigere Position für das Target in der Hierarchie nach Givón auf (verglichen Tabelle 12) und stehen außerdem in Relation mit dem Referenzpunkt, was ebenfalls für eine Fortführung der topikalen Kette spricht. Da überdies in circa drei Viertel aller Belege mit dem Target in einer Subjektposition der Referenzpunkt unmittelbar im Satz zuvor oder im Folgesatz wieder die Rolle des Subjekts übernimmt und die Re-Aktivierung des Targets somit ebenfalls nur kurzzeitig erfolgt, werden die Belege der Gruppe (b) ebenfalls als Beibehaltung einer topikalen Kette interpretiert. Eine andere Wertung mit weiteren Analysemethoden kann dennoch nicht ausgeschlossen werden.

Wird das Target im Folgesatz hingegen fortgeführt, so lassen sich diese Vorkommen wiederum in zwei Gruppen teilen:

(a) Der Referenzpunkt wird fortgeführt, die Topik-Subjekt Persistenz bleibt dabei gleich; das heißt, es handelt sich fast ausschließlich um Belege, in welchen der Referenzpunkt in einer syntaktischen Kernrolle und das Target in einer dazu in Relation stehenden syntaktischen Kernrolle oder adverbialen Ergänzung steht.

(b) Die Topik-Subjekt-Persistenz ändert sich zugunsten des Targets.

Somit ist bei Gruppe (a) ebenfalls eher von der Beibehaltung einer topikalen Kette auszugehen. Diese Vorkommen werden daher gemeinsam als ein Mechanismus unter der Bezeichnung Topic Chain geführt. Die Benennung fokussiert die Auswirkung auf den Referenzpunkt, da eine Benennung ausgehend von den Auswirkungen auf das Target (Re-Aktivierung) nicht die Unterschiede zwischen kurzzeitiger Erwähnung und Fortführung beinhaltet. Der Mechanismus Topic Chain weist folgende Charakteristiken auf:

- Possessivsuffix 3. Person Singular/Dual/Plural, Ein, Dual- und Mehrbesitz

- Das Target ist vorerwähnt

- Der Referenzpunkt ist vorwiegend als Leerstelle realisiert

- Die Hierarchie zwischen Target und Referenzpunkt entspricht im vorausgehenden sowie im Folgesatz der bisherigen Topik-Subjekt-Persistenz

- Die Relation entspricht häufig PERson PERSON

Nur wenige Belege, welche zum Topic Chain gezählt werden können, weisen eine Realisierung des Referenzpunktes in der Konstruktion als Substantiv oder Pronomen auf. Wie zuvor beschrieben reicht die Leerstelle zusammen mit dem Possessivsuffix durch- 
aus aus, um das Target zu verankern. Zudem ist die Realisierung des Referenzpunktes in den ob-ugrischen Sprachen in der Possessivkonstruktion mit Possessivsuffix eher untypisch. Tatsächlich finden sich in den ob-ugrischen Belegen wieder emphatische Pronomina und im Ungarischen die Relativpronomina, welchen zuvor eine Platzhalterfunktion attestiert wurde.

\section{Topic Shift}

Die Vorkommen, welche übrig bleiben, wenn sowohl nicht fortgeführte Targets als auch die Fortführung von Targets unter Beibehaltung der Topik-Subjekt-Persistenz zum Topic Chain gezählt werden, machen dementsprechend ausschließlich Belege aus, in welchen das Target die Subjektrolle übernimmt. Wird der Referenzpunkt weitergeführt, dann in einer veränderten Topik-Subjekt-Persistenz (zum Beispiel als direktes Objekt) oder gemeinsam mit dem Target in der Subjektposition, und auch dann erfolgt eine Promotion des Targets. Damit ist eine weitere Annahme zu den Possessivkonstruktionen im Allgemeinen, dass Targets keine Topikalität aufweisen können, nicht für die ugrischen Sprachen und insbesondere für die ob-ugrischen zu belegen:

We see that possessees overwhelmingly introduce new, previously unnamed entities into the discourse. [...] Furthermore, possessee entities are nearly always immediately dropped from the discourse. In other words, possessee entities are not only non-topical at the time of their mention, they also fail to achieve topicality in following discourse. (Taylor 1996: 217)

Gleichzeitig mit der Fortführung des Targets erfolgt ein Wechsel der Referenten im weiteren Handlungsverlauf, die Textstrukturierung ähnelt dabei einem Switch-ReferenceMechanismus. ${ }^{103}$ Dieser Mechanismus wird daher analog zur Topic Chain als Topik Shift bezeichnet.

(66) NM_text_007_0030:2

$\begin{array}{llllll}\text { s'owal } & \text { sunt } & - \text { nal } & \text { jolal }^{i} & \text { xuntl } & -i \\ \text { hearth } & \text { opening } & - \text { ABL } & \text { downwards } & \text { look } & \text {-PRS[3SG] }\end{array}$

'Er kletterte auf das Dach des Hauses und sah durch den Schornstein hinunter.'

(67) NM_text_007_0031:1

\begin{tabular}{|c|c|c|c|c|c|c|c|}
\hline \multicolumn{2}{|c|}{$\begin{array}{l}\text { sujpil } \\
\text { lingonberry }\end{array}$} & $\begin{array}{l}\text { lupta } \\
\text { leaf }\end{array}$ & $\begin{array}{l}\text { panxwit } \\
\text { in width of }\end{array}$ & $\begin{array}{l}\text { joso } \\
\text { ski }\end{array}$ & $\begin{array}{l}-\eta \\
-A D J Z R\end{array}$ & $\begin{array}{l}\text { xumi } \\
\text { man }\end{array}$ & $\begin{array}{l}-t e \\
-S G<3 S G\end{array}$ \\
\hline $\begin{array}{l}s^{j} a n \\
\text { mother }\end{array}$ & $\begin{array}{l}-e \\
-S\end{array}$ & $3 S G$ & $\begin{array}{l}\text { nupəl } \\
\text { towards }\end{array}$ & $\begin{array}{l}\text { potart } \\
\text { tell }\end{array}$ & $\begin{array}{l}-i: \\
-P R S[3 S G]\end{array}$ & & \\
\hline
\end{tabular}

'Der Mann mit Skiern klein wie Preiselbeerblätter erzählte seiner Mutter:'

(68) NM_text_007_0031:2

\begin{tabular}{|c|c|c|c|c|c|c|c|}
\hline $\begin{array}{l}\text { akw } \\
\text { one }\end{array}$ & $\begin{array}{l}\text { ojka } \\
\text { man }\end{array}$ & $\begin{array}{l}\text { jot } \\
\text { with }\end{array}$ & $\begin{array}{l}\text { wor } \\
\text { forest }\end{array}$ & $\begin{array}{l}-t \\
-\mathrm{LOC}\end{array}$ & $\begin{array}{l}\text { xul } \\
\text { stay }\end{array}$ & $\begin{array}{l}-s \\
-P S T\end{array}$ & $\begin{array}{l}-u m \\
-1 S G\end{array}$ \\
\hline
\end{tabular}

'Ich habe mit einem Mann im Wald übernachtet.'

103 Switch-Reference bezeichnet eine obligatorische Markierung bei Topik-Wechseln in manchen Sprachen. Die ugrischen Sprachen sind demnach keine Switch-Reference-Sprachen, scheinen jedoch anaphorische Verweismittel im reference-tracking dergestalt einsetzen zu können, dass neben der Fortführung auch der Wechsel von topikalen Referenten angezeigt werden kann. 
Wie in allen anderen Konstruktionen mit Possessivsuffixen wird auch beim Topic Shift von der Verankerung des Targets an einem topikalen Referenzpunkt ausgegangen, welcher im Possessivsuffix kodiert wird. Folglich wird als Referenzpunkt der in den vorangehenden Sätzen die Subjektrolle ausübende Referent angenommen. Dennoch kann eine gleichzeitige gewisse hinweisende Funktion des Possessivsuffixes der dritten Person Singular auf den neuen Referenten in Subjektposition nicht von der Hand gewiesen werden. Diese ähnelt beispielsweise einer Art Hervorhebung (»emphasis«) oder Kontrastierung, welche beispielsweise Nikolaeva 2003 für das Possessivsuffix der dritten Person Singular der uralischen Sprachen im Wolga-Kama-Areal beschreibt (Nikolaeva 2003: 11f.). Möglicherweise findet sich dieser Mechanismus daher an der Grenze zum Wandel des rückverweisenden Personalmarkers zu einer hinweisenden Fokussierungs-Partikel. Auch dieser Kontrast zwischen der Beibehaltung des Referenzpunktes als Topik und dem Hinweis auf den Wechsel zum Target als neuem Topik ist durch die Bezeichnungen Topic Chain und Topic Shift hervorgehoben. Kriterien für den Topic Shift sind die Folgenden:

- Possessivsuffix der 3. Person Singular/Dual/Plural Ein-, Dual- und Mehrbesitz

- Das Target ist vorerwähnt

- Der Referenzpunkt ist vorerwähnt

- Der Referenzpunkt wird als Leerstelle in der Konstruktion realisiert

- Der Referenzpunkt wird nicht fortgeführt

- Das Target wird als Subjekt fortgeführt

- Die Relation ist fast ausschließlich Person Person

\section{Re-Aktivierung in den ersten und zweiten Personen}

Auch die Sprechaktteilnehmer werden mitunter herangezogen, um bereits vorerwähnte Targets zu re-aktivieren. Möglicherweise wird der Referenzpunkt - erste oder zweite Person - hier ebenfalls dementsprechend eingesetzt, welcher der Sprechaktteilnehmer zuvor mit dem Target durch die Handlung in Relation gebracht wurde. Beispiel (69) verknüpft einen Ort mit dem Hörer, ein Relativsatz formuliert dabei die Relation aus. Der Sprecher re-aktiviert den Ort, indem er aufgrund der Handlung des Hörers dort mit diesem verknüpft.

(69) KK_text_009_0054

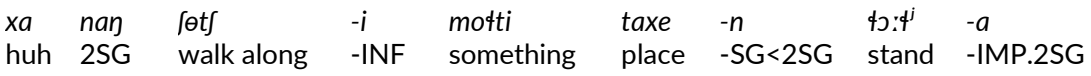

'Ha, wenn das dein Wanderweg ist, dann stehe hier eben.' (wörtl. 'Dein entlanggehender Ort.')

Eine genauere Bestimmung ist jedoch im Rahmen der vorliegenden Arbeit kaum möglich, da hierfür zusätzlich zur Vorerwähnung und Fortführung sämtliche Referenten, welche mit dem Target zuvor durch den Handlungsverlauf in Relation gestanden haben, zu bestimmen. Stattdessen können eventuell die Vorkommen der zweiten Personen in den Sätzen aus der Feldforschung herangezogen werden, um diese Vermutung zu erhärten: Dort wird auf Referenten aus dem Stimulus vorwiegend mit den Possessivsuffixen der zweiten Personen verwiesen, da sie für den Sprecher nicht salient sind, für den Hörer hingegen insofern, als es sich um dessen Stimulus handelt. 
(70) KK_satz_0026 $6^{104}$

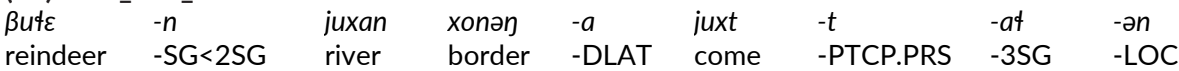

'Als das Rentier ans Flussufer kommt.'

Die Verknüpfung mithilfe der ersten Personen könnte im Gegenzug eine Strategie sein, die an sich unbekannten Referenten mit Zuhilfenahme vergleichbarer Entitäten, zu welchen der Sprecher einen Bezug hat, salienter zu machen.

(71) KK_satz_0050

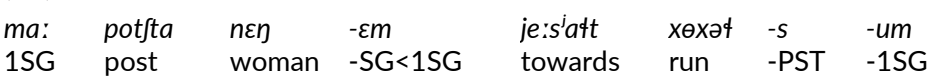

'Ich lief der Postbotin entgegen.'

Für weitere Beschreibungen sind jedoch die Daten nicht ausreichend, weswegen darauf nur am Rande verwiesen wird.

Die 195 Belege des Topic Chain in den ersten und zweiten Personen setzen sich zusammen aus (a) Targets, welche im Folgesatz nicht fortgeführt werden, und (b) fortgeführten Targets ohne Auswirkung auf die Topik-Subjekt-Persistenz. Die Targets, welche nicht fortgeführt werden (a), lassen sich wie die Targets, welche in Possessivkonstruktionen mit dritten Personen vorkommen, in drei Gruppen unterteilen:

(a) Das Target wird als adverbiale Ergänzung (vorwiegend als Goal/Adressee) realisiert (61 Belege), der Referenzpunkt ist meist gleichzeitig Subjekt und Agens.

(72) KK_text_005_0017

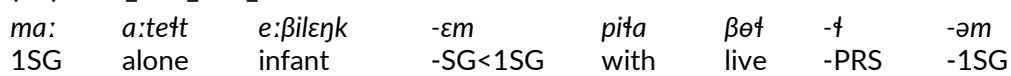

'Ich lebe alleine mit meinem Töchterchen.'

(b) Das Target wird als Subjekt und Agens (seltener Patiens) realisiert (72 Belege).

(73) KK_text_006_0075

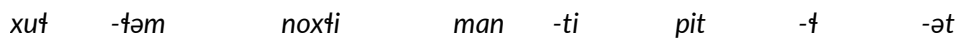

fish $\quad-P L<1 S G$ upwards go - INF start $\quad$-PRS $-3 P L$

'Meine Fische fangen schon an, aus dem Wasser zu springen.'

(c) Das Target wird als direktes Objekt und Patiens realisiert (59 Belege), der Referenzpunkt ist in den meisten Fällen gleichzeitig Subjekt des Satzes.

(74) NM_text_010_0074:3

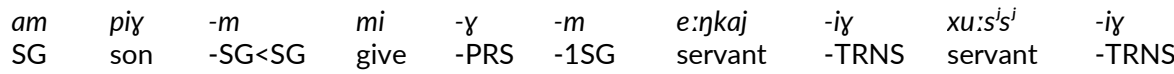

'Ich gebe inm meinen Sohn als Arbeiter, als Diener.'

Die Verteilung unterscheidet sich jedoch, nicht die adverbialen Ergänzungen sind die häufigste Funktion der Targets, sondern die Subjektfunktion. Auch die Relation der Targets, welche als direktes Objekt realisiert werden, erhöht sich im Vergleich zur adverbialen Ergänzung. Beides entspricht der generell erkennbaren Tendenz der ugrischen Sprachen, belebte Referenten (das heißt Sprechaktteilnehmer) eher in syntaktischen

104 Die Übersetzungen von Beispielen 70 und 71 entsprechen in etwa dem Stimulus, das heißt, die Possessivsuffigierung wurde vom Informant hinzugesetzt und war nicht durch die Elizitierung beabsichtigt. 
Kernrollen zu realisieren als in adverbialen Ergänzungen (die Adressierungs-Funktion ausgenommen).

Wird das Target im Folgesatz hingegen fortgeführt, so lassen sich auch die Vorkommen der Possessivsuffixe der ersten und zweiten Personen wiederum in zwei Gruppen teilen:

(a) Der Referenzpunkt wird fortgeführt, die Topik-Subjekt-Persistenz bleibt dabei gleich; das heißt, es handelt sich fast ausschließlich um Belege, in welchen der Referenzpunkt in einer syntaktischen Kernrolle, das Target in einer dazu in Relation stehenden syntaktischen Kernrolle oder adverbialen Ergänzung steht.

(b) Die Topik-Subjekt-Persistenz ändert sich zugunsten des Targets.

In allen drei Personen zusammengerechnet können dem Topic Chain 738 Belege zugeordnet werden. Das Mansische weist die meisten Belege des Topic Chain auf (303 Belege), gefolgt vom Ungarischen (197 Belege). Die chantischen Dialekte machen zusammen 238 Belege aus, wobei lediglich 96 auf das Surgut-Chantische entfallen. In Relation zu den Vorkommen von Possessivsuffixen insgesamt entspricht dies im Surgut-Chantischen jedoch fast einem Drittel der Belege, im Ungarischen nur einem Fünftel.

Dem Topic Shift können 224 Belege insgesamt zugeordnet werden. Lediglich $31 \mathrm{Be}-$ lege entfallen davon auf das Ungarische. Fast die Hälfte der Vorkommen entfallen auf das Mansische (107 Belege), 56 auf das Kazym-Chantische und knapp halb so viele auf das Surgut-Chantische (30 Belege). Die Verwendung der Possessivsuffixe als Marker eines Topic Shifts scheint daher eher ein Mechanismus der ob-ugrischen Sprachen zu sein.

\section{Anrede}

Abschließend soll noch erwähnt werden, dass die Possessivsuffixe der ersten Personen vielfach zur Anrede eingesetzt werden, ebenfalls in einer text-deiktischen Funktion. Das Target, das Gegenüber des Sprechers, muss dabei im Text vorerwähnt sein und wird direkt angesprochen, das Prädikat ist demnach häufig in der zweiten Person, mitunter im Imperativ.

(75) NM_text_012_0097

\begin{tabular}{|c|c|c|c|c|c|c|}
\hline $\begin{array}{l}S \mathrm{SG} \\
\mathrm{s}\end{array}$ & $\begin{array}{l}\text { o:jka } \\
\text { old man }\end{array}$ & $\begin{array}{l}\text { xunt } \\
\text { backpack }\end{array}$ & $\begin{array}{l}\text {-ən } \\
\text {-DLAT }\end{array}$ & $\begin{array}{l}\text { nonx- } \\
\text { up }\end{array}$ & $\begin{array}{l}\text { talt } \\
\text { put on a sledge }\end{array}$ & \\
\hline
\end{tabular}

'My uncle, put your backpack up on the sledge.'

Zum Vergleich, bei den neu erwähnten Targets, welche mit den Possessivsuffixen der ersten und zweiten Personen versehen sind, findet sich kein einziger Beleg, in welchem das Prädikat nicht in der dritten Person steht. Hier wird also über einen Referenten gesprochen, welcher selbst daher in der dritten Person ist und mit einem Sprechaktteilnehmer verankert ist.

Der Anrede entsprechen 118 Belege, die Kriterien sind:

- Possessivsuffix der 1. Person Singular/Dual/Plural Ein-, Dual- und Mehrbesitz

- Das Target ist vorerwähnt

- Der Referenzpunkt ist vorerwähnt

- Der Referenzpunkt wird vorwiegend als Leerstelle in der Konstruktion realisiert 
- Das Target fungiert als adverbiale Ergänzung (Adressee), das Prädikat steht häufig im Imperativ

- Die Relation ist fast ausschließlich PERSON PERson

Bei der Anrede wird das Target nicht nur mit dem Sprecher verknüpft, es ist auch in die Handlung involviert. Dieser Mechanismus wird unter anderem auch von Nikolaeva 2003 beschrieben (verglichen Nikolaeva 2003: 6). Auch Schlachter 1960 erwähnt mit dem »Vokativ« einen ähnlichen Mechanismus im Komi-Syrjänischen (Schlachter 1960: 23). Dieser Mechanismus unterscheidet die erste Person noch einmal von der Verwendung der Sprechaktteilnehmer als Referenzpunkt insgesamt.

\subsubsection{Gemeinsame Vorerwähnung von Referenzpunkt und Target}

Eine besondere Gruppe unter den Belegen mit vorerwähntem Target machen solche aus, in welchen Referenzpunkt und Target gemeinsam vorerwähnt wurden, entweder indem sie zusammen im vorangehenden Satz in der Subjektposition standen oder indem Referenzpunkt und Target ihre Rollen im Vergleich zum Folgesatz gewechselt haben.

\section{Turn-Taking}

Wird die Rolle gewechselt, wird dabei auf einen Referenten mithilfe einer Possessivkonstruktion in einem Satz referiert, dieser Referent wird nun im Folgesatz zum Referenzpunkt einer weiteren Possessivkonstruktion, in welcher auf den Referenten als Target Bezug genommen wird, der zuvor der Referenzpunkt war.

(76) KK_text_008_0023

\begin{tabular}{|c|c|c|c|c|c|c|}
\hline ikile & -4 & if- & of & -əS & imile & -4 \\
\hline little man & $-S G<3 S G$ & down & lie & -PST[3SG] & mistress & $-S G<3 S G$ \\
\hline tot & - tał & pasan & -a & faßi & & -adte \\
\hline & $-P L<3 S G$ & table & -DLAT & arrange & -PST & $-\mathrm{PL}<3 S \mathrm{SG}$ \\
\hline
\end{tabular}

'Der Mann legte sich nieder, die Frau ordnete die Speisen auf dem Tisch.'

Beide Referenten wechseln sich somit im Handlungsverlauf ab, der Wechsel wird mithilfe von Possessivkonstruktionen strukturiert.

Damit ist die semantische Relation zwangsläufig wieder eine PERson Person Relation, welche durch den gemeinsamen Handlungsverlauf (entweder durch Interaktion oder einen Dialog) entsteht und weniger die verwandtschaftliche Relation fokussiert (beide Referenten können jedoch gleichzeitig in einer solchen Relation zueinander stehen). Vielmehr kann dem Possessivsuffix wiederum eine hinweisende Funktion ähnlich der beim Topic Shift attestiert werden. Bis auf wenige Ausnahmen ist es das Possessivsuffix der dritten Person Singular Einbesitz. Das Auffinden solcher Strukturen im Textverlauf gestaltet sich oft schwierig, da nicht nur nach einer Abfolge von Possessivkonstruktionen mit einem entsprechenden Wechsel von Target und Referenzpunkt gesucht werden muss, sondern diese auch mitunter von den Dialogen, welche sie einleiten, unterbrochen wird. Eine weitere Frage erhebt sich, ob lediglich direkt aufeinanderfolgende Wechsel diesem Mechanismus zugeschrieben werden sollen oder auch länger zurückliegende Wechsel und wenn ja, unter welchen Kriterien. Die Hauptkriterien sind die Folgenden: 
- vorwiegend Possessivsuffix der 3. Person Singular Einbesitz

- Das Target und der Referenzpunkt werden gemeinsam vorerwähnt

- Das Target entspricht bei der Vorerwähnung dem Referenzpunkt

- Der Referenzpunkt entspricht bei der Vorerwähnung dem Target

- Der Referenzpunkt wird als Leerstelle in der Konstruktion realisiert

- Die Relation ist Person Person

So können 28 Belege zu dieser Gruppe gezählt werden. Nimmt man jedoch diejenigen Belege des Mechanismus der Anrede hinzu, welche eine ähnliche Abwechslung zwischen Adressierendem und Adressaten aufweisen, so verdoppelt sich die Anzahl. Erwähnenswert hierbei ist auch die Distribution zwischen den beiden Sprachzweigen: Während das Surgut-Chantische kaum einen Beleg des Turn-Taking aufweist, entfallen nahezu alle Belege des Turn-Taking in der dritten Person auf das Mansische und Kazym-Chantische. Das Ungarische hingegen weist fast keinen Beleg in der dritten Person auf, macht jedoch den Großteil der Belege mit erster Person aus. Möglicherweise ließen sich somit zweierlei Verwendungen eines ähnlichen Mechanismus für das Ungarische einerseits und die ob-ugrischen Sprachen (evtl. ohne das Surgut-Chantische) festhalten. Leider ist die Datenmenge hierzu jedoch zu gering.

\section{Known Group}

Das erste Vorkommen, die gemeinsame Erwähnung von Referenzpunkt und Target in Subjektposition im vorangehenden Satz, wird in der vorliegenden Arbeit angelehnt an Simonenko 2014 als »Known Group« bezeichnet (Simonenko 2014: 4). Dieser Mechanismus wird auch von Nikolaeva 2003 als Unterart der bei ihr als »Identifiability based on anaphora « bezeichneten Funktion der Possessivsuffixe beschrieben (Nikolaeva 2003: 5). Grundlegend für diesen Mechanismus ist zunächst das Aufgreifen eines Referenten im Folgesatz mit einer Possessivkonstruktion.

(77) NM_text_008_0001

$\begin{array}{llll}\text { kit } & \text { jo:rn } & \text { o:l } & - \text { er } \\ \text { two } & \text { Samoyede } & \text { live } & \text {-PRS[3DU] }\end{array}$

'There are two Samoyedes.'

(78) NM_text_008_0002

$\begin{array}{lllll}\text { janiy } & \text { xumi } & - \text { te } & \text { ne: } & -\eta \\ \text { elder } & \text { man } & \text {-SG<3SG } & \text { woman } & - \text { ADJZR }\end{array}$

'The older man is married.'

(79) NM_text_008_0003

$m a: n^{j}$ xumi -te ne: -ta:l

young man $-S G<3 S G$ woman $-A B E$

'Der jüngere Mann ist unverheiratet.'

Da die Verwendung der Possessivkonstruktion jedoch nicht obligatorisch zu sein scheint, geht Nikolaeva von einer zugrunde liegenden Hervorhebung durch den Sprecher aus, das heißt, dass dieser Mechanismus auf Ebene der Pragmatik und weniger der Grammatik beherbergt ist (verglichen Nikolaeva 2003: 5). Den Beschreibungen von Nikolaeva 2003 und Simonenko 2014 gemeinsam ist, dass beide diesen Mechanismus in erster Linie in Anlehnung an ähnliche Verwendungen des definiten Artikels 
in Artikelsprachen beschreiben, das heißt, das Possessivsuffix wird als Marker für die Vorerwähntheit des Targets analysiert. Betrachtet man jedoch die Beispiele dazu, fallen weitere Gemeinsamkeiten auf: Das Target wird im vorangehenden Satz als Subjekt realisiert, und zwar zusammen mit einem weiteren Referenten, die Personalmarkierung am Prädikat ist daher nicht im Singular. Die vorliegende Arbeit geht davon aus, dass es sich beim Subjekt des vorangehenden Satzes daher sowohl um das Target als auch um den zukünftigen Referenzpunkt handelt, an welchem das Target im Folgesatz verankert wird, die Vorerwähnung in der Gruppe und die Weiterführung eines einzelnen Referenten kann demnach als eine Art GanZES TeIL-Verhältnis verstanden werden, was möglicherweise die Verwendung der Possessivkonstruktion in solchen Vorkommen begründet. Solche Belege, in welchen das Prädikat im Dual steht sind meines Erachtens besonders geeignet, diese Annahme zu untermauern.

Da es sich gleichzeitig ausschließlich um die Relation Person Person handelt, zählen zu diesem Mechanismus nahezu alle als GRUPPE EINER bezeichneten Relationen. Simonenko 2014 beschreibt einen derartigen Mechanismus für das Komi und das Mari und erwähnt, dass er für das Chantische nicht belegt werden könne (verglichen Simonenko 2014: 4). In der vorliegenden Arbeit jedoch finden sich für alle drei ob-ugrischen Dialekte Vorkommen dieser Art, und zwar überwiegend sogar im Dual (35 der 53 Belege). Möglicherweise können auch drei Belege des Ungarischen (0040, 0286, 0553), welche der Struktur im Ob-Ugrischen entsprechen, ebenfalls mit zu diesem Mechanismus gezählt werden. Es ist außerdem anzumerken, dass vorwiegend das Possessivsuffix der dritten Person Singular Einbesitz in der Konstruktion erscheint, und die wenigen Belege mit dem Possessivsuffix der dritten Person Plural Einbesitz auf die Vorkommen mit Prädikat im Plural im Satz zuvor entfallen. Wie die Beschreibung der Vorkommen verdeutlicht, müssen hier sowohl die syntaktischen und semantischen Rollen als auch andere reference-tracking-Mechanismen mit in die Analyse einbezogen werden, da es hier nicht alleine um die Vorerwähnung der einzelnen Referenten geht, sondern auch um deren Kodierung. Der Mechanismus der Known Group ist damit ein komplexes Zusammenspiel mehrerer anaphorischer Verweismittel, die Hauptkriterien für diesen Mechanismus sind die folgenden:

- vorwiegend Possessivsuffix 3. Person Singular Einbesitz

- Das Target und der Referenzpunkt werden gemeinsam als Subjekt im Satz zuvor erwähnt

- Das Prädikat des vorangehenden Satzes ist nicht singularisch (und vorwiegend im Dual)

- Der Referenzpunkt wird als Leerstelle in der Konstruktion realisiert

- Die Relation ist Person Person (Gruppe EIner)

Zum derzeitigen Stand der Analyse werden unter diesem Mechanismus sowohl die Dual- als auch Pluralvorkommen gemeinsam geführt, und auch solche Belege, in welchen sich ein weiterer Satz zwischen gemeinsamer Vorerwähnung und Possessivkonstruktion befinden, werden ebenfalls als Known Group klassifiziert, wenn es sich dabei um einen Einschub handelt. Die überwiegende Anzahl der Belege sind wieder aus den ob-ugrischen Sprachen, und vorwiegend wieder aus dem Mansischen und Kazym-Chantischen. Fast in allen Belegen erscheint das Possessivsuffix der dritten 
Person Singular Einbesitz, dem wiederum eine gewisse hinweisende Funktion auf den neuen Referenten in Subjektposition, ähnlich wie beim Topic Shift Mechanismus und dem Turn-Taking attestiert werden kann.

Tatsächlich liefert Nikolaeva 2003 in diesem Zusammenhang ein Beispiel aus dem Udmurtischen, welches der Known Group in der vorliegenden Arbeit ähnelt (Nikolaeva 2003: 11).

\subsubsection{Die Textumgebung der Konstruktion VN-Px}

Wie eingangs erwähnt, beläuft sich die Systematisierung der Konstruktionen mit Possessivsuffixen anhand der Vorerwähntheit des Targets zwangsläufig ausschließlich auf eine Analyse der Konstruktion SuBs-Px. Bezieht man jedoch bei der Konstruktion VN-Px die Textumgebung, das heißt, in diesem Fall die Vorerwähnung und Fortführung des Referenzpunktes, ebenfalls mit in die Analyse ein, zeigen sich wiederkehrende Strukturen, welche in gewisser Hinsicht auch als textstrukturierende Mechanismen mithilfe von Possessivsuffixen bezeichnet werden können. In diesem Zusammenhang werden ausschließlich die Konstruktionen mit Verbalnomina der ob-ugrischen Sprachen berücksichtigt, in ihrer Funktion als Nebensatzäquivalente gehen sie dem jeweiligen Hauptsatz voraus. Diese Konstruktionen lassen sich zunächst im Hinblick auf die Fortführung des Referenzpunktes im folgenden Hauptsatz in etwa gleichmäßig in zwei Hauptgruppen unterteilen: In 73 Belegen erfolgt ein Referentenwechsel, während in 77 Konstruktionen der Referent fortgeführt wird. Die Daten sind nicht ausreichend, um gesicherte Erkenntnisse zu erhalten; es lassen sich jedoch ein paar wiederkehrende Regularitäten feststellen, welche nicht unerwähnt bleiben sollten. So wird zum Beispiel in rund einem Drittel der temporalen Nebensatzkonstruktionen (und zwar ausschließlich gleichzeitig und vorzeitig) eine zuvor beschriebene Handlung nochmals wiederholt, während im zugehörigen Hauptsatz eine neue Handlung beschrieben wird. Dies trifft auch auf die Hälfte der Konverb-Konstruktionen des Mansischen zu, wohingegen im Kazym-Chantischen nur in drei Vorkommen der Konstruktion VN-Px die Handlung wiederholt wird. In manchen Konstruktionen ist ein Demonstrativpronomen enthalten (sowohl substantivische wie, diese,r,s' als auch adverbiale wie ,dorthin'), in den meisten Fällen, in welchen dieses als Determinierer dem Verbalnomen zugeordnet werden kann, handelt es sich um die Nebensatzkonstruktionen, welche eine Wiederholung der Handlung ausdrücken. Im Surgut-Chantischen entspricht dies zwei Drittel der Vorkommen. Insofern kann das Erscheinen der Konstruktion VN-Px zur Wiederholung der vorausgegangenen Handlung als textstrukturierend interpretiert werden, indem ein Handlungsabschnitt abgeschlossen wird, bevor im Hauptsatz ein neuer beginnt.

(80) NM_text_004_0023:3

$\begin{array}{llllll}\text { wina } & -I & \text { aj } & -t & -a w e & -t \\ \text { alcohol } & - \text { INST } & \text { drink } & \text {-CAUS } & \text {-PASS } & -3 P L\end{array}$

'Ihnen wird Branntwein zu trinken gegeben.

(81) NM_text_004_0024

\begin{tabular}{|c|c|c|}
\hline $\begin{array}{l}\text { aj } \\
\text { drink }\end{array}$ & $\begin{array}{l}-t \\
- \text { CAUS }\end{array}$ & $\begin{array}{l}-i m \\
-C V B\end{array}$ \\
\hline
\end{tabular}

'Während sie so tranken, gingen die Hiesigen davon.' 
Das Demonstrativpronomen scheint zur Hervorhebung dieses Wechsels eingesetzt zu werden, und möglicherweise auch das Auftreten des Referenzpunktes als Personalpronomen.

(82) SK_text_003_0072:5

$\begin{array}{llll}q p: t & -b & t^{i} i & j o \beta ə t \\ \text { house } & \text {-DLAT } & \text { this } & \text { come[PST.3SG] }\end{array}$

'She came to a house.'

(83) SK_text_003_0072:6

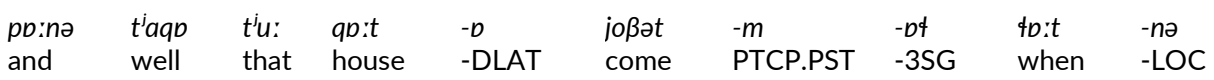

'Indeed, as she came to the house.'

(84) SK_text_003_0072:7

$t^{i} u:$ i:mi jo:star

that old woman say[PRS.3SG]

'The old woman says.'

Ein Referentenwechsel ist dabei nicht nur nicht ausgeschlossen, einige Belege weisen sogar eine Tendenz dahingehend auf, dass die abgeschlossene Handlung von topikalen Referenten begangen wurde und der neue Referent eine geringere topikale Rolle ausübt und dementsprechend kurz in der Handlung erscheint. Das Possessivsuffix hat damit in solchen Fällen ebenfalls eine eher rückweisende Funktion:

(85) NM_Text_004_0060:2

jal- $\quad$ xuj $-a s$

down lie -PST[3SG]

'Er legte sich hin.'

(86) NM_Text_004_0061

xuj -ima te akwmate:rt ak ${ }^{w}$ e:kwa joxt -əs

lie -CVB -3SG once one woman come -PST[3SG]

'Während er dort lag, kam auf ein Mal eine Frau.'

Ein weiteres erwähnenswertes Auftreten der Konstruktion VN-Px kann im SurgutChantischen und möglicherweise auch im Mansischen beobachtet werden: Die Partizipialkonstruktion erscheint im Dual und wiederholt die gemeinsame Handlung von zwei Referenten, während im Hauptsatz einer der beiden Referenten fortgeführt wird.

(87) SK_text_005_0002

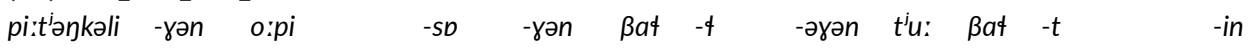
little bird -DU older sister -COLL -DU live -PRS -3DU that live -PTCP.PRS -2DU

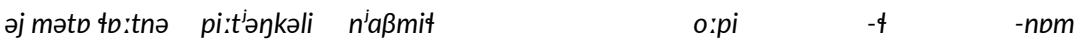
some day little bird begin to speak.PRS[3SG] older sister $\quad-S G<3 S G \quad$-APP

'There lived a little bird and his sister. Living so, at some point the little bird says to his sister.'

Der Mechanismus Repeat ähnelt daher in gewisser Weise dem der Known Group - das Herausgreifen und Fortführen eines Referenten aus einer zuvor erwähnten Gruppe, nur dass das Possessivsuffix hier Bestandteil der Konstruktion ist, welche auf die vorherigen Referenten zurückweist und nicht auf den fortgeführten hinweist. Diese Verwendung der Konstruktion VN-Px tritt im Surgut-Chantischen ausschließlich mit einem Demonstrativpronomen auf. Möglicherweise wird dieser Mechanismus im Mansischen bei Gleichzeitigkeit statt mit einer Partizipial- mit einer Konverb-Konstruktion ausgedrückt, die Belege sind jedoch nicht ausreichend, um darüber eine gesicherte Aussage treffen zu können. 


\subsubsection{Syntaktisch motivierte Mechanismen}

Für das Anchoring ausschlaggebend ist die Vorerwähntheit des Referenzpunktes, sodass die Verankerung des neu erwähnten Targets an einem schon bekannten Referenten überhaupt erfolgen kann. Dieser wird gemeinhin in solchen Fällen in den ugrischen Sprachen als Leerstelle, seltener als Pronomen realisiert. Somit bleiben nur noch solche Konstruktionen mit neu erwähntem Target übrig, in welchen der Referenzpunkt als Substantiv realisiert wird. Hinzu kommen lediglich 135 Belege der rund 1000 Vorkommen von Konstruktionen mit vorerwähnten Targets, welche ein Substantiv als Referenzpunkt aufweisen. Auf diese Art der Konstruktion Su bs Subs-Px wird in der Uralistik mitunter mit dem Begriff »Izafet-Konstruktion« Bezug genommen (zum Beispiel Honti 2005, Honti 2006, Honti 2008 und Honti 2012).

Für die Textstrukturierung sind diese im Gegensatz zum Anchoring von geringerer Bedeutung, stattdessen wird sie durch andere Eigenschaften charakterisiert. Diese Konstruktionen werden in der vorliegenden Arbeit als syntaktisch motivierte Mechanismen beschrieben.

\subsubsection{Izafet im Ungarischen}

Der Begriff »Izafet« (auch »Izāfa«, »Ezāfe«) stammt ursprünglich aus der Arabistik, wurde dann für das Persische übernommen und bezeichnet dort eine Konstruktion, in welcher mithilfe einer Partikel die Zugehörigkeit von Modifizierer und Kopf morphologisch markiert wird. Der Modifizierer kann dabei substantivisch in der Konstruktion auftreten (Honti 2007a: 55). ${ }^{105}$ Die Bedeutung des Wortursprungs ist ,Anschluss oder ,Verbindung' (Honti 2012: 186), die Lesart der Izafet-Konstruktion ist demnach häufig possessiv oder auch eine nähere Bestimmung (zum Beispiel von Adjektiv und Substantiv). Die Izafet-Konstruktion findet sich des Weiteren im Iranischen und in den Turksprachen. Dort jedoch wurde der Begriff aufgrund der arabisch-persisch geprägten Grammatiktradition hin übernommen und nicht aufgrund einer strukturellen Ähnlichkeit. Der Begriff »Ezāfe« bezeichnet demnach eine Konstruktion des Iranischen und Persischen, in welcher "das zweite Wort das erste näher bestimmt « (Honti 2012: 186), »Izafet« hingegen die Possessivkonstruktion des Türkischen, welche head-final ist. Letztere wird als Kontakteinfluss für die Izafet-Konstruktionen in den uralischen Sprachen herangezogen (Honti 2012: 187). Im Zusammenhang mit der Uralistik wurde der Begriff »Izafet« insbesondere von Gábor Bereczki und László Honti verwendet, wobei sich die einzelnen Definitionen hier unterscheiden. Honti beispielsweise rechnet in seiner Terminologie des "Izafet « außer unmarkierten Juxtapositionen auch Konstruktionen mit dependent-marking nicht dazu (Honti 2007a: 56). Für die vorliegende Arbeit wird der Begriff »Izafet« für die Konstruktionen in Anlehnung an Honti 2012 verwendet:

105 Mit der Definition des Izafet sowohl in der Altaistik als auch der Uralistik befasst sich detailliert Honti 2012, die vorliegende Arbeit beschränkt sich auf eine kurze Zusammenfassung und Erläuterung der Terminologie. 
- Possessivsuffix 3. Person Singular/Plural Ein- und Mehrbesitz

- Der Referenzpunkt wird als Substantiv in der Konstruktion realisiert

(88) HU_text_003_0007

\begin{tabular}{|c|c|c|c|c|c|c|c|c|c|}
\hline $\begin{array}{ll}a & p r \\
\text { the } & \text { pr }\end{array}$ & $\begin{array}{l}\text { gram } \\
\text { gram }\end{array}$ & $\begin{array}{l}\text { eret } \\
\text { ame }\end{array}$ & $\begin{array}{l}-e ́ \\
-S G<3 S G\end{array}$ & $\begin{array}{l}\text {-ben } \\
\text {-INE }\end{array}$ & $\begin{array}{l}\text { fejleszt } \\
\text { to educa }\end{array}$ & & $\begin{array}{l}\text {-ett } \\
\text { PTCP.PST }\end{array}$ & $\begin{array}{l}\text { készsé } \\
\text { proficiency }\end{array}$ & $\begin{array}{l}-e k \\
-P L\end{array}$ \\
\hline segítség & -é & $-v e l$ & könny & $-e b b$ & -en & & & & \\
\hline upport & $-S G<3 S G$ & $-\mathrm{COM}$ & easy & $-\mathrm{CMPR}$ & $-M O D$ & & & & \\
\hline lál & -hat & -0 & $-n a k$ & áll & -ás & $-t$ & & & \\
\hline to find & -POSBL & -PRS & $-3 P L$ & to stand & -NMLZ & $-A C C$ & & & \\
\hline
\end{tabular}

'Im Rahmen des Programms erworbene Kompetenzen verbessern auch die Beschäftigungsfähigkeit.'

In den Izafet-Konstruktionen reicht die referentielle Verweiskraft des Possessivsuffixes lediglich zurück bis zum als Substantiv realisierten Modifizierer, dieser dient als Zusatzinformation zum Kopf. Der Referenzpunkt in Funktion von Zusatzinformation wird mithilfe des Possessivsuffixes 3. Person Singular als zugehörig zum Target markiert. Somit kann hier mehr von einem grammatischen Marker vergleichbar mit dem Genitiv-Marker als von einem referentiellen Personalmarker gesprochen werden. Mit dem Possessivsuffix wird ebenfalls eine Relation zwischen Kopf und Modifizierer angezeigt, jedoch wird diese durch persönliche Referenz angezeigt, was das Possessivsuffix zunächst von einer Kasusmarkierung unterscheidet. Ein Kasus kongruiert im Numerus, das Possessivsuffix kongruiert mit dem Possessum im Numerus, mit dem Besitzer in Person und Numerus. Für die Annahme, dass es sich bei der Izafet-Konstruktion eher um eine grammatische Verbindung denn Referentialität handelt, spricht ebenfalls, dass es sich bei den semantischen Relationen, welche in den Izafet-Konstruktionen ausgedrückt werden, fast ausschließlich um GanZES TEIL-Relationen handelt oder um solche, die den Begriff AвSTRAKт enthalten (hier handelt es sich um 76 der 89 in Kapitel 5.1.1.4 erwähnten Konstruktionen, welche dem Mechanismus Izafet zugeordnet werden können). Eine mögliche Grammatikalisierung des Possessivsuffixes der dritten Person Singular hin zu einem Marker grammatischer Dependenz ähnlich dem Genitiv scheint daher im Ungarischen durchaus möglich. Hierfür spricht auch die an sich fehlende Kongruenz in den Konstruktionen: Den grammatischen Beschreibungen des Ungarischen ist zu entnehmen, dass die Possessivsuffixe der dritten Person Plural Ein- und Mehrbesitz nur erscheinen, wenn der Referenzpunkt als Leerstelle oder Pronomen realisiert wird. Bei einem Substantiv wird das jeweilige Possessivsuffix im Singular verwendet (verglichen Kapitel 1.2.1 Anmerkungen zu den Paradigmen). Möglicherweise ist dies ein weiterer Hinweis auf die Grammatikalisierung, wenn der Referenzpunkt offensichtlich nicht (mehr) im Numerus kodiert wird. Formen des Mehrbesitzes (das heißt das Target im Plural) sind hingegen vorhanden, wenn auch in weitaus geringerer Anzahl. Insgesamt werden 278 Konstruktionen zum Izafet gezählt.

\subsubsection{Kollokation im Ob-Ugrischen}

Von den ugrischen Sprachen wird im Allgemeinen nur dem Ungarischen die Izafet-Konstruktion attestiert, in älteren Quellen, zum Beispiel Simonyi $1914^{106}$ finden 
sich jedoch auch Verweise auf das Izafet im Ob-Ugrischen (Honti 2007a: 56). Auch im Korpus der vorliegenden Arbeit findet sich eine Gruppe von Belegen aus dem Ob-Ugrischen, welche den Kriterien für das Izafet entsprechen, nämlich die Konstruktionsart Subs Subs-Px.

Besonders hervorzuheben sind auch hier Belege, in welchen der als Substantiv realisierte Referenzpunkt in der Konstruktion zum ersten Mal erwähnt wird: Dies ist an sich ein Widerspruch zur allgemeinen Beschreibung des Possessivsuffixes im Ob-Ugrischen (dies ist jedoch in 25 der 105 Belege der Fall). Dort wird davon ausgegangen, dass erstens nur topikale, das heißt vorerwähnte Referenten mit Possessivsuffixen, kodiert werden können und zweitens die unmarkierte Juxtaposition (SUBs SUbS) weit häufiger als die Possessivkonstruktion generell (verglichen Kapitel 5.1.1) sowie eine Konstruktion aus Subs Subs-Px im Besonderen ist (Honti 2012 191). Erwähnenswert ist des Weiteren, dass es sich besonders häufig um solche Fälle handelt, in welchen die Wortart des Kopfes der Konstruktion nicht eindeutig bestimmbar ist oder der Kopf sowohl als Substantiv als auch als Postposition bestimmt werden kann, und dass zwischen Referenzpunkt und Target die Relation Ganzes Teil besteht (zum Beispiel Inneres/Innen, Mitte; 71 der 105 Belege, verglichen Kapitel 5.1.3).

(89) NM_text_002_0018:1

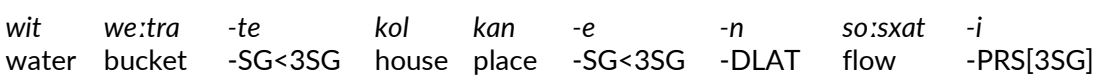

'Das Wasser rann aus dem Eimer auf den Boden.'

In diesen Fällen kann entweder davon ausgegangen werden, dass sich auch im ObUgrischen entweder eine Tendenz zu Izafet-artigen Konstruktionen belegen lässt, weist man dem Kopf die Wortart Substantiv zu - oder es handelt sich um eine obligatorische Realisierung, wie man bei den Postpositionen generell beobachten kann, und man nimmt für den Kopf als Wortart die der Postposition an. In beiden Fällen ist jedoch eine Tendenz weg vom referentiellen Personalmarker hin zu einem Marker von grammatischem Agreement ähnlich dem Izafet zu beobachten. Solche Konstruktionen des Ob-Ugrischen werden in der vorliegenden Arbeit unter dem Begriff »Kollokation« zusammengefasst. Unter »Kollokation « versteht man »charakteristische, häufig auftretende Wortverbindungen, deren gemeinsames Vorkommen auf einer Regelhaftigkeit gegenseitiger Erwartbarkeit beruht, also primär semantisch (nicht grammatisch) begründet ist « (Bußmann 2002: 353). Dies ist insbesondere durch die Ganzes TeiLRelation der Konstruktionen gegeben. Die Kriterien der Kollokation sind wie folgt zusammengefasst:

- Possessivsuffix der 3. Person Singular/Dual/Plural Ein-, Dual- und Mehrbesitz

- Der Referenzpunkt erscheint als Substantiv in der Konstruktion

- Die semantische Relation ist vorwiegend GanZes TeIL

Die Analyse der Possessivkonstruktionen in diesem Teilkapitel war darauf ausgelegt, eine alternative Betrachtung der Vorkommen von Possessivsuffixen zu demonstrieren, welche unabhängig von gängigen Aussagen insbesondere über Possessivkonstruktionen erfolgen sollte. Dies war zum einen die Zweiteilung auf possessive und nicht possessive Verwendungen, die Nicht-Berücksichtigung des Referenzpunktes (und damit 
einhergehende Reduzierung des Possessivsuffixes zu einem nicht-referentiellen Marker) und die Annahme ausschließlich neuer Targets ohne Fortführung im weiteren Handlungsverlauf. Aus diesem Grund hat sich eine Berücksichtigung der Textumgebung besonders geeignet. Mit deren Hinzunahme konnten wiederkehrende Strukturen in der Verwendung von Konstruktionen mit Possessivsuffixen aufgezeigt werden, welche für eine eingehendere Betrachtung der einzelnen Possessivkonstruktionen als bisher sprechen. Die Analyse der Konstruktion VN-Px am Rande hat überdies gezeigt, dass auch andere Konstruktionen mit Possessivsuffixen in Zukunft eingehender betrachtet werden sollten.

\subsubsection{Semantisch motivierte Mechanismen}

Die Berücksichtigung der Textumgebung ist jedoch nur eine Möglichkeit einer eingehenderen Betrachtung. Sie ist im Hinblick auf die Datengrundlage der vorliegenden Arbeit geeignet, würde aber Verwendungen, welche über die Text-Deixis hinausgehen möglicherweise auch nicht umfassend beschreiben können. Auf der anderen Seite kann durch eine Analyse, welche den Schwerpunkt auf andere Aspekte der Konstruktionen mit Possessivsuffixen setzt, eine andere Systematisierung erfolgen. Dies ist zum Beispiel der Fall, wenn man die Semantik, das heißt die in Kapitel 5.1.1.4 beschriebenen Relationen zwischen Referenzpunkt und Target der Textumgebung, vorzieht.

Dabei rückt zum Beispiel die Relation Person LoKalisation noch einmal in den Fokus: So lässt sich eine Gruppe von 81 Belegen zusammenstellen, welche vorwiegend vorerwähnte Targets beinhaltet und darüber hinaus einige der Vorkommen, welche bisher nicht eindeutig zugeordnet werden konnten. Mit der gemeinsamen Bewegung oder Existenz an einem Ort, mit welchem bislang jedoch nur einer der Referenzpunkte im Zusammenhang stand, können diese Vorkommen erklärt und zusammengefasst werden. Der Mechanismus Location kann daher wie folgt kategorisiert werden:

- Die Relation ist Person Lokalisation

- Der Referenzpunkt ist gleichzeitig Subjekt und Agens (seltener Patiens)

- Der Referenzpunkt ist vorerwähnt und wird vorwiegend als Leerstelle realisiert

- Das Target fungiert als lokaladverbiale Ergänzung im Satz

(90) NM_text_012_0227:1

\begin{tabular}{|c|c|c|c|c|c|c|}
\hline $\begin{array}{l}\text { punk } \\
\text { head }\end{array}$ & $\begin{array}{l}-u p \\
\text {-ADJZR }\end{array}$ & $\begin{array}{l}\text { me:yk }{ }^{w} \\
\text { forest spirit }\end{array}$ & & $\begin{array}{l}\text { ojka } \\
\text { old man }\end{array}$ & $\begin{array}{l}\text { joxt } \\
\text { arrive }\end{array}$ & $\begin{array}{l}-m \\
\text {-PTCP.PST }\end{array}$ \\
\hline $\begin{array}{l}-t e \\
-S G<3 S G\end{array}$ & $\begin{array}{l}-n \\
- \text { DLAT }\end{array}$ & $\begin{array}{l}\text { joxt } \\
\text { arrive }\end{array}$ & $\begin{array}{l}-e s \\
-P S T\end{array}$ & $\begin{array}{l}-\partial t \\
-3 P L\end{array}$ & & \\
\hline
\end{tabular}

'They arrived to the land where they met the five-headed forest spirit old man.'

Eine Korrelation mit dem Konstruktionstyp ReLSatz Subs-Px wie in Beispiel (90) kann aufgrund der geringen Anzahl der Belege nicht verlässlich eingeschätzt werden. Diese Kategorisierung erfolgt zusätzlich zur Kategorisierung nach den oben vorgestellten Mechanismen, das heißt, mitunter trägt ein Vorkommen beide Bezeichnungen, zum Beispiel Anchoring und Location oder Topic Chain und Location. Auffallend ist jedoch, dass keine Kombination von Izafet und Location auftritt, was meines 
Erachtens ein weiteres Anzeichen für die Funktion des Possessivsuffixes als grammatischer Marker in dieser Konstruktion ist: Der Referenzpunkt in der Izafet-Konstruktion übt nicht die Rolle des Subjekts im Satz aus und ist folglich kein Referent mit hoher topikaler Rolle.

Dem Großteil der Belege konnte durch die hier vorgestellten Analyseansätze ein Mechanismus zugeordnet werden, für welchen die Grundfunktion des Possessivsuffixes als Marker einer nicht näher bestimmten Relation eingesetzt werden kann und weit über die allgemein vorherrschende Annahme des Ausdrucks von Possessivität hinausgeht. Die Mechanismen lassen sich überdies in drei Gruppen einordnen, welche als pragmatisch, syntaktisch und semantisch motivierte Mechanismen bezeichnet wurden. Die pragmatisch motivierten Mechanismen sind diejenigen, welche nicht obligatorisch sind und je nach Intention des Sprechers zur Textstrukturierung eingesetzt werden können. Die syntaktisch motivierten Mechanismen sind diejenigen, in welchen das Possessivsuffix obligatorisch die Zugehörigkeit von Kopf und Modifizierer markiert. Hierunter fallen nicht nur die Izafet-Konstruktionen, sondern auch solche, in denen das Possessivsuffix mit dem Modifizierer in Person und Numerus kongruiert, zum Beispiel die Konstruktion PostP-Px der ob-ugrischen Sprachen. Auch einige Vorkommen der Konstruktion VN-Px (zum Beispiel Konstruktionen mit Verben der Wahrnehmung) könnten mit zu dieser Gruppe gerechnet werden sowie eventuell die Relativsatz-Konstruktionen. Für letztere sind jedoch nicht genügend Belege vorhanden, um verlässliche Aussagen treffen zu können.

Zwischen beiden Mechanismen, den pragmatisch und den syntaktisch motivierten, ergibt sich meines Erachtens insbesondere ein Unterschied zwischen Konstruktionen mit Leerstelle und Substantiv als Referenzpunkt - sie bilden in gewisser Hinsicht die Gegenpole der referentiellen Verweiskraft und grammatischen Markierung mit dem Possessivsuffix. Abgeleitet auf die Textstrukturierung ist insbesondere bei neu erwähnten Targets der Kontrast zwischen dem Anchoring und dem Izafet hervorzuheben. Beide Konstruktionsarten wurden sowohl im Zusammenhang mit den Possessivkonstruktionen der uralischen Sprachen als auch sprachübergreifend bereits in der Fachliteratur erwähnt und konnten für die ugrischen Sprachen belegt werden. Es konnte außerdem belegt werden, dass die Konstruktionen mit neu erwähntem Target nicht den Großteil der Possessivkonstruktionen ausmachen, der größte Teil ist vorerwähnt. Das Anchoring ist demnach nicht die primäre Funktion der Possessivkonstruktion in den ugrischen Sprachen und sie entspricht auch nicht der einzigen Konstruktionsart für neu erwähnte Targets.

Ein semantisch motivierter Mechanismus wäre beispielsweise die als Location bezeichnete Verwendung, da hier die semantischen Rollen von Referenzpunkt und Target möglicherweise das Auftreten des Possessivsuffixes erklären. Auch eine Kategorisierung von Possessivsuffixen nach der Relation von Agens und Patiens wäre denkbar - für die Korpusbelege der vorliegenden Arbeit liefert diese Gruppierung jedoch keine nennenswerten weiteren Erkenntnisse. Sollte die Possessivsuffigierung von Verwandtschaftstermini in manchen Dialekten obligatorisch sein (verglichen Filchenko 2007), so wäre auch dies ein semantisch motivierter Mechanismus. Die folgende Übersicht systematisiert noch einmal die Konstruktionen je nach Art des Mechanismus: 


\begin{tabular}{|c|c|c|c|}
\hline Konstruktion & Mechanismus & Vorkommen & Motivation \\
\hline \multirow{6}{*}{ SuBs-Px } & Anchoring & \multirow{4}{*}{$\begin{array}{l}\text { Ungarisch } \\
\text { Ob-Ugrisch }\end{array}$} & \multirow{7}{*}{ pragmatisch } \\
\hline & Topic Chain & & \\
\hline & Topic Shift & & \\
\hline & Anrede & & \\
\hline & Turn-Taking & \multirow{3}{*}{ Ob-Ugrisch } & \\
\hline & Known Group & & \\
\hline VN-Px & Repeat & & \\
\hline \multirow{4}{*}{ SuBs-Px } & Izafet & \multirow{3}{*}{ Ungarisch } & \multirow{7}{*}{ syntaktisch } \\
\hline & Habeo-Konstruktion & & \\
\hline & Nezessiv-Konstruktion & & \\
\hline & Kollokation & Ob-Ugrisch & \\
\hline \multirow{2}{*}{ VN-Px } & Modalverb-Konstruktion & Ungarisch & \\
\hline & Verben der Wahrnehmung & \multirow{2}{*}{ Ob-Ugrisch } & \\
\hline PostP-Px & PostP-Px & & \\
\hline SuBs-Px & Location & $\begin{array}{l}\text { Ungarisch } \\
\text { Ob-Ugrisch }\end{array}$ & semantisch \\
\hline
\end{tabular}

Tabelle 24: Zusammenführung von Konstruktionen und Mechanismen.

Die in diesem Teilkapitel vorgestellte Analyse hat nicht alle Belege der vorliegenden Arbeit berücksichtigen können. Manches wurde daher von vornherein aus der Analyse ausgeschlossen. Sätze aus der Feldforschung beispielsweise, in welchen ein Referent aus dem Stimulus im elizitierten Satz als Target realisiert wird, konnten nicht mit Zuhilfenahme einer Textumgebung beschrieben werden, sie wurden daher nur am Rande erwähnt. Auch eine weitere Gruppe von Belegen, welchen gemeinsam ist, dass Vorerwähnung und Referenzpunkt sich nicht eins zu eins entsprechen, wurde nicht weiter analysiert. Dies bedeutet, dass zum Beispiel der Referenzpunkt zuvor Teil einer Gruppe von zwei oder mehreren Referenten war und nun einzeln als Referenzpunkt fungiert. Umgekehrt können zwei Referenten einzeln vorerwähnt worden sein und nun gemeinsam als Referenzpunkt fungieren.

Angesichts der Tatsache, dass es sich um ein vorerwähntes Target handelt und der Referenzpunkt, welchem das Target zugeordnet wird, in gewisser Weise wechselt, wäre dies eine mögliche Erklärung für das Vorkommen der Possessivkonstruktion. Ähnliches ist anzunehmen, wenn ein Referenzpunkt mit einem vorerwähnten Target neu in der Possessivkonstruktion eingeführt wird. Da es sich hier um eine relativ geringe Anzahl an Belegen handelt, konnten keine weiteren Aussagen zum derzeitigen Stand der Analyse getroffen werden.

Es bleibt noch auf eine weitere Art der Vorerwähnung des Targets zu verweisen, der indirekten Vorerwähnung, zum Beispiel wenn es sich um die Nominalisierung 
einer vorangegangenen Handlung handelt. Die Gruppe von 127 Belegen, auf welche die indirekte Vorerwähnung des Targets zutrifft, ist jedoch zu heterogen, um eine gesicherte Aussage über etwaige Auswirkungen der indirekten Vorerwähnung treffen zu können. Die Belege wurden daher in der Kategorisierung nicht weiter berücksichtigt. 


\section{Schluss}

Thema der vorliegenden Arbeit sind die Funktionen von Possessivsuffixen in den ugrischen Sprachen. Diese wurden anhand von Vorkommen von Konstruktionen mit Possessivsuffixen in einem rund 2.800 Belege umfassenden Korpus beschrieben, anhand der Konstruktionsarten gruppiert und mit Zuhilfenahme der Textumgebung systematisiert. Die Beschreibung erfolgte unter Berücksichtigung theoretischer Ansätze aus der Kognitiven Linguistik (insbesondere der Referenzpunkt-Konstruktionen) sowie aus der Functional Grammar (insbesondere der Text- bzw. Referenzlinguistik). Ziel der deskriptiven Analyse war nicht nur, die bisherigen Forschungsansätze zu Possessivkonstruktionen und Possessivsuffixen im Allgemeinen und in den ugrischen Sprachen im speziellen mit den Vorkommen im eigenen Korpus zu vergleichen. Darüber hinaus sollte die Analyse der vorliegenden Arbeit durch ihren semasiologischen Ansatz solche Parameter heranziehen, welche bislang eher unberücksichtigt waren, und gleichzeitig Aspekte, welche bislang eher über-evaluiert wurden, außen vor lassen. Schwerpunkte bildeten demnach der im Possessivsuffix kodierte Referenzpunkt und dessen Realisierung in der Konstruktion, die Vorerwähntheit des Targets in der Possessivkonstruktion im Besonderen und die Textumgebung der Konstruktionen mit Possessivsuffixen im Allgemeinen. Die Unterteilung in possessive und nicht-possessive Verwendungen sowie der Definitheits-Ansatz wurden nicht für die Analyse herangezogen.

Die Paradigmen der Possessivsuffixe lassen sich ebenfalls nach Konstruktionen einteilen. Hierbei ist anzumerken, dass in der Konstruktion Subs-Px das Possessivsuffix zwei Referenten kodiert. In allen anderen Konstruktionen wird lediglich ein Referent kodiert, sodass sich die jeweiligen Paradigmen hier unterscheiden: Die Spalten für den Mehrbesitz entfallen. Im Folgenden werden die Tabellen aus Kapitel 1.2 für die vier behandelten Dialekte noch einmal aufgeführt. Die Bezeichnungen Possessor und Possessum wurden dabei entfernt (respektive in der Tabelle durchgestrichen), dafür wurden alle drei Konstruktionen berücksichtigt.

Die Formen des Einbesitzes in der Konstruktion Subs-Px sind an und für sich formgleich mit den Formen der Konstruktionen VN-Px und PostP-Px, da der Numerus des Targets im Singular nicht wie bei der objektiven Konjugation mit einem eigenen Ko-Affix markiert wird (verglichen Rédei 1965: 44). Kleinere Unterschiede zwischen den Konstruktionen sind jedoch erkennbar. Im Surgut-Chantischen beispielsweise verändert sich der Bindevokal der Possessivsuffixe im Singular (BV bei SuBs-PX -ə-, bei VN-Px $-\boldsymbol{P}-$-). Die Konstruktionen VN-Px des Surgut-Chantischen weisen in etwa zu gleichen Teilen in der zweiten Person Singular die Endung $-\boldsymbol{e} n$ und $-\boldsymbol{B}$ auf. Ein Unterschied zwischen der Personalmarkierung der zweiten Person Singular kommt auch im Paradigma Subs-Px des Surgut-Chantischen vor: Hier hat der Einbesitz die Personalendung -(a)n, der Dual- und Mehrbesitz die Personalendung - $\boldsymbol{p}$. Möglicherweise besteht hier ein Zusammenhang. Ob auch der Bindevokal der zweiten und dritten Person Dual Subs-Px gegenüber der Konstruktion PозтP-Px im Kazym-Chantischen paradigmatisch wechselt oder ob weitere derartige Unterschiede existieren, ist anhand der begrenzten Datenmenge nicht endgültig festzustellen. 


\begin{tabular}{|c|c|c|c|c|c|c|}
\hline \multicolumn{2}{|c|}{ Possessof } & \multicolumn{5}{|c|}{ Possessum } \\
\hline \multicolumn{2}{|c|}{ RefP } & \multicolumn{5}{|l|}{ Kopf } \\
\hline & & \multirow[t]{3}{*}{ VN } & \multirow[t]{3}{*}{ PostP } & \multicolumn{3}{|l|}{ SUBS } \\
\hline & & & & \multicolumn{3}{|l|}{ Target } \\
\hline & & & & Singular & Dual & Plural \\
\hline & 1. & $-m$ & $-m$ & $-m$ & -(j)aуәm & -(j)anəm \\
\hline \multirow[t]{3}{*}{ SG } & 2. & $-n$ & $-n$ & $-n$ & -ayən & -(j)an[ən] \\
\hline & 3. & $-(t) e$ & $-e$ & $-(t) e$ & -(j)aye & -(j)ane \\
\hline & 1. & - men & -amen & $-m e n$ & -yamen & -anamen \\
\hline \multirow[t]{3}{*}{ DU } & 2. & $-(e) n$ & $-(e) n$ & $-(e) n$ & -(j)ayen & -nen \\
\hline & 3. & $-(t) e n$ & -ten & $-(t) e n$ & -ayen & -(j)anen \\
\hline & 1. & $-u w$ & $-u w$ & -(j)uw & -ayuw & -anuw \\
\hline \multirow[t]{2}{*}{ PL } & 2. & $-(e) n$ & $-(e) n$ & $-(e) n$ & -(j)ayen & -anən \\
\hline & 3. & -anol & -anal & -(j)anəl & -(j)ayanəl & -an[an]əl \\
\hline
\end{tabular}

Tabelle 25: Paradigma der Possessivsuffixe des Nord-Mansischen.

\begin{tabular}{|c|c|c|c|c|c|c|}
\hline \multirow{2}{*}{\multicolumn{2}{|c|}{$\begin{array}{l}\text { REFP } \\
\text { P }\end{array}$}} & \multirow{2}{*}{\multicolumn{5}{|c|}{$\begin{array}{l}\text { Kopf } \\
\text { Pom }\end{array}$}} \\
\hline & & & & & & \\
\hline & & \multirow[t]{3}{*}{ VN } & \multirow[t]{3}{*}{ PostP } & \multicolumn{3}{|l|}{ SuBS } \\
\hline & & & & \multicolumn{3}{|l|}{ Target } \\
\hline & & & & Singular & Dual & Plural \\
\hline & 1. & $-m$ & $-m$ & $-m$ & -nəłam & -tam \\
\hline \multirow[t]{3}{*}{ SG } & 2. & $-n$ & $-n$ & $-n$ & -(j)nətan & -tan \\
\hline & 3. & -4 & -4 & -4 & $-\eta ә t$ & $-t a t$ \\
\hline & 1. & -દmən & -Emən & $-\varepsilon m ə n$ & -(j)nəłmən & -łəmən \\
\hline \multirow[t]{3}{*}{ DU } & 2. & -ən & $-n$ & $-ə n$ & -(j) நəłən & -łən \\
\hline & 3. & -ən & $-n$ & $-ə n$ & -(j)クəなən & -łən \\
\hline & 1. & $-\beta$ & $-\beta$ & $-\beta$ & $-(j) \eta \partial \Varangle \partial \beta$ & $-t a \beta$ \\
\hline \multirow[t]{2}{*}{ PL } & 2. & $-n$ & $-n$ & $-n$ & -(j)yəłən & -tan \\
\hline & 3. & -4 & -4 & -4 & - nətat & -tat \\
\hline
\end{tabular}

Tabelle 26: Paradigma der Possessivsuffixe des Nord-Chantischen (am Beispiel des Kazym-Dialektes). 


\begin{tabular}{|c|c|c|c|c|c|c|}
\hline \multicolumn{2}{|c|}{ Possessor } & \multicolumn{5}{|c|}{ Possessum } \\
\hline \multicolumn{2}{|l|}{ RefP } & \multicolumn{5}{|l|}{ Kopf } \\
\hline & & \multirow[t]{3}{*}{ VN } & \multirow[t]{3}{*}{ PostP } & \multicolumn{3}{|l|}{ SuBS } \\
\hline & & & & \multicolumn{3}{|l|}{ Target } \\
\hline & & & & Singular & Dual & Plural \\
\hline & 1. & $-e m$ & $-ә m$ & $-ə m$ & -yəłem & -tem \\
\hline \multirow[t]{3}{*}{ SG } & 2. & $-e n /-e$ & -ən & $-(\partial) n$ & - rate & $-4 e$ \\
\hline & 3. & -ed & $-\partial f$ & $-\partial \phi$ & $-\gamma \partial \phi$ & -ted \\
\hline & 1. & -imən & -imən & -imən & - уәぬәтәn & -ðәтәn \\
\hline \multirow[t]{3}{*}{ DU } & 2. & -in & -in & -in & -yəłən & $-4 ә n$ \\
\hline & 3. & - in & -in & $-(j i) n$ & -yəłən & $-\Varangle ә n$ \\
\hline & 1. & $-i \beta$ & $-i \beta$ & $-B \vee \beta$ & $-\gamma ә \nrightarrow \partial \beta$ & $-\Varangle \partial \beta$ \\
\hline \multirow[t]{2}{*}{ PL } & 2. & - in & -in & -in & -yəłən & $-\Varangle ә n$ \\
\hline & 3. & -if & -if & $-B V 4$ & $-\gamma \partial t$ & -tet \\
\hline
\end{tabular}

Tabelle 27: Paradigma der Possessivsuffixe des Ost-Chantischen (am Beispiel des Surgut-Dialektes).

\begin{tabular}{|c|c|c|c|c|c|}
\hline \multicolumn{2}{|c|}{ Possessor } & \multicolumn{4}{|c|}{ Possesstm } \\
\hline \multicolumn{2}{|c|}{ RefP } & \multicolumn{4}{|l|}{ Kopf } \\
\hline & & \multirow[t]{3}{*}{ VN } & \multirow[t]{3}{*}{ PostP } & \multicolumn{2}{|l|}{ SuBS } \\
\hline & & & & \multicolumn{2}{|l|}{ Target } \\
\hline & & & & Singular & Plural \\
\hline & 1. & -em/-om & $-e m /-a m$ & $-B V m$ & $-i m$ \\
\hline \multirow[t]{3}{*}{ SG } & 2. & -ed & -ed & $-B V d$ & $-i d$ \\
\hline & 3. & $-(j) a /-(j) e$ & $-(j) a /-(j) e$ & $-(j) a /-(j) e$ & $-i$ \\
\hline & 1. & $-n k$ & $-n k$ & $-n k$ & -ink \\
\hline \multirow[t]{2}{*}{ PL } & 2. & -tok & -tok & -tok & -itok \\
\hline & 3. & $-k$ & $-k$ & $-k$ & $-i k$ \\
\hline
\end{tabular}

Tabelle 28: Paradigma der Possessivsuffixe des Ungarischen.

Die Berücksichtigung der Textumgebung konnte gewisse wiederkehrende Strukturen bei der Verwendung von bestimmten Konstruktionen mit Possessivsuffixen verdeutlichen sowie einige unterschiedliche Tendenzen bei der Auswirkung bestimmter Konstruktionen auf die Textstrukturierung einerseits und der Verwendung dieser zwischen dem Ungarischen und den ob-ugrischen Sprachen andererseits aufzeigen. So steht insbesondere 
die Verankerung eines Referenten an einem Referenzpunkt der Markierung der Zugehörigkeit von Kopf und Modifizierer innerhalb der Konstruktion gegenüber. Neben dem Anchoring an sich, welches ein neu erwähntes Target durch die Salienz des Referenzpunktes für den Hörer zugänglicher macht, sind hier auch hinweisende Faktoren des Possessivsuffixes auf das Taget in einigen Konstruktionen mit vorerwähntem Target zu erwähnen (zum Beispiel Turn-Taking, Topic Shift). Ihnen gegenüber steht beispielsweise die Izafet-Konstruktion. Letztere ist vorwiegend eine Erscheinung des Ungarischen, während Turn-Taking und Topic Shift vorwiegend in den ob-ugrischen Sprachen auftreten. Hier ist auch nochmals eine leichte Abweichung zwischen dem Surgut-Chantischen und den Nord-Dialekten des Chantischen und Mansischen zu beobachten.

\begin{tabular}{lcccc}
\hline Mechanismus & HU & NM & KK & SK \\
\hline Topic Chain & 197 & 303 & 142 & 96 \\
\hline Anchoring & 272 & 152 & 101 & 58 \\
\hline Izafet & 278 & & & \\
\hline Kollokation & 31 & 107 & 29 & 8 \\
\hline Topic Shift & 3 & 19 & 25 & 30 \\
\hline Known Group & 2 & 10 & 15 & 6 \\
\hline Turn-Taking & & & 1 \\
\hline
\end{tabular}

Tabelle 29: Verteilung der Mechanismen auf die einzelnen Sprachen bzw. Dialekte.

Die Realisierung des Referenzpunktes hat somit je nach Konstruktionsart unterschiedliche Auswirkungen. Die Realisierung als Leerstelle spricht von Topik-Kontinuität und hoher Salienz des kodierten Referenten. Sie machen die mit Abstand häufigste Realisierung des Referenzpunktes aus. Als zweithäufigste Realisierung folgt nicht das Personalpronomen, sondern das Subjekt. Somit hängt die Verwendung eines Personalpronomens weniger mit der Topik-Kontinuität oder Salienz des Referenzpunktes zusammen, sondern dient eher der Fokussierung dessen, worauf auch das Vorhandensein von emphatischen Personalpronomen hinweist (verglichen auch Kulonen 2007: 195). Nicht jedoch in der Konstruktion PostP-Px im Ob-Ugrischen: Hier scheinen die Personalpronomen ein obligatorischer Bestandteil, das Possessivsuffix demnach ein Marker der Zugehörigkeit zum Kopf der Konstruktion ähnlich den Izafet-Konstruktionen mit Substantiv als Referenzpunkt. Emphatische Personalpronomen kommen hier in den Korpusbelegen nicht vor. Substantive dienen in seltenen Fällen ebenfalls der Fokussierung des Referenzpunktes (insbesondere Eigennamen) oder weisen im Großteil der Vorkommen auf grammatisches Agreement hin. Ersteres ist eine fakultative Realisierung, letzteres scheint obligatorisch.

Tabelle 30 gibt eine grobe Einschätzung wieder, welche jedoch aufgrund der Datenlage nicht vollständig belegt werden kann. Insbesondere der Bereich der Konstruktion VN-Px bedarf weiterer Daten zur endgültigen Bestimmung. Möglicherweise lässt sich dann dort eine weitere Unterteilung feststellen - je nachdem, ob es sich um einen pragmatisch motivierten Mechanismus handelt, zum Beispiel um eine adverbiale 


\begin{tabular}{lll}
\hline Realisierung RefP & zeigt an & Konstruktion \\
\hline$\varnothing$ & Topik-Kontinuität & SuBS-Px \\
\hline PERSPR & Fokus & VN-Px \\
\cline { 1 - 2 } SUBS & gram Agreement/Fokus \\
\hline Relativsatz & gram Agreement? \\
\hline PERSPR & Topik-Kontinuität \\
\hline SUBS & $?$ \\
\hline$\varnothing$ & $?$ \\
\hline PERSPR & Topik-Kontinuität \\
\hline
\end{tabular}

Tabelle 30: Auswirkung der Realisierung des Referenzpunktes nach Konstruktion.

Nebensatzkonstruktion zur Strukturierung der Handlung (Repeat) oder um einen syntaktisch motivierten Mechanismus, wie die Modalverb-Konstruktion im Ungarischen oder die Konstruktion mit Verben der Wahrnehmung oder den Mirativ/Evidentialis im Ob-Ugrischen. Eventuell ist mit Zuhilfenahme der Realisierung des Referenzpunktes hier auch keine weitere Erkenntnis zu gewinnen, was mitunter damit zusammenhängen könnte, dass die hier als syntaktisch motiviert bezeichneten Mechanismen eine Grammatikalisierung zu finiten Verbalformen im Hauptsatz darstellen anstatt von Nebensatzkonstruktionen. So ließe sich eine vierte Gruppe der Verwendung von Possessivsuffixen anordnen, bei welcher weniger von text-strukturierenden Mechanismen und eher von grammatikalisierten Konstruktionen gesprochen werden kann.

Die Frage nach dem Grad der Grammatikalisierung selbst bleibt ebenfalls noch zu beantworten. Die vorliegende Arbeit geht wie viele andere Abhandlungen (zum Beispiel Fraurud 2001, Gerland 2014a) nicht von einer Grammatikalisierung des Possessivsuffixes in andere Verwendungen aus. Sie geht jedoch auch nicht von einer Zweiteilung in possessive und nicht-possessive Verwendung aus. Vielmehr werden Konstruktionen mit Possessivsuffixen meines Erachtens aufgrund der Referentialität des Possessivsuffixes eingesetzt, unabhängig von einem possessiven oder nicht-possessiven Kontext.

Diese Referentialität ist dem Possessivsuffix inhärent, eine Zweiteilung ist meines Erachtens nie erfolgt, da das Possessivsuffix nie die Bedeutung possessiv ausgedrückt hat. Eine Veränderung in der Referentialität des Possessivsuffixes hingegen ist meines Erachtens nicht von der Hand zu weisen. Diese wird jedoch erst mit Hinzunahme der Textumgebung und der Bestandteile der einzelnen Konstruktionen, sprich der Realisierung des Referenzpunktes, deutlicher. Generell kann man bei den meisten Vorkommen von Konstruktionen mit Possessivsuffixen nicht von Form- und Funktionswandel, Obligatheit der Verwendung oder ausschließlicher Verwendung in der neuen Bedeutung bzw. von einer Grammatikalisierung sprechen. Je nach Verwendung jedoch werden mitunter einzelne Eigenschaften des Possessivsuffixes (anaphorische Verweiskraft, deiktische Verweiskraft, Mit-Kodierung von Topikalität als Pro-Form) gegenüber anderen hervorgehoben. Lediglich bei der Habeo-Konstruktion des Ungarischen oder den als 
finite Verbalformen eingesetzten Konstruktionen ist eine Grammatikalisierung nicht ganz von der Hand zu weisen. Diese betrifft jedoch die gesamte Konstruktion in ihrer neuen Form und Funktion, nicht jedoch das Possessivsuffix als einzelnen Bestandteil.

Wie in Kapitel 3 und 5.2 erwähnt entspricht das Anchoring der Funktion der Referenzpunkt-Konstruktion, wie sie in der Kognitiven Linguistik beschrieben wird, die Possessivkonstruktion im Allgemeinen wird dort als eine Art der ReferenzpunktKonstruktion angesehen. Somit erfolgt die Verankerung des Targets am Referenzpunkt in allen Arten von Possessivkonstruktionen: In unmarkierten Juxtapositionen (SuBS Subs), Genitiv-Konstruktionen mit dependent-marking (Subs-Gen Subs) und headmarking Konstruktionen mit Possessivsuffixen (SuBs-Px). Letzterem, dem Konstruktionstyp der ugrischen Sprachen, ist durch die Variation in der Realisierung des Referenzpunktes die Möglichkeit zur Weiterentwicklung gegeben. Diese Variation wird erst durch das Possessivsuffix der dritten Person ermöglicht, welche die mit Abstand am häufigsten kodierte Person ist, das am häufigsten verwendete Possessivsuffix dabei das der dritten Person Singular Einbesitz. In dessen Vorkommen befinden sich auch die meisten Variationen im Hinblick auf die in Kapitel 5.2 vorgestellten Mechanismen, und hier ist auch die Variation in der Hervorhebung der einzelnen Eigenschaften am häufigsten. Hier können drei Haupt-Tendenzen festgestellt werden:

- die Mit-Kodierung der Topikalität als Pro-Form wird hervorgehoben, um die Salienz des Referenzpunktes auf das Target zu übertragen;

- die gesamte persönliche referentielle Verweiskraft, das heißt die Kongruenz mit der Person, verschiebt sich zugunsten einer reinen Markierung einer grammatischen Relation, sodass ein semantisch leerer Marker verbleibt, dessen angezeigte grammatische Verbindung je nach Konstruktionsart mit der jeweiligen Lesart interpretiert wird, ähnlich dem Genitiv-Kasus;

- die Kodierung der Person verschiebt sich zugunsten der deiktischen Verweiskraft, sodass ein Marker verbleibt, welcher den jetzt in der Aufmerksamkeit des Sprechers befindlichen Referenten markiert, ähnlich einer Fokussierungs-Partikel;

Es sind somit drei Funktionen der Possessivsuffixe festzuhalten: rückweisend-anaphorisch, hinweisend-deiktisch und grammatisches Agreement. Beim Possessivsuffix der dritten Person führt dies möglicherweise bis hin zu einem Bruch in der Referentialität zwischen der Funktion als Personalmarker (zum Beispiel Anchoring - anaphorische Referenz), als grammatischer Marker (zum Beispiel Izafet - leere Referenz) sowie als Fokuspartikel (zum Beispiel Turn-Taking - deiktische Referenz).

In sogenannten semantic maps lassen sich solche Relationen zwischen verschiedenen Funktionen sowie Gemeinsamkeiten (aber auch Unterschiede) visualisieren, sodass etwaige Beziehungen und voneinander unabhängige Bereiche sowie mögliche Entwicklungspfade erkannt werden können. Semantic maps haben sich in den letzten Jahren als geeignete empirische Methode für Sprachvergleiche erwiesen, gleichzeitig weisen sie dabei Überschneidungsgebiete mit Grammatikalisierungstheorien auf (de Haan 2004: 1). Im Falle der vorliegenden Arbeit handelt es sich dabei jedoch eher um functional maps, da die Funktionen des Possessivsuffixes und nicht die Semantik der Possessivkonstruktion veranschaulicht werden sollen. 


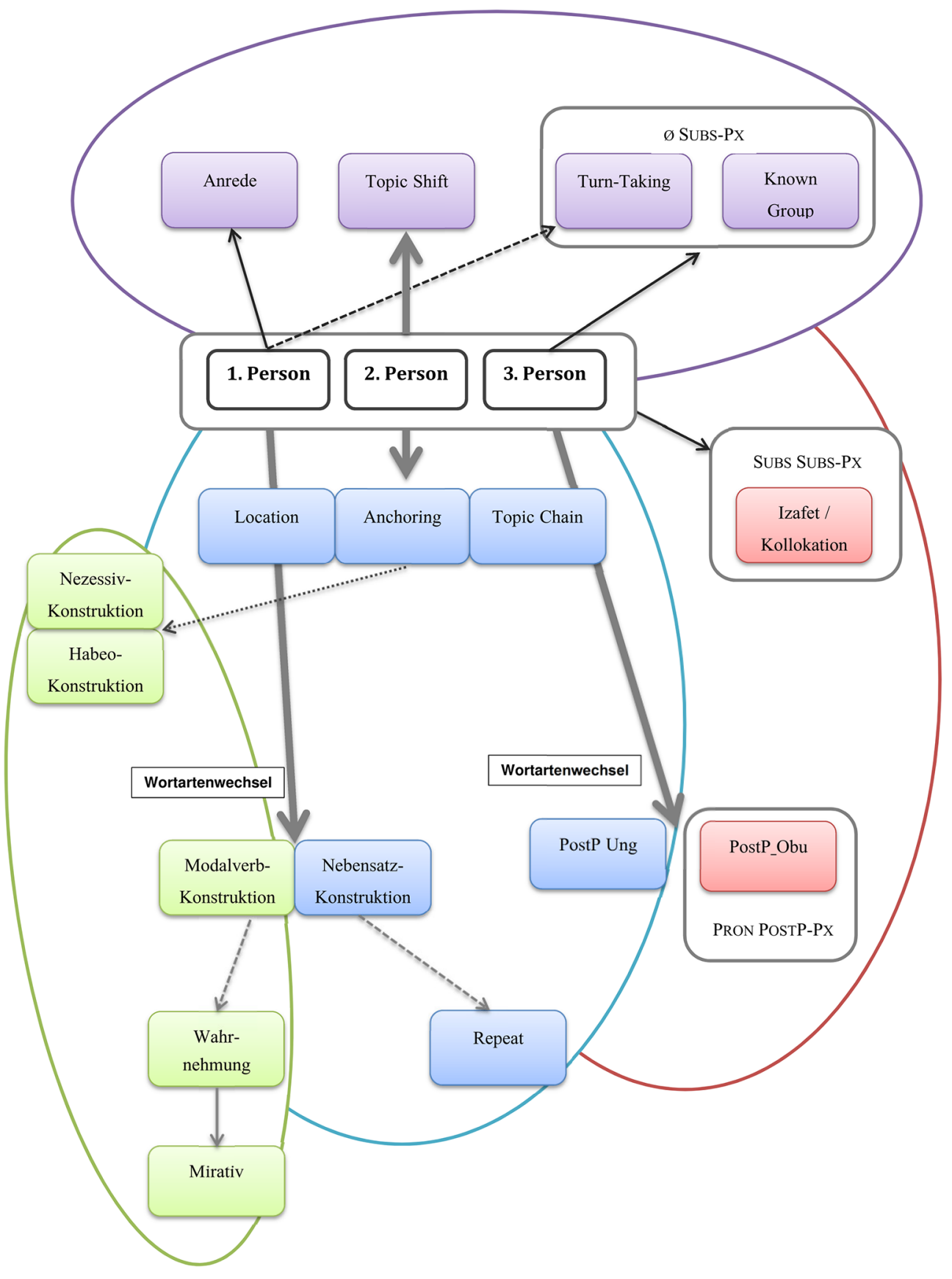

Abbildung 10: Semantic Map der Funktionen der Possessivsuffixe. (C) 2015 Gwen Eva Janda. Legende: Mechanismus im abgerundeten Rechteck; lila: deiktische Funktionen, blau: anaphorische Funktionen, rot: grammatisches Agreement, grün: in der Konstruktion grammatikalisiert. 
Die vorliegende Arbeit soll als exemplarisch für eine alternative Beschreibung von Konstruktionen mit Possessivsuffixen gelten und Anregungen für weitere neue Analyseansätze geben. Für die vorliegende Arbeit wurden vier ugrische Dialekte bzw. Sprachen ausgewählt. Die Berücksichtigung weiterer ob-ugrischer Dialekte oder auch ein Vergleich mit den Konstruktionen mit Possessivsuffixen anderer Sprachzweige der uralischen Sprachfamilie könnten weitere wertvolle Hinweise auf die Funktionen der Possessivsuffixe der uralischen Sprachen liefern. Geeignete Vergleichsmöglichkeiten könnten insbesondere die benachbarten permischen oder samojedischen Sprachzweig bieten (siehe dazu auch Kapitel 2.3.5). Die Ergebnisse einer vergleichenden Forschung innerhalb der uralischen Sprachfamilie könnten letztlich auch zu einer Umbenennung des Possessivsuffixes oder der Betrachtung mehrerer Kategorien statt eines einzelnen Paradigmas führen.

Auch die Textsorten sind nicht begrenzt auf die hier getroffene Auswahl - andere Genre können andere Verwendungsmuster aufweisen (verglichen Schulze 1997: 199), was sich als Tendenz auch im Material der vorliegenden Arbeiten gezeigt hat. Die Sachtexte des Ungarischen weisen fast ausschließlich Izafet-Konstruktionen auf, in den ungarischen Erzählungen hingegen erscheint viel mehr Variation im Hinblick auf die in Kapitel 5.2 vorgestellten Mechanismen. Letztlich ist auch die Datenanalyse nur exemplarisch, ein automatisiertes Tagging von syntaktischen, semantischen und pragmatischen Rollen könnte in kürzerer Zeit weitaus mehr Grundlagen für eine Analyse bereitstellen, und auch die zu analysierende Datenmenge könnte dadurch vervielfacht werden.

Die Ergebnisse der vorliegenden Arbeit zeigen, dass die Analyse der Possessivsuffixe außerhalb von Possessivität und Definitheit durchaus lohnend ist. 


\section{Abkürzungen der Sprachen, Dialekte und Subdialekte}

$\begin{array}{ll}\text { NM } & \text { Nord-Mansisch } \\ \text { KK } & \text { Kazym-Chantisch } \\ \text { SK } & \text { Surgut-Chantisch } \\ \text { VJ } & \text { Vasjugan-Chantisch } \\ \text { TJ } & \text { Tremjugan-Chantisch } \\ \text { HU } & \text { Ungarisch }\end{array}$

\section{Verzeichnis der verwendeten Glossen}

$\begin{array}{ll}\text { 1DU } & \text { 1. person dual } \\ \text { 1PL } & \text { 1. person plural } \\ \text { 1SG } & \text { 1. person singular } \\ \text { 2DU } & \text { 2. person dual } \\ \text { 2PL } & \text { 2. person plural } \\ \text { 2SG } & \text { 2. person singular } \\ \text { 3DU } & \text { 3. person dual } \\ \text { 3PL } & \text { 3. person plural } \\ \text { 3SG } & \text { 3. person singular } \\ \text { ABE } & \text { Abessive } \\ \text { ABL } & \text { Ablative } \\ \text { ACC } & \text { Accusative } \\ \text { ADJZR } & \text { Adjectivizer } \\ \text { ADVZR } & \text { Adverbizer } \\ \text { APP } & \text { Approximative } \\ \text { CAUS } & \text { Causative } \\ \text { COLL } & \text { Collective } \\ \text { COM } & \text { Comitative(-Instrumental) } \\ \text { COMPL } & \text { Completive } \\ \text { COMPR } & \text { Comparative } \\ \text { COND } & \text { Conditional } \\ \text { COP } & \text { Copula } \\ \text { CVB } & \text { Converb } \\ \text { DAT } & \text { Dative } \\ \text { DIM } & \text { Diminutive } \\ \text { DLAT } & \text { Dative-Lative } \\ \text { DU } & \text { Dual } \\ \text { DUR } & \text { Durative } \\ \text { EMPH } & \text { Emphasis } \\ \text { EVID } & \text { Evidential } \\ & \end{array}$




$\begin{array}{ll}\text { EXPL } & \text { Expletive } \\ \text { FREQ } & \text { Frequentative } \\ \text { HORT } & \text { Hortative } \\ \text { IMP } & \text { Imperative } \\ \text { INCH } & \text { Inchoative } \\ \text { INF } & \text { Infinitive } \\ \text { INSC } & \text { Instructive-Final } \\ \text { INST } & \text { Instrumental } \\ \text { INTR } & \text { Intransitive } \\ \text { INTS } & \text { Intensive } \\ \text { ITER } & \text { Iterative } \\ \text { LOC } & \text { Locative } \\ \text { MEL } & \text { Meliorative } \\ \text { MIR } & \text { Mirative } \\ \text { MOM } & \text { Momentary } \\ \text { NACT } & \text { Nomen Actionis (Action Nominal) } \\ \text { NEG } & \text { Negation, Negative } \\ \text { NOM } & \text { Nominative } \\ \text { NZER } & \text { Nominalizer } \\ \text { OBL } & \text { Oblique } \\ \text { OPT } & \text { Optative } \\ \text { ORD } & \text { Ordinal } \\ \text { PASS } & \text { Passive } \\ \text { POSBL } & \text { Possibility } \\ \text { PEJ } & \text { Pejorative } \\ \text { PFV } & \text { Perfective } \\ \text { PL } & \text { Plural } \\ \text { PREC } & \text { Precative } \\ \text { PROH } & \text { Prohibitive } \\ \text { PROPR } & \text { Proprietive } \\ \text { PRS } & \text { Present } \\ \text { PST } & \text { Past } \\ \text { PTCP } & \text { Participle } \\ \text { Q } & \text { Question Particle/Marker } \\ \text { QUOT } & \text { Quotative } \\ \text { RECP } & \text { Reciprocal } \\ \text { REFL } & \text { Reflexive } \\ \text { RES } & \text { Resultative } \\ \text { SG } & \text { Singular } \\ \text { SUBL } & \text { Sublativ } \\ \text { SUP } & \text { Superlative } \\ \text { TR } & \text { Transitive } \\ \text { TRNS } & \text { Translative } \\ \text { VOC } & \text { Vocative } \\ \text { VZER } & \text { Verbalizer } \\ & \end{array}$




\section{Abbildungsverzeichnis}

Abbildung 1: $\quad$ Siedlungsgebiete der Mansen, eigene Adaption nach Csepregi 1998: 48.

Abbildung 2: $\quad$ Siedlungsgebiete der Chanten, eigene Adaption nach Csepregi 1998: 60.

Abbildung 3: Verteilung der ungarischen Dialekte, eigene Adaption nach Hajdú, Péter/ Domokos, Péter (Hg.) 1987.

Abbildung 4: Rekonstruierte Formen der Personalpronomen und Personalsuffixe für das Ur-Ob-Ugrische, eigene Adaption in IPA nach Honti 1998: 234.

Abbildung 5: $\quad$ Das Referenzpunkt-Modell nach Langacker 1993: 6.

Abbildung 6: Skala Salienz (»accessibility«) des Topik und Anaphorisches Verweismittel nach Givón 1983: 17f.

Abbildung 7: Das Referenzpunkt-Modell nach Langacker 1993 abgeleitet auf den Text. Eigene Adaption.

Abbildung 8: Schriftprobe aus den Aufzeichnungen von Valerij Nikolajevič Černecov. ( 2012 OUL, http://www.oudb.gwi.uni-muenchen.de/index.php?abfrage=fieldwork_ chernetsov (Zugriff vom 22.05.2017).

Abbildung 9: Überschneidung der Wortart Substantiv und Postposition in Munkácsi/ Kálmán 1986. @ 2012 OUL, http://www.oudb.gwi.uni-muenchen.de/index.php ?abfrage=munka\&subnavi=edictionary (Zugriff vom 22.05.2017).

Abbildung 10: Semantic Map der Funktionen der Possessivsuffixe. ( 2015 Gwen Eva Janda. Legende: Mechanismus im abgerundeten Rechteck; lila: deiktische Funktionen, blau: anaphorische Funktionen, rot: grammatisches Agreement, grün: in der Konstruktion grammatikalisiert. 


\section{Tabellenverzeichnis}

Tabelle 1: Dialektale Gliederung des Mansischen.

Tabelle 2: $\quad$ Dialektale Gliederung des Chantischen.

Tabelle 3: $\quad$ Schematische Darstellung der in der Possessivkonstruktion kodierten

Referenten.

Tabelle 4: $\quad$ Paradigma der Possessivsuffixe des Nord-Mansischen.

Tabelle 5: $\quad$ Paradigma der Possessivsuffixe des Nord-Chantischen (am Beispiel des

Kazym-Dialektes).

Tabelle 6: $\quad$ Paradigma der Possessivsuffixe des Ost-Chantischen (am Beispiel des Surgut-Dialektes).

Tabelle 7: $\quad$ Paradigma der Possessivsuffixe des Ungarischen.

Tabelle 8: $\quad$ Segmentierung der Form -anəl dritte Person Plural Einbesitz und -ananəl dritte Person Plural Mehrbesitz.

Tabelle 9: $\quad$ Belege für Habeo-Konstruktionen des Ungarischen aus dem Korpus.

Tabelle 10: Typen und Vorkommen der attributiven Possessivkonstruktion in den uralischen Sprachen (Tabelle, Beispiele und Notation nach Honti 2007b: 55f.).

Tabelle 11: Konstruktionstypen der attributiven Possessivkonstruktion in der vorliegenden Arbeit.

Tabelle 12: Konstruktionen mit Possessivsuffix in der vorliegenden Arbeit.

Tabelle 13: Korrelation von syntaktischer, semantischer und pragmatischer Rolle nach Givón 1983: 22f.

Tabelle 14: Verteilung der Korpusbelege auf die einzelnen Konstruktionen mit Possessivsuffix.

Tabelle 15: $\quad$ Realisierungen des Modifizierers in der Konstruktion Subs-Px im Korpus der vorliegenden Arbeit.

Tabelle 16: Konstruktionen des Ungarischen mit obligatorischer Verwendung des Possessivsuffixes.

Tabelle 17: $\quad$ Schematische Darstellung der Bestandteile der Possessivkonstruktion.

Tabelle 18: In der Possessivkonstruktion des Ungarischen ausgedrückte Relationen nach Kenesei/Vago/Fenyvesi (Hg.) 1998: 160.

Tabelle 19: Im Korpus belegte Verwandtschaftstermini.

Tabelle 20: Realisierungen des Modifizierers in der Konstruktion VN-Px im Korpus der vorliegenden Arbeit.

Tabelle 21: $\quad$ Struktur der Modalverb-Konstruktionen des Ungarischen.

Tabelle 22: $\quad$ Struktur der Konstruktion PersPr Subs-Px mit Postposition gegenüber der Konstruktion PersPr Post-Px.

Tabelle 23: $\quad$ Realisierungen des Modifizierers in der Konstruktion PostP-Px im Korpus der vorliegenden Arbeit.

Tabelle 24: Zusammenführung von Konstruktionen und Mechanismen.

Tabelle 25: $\quad$ Paradigma der Possessivsuffixe des Nord-Mansischen.

Tabelle 26: Paradigma der Possessivsuffixe des Nord-Chantischen (am Beispiel des Kazym-Dialektes).

Tabelle 27: $\quad$ Paradigma der Possessivsuffixe des Ost-Chantischen (am Beispiel des Surgut-Dialektes).

Tabelle 28: $\quad$ Paradigma der Possessivsuffixe des Ungarischen.

Tabelle 29: $\quad$ Verteilung der Mechanismen auf die einzelnen Sprachen bzw. Dialekte.

Tabelle 30: Auswirkung der Realisierung des Referenzpunktes nach Konstruktion. 


\section{Quellen}

\section{Primärliteratur (Korpus)}

HU_text_001 = A vasfejü ember. Eisenkopf, Quelle: Török, Károly, in: Arany/Gyulai (Hg.) 1872, 375-388, URL: http://www.gutenberg.org/files/43324/43324-h/43324-h.htm, Quelle Annotation: Eigene Annotation, Übersetzung Deutsch/Englisch, URL: http://www.sagen.at/ texte/maerchen/maerchen_ungarn/ungarn_volksmaerchen/eisenkopf.html (Zugriff vom 17.04.2017).

HU_text_002 = A halál és a vén asszony. Der Tod und die Alte, Quelle: Török, Károly, in: Arany/ Gyulai (Hg.) 1872, 436-439, URL: http://www.gutenberg.org/files/43324/43324-h/43324-h. htm, Quelle Annotation: Eigene Annotation, Übersetzung Deutsch/Englisch, URL: http:// www.sagen.at/texte/maerchen/maerchen_ungarn/ungarn_volksmaerchen/tod_alte.html (Zugriff vom 10.05.2017).

HU_text_003 = Újra rekordévet zár az Erasmus. Weiteres Rekordjahr für Erasmus, Quelle: Europäische Kommision Press Release Database (10.07.2014), URL: http://europa.eu/rapid/press-release_IP-14-821_hu.htm, Quelle Annotation: Eigene Annotation, Übersetzung Deutsch/Englisch, URL: http://europa.eu/rapid/press-release_IP-14-821_de.htm (Zugriff vom 23.07.2014).

HU_text_004 = Az új felsőoktatási reformstratégia lényege: korszerüsités és foglalkoztathatóság. Reform der Hochschulbildung: Modernisierung und Beschäftigungsfähigkeit sind Kernstück der neuen Strategie, Quelle: Europäische Kommision Press Release Database (20.09.2011), URL: http://europa.eu/rapid/press-release_IP-11-1043_hu.htm, Quelle Annotation: Eigene Annotation, Übersetzung Deutsch/Englisch, URL: http://europa.eu/rapid/press-release_IP-111043_de.htm (Zugriff vom 24.07.2014).

HU_text_005 $=A z$ aranyhajú tündér királykisasszony. Feenprinzessin Goldhaar. Quelle: Török, Károly, in: Arany/Gyulai (Hg.) 1872, 440-455, URL: http://www.gutenberg.org/ files/43324/43324-h/43324-h.htm, Quelle Annotation: Eigene Annotation, Übersetzung Deutsch/Englisch, URL: http://www.sagen.at/texte/maerchen/maerchen_ungarn/ungarn_ volksmaerchen/goldhaar.html (Zugriff vom 30.07.2014).

HU_text_006 = 60 egyetem kapja meg az »európai mesterszintü forditóképzés « minőségjelölést. Europäischer Master Übersetzen: 60 Hochschulen erhalten EMT-Gütezeichen, Quelle: Europäische Kommision Press Release Database (02.06.2014), URL: http://europa.eu/rapid/press-release_ IP-12-1233_hu.htm, Quelle Annotation: Eigene Annotation, Übersetzung Deutsch/Englisch, URL: http://europa.eu/rapid/press-release_IP-14-625_de.htm (Zugriff vom 15.08.2014).

HU_text_007 = János királyfi és Szélike. Prinz Johann und Prinzessin Windhauch, Quelle: Török, Károly, in: Arany/Gyulai (Hg.) 1872, 389-397, Quelle Annotation: Eigene Annotation, Übersetzung Deutsch/Englisch, URL: http://www.sagen.at/texte/maerchen/maerchen_ungarn/ ungarn_volksmaerchen/prinz.html (Zugriff vom 13.08.2014).

HU_text_008 = A víztorony. Der Wasserturm, Quelle: Tar, Sándor, in: Kunze 1999, 138-151, Quelle Annotation: Eigene Annotation, Übersetzung Deutsch/Englisch: Kunze 1999, 138-151.

HU_text_009 = Az aranyhajú kertészbojtár. Der goldhaarige Gärtnersbursche, Quelle: Merényi 1861, 111-122, Quelle Annotation: Eigene Annotation, Übersetzung Deutsch/Englisch, URL: http://www.sagen.at/texte/maerchen/maerchen_ungarn/ungarn_volksmaerchen/gaertnersbursche.html (Zugriff vom 30.08.2014).

HU_text_010 = Azárnyék. Der Schatten, Quelle: Mészöly, Miklós, in: Kunze 1999, 126-137, Quelle Annotation: Eigene Annotation, Übersetzung Deutsch/Englisch: Kunze 1999, 126-137.

KK_satz_0001 $=j \theta \int . . . j \theta \int \partial \beta$ manat $\beta$ ont moxti. Der Weg... der Weg geht durch den Wald, Quelle: Schön, Zsófia, Postpositionale Datenbank, 2015, Quelle Annotation und Übersetzung Deutsch/ Englisch: Zsófia Schön/Eigene Annotation und Übersetzung. 
KK_satz_0002 = tu $\beta$ manəs xo:tat $\beta$ enti. Er ging bis zu seinem Haus, Quelle: Schön, Zsófia, Postpositionale Datenbank, 2015, Quelle Annotation und Übersetzung Deutsch/Englisch: Zsófia Schön/Eigene Annotation und Übersetzung.

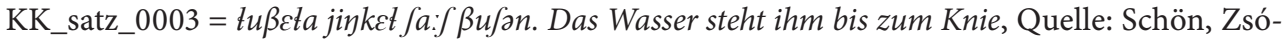
fia, Postpositionale Datenbank, 2015, Quelle Annotation und Übersetzung Deutsch/Englisch: Zsófia Schön/Eigene Annotation und Übersetzung.

KK_satz_0004 = luß mo:nisict mo:simat ju:pijan mun ja:sisuß $\int a: j$. Nachdem er das Märchen erzählt hatte, tranken wir Tee, Quelle: Schön, Zsófia, Postpositionale Datenbank, 2015, Quelle Annotation und Übersetzung Deutsch/Englisch: Zsófia Schön/Eigene Annotation und Übersetzung.

KK_satz_0005 = jefeß manat $\beta$ ent muxti. Unser Weg geht durch den Wald, Quelle: Schön, Zsófia, Postpositionale Datenbank, 2015, Quelle Annotation und Übersetzung Deutsch/Englisch: Zsófia Schön/Eigene Annotation und Übersetzung.

KK_satz_0006 $=$ jefem $\beta$ ent muxti mant. Mein Weg geht durch den Wald, Quelle: Schön, Zsófia, Postpositionale Datenbank, 2015, Quelle Annotation und Übersetzung Deutsch/Englisch: Zsófia Schön/Eigene Annotation und Übersetzung.

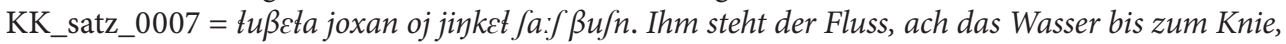
Quelle: Schön, Zsófia, Postpositionale Datenbank, 2015, Quelle Annotation und Übersetzung Deutsch/Englisch: Zsófia Schön/Eigene Annotation und Übersetzung.

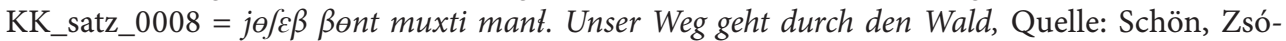
fia. Postpositionale Datenbank, 2015, Quelle Annotation und Übersetzung Deutsch/Englisch: Zsófia Schön/Eigene Annotation und Übersetzung.

KK_satz_0009 = xə:tt ßonta juxtəs. Er kam bis zu seinem Haus, Quelle: Schön, Zsófia, Postpositionale Datenbank, 2015, Quelle Annotation und Übersetzung Deutsch/Englisch: Zsófia Schön/ Eigene Annotation und Übersetzung.

KK_satz_0010 = mo:nisiat mo:simat ju:pijan mun fa:j ja:sisuß. Nachdem er sein Märchen erzählt hatte, tranken wir Tee, Quelle: Schön, Zsófia, Postpositionale Datenbank, 2015, Quelle Annotation und Übersetzung Deutsch/Englisch: Zsófia Schön/Eigene Annotation und Übersetzung.

KK_satz_0011 = tuß ma: xosjama jit. Er kommt zu mir, Quelle: Schön, Zsófia, Postpositionale Datenbank, 2015, Quelle Annotation und Übersetzung Deutsch/Englisch: Zsófia Schön/Eigene Annotation und Übersetzung.

KK_satz_0012 = ma: tunmeman jutan nemət-xojat ants:m. Als ich hineinging, war niemand $z u$ Hause, Quelle: Schön, Zsófia, Postpositionale Datenbank, 2015, Quelle Annotation und Übersetzung Deutsch/Englisch: Zsófia Schön/Eigene Annotation und Übersetzung.

KK_satz_0013 = lupmat ju:pijan kim ctas. Nachdem ergesprochen hatte, ging er raus, Quelle: Schön, Zsófia, Postpositionale Datenbank, 2015, Quelle Annotation und Übersetzung Deutsch/ Englisch: Zsófia Schön/Eigene Annotation und Übersetzung.

KK_satz_0014 = multitat lupmat ju:pijan tuß kim ctas. Nachdem er etwas gesagt hatte, ging er raus, Quelle: Schön, Zsófia, Postpositionale Datenbank, 2015, Quelle Annotation und Übersetzung Deutsch/Englisch: Zsófia Schön/Eigene Annotation und Übersetzung.

KK_satz_0015 = Bo:n'isiam-ot akatmeman jerta jis. Als ich Beeren sammelte, regnete es, Quelle: Schön, Zsófia, Postpositionale Datenbank, 2015, Quelle Annotation und Übersetzung Deutsch/ Englisch: Zsófia Schön/Eigene Annotation und Übersetzung.

KK_satz_0016 = ma: nay e: $\beta$ attana a:jəak. Ich bin jünger als du, Quelle: Schön, Zsófia, Postpositionale Datenbank, 2015, Quelle Annotation und Übersetzung Deutsch/Englisch: Zsófia Schön/ Eigene Annotation und Übersetzung.

KK_satz_0017 = tuß me/ti maratan ma: manti pifem ants:m. Solange er krank ist, kann ich nicht kommen, Quelle: Schön, Zsófia, Postpositionale Datenbank, 2015, Quelle Annotation und Übersetzung Deutsch/Englisch: Zsófia Schön/Eigene Annotation und Übersetzung.

KK_satz_0018 = ßo:njisiam-ut aktum maremon is'a jertas. Die ganze Zeit, während ich Beeren sammelte, regnete es, Quelle: Schön, Zsófia, Postpositionale Datenbank, 2015, Quelle Annotation und Übersetzung Deutsch/Englisch: Zsófia Schön/Eigene Annotation und Übersetzung. 
KK_satz_0019 = tep timofejan it xөyxas tuß xusjata xitneyat sii xөxat. Als Timofej ausstieg, rannte seine Enkelin zu ihm, Quelle: Schön, Zsófia, Postpositionale Datenbank, 2015, Quelle Annotation und Übersetzung Deutsch/Englisch: Zsófia Schön/Eigene Annotation und Übersetzung.

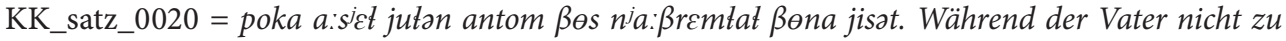
Hause war, wurden die Kinder groß, Quelle: Schön, Zsófia, Postpositionale Datenbank, 2015, Quelle Annotation und Übersetzung Deutsch/Englisch: Zsófia Schön/Eigene Annotation und Übersetzung.

KK_satz_0021 = a:ykem a:toy sa: repataja mans. Meine Mutter ging morgens zur Arbeit, Quelle: Schön, Zsófia, Postpositionale Datenbank, 2015, Quelle Annotation und Übersetzung Deutsch/ Englisch: Zsófia Schön/Eigene Annotation und Übersetzung.

KK_satz_0022 = Butitam Bont xußat ara mansat. Die Rentiere gingen durch den Wald, Quelle: Schön, Zsófia, Postpositionale Datenbank, 2015, Quelle Annotation und Übersetzung Deutsch/ Englisch: Zsófia Schön/Eigene Annotation und Übersetzung.

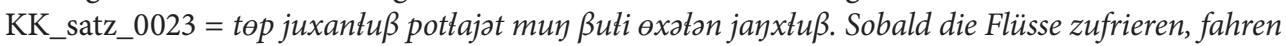
wir im Rentierschlitten, Quelle: Schön, Zsófia, Postpositionale Datenbank, 2015, Quelle Annotation und Übersetzung Deutsch/Englisch: Zsófia Schön/Eigene Annotation und Übersetzung.

KK_satz_0024 = 3ofijajan Bot tajisja sticpanovnajan xusja. Zsófi wohnt bei Taisja Stepanovna, Quelle: Schön, Zsófia, Postpositionale Datenbank, 2015, Quelle Annotation und Übersetzung Deutsch/Englisch: Zsófia Schön/Eigene Annotation und Übersetzung.

KK_satz_0025 = iket juxi juxttat ßonta imet jutan a:tat $\beta$ os. Bis ihr Ehemann nach Hause kommt, war seine Frau allein zu Hause, Quelle: Schön, Zsófia, Postpositionale Datenbank, 2015, Quelle Annotation und Übersetzung Deutsch/Englisch: Zsófia Schön/Eigene Annotation und Übersetzung.

KK_satz_0026 = Butcn juxan xonəya juxttatan jink ja:nitiz. Als das Rentier ans Flussufer kommt, beginnt es zu trinken, Quelle: Schön, Zsófia, Postpositionale Datenbank, 2015, Quelle Annotation und Übersetzung Deutsch/Englisch: Zsófia Schön/Eigene Annotation und Übersetzung.

KK_satz_0027 = tөp $\beta u t i t u \beta$ juxatsat a:j e:ßije si xoxatmas. Als die Rentiere kamen, rannte das kleine Mädchen zu ihnen, Quelle: Schön, Zsófia, Postpositionale Datenbank, 2015, Quelle Annotation und Übersetzung Deutsch/Englisch: Zsófia Schön/Eigene Annotation und Übersetzung.

KK_satz_0028 = xon jerta jit tonttan morti mußa mantat. Sobald der Regen kommt, fliegen die Gänse in den Süden, Quelle: Schön, Zsófia, Postpositionale Datenbank, 2015, Quelle Annotation und Übersetzung Deutsch/Englisch: Zsófia Schön/Eigene Annotation und Übersetzung.

KK_satz_0029 = tuß xə:nayałan tuß iket tojts. Neben ihr stand ihr Mann, Quelle: Schön, Zsófia, Postpositionale Datenbank, 2015, Quelle Annotation und Übersetzung Deutsch/Englisch: Zsófia Schön/Eigene Annotation und Übersetzung.

KK_satz_0030 = top $\beta$ utitu $\beta$ juxtsat a:j e: $\beta i$ tu $\beta$ xusjeta xoxatmas. Als die Rentiere kamen, rannte das kleine Mädchen zu ihnen, Quelle: Schön, Zsófia, Postpositionale Datenbank, 2015, Quelle Annotation und Übersetzung Deutsch/Englisch: Zsófia Schön/Eigene Annotation und Übersetzung.

KK_satz_0031 = tep tiimofejan juxtas tuß je:fetteta xitneyat sji xөxatmas. Als Timofej kam, rannte seine Enkelin zu ihm, Quelle: Schön, Zsófia, Postpositionale Datenbank, 2015, Quelle Annotation und Übersetzung Deutsch/Englisch: Zsófia Schön/Eigene Annotation und Übersetzung.

KK_satz_0032 = a:ykem a:toy repataja mans. Meine Mutter ging morgens zur Arbeit, Quelle: Schön, Zsófia, Postpositionale Datenbank, 2015, Quelle Annotation und Übersetzung Deutsch/ Englisch: Zsófia Schön/Eigene Annotation und Übersetzung.

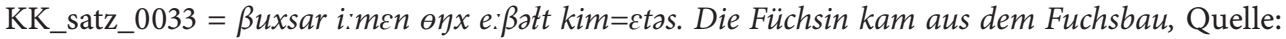
Schön, Zsófia, Postpositionale Datenbank, 2015, Quelle Annotation und Übersetzung Deutsch/ Englisch: Zsófia Schön/Eigene Annotation und Übersetzung.

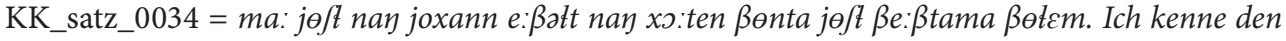
Weg zwischen deinem Haus und dem Fluss nicht so gut, Quelle: Schön, Zsófia, Postpositionale Datenbank, 2015, Quelle Annotation und Übersetzung Deutsch/Englisch: Zsófia Schön/Eigene Annotation und Übersetzung. 
KK_satz_0035 = a:sie $\beta$ jutan an $\beta$ otum mar. Während der Vater nicht $z u$ Hause war, wurden die Kinder groß... sehr groß, Quelle: Schön, Zsófia, Postpositionale Datenbank, 2015, Quelle Annotation und Übersetzung Deutsch/Englisch: Zsófia Schön/Eigene Annotation und Übersetzung.

KK_satz_0036 = a:yka:ykem tuß pitraton $\beta$ ot. Die Großmutter wohnt neben ihr, Quelle: Schön, Zsófia, Postpositionale Datenbank, 2015, Quelle Annotation und Übersetzung Deutsch/Englisch: Zsófia Schön/Eigene Annotation und Übersetzung.

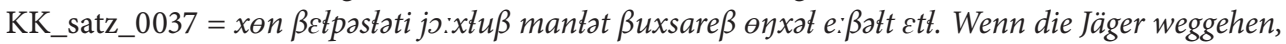
kommt der Fuchs aus seinem Bau heraus, Quelle: Schön, Zsófia, Postpositionale Datenbank, 2015, Quelle Annotation und Übersetzung Deutsch/Englisch: Zsófia Schön/Eigene Annotation und Übersetzung.

KK_satz_0038 = imen xo:nəyən tuß iket to:tis. Neben der Ehefrau stand ihr Mann, Quelle: Schön, Zsófia, Postpositionale Datenbank, 2015, Quelle Annotation und Übersetzung Deutsch/Englisch: Zsófia Schön/Eigene Annotation und Übersetzung.

KK_satz_0039 = tam xo:ton tum xo:tt e: $\beta \partial t$ ßonfok. Dieses Haus ist viel größer als jenes Haus, Quelle: Schön, Zsófia, Postpositionale Datenbank, 2015, Quelle Annotation und Übersetzung Deutsch/Englisch: Zsófia Schön/Eigene Annotation und Übersetzung.

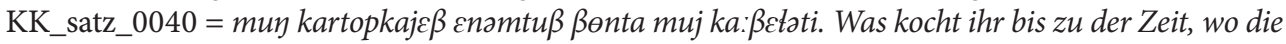
Kartoffeln gewachsen sind? Quelle: Schön, Zsófia, Postpositionale Datenbank, 2015, Quelle Annotation und Übersetzung Deutsch/Englisch: Zsófia Schön/Eigene Annotation und Übersetzung. KK_satz_0041 = imen iket juxtti Bonta tetot te:fatt. Die Ehefrau kocht Essen für die Ankunft des Ehemannes, Quelle: Schön, Zsófia, Postpositionale Datenbank, 2015, Quelle Annotation und Übersetzung Deutsch/Englisch: Zsófia Schön/Eigene Annotation und Übersetzung.

KK_satz_0042 = zofi taijsja stepanovnajon xusja ßot. Zsófi wohnt bei Taisja Stepanovna, Quelle: Schön, Zsófia, Postpositionale Datenbank, 2015, Quelle Annotation und Übersetzung Deutsch/ Englisch: Zsófia Schön/Eigene Annotation und Übersetzung.

KK_satz_0043 = tam xo:tən jama ßonfak tumat e:ßatt. Dieses Haus ist viel größer als jenes, Quelle: Schön, Zsófia, Postpositionale Datenbank, 2015, Quelle Annotation und Übersetzung Deutsch/ Englisch: Zsófia Schön/Eigene Annotation und Übersetzung.

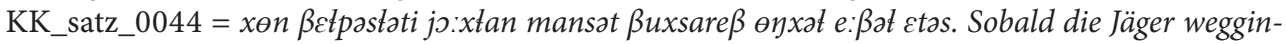
gen, kam die Füchsin aus dem Bau heraus, Quelle: Schön, Zsófia, Postpositionale Datenbank, 2015, Quelle Annotation und Übersetzung Deutsch/Englisch: Zsófia Schön/Eigene Annotation und Übersetzung.

KK_satz_0045 = juxanton po:tti jupijon jeøk xußat jayxłət oxətən. Nachdem die Flüsse zugefroren sind können sie mit dem Schlitten auf dem Eis entlangfahren, Quelle: Schön, Zsófia, Postpositionale Datenbank, 2015, Quelle Annotation und Übersetzung Deutsch/Englisch: Zsófia Schön/ Eigene Annotation und Übersetzung.

KK_satz_0046 = tam xっ:tan ßonfak tum xっ:tan e:ßat. Dieses Haus ist viel größer als jenes, Quelle: Schön, Zsófia, Postpositionale Datenbank, 2015, Quelle Annotation und Übersetzung Deutsch/ Englisch: Zsófia Schön/Eigene Annotation und Übersetzung.

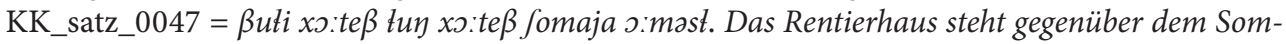
merhaus, Quelle: Schön, Zsófia, Postpositionale Datenbank, 2015, Quelle Annotation und Übersetzung Deutsch/Englisch: Zsófia Schön/Eigene Annotation und Übersetzung.

KK_satz_0048=ma: jefen a:təma ßotem xo:ten pa: juxanen kutən. Ich kenne den Weg vom Fluss zu deinem Haus schlecht, Quelle: Schön, Zsófia, Postpositionale Datenbank, 2015, Quelle Annotation und Übersetzung Deutsch/Englisch: Zsófia Schön/Eigene Annotation und Übersetzung.

KK_satz_0049 = a:jta:yken oß sa:ja xan'eməs. Die Maus versteckte sich hinter der Tür, Quelle: Schön, Zsófia, Postpositionale Datenbank, 2015, Quelle Annotation und Übersetzung Deutsch/ Englisch: Zsófia Schön/Eigene Annotation und Übersetzung.

KK_satz_0050 = ma: pot/ta nencm je:sialt xoxatsum. Ich lief der Postbotin entgegen, Quelle: Schön, Zsófia, Postpositionale Datenbank, 2015, Quelle Annotation und Übersetzung Deutsch/Englisch: Zsófia Schön/Eigene Annotation und Übersetzung. 
KK_satz_0051 = sa:kkereß xutas mjod ta:yxatan ki sa:kkar jukana puna. Unser Zucker ist aus, Wenn Du Honig möchtest, nimm es an Stelle von Zucker! Quelle: Schön, Zsófia, Postpositionale Datenbank, 2015, Quelle Annotation und Übersetzung Deutsch/Englisch: Zsófia Schön/Eigene Annotation und Übersetzung.

KK_satz_0052 = tiß juxtijitti pitmem jupijon ma: a:rsir xantet ufa ßersum. Nachdem ich anfing hierher zu kommen, habe ich viele Chanten kennengelernt, Quelle: Schön, Zsófia, Postpositionale Datenbank, 2015, Quelle Annotation und Übersetzung Deutsch/Englisch: Zsófia Schön/ Eigene Annotation und Übersetzung.

KK_satz_0053 = ti $\beta$ juxətmet jupijon mun jaxa $\beta$ o:nisism-ut $\beta$ o:sisu $\beta$. Nachdem sie angekommen waren, sammelten wir gemeinsam Beeren, Quelle: Schön, Zsófia, Postpositionale Datenbank, 2015, Quelle Annotation und Übersetzung Deutsch/Englisch: Zsófia Schön/Eigene Annotation und Übersetzung.

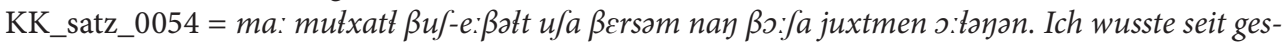
tern, dass Du in die Stadt gekommen warst, Quelle: Schön, Zsófia, Postpositionale Datenbank, 2015, Quelle Annotation und Übersetzung Deutsch/Englisch: Zsófia Schön/Eigene Annotation und Übersetzung.

KK_satz_0055 = a:mp na:ßermas xs:t e:ßatt $\beta u t i t a t x u s i$, Der Hund lief vom Haus zu den Rentieren, Quelle: Schön, Zsófia, Postpositionale Datenbank, 2015, Quelle Annotation und Übersetzung Deutsch/Englisch: Zsófia Schön/Eigene Annotation und Übersetzung.

KK_satz_0056 = juxanen po:tti jetpijan muך xusjaßa juxtajitłat. Bevor der Fluss zufriert, kommt man zu uns, Quelle: Schön, Zsófia, Postpositionale Datenbank, 2015, Quelle Annotation und Übersetzung Deutsch/Englisch: Zsófia Schön/Eigene Annotation und Übersetzung.

KK_satz_0057 = a:mpem ßutitat pe:ta xөxətməs. Mein Hund lief zu den Rentieren, Quelle: Schön, Zsófia, Postpositionale Datenbank, 2015, Quelle Annotation und Übersetzung Deutsch/Englisch: Zsófia Schön/Eigene Annotation und Übersetzung.

KK_satz_0058 = mantat jetpijan le:najen ma: xosjema joxtijits pa: jam ßotum ßerti. Vor ihrer Abreise kam Lena zu mir, um sich zu verabschieden, Quelle: Schön, Zsófia, Postpositionale Datenbank, 2015, Quelle Annotation und Übersetzung Deutsch/Englisch: Zsófia Schön/Eigene Annotation und Übersetzung.

KK_satz_0059 = ma: mulxatt $\beta u f-e: \beta a t t ~ u f a \beta \varepsilon r s a m$ xoti nay $\beta a: \int a$ juxtmen. Ich wusste es seit gestern, dass du in die Stadt gekommen warst, Quelle: Schön, Zsófia, Postpositionale Datenbank, 2015, Quelle Annotation und Übersetzung Deutsch/Englisch: Zsófia Schön/Eigene Annotation und Übersetzung.

KK_satz_0060 = ma: tałay xatta jutan xasisum soxtan pesti pati. Ich blieb den ganzen Tag zu Hause, um die Kleider zu waschen, Quelle: Schön, Zsófia, Postpositionale Datenbank, 2015, Quelle Annotation und Übersetzung Deutsch/Englisch: Zsófia Schön/Eigene Annotation und Übersetzung.

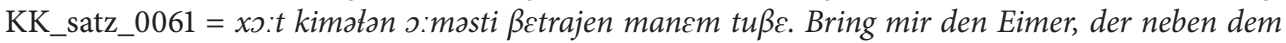
Haus steht, Quelle: Schön, Zsófia, Postpositionale Datenbank, 2015, Quelle Annotation und Übersetzung Deutsch/Englisch: Zsófia Schön/Eigene Annotation und Übersetzung.

KK_satz_0062 = tuße ma: xosjama ßetrajan... Bring mir den Eimer..., Quelle: Schön, Zsófia, Postpositionale Datenbank, 2015, Quelle Annotation und Übersetzung Deutsch/Englisch: Zsófia Schön/Eigene Annotation und Übersetzung.

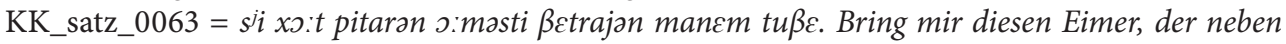
dem Haus steht, Quelle: Schön, Zsófia, Postpositionale Datenbank, 2015, Quelle Annotation und Übersetzung Deutsch/Englisch: Zsófia Schön/Eigene Annotation und Übersetzung.

KK_satz_0064 = tuß $\beta$ ot ma: xo:t punteman. Er steht neben meinem Haus, Quelle: Schön, Zsófia, Postpositionale Datenbank, 2015, Quelle Annotation und Übersetzung Deutsch/Englisch: Zsófia Schön/Eigene Annotation und Übersetzung.

KK_satz_0065 = Bertxo xo:t puntetan multi $\beta \varepsilon r a t$. Der Onkel macht etwas neben seinem Haus, Quelle: Schön, Zsófia, Postpositionale Datenbank, 2015, Quelle Annotation und Übersetzung Deutsch/Englisch: Zsófia Schön/Eigene Annotation und Übersetzung. 
KK_satz_0066 = Bertem xs:tt puycton motti $\beta \varepsilon r t$. Mein Onkel macht etwas neben seinem Haus, Quelle: Schön, Zsófia, Postpositionale Datenbank, 2015, Quelle Annotation und Übersetzung Deutsch/Englisch: Zsófia Schön/Eigene Annotation und Übersetzung.

KK_text_001 = nicti-ka:ti imi. Die suchende Frau, Quelle: Молданов 2001, 127, Quelle Annotation und Übersetzung Deutsch/Englisch: OUDB Kazym Khanty Corpus, Text ID 1000, bearbeitet von Schön, Zsófia, URL: http://www.oudb.gwi.uni-muenchen.de/?cit=1000 (Zugriff vom 18.04.2017).

KK_text_002 = jisən ka:saman ßuti antum ßos. In der alten Zeit gab es am Kazym kein Rentier, Quelle: Соловар 1995, 75, Quelle Annotation und Übersetzung Deutsch/Englisch: OUDB Kazym Khanty Corpus, Text ID 1027, bearbeitet von Schön, Zsófia, URL: http://www.oudb. gwi.uni-muenchen.de/?cit=1027 (Zugriff vom 18.04.2017).

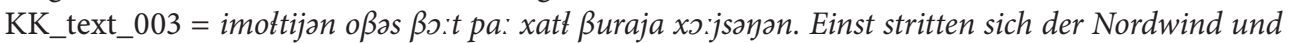
die Sonne, welcher der stärkste sei, Quelle: IPA (Hg.) 1999 »The north wind and the sun«, in: International Phonetic Association (Hg.) 1999, 39, Quelle Annotation und Übersetzung Deutsch/Englisch: OUDB Kazym Khanty Corpus, Text ID 1132, bearbeitet von Schön, Zsófia, URL: http://www.oudb.gwi.uni-muenchen.de/?cit=1132 (Zugriff vom 18.04.2017).

KK_text_004 = a:j kurininia. Kleine kurininia, Quelle: Rédei 1968, 94-95, Quelle Annotation und Übersetzung Deutsch/Englisch: OUDB Kazym Khanty Corpus, Text ID 1030, bearbeitet von Schön, Zsófia, URL: http://www.oudb.gwi.uni-muenchen.de/?cit=1030 (Zugriff vom 18.04.2017).

KK_text_005 = a:j mっ:n'jis-ne. Die kleine Mo:njsi-Frau, Quelle: Себурова (Hg.) 2009, 49-53, Quelle Annotation und Übersetzung Deutsch/Englisch: OUDB Kazym Khanty Corpus, Text ID 1119, bearbeitet von Schön, Zsófia, URL: http://www.oudb.gwi.uni-muenchen.de/?cit=1119 (Zugriff vom 18.04.2017).

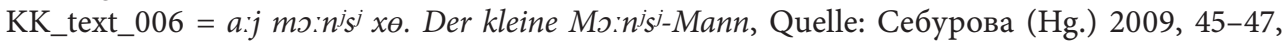
Quelle Annotation und Übersetzung Deutsch/Englisch: OUDB Kazym Khanty Corpus, Text ID 1061, bearbeitet von Schön, Zsófia, URL: http://www.oudb.gwi.uni-muenchen. de/?cit=1061 (Zugriff vom 18.04.2017).

KK_text_007 = a:tet sumat. Die einsame Birke, Quelle: Молданов 1997a, 18, Quelle Annotation und Übersetzung Deutsch/Englisch: OUDB Kazym Khanty Corpus, Text ID 1058, bearbeitet von Schön, Zsófia, URL: http://www.oudb.gwi.uni-muenchen.de/?cit=1058 (Zugriff vom 18.04.2017).

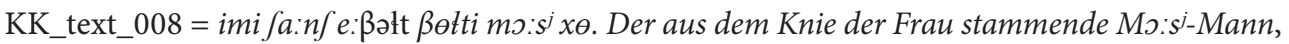
Quelle: Rédei 1968, 82-91, Quelle Annotation und Übersetzung Deutsch/Englisch: OUDB Kazym Khanty Corpus, Text ID 1117, bearbeitet von Schön, Zsófia, URL: http://www.oudb. gwi.uni-muenchen.de/?cit=1117 (Zugriff vom 18.04.2017).

KK_text_009 = jemay niot. Das heilige Kap, Quelle: Молданов 1997b, 49-52, Quelle Annotation und Übersetzung Deutsch/Englisch: OUDB Kazym Khanty Corpus, Text ID 1091, bearbeitet von Schön, Zsófia, URL: http://www.oudb.gwi.uni-muenchen.de/?cit=1091 (Zugriff vom 18.04.2017).

KK_text_010 = kat mo:si-ne, kat por-ne. Die zwei Mo:sj-Frauen, die zwei Por-Frauen, Quelle: Хомляк 2002, 18_28, Quelle Annotation und Übersetzung Deutsch/Englisch: OUDB Kazym Khanty Corpus, Text ID 1128, bearbeitet von Schön, Zsófia, URL: http://www.oudb.gwi.unimuenchen.de/?cit=1128 (Zugriff 18.04.2017).

NM_text_001 = mo:rtim ma:. Südland, Quelle: Černecov, Valerij Nikolajevič, »Как эква-пырись в страну мортим-ма летал« (Černecov-Archiv, Nr. 39, 184-190), in: Лукина (Hg.) 1990, 352-354, Quelle Annotation und Übersetzung Deutsch/Englisch: Chernetsov, V.: OUDB Northern Mansi Corpus, Text ID 889, bearbeitet von Janda, Gwen Eva, URL: http://www. oudb.gwi.uni-muenchen.de/?cit=889 (Zugriff vom 26.04.2017).

NM_text_002 = e: $k^{w} a$ piyris ${ }^{j} a: k^{w} e n t a l ~ o: l e \partial y . ~ a: k^{w} e$ witen minas. Wie E: $k^{w} a$ piyris ${ }^{j}$ um die Hand der

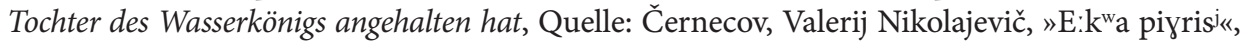


in: Unveröffentlichte Handschrift, Nr. 39, Text 23, Museum für Archäologie und Ethnographie Sibiriens der Staatlichen Universität Tomsk. Nach der Kopie des Ethnographischen Instituts der Ungarischen Akademie der Wissenschaften, Budapest, 167-174, Quelle Annotation und Übersetzung Deutsch/Englisch: Chernetsov, V.: OUDB Northern Mansi Corpus, Text ID 1238, bearbeitet von Janda, Gwen Eva, URL: http://www.oudb.gwi.uni-muenchen.de/?cit=1238 (Zugriff 26.04.2017).

NM_text_003 = ekwa piyrisj a ${ }^{w}$ ental oley. Wie Ekwa Piyrisj den Stadtfürsten hochnahm, Quelle: Černecov, Valerij Nikolajevič, »Как Эква Пырищ Усынг-отыра морочил (ČernecovArchiv, 40, Text 24 S. 223-239)«, in: Лукина/Чернецов (Hg.) 1935, 68-80, Quelle Annotation und Übersetzung Deutsch/Englisch: Chernetsov, V. 1935: OUDB Northern Mansi Corpus, Text ID 1234, bearbeitet von Janda, Gwen Eva, URL: http://www.oudb.gwi.uni-muenchen. de/?cit=1234 (Zugriff vom 26.04.2017).

NM_text_004 = pes porat o:jka xu:ram pi:y o:n'sij. In alten Zeiten hatte ein Mann drei Söhne,

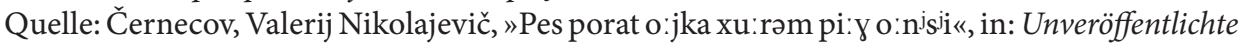
Handschrift, Nr. 2, Museum für Archäologie und Ethnographie Sibiriens der Staatlichen Universität Tomsk. Nach der Kopie des Ethnographischen Instituts der Ungarischen Akademie der Wissenschaften, Budapest, 3-15, Quelle Annotation und Übersetzung Deutsch/Englisch: Chernetsov, V. 1933: OUDB Northern Mansi Corpus, Text ID 1237, bearbeitet von Janda, Gwen Eva, URL: http://www.oudb.gwi.uni-muenchen.de/?cit=1237 (Zugriff vom 26.04.2017).

NM_text_005 = ji:wtili alyal ma:xum o:lmat. Rettung eines Mädchens durch ein Opfer, Quelle: Kannisto/Liimola (Hg.) 1951, 102-103, Quelle Annotation und Übersetzung Deutsch/Englisch: Kannisto \& Liimola 1951: OUDB Northern Mansi Corpus, Text ID 753, bearbeitet von Janda, Gwen Eva, URL: http://www.oudb.gwi.uni-muenchen.de/?cit=753 (Zugriff vom 26.04.2017).

NM_text_006 = owle:t mator porat. Die heilige Feuerflut, Quelle: Kannisto/Liimola (Hg.) 1951, 13f., Quelle Annotation und Übersetzung Deutsch/Englisch: Kannisto \& Liimola 1951: OUDB Northern Mansi Corpus, Text ID 742, bearbeitet von Janda, Gwen Eva, URL: http:// www.oudb.gwi.uni-muenchen.de/?cit=742 (Zugriff 26.04.2017).

NM_text_007 = sujpil lupta paฤxwit josay xum (mojt). Der Mann mit Skiern, von der Größe von Preiselbeerblättern (Märchen), Quelle: Алгадьева, Мария, "Sujpil lupta panxwit josan xum (mojt)«, in: Решетникова (Hg.) 2005, Quelle Annotation und Übersetzung Deutsch/Englisch: Luima Seripos 2005: OUDB Northern Mansi Corpus, Text ID 1073, bearbeitet von Janda, Gwen Eva, URL: http://www.oudb.gwi.uni-muenchen.de/?cit=1073 (Zugriff vom 26.04.2017).

NM_text_008 = kit jo:rn o:ley. janiy xumite... Der verbannte Samojede, Quelle: Kannisto/Liimola (Hg.) 1956, 9-20, Quelle Annotation und Übersetzung Deutsch/Englisch: Kannisto \& Liimola 1956: OUDB Northern Mansi Corpus, Text ID 1239, bearbeitet von Janda, Gwen Eva, URL: http://www.oudb.gwi.uni-muenchen.de/?cit=1239 (Zugriff vom 26.04.2017).

NM_text_009 = e: $k^{w} a$ pirris n $^{j} a: l$ pa:xwti. E: $k^{w} a$ piyris ${ }^{j}$ schießt mit seinem Pfeil, Quelle: Ромбандеева 1996, 15-22, Quelle Annotation und Übersetzung Deutsch/Englisch: Rombandeeva 1996: OUDB Northern Mansi Corpus. Text ID 1229, bearbeitet von Janda, Gwen Eva, URL: http://www.oudb.gwi.uni-muenchen.de/?cit=1229 (Zugriff vom 26.04.2017).

NM_text_010 = taytkotialiojka mir ats, lusamna jis, witjalpayana. Kriegszug des Alten von der Mittel-Sosva zum Wasserheiligtum, Quelle: Kannisto/Liimola (Hg.) 1951, 72-78, Quelle Annotation und Übersetzung Deutsch/Englisch: Kannisto \& Liimola 1951: OUDB Northern Mansi Corpus, Text ID 750, bearbeitet von Janda, Gwen Eva, URL: http://www.oudb.gwi. uni-muenchen.de/?cit=750 (Zugriff vom 26.04.2017).

NM_text_011 = nila xum mojt. A tale of four men, Quelle: Černecov, Valerij Nikolajevič, "Nila xum mojt «, in: Unveröffentlichte Handschrift, Nr. 39, Museum für Archäologie und Ethnographie Sibiriens der Staatlichen Universität Tomsk. Nach der Kopie des Ethnographischen Instituts der Ungarischen Akademie der Wissenschaften, Budapest, 195 Quelle Annotation und Übersetzung Deutsch/Englisch: Chernetsov, V.: OUDB Northern Mansi Corpus, Text ID 1232, bearbeitet von Janda, Gwen Eva, URL: http://www.oudb.gwi.uni-muenchen.de/?cit=1232 (Zugriff vom 26.04.2017). 
NM_text_012 = ekwa piyrisj (ak $\left.{ }^{w} e: k^{w} a t e\right) ~ a k^{w} e: n t a l$ o:lei:y. tot o:limate:n apiyrisje kon xajtiyli, The Old Woman's Nephew. Quelle: Černecov, Valerij Nikolajevič, »Как Эква-пырись на охоту ходил« (Černecov-Archiv, 44, 113-149), in: Лукина (Hg.) 1990, 366-373, Quelle Annotation und Übersetzung Deutsch/Englisch: Chernetsov, V. 1990: OUDB Northern Mansi Corpus, Text ID 1235, bearbeitet von Janda, Gwen Eva, URL: http://www.oudb.gwi.uni-muenchen. de/?cit=1235 (Zugriff vom 26.04.2017).

NM_text_013 = nusa e:kwa:y o:jka:y os karr. Die arme Alte, ihr Mann und der Specht, Quelle: Černecov, Valerij Nikolajevič, "Nusa ekway os ojkay«, in: Unveröffentlichte Handschrift, Nr. 40, Museum für Archäologie und Ethnographie Sibiriens der Staatlichen Universität Tomsk. Nach der Kopie des Ethnographischen Instituts der Ungarischen Akademie der Wissenschaften, Budapest, 181-183, Quelle Annotation und Übersetzung Deutsch/Englisch: Chernetsov, V.: OUDB Northern Mansi Corpus, Text ID 1233, bearbeitet von Janda, Gwen Eva, URL: http:// www.oudb.gwi.uni-muenchen.de/?cit=1233 (Zugriff vom 26.04.2017).

NM_text_014 = ak ${ }^{w}$ xum o:li takke:tpa:walt, takke:t u:sat. Steuererhebung der Schutzgeister, Quelle: Kannisto/Liimola (Hg.) 1951, 61-66, Quelle Annotation und Übersetzung Deutsch/Englisch: Kannisto \& Liimola: OUDB Northern Mansi Corpus, Text ID 745, bearbeitet von Janda, Gwen Eva, URL: http://www.oudb.gwi.uni-muenchen.de/?cit=745 (Zugriff vom 26.04.2017).

NM_text_015 = pe:tikoika kit piyaye jot kimna minasat. Die Entfernung eines Wurmes aus dem Ohr, Quelle: Kannisto/Liimola (Hg.) 1951, 106-107, Quelle Annotation und Übersetzung Deutsch/ Englisch: Kannisto \& Liimola 1951: OUDB Northern Mansi Corpus, Text ID 757, bearbeitet von Janda, Gwen Eva, URL: http://www.oudb.gwi.uni-muenchen.de/?cit=757 (Zugriff vom 26.04.2017).

NM_text_016 = E:li xo:n ma:t xan'isitaxtasuw. We Studied in a Faraway Country, Quelle: Mierova, Tamara, »E:li xo:n ma:t xanisistaxtasuw«, in: Решетникова (Hg.) 2005, Quelle Annotation und Übersetzung Deutsch/Englisch: Luima Seripos 2005: OUDB Northern Mansi Corpus, Text ID 1231, bearbeitet von Janda, Gwen Eva, URL: http://www.oudb.gwi.uni-muenchen. de/?cit=1231 (Zugriff vom 26.04.2017).

NM_text_017 = 7 maj - radiio xotal. May 7th - day of radio, Quelle: Сондыков, B.C. - Матвееван, Светлана, »7 maj - radijo xotal«, in: Решетникова (Hg.) 2005, Quelle Annotation und Übersetzung Deutsch/Englisch: Luima Seripos 2004: OUDB Northern Mansi Corpus, Text ID 1230, bearbeitet von Janda, Gwen Eva, URL: http://www.oudb.gwi.uni-muenchen. $\mathrm{de} /$ ?cit=1230 (Zugriff vom 26.04.2017).

SK_satz_0001 = mantayetna me: tomtam ttw sakkat. Bevor sie wegging, zog ich ihre Jacke an, Quelle: Schön, Zsófia, Postpositionale Datenbank, 2015, Quelle Annotation und Übersetzung Deutsch/Englisch: Zsófia Schön/Eigene Annotation.

SK_text_001=pi:tiankaliyan-o:piszyan A. The Little Bird and his Sister, Quelle: Csepregi 1998/2011, 66f., Quelle Annotation und Übersetzung Deutsch/Englisch: Csepregi, Márta 1998: OUDB Surgut Khanty Corpus, Text ID 732, bearbeitet von Schön, Zsófia, URL: http://www.oudb. gwi.uni-muenchen.de/?cit=732 (Zugriff vom 26.04.2017).

SK_text_002 = aj jayset. Three brothers, Quelle: Csepregi 1998/2011, 70-73, Quelle Annotation und Übersetzung Deutsch/Englisch: Csepregi, Márta 1998: OUDB Surgut Khanty Corpus, Text ID 734, bearbeitet von Schön, Zsófia, URL: http://www.oudb.gwi.uni-muenchen. $\mathrm{de} /$ ?cit=734 (Zugriff vom 26.04.2017).

SK_text_003 = ke:t i:mizan. The two women, Quelle: Csepregi 1998/2011, 74-81, Quelle Annotation und Übersetzung Deutsch/Englisch: Csepregi, Márta 1998: OUDB Surgut Khanty Corpus, Text ID 735, bearbeitet von Schön, Zsófia, URL: http://www.oudb.gwi.uni-muenchen. $\mathrm{de} /$ ?cit=735 (Zugriff vom 26.04.2017).

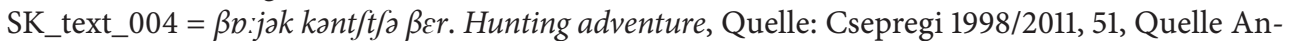
notation und Übersetzung Deutsch/Englisch: Csepregi, Márta 1998: OUDB Surgut Khanty Corpus, Text ID 728, bearbeitet von Schön, Zsófia, URL: http://www.oudb.gwi.uni-muenchen.de/?cit=728 (Zugriff vom 26.04.2017). 
SK_text_005 = pi:tiankalizan-o:piseyan B. The Little Bird and his Sister B, Quelle: Csepregi 1998/2011, 68-69, Quelle Annotation und Übersetzung Deutsch/Englisch: Kerezsi, Ágnes 1998: OUDB Surgut Khanty Corpus, Text ID 733, bearbeitet von Schön, Zsófia, URL: http:// www.oudb.gwi.uni-muenchen.de/?cit=733 (Zugriff vom 26.04.2017).

SK_text_006 = aßas ort pe:na ro:paltata qo: parili. Once upon a time there was a wealthy man, Quelle: Csepregi 1998/2011, 96-97, Quelle Annotation und Übersetzung Deutsch/Englisch: Csepregi, Márta 1998: OUDB Surgut Khanty Corpus, Text ID 737, bearbeitet von Schön, Zsófia, URL: http://www.oudb.gwi.uni-muenchen.de/?cit=737 (Zugriff vom 26.04.2017).

SK_text_007 = me: Battə ßeram. My life, Quelle: Csepregi 1998/2011, 56-59, Quelle Annotation und Übersetzung Deutsch/Englisch: Csepregi, Márta 1998: OUDB Surgut Khanty Corpus, Text ID 727, bearbeitet von Schön, Zsófia, URL: http://www.oudb.gwi.uni-muenchen. de/?cit=727 (Zugriff vom 26.04.2017).

SK_text_008 = 1996/A i:ki sesay Ber. 1996/A A man made a trap 1996/A, Quelle: Csepregi/Sosa 2009, Quelle Annotation und Übersetzung Deutsch/Englisch: Csepregi, Márta 2009: OUDB Surgut Khanty Corpus, Text ID 1081, bearbeitet von Schön, Zsófia, URL: http://www.oudb. gwi.uni-muenchen.de/?cit=1081 (Zugriff vom 26.04.2017).

SK_text_009 = 2008/A i:ki sesay Ber. 2008/A A man made a trap 2008/A, Quelle: Csepregi/Sosa 2009, Quelle Annotation und Übersetzung Deutsch/Englisch: Sosa, Sachiko 2009: OUDB Surgut Khanty Corpus, Text ID 1085, bearbeitet von Schön, Zsófia, URL: http://www.oudb. gwi.uni-muenchen.de/?cit=1085 (Zugriff vom 26.04.2017).

SK_text_010 = 1996/C ke:ßiza manam i:mi. 1996/C The woman who changed into a cuckoo 1996/C, Quelle: Csepregi/Sosa 2009, Quelle Annotation und Übersetzung Deutsch/Englisch: Csepregi, Márta 2009: OUDB Surgut Khanty Corpus, Text ID 1084, bearbeitet von Schön, Zsófia, URL: http://www.oudb.gwi.uni-muenchen.de/?cit=1084 (Zugriff vom 26.04.2017).

SK_text_011 = 2008/C ke:Biya manam i:mi. 2008/C The woman who changed into a cuckoo 2008/C, Quelle: Csepregi/Sosa 2009, Quelle Annotation und Übersetzung Deutsch/Englisch: Sosa, Sachiko 2009: OUDB Surgut Khanty Corpus, Text ID 1087, bearbeitet von Schön, Zsófia, URL: http://www.oudb.gwi.uni-muenchen.de/?cit=1087 (Zugriff vom 26.04.2017).

SK_text_012 = pe: qp:n mәуәэ mat ne:. Our guest, Quelle: Каюкова 2012, Quelle Annotation und Übersetzung Deutsch/Englisch: Wissenschaftliche Übung Surgut-Ostjakisch 2, LMU, SoSe 2013 (bearbeitet von Zsófia Schön, Stefanie Eckman, Corinna Nierderreiter, Mia Klee und Aleksander Wiatr).

SK_text_013 = pi:tiankali pe:nəo:pi ßaltəyən. The Little Bird and his Sister, Quelle: Csepregi 1993, Quelle Annotation: Wissenschaftliche Übung Surgut-Chantisch, LMU, WiSe 2012/2013 (bearbeitet von Stefanie Eckman), Übersetzung Deutsch: Stefanie Eckmann (Wissenschaftliche Übung Surgut-Chantisch, LMU, WiSe 2012/2013), Übersetzung Englisch: Clive Tolley.

\section{Sekundärliteratur}

Abondolo 1998a = Abondolo, Daniel, "Hungarian«, in: Abondolo, Daniel (Hg.), The Uralic Languages, London/New York 1998, 428-456.

Abondolo 1998b = Abondolo, Daniel, »Introduction«, in: Abondolo, Daniel (Hg.), The Uralic Languages, London/New York 1998, 1-42.

Abondolo 1998c = Abondolo, Daniel, »Khanty«, in: Abondolo, Daniel (Hg.), The Uralic Languages, London/New York 1998, 358-386.

Abondolo (Hg.) 1998 = Abondolo, Daniel (Hg.), The Uralic Languages, London/New York 1998.

Ahlqvist 1894 = Ahlqvist, August, Wogulische Sprachtexte nebst Entwurf einer wogulischen Grammatik (Mémoires de la Société Finno-Ougrienne, 7), Helsinki 1894. 
Aikhenvald/Dixon (Hg.) 2012 = Aikhenvald, Alexandra Y./Dixon, R[obert] M.W. (Hg.), Possession and Ownership, Oxford 2012.

Arany/Gyulai (Hg.) 1872 = Arany, László/Gyulai, Pál (Hg.), Csongrádmegyei gyüjtés. Magyar népköltési gyüjtemény, új folyam, Pest 1872, URL: http://www.gutenberg.org/files/43324/43324h/43324-h.htm (Zugriff vom 17.04.2017).

Ariel 1999 = Ariel, Mira, »Accessibility theory: An overview», in: Sanders, Ted/Schilperoord, Joost/Spooren, Wilbert (Hg.), Text representation (Human cognitive processing series), Amsterdam/Philadelphia 1999, 29-87.

Баландин/Вахрушева (Нg.) 1958 = Баландин, А.Н./Вахрушева, М.П. [Balandin A.N./ Vahruševa, М.P.] (Нg.), Мансийско-русский словарь с лексическими параллелями из южно-мансийского (кондинского) диалекта, St. Petersburg 1958, URL: http://babel.gwi.uni-muenchen.de/media/ downloads/Balandin_Vahrusheva_mansi-russ_sl.pdf (Zugriff vom 12.08.2016).

Bally 1926 = Bally, Charles, Le langage et la vie, Paris 1926.

Barker 1995 = Barker, Chris, Possessive descriptions, Preprint, Stanford 1995, URL: https://www. nyu.edu/projects/barker/barker-dissertation.pdf (Zugriff vom 20.03.2017).

Barker 2008 = Barker, Chris, »Possessives and relational nouns«, in: Maienborn, Claudia/Heusinger, Klaus von/Portner, Paul (Hg.), Semantics: An International Handbook of Natural Language Meaning, Berlin 2008, 1-27, URL: http://semanticsarchive.net/Archive/WYxOTc5M/ barker-possessives.pdf (Zugriff vom 08.10.2015).

Benkö/Samu (Hg.) 1972 = Benkö, Loránd/Samu, Imre (Hg.), The Hungarian Language (Janua Linguarum. Series Practica, 134), The Hague/Budapest 1972.

Bickel/Nichols 2013 = Bickel, Balthasar/Nichols, Johanna, "Obligatory Possessive Inflection «, in: Dryer, Matthew S./Haspelmath, Martin (Hg.), The World Atlas of Language Structures Online, Leipzig 2013, URL: http://wals.info/chapter/58 (Zugriff vom 27.01.2014).

Biermann 1985 = Biermann, Anna, Possession und Zuschreibung im Ungarischen (Continuum, 4), Tübingen 1985.

Bögel/Butt 2013 = Bögel, Tina/Butt, Miriam, »Possessive clitics and ezafe in Urdu«, in: Börjars, Kerstin/Denison, David/Scott, Alan (Hg.), Morphosyntactic Categories and the Expression of Possession (Linguistik Aktuell/Linguistics Today, 199), Amsterdam/Philadelphia 2013, 291-322.

Börjars/Denison/Scott (Hg.) 2013 = Börjars, Kerstin/Denison, David/Scott, Alan (Hg.), Morphosyntactic Categories and the Expression of Possession (Linguistik Aktuell/Linguistics Today, 199), Amsterdam/Philadelphia 2013.

Bosch 1983 = Bosch, Peter, Agreement and Anaphora A Study of the Role of Pronouns in Syntax and Discourse, London 1983.

Brinker 2005 = Brinker, Klaus, Linguistische Textanalyse Eine Einführung in Grundbegriffe und Methoden, Berlin 2005.

Bühler [1934] 1982 = Bühler, Karl, Sprachtheorie: Die Darstellungsfunktion der Sprache. Mit einem Geleitwort von Friedrich Kainz (UTB für Wissenschaft, 1159), Neudruck der Ausgabe von 1934, Stuttgart/New York 1982.

Bußmann 2002 = Bußmann, Hadumod, Lexikon der Sprachwissenschaft, Stuttgart 2002.

Cassirer [1926] 1977 = Cassirer, Ernst, Philosophie der symbolischen Formen, 3 Bde., 7. Aufl., Darmstadt 1977.

Collinder 1957 = Collinder, Björn, A Handbook of the Uralic languages, Part 2: Survey of the Uralic languages, Stockholm 1957.

Collinder 1960 = Collinder, Björn, A Handbook of the Uralic languages, Part 3 Comparative grammar of the Uralic languages, Stockholm 1960.

Comrie 1975 = Comrie, Bernard, »Subjects and Direct Objects in Uralic languages: a functional explanation of case-marking systems", in: Études Finno-Ougriennes XII (1975), 5-17.

Comrie 1988 = Comrie, Bernard, »General features of the Uralic languages «, in: Sinor, Denis (Hg.), The Uralic Languages. Description, History and Foreign Influences (Handbuch der Orientalistik, 1), Leiden/New York/Kopenhagen 1988, 451-477. 
Consten 2004 = Consten, Manfred, Anaphorisch oder deiktisch? Zu einem integrativen Modell domänengebundener Referenz (Linguistische Arbeiten, 484), Tübingen 2004.

Croft 2001 = Croft, William, Radical construction grammar, Oxford 2001.

Csepregi 1993 = Csepregi, Márta, "Lintunen ja hänen sisarensa. Hantilainen satu Tromagan-joen varrelta«, in: Kulonen, Ulla-Maija (Hg.) Festschrift für Raija Bartens zum 25.10.1993 (Mémoires de la Société Finno-Ougrienne, 215), Helsinki 1993, 49-52.

Csepregi 1998 = Csepregi, Márta, Finnugor kalauz, Budapest, 1998.

Csepregi 1998/2011 = Csepregi, Márta, Szurguti Osztják Chrestomathia (Studia Uralo-Altaica. Supplementum, 6), Szeged 1998/2011, URL: http://www.babel.gwi.uni-muenchen.de/media/ downloads/SzOCh_FUT_20110721.pdf (Zugriff vom 29.11.2012).

Csepregi/Sosa 2009 = Csepregi, Márta/Sosa, Sachiko, "Comparable sample texts of Surgut Khanty in 1996 and 2008«, in: Journal de la Société Finno-Ougrienne 92 (2009), 193-208, URL: http://www.sgr.fi/susa/92/csepregisosa.pdf (Zugriff vom 17.04.2017).

Cysouw 2007 = Cysouw, Michael, »Building semantic maps: the case of person marking «, in: Wälchli, Bernhard/Miestamo, Matti (Hg.), New challenges in Typology. Broadening the Horizons and Redefining the Foundations (Trends in Linguisitics Studies and Monographs, 189), Berlin/New York 2007, 225-249.

Daneš 1970 = Daneš, František, »Zur linguistischen Analyse der Textstruktur«, in: Folia Linguistica 4 (1970), 72-78.

Décsy 1965 = Décsy, Gyula, Einführung in die finnisch-ugrische Sprachwisssenschaft, Wiesbaden 1965.

DeHaan 2004 = DeHaan, Ferdinand, On representing semantic maps, [2004], URL: http://citeseerx. ist.psu.edu/viewdoc/download?doi=10.11.163.6640\&rep=rep1\&type=pdf (Zugriff vom 04.11.2011).

Den Dikken 2015 = Den Dikken, Marcel, » The morphosyntax of (in)alienably possessed noun phrases. The Hungarian contribution", in: Kiss, Katalin É. [u.a] (Hg.), Approaches to Hungarian, 14. Papers from the 2013 Piliscsaba Conference, Amsterdam/Philadelphia 2015, 121-145.

Diessel 2016 = Diessel, Holger, Demonstratives, Joint Attention, and the Emergence of Grammar", in: Cognitive Linguistics 17/4 (2006), 463-489, URL: http://www.personal.uni-jena. de/ x4diho/Demonstratives, \%20joint\%20attention,\%20and\%20the $\% 20$ emergence $\% 20$ of\%20grammar.pdf (Zugriff vom 14.06.2013).

Dik 1997a = Dik, Simon C., The theory of Functional Grammar, Part 1: The structure of the clause, Berlin/New York 1997.

Dik 1997b = Dik, Simon C., The theory of Functional Grammar, Part 2: Complex and derived constructions, Berlin/New York 1997.

Drosdowski (Hg.) 1996 = Drosdowski, Günther (Hg.), Duden Rechtschreibung der deutschen Sprache, Mannheim/Leipzig/Wien 1996.

Dryer 2013 = Dryer, Matthew S., »Position of Pronominal Possessive Affixes «, in: Dryer, Matthew S./Haspelmath, Martin (Hg.), The World Atlas of Language Structures Online, Leipzig 2013, URL: http://wals.info/chapter/57 (Zugriff vom 27.01.2014).

Dryer/Haspelmath (Hg.) 2013 = Dryer, Matthew S./Haspelmath, Martin (Hg.), The World Atlas of Language Structures Online, Leipzig 2013, URL: http://wals.info/ (Zugriff vom 20.04.2015).

Edygarova 2009 = Edygarova, Svetlana, »Attributive Possession in Udmurt Language«, in: Linguistica Uralica XLV/2 (2009), 101-118, DOI: http://dx.doi.org/10.3176/lu.2009.2.02 (Zugriff vom 20.09.2017).

Edygarova 2013 = Edygarova, Svetlana, Possessive Suffixes as Referential Devices in Udmurt (Talk Grammar\&Context), Tartu 2013.

Ehlich 1982 = Ehlich, Konrad, »Anaphora and Deixis: same, similar or different? «, in: Jarvella, Robert J./Klein, Wolfgang (Hg.), Speech, Place and Action. Studies of Deixis and Related Topics, Chichester/New York 1982, 315-338.

Elsen 2014 = Elsen, Hilke, Linguistische Theorien, Tübingen 2014 .

Erkü/Gundel 1987 = Erkü, Feride/Gundel, Jeanette, »The Pragmatics of Indirect Anaphors «, in: Verschueren, J./Bertuccelli-Papi, Marcella (Hg.), The Pragmatic Perspective. Selected Papers 
from the 1985 Internat. Pragmatics Conference (Pragmatics \& Beyond Companion Series, 5), Amsterdam/Philadelphia 1987, 533-545.

Filchenko 2007 = Filchenko, Andrey Yury, A grammar of Eastern Khanty, Houston, Texas 2007, URL: http://babel.gwi.uni-muenchen.de/media/downloads/Filtchenko_2007_Diss-GrammarEasternKhanty.pdf (Zugriff vom 29.11.2012).

Filchenko 2014 = Filchenko, A., "Possessive constructions in Eastern Khanty«, in: Томский журнал ЛИНГ и АНТР/Tomsk Journal LING \& ANTHRO 1/3 (2014), 83-95, URL: http://ling.tspu. edu.ru/files/ling/PDF/articles/filchenko_a._y._83_95_1_3_2014.pdf (Zugriff vom 09.05.2015).

Fix 2014 = Fix, Ulla, »Text und Textlinguistik«, in: Janich, Nina (Hg.), Textlinguistik, 15 Einführungen (Narr Studienbücher), Tübingen 2014, 15-34.

Forgács 2007 = Forgács, Tamás, Ungarische Grammatik, 3, Wien 2007.

Fox 1996 = Fox, Barbara, Discourse Structure and Anaphora, Cambridge 1996.

Fraurud 2001 = Fraurud, Kari, »Possessives with extensive use: A source of definite articles? «, in: Baron, Irène/Herslund, Michael/Sørensen, Finn (Hg.), Dimensions of possession (Typological Studies in Language, 47), Amsterdam/Philadelphia 2001, 243-267.

Freeze 1992 = Freeze, Ray, »Existentials and other locatives«, in: Language. Journal of the Linguistic Society of America 68/3 (1992), 553-593.

Gansel/Jürgens 2002 = Gansel, Christina/Jürgens, Frank, Textlinguistik und Textgrammatik, Eine Einführung, Wiesbaden 2002.

Gerland 2011 = Gerland, Doris, From possessive suffixes to definite articles, Workshop on Languages with and without Articles, Universität Düsseldorf, Düsseldorf 2011, URL: http://archive.sfl.cnrs. fr/sites/sfl/IMG/pdf/lsalaallgerlandHO.pdf (Zugriff vom 24.05.2017).

Gerland 2014a = Gerland, Doris, "Definitely Not Possessed? Possessive Suffixes with Definiteness Marking Function", in: Gamerschlag, Thomas [u.a.] (Hg.), Frames and Concept Types. Applications in Language and Philosophy (Studies in Linguistics and Philosophy, 94), Cham/ Heidelberg 2014, 269-292.

Gerland 2014b = Gerland, Doris, "Definiteness in Uralic: Where does it come from? Abstract«, in: Головко, E.В. (Hg.), Системные изменения в языках россии. Тезисы докладов международной научной конференции Санкт-Петербург, 16-18 октября 2014 г., St. Petersburg 2014, 85-90, URL: http://iling.spb.ru/confs/syschanges2014/materials.pdf (Zugriff vom 24.05.2017).

Givón 1983 = Givón, Talmy, Topic Continuity in Discourse: A Quantitative Cross-Language Study, Amsterdam/Philadelphia 1983.

Givón 2001a = Givón, Talmy, Syntax, I, Amsterdam/Philadelphia 2001.

Givón 2001b = Givón, Talmy, Syntax, An Introduction, II, Amsterdam/Philadelphia 2001.

Goldberg 1995 = Goldberg, Adele E., Constructions. A construction grammar approach to argument structure, Chicago 1995.

Grenoble 2006 = Grenoble, Lenore A., »Discourse Analysis«, in: Glossos 8 (2006), 1-35, URL: https://slaviccenters.duke.edu/sites/slaviccenters.duke.edu/files/media_items_files/ 8grenoble.original.pdf (Zugriff vom 12.12.2011).

Gulya 1966 = Gulya, János, Eastern Ostyak Chrestomathy (Uralic and Altaic Series, 51), Bloomington/The Hague 1966, URL: http://babel.gwi.uni-muenchen.de/media/downloads/ Gulya_EasternOstyakChrestomathy.pdf (Zugriff vom 30.11.2012).

Gundel/Hinds 1985 = Gundel, Jeanette K./Hinds, John, »Introduction: Special issue on $>$ shared knowledge «, in: Journal of Pragmatics 9/1 (1985), 5f., URL: http://www.sciencedirect.com. emedien.ub.uni-muenchen.de/science/article/pii/0378216685900499/pdf?md5=6f091688b92 cfa39088c0599b2d00cce\&pid=1-s2.0-0378216685900499-main.pdf (Zugriff vom 04.10.2015).

Hajdú/Domokos (Hg.) 1987 = Hajdú, Péter/Domokos, Péter (Hg.), Die uralischen Sprachen und Literaturen, Hamburg 1987.

Halász/Földes/Uzonyi (Hg.) (2006) = Halász, Elöd/Földes, Csaba/Uzonyi, Pál (Hg.), Magyarnémet nagyszótár, Budapest 2006. 
Hansen 1998 = Hansen, Maj-Britt Moosegard, „Cohesion and Cohherence«, in: Hansen, MajBritt Moosegard, The function of discourse particles: A study with special reference to spoken Standard French, Amsterdam/Philadelphia 1998, 171-199.

Haspelmath 1999 = Haspelmath, Martin, "External possession in a European areal perspective«, in: Payne, Doris L./Barshi, Immanuel (Hg.), External possession (Typological Studies in Language, 39), Amsterdam/Philadelphia 1999, 109-135.

Haspelmath 2008 = Haspelmath, Martin, "Framework-free grammatical theory «, in: Heine, Bern/Narrog, Heiko (Hg.), The Oxford Handbook of Language Typology, Oxford 2008, 341-365.

Haspelmath/Sims 2010 = Haspelmath, Martin/Sims, Andrea D., Understanding morphology, 2, London 2010.

Hawkins 1978 = Hawkins, John, Definiteness and Indefiniteness: A Study in Reference and Grammaticality Prediction, London 1978.

Heine 1997 = Heine, Bernd, Possession, Cognitive Forces and Grammaticalization (Cambridge Studies in Linguistics, 83), Cambridge 1997.

Heinemann 2008 = Heinemann, Wolfgang, »Textpragmatische und kommunikative Ansätze«, in: Janich, Nina (Hg.), Textlinguistik. 15 Einführungen (Narr Studienbücher), Tübingen 2008, 115-143.

Hellwig 1984 = Hellwig, Peter, "Grundzüge einer Theorie des Textzusammenhangs", in: Rothkegel, Annely/Sandig, Barbara (Hg.), Text - Textsorten - Semantik. Linguistische Modelle und maschinelle Verfahren (Papiere zur Textlinguistik, 52), Hamburg 1984, 51-79.

Hengeveld/Mackenzie 2008 = Hengeveld, Kees/Mackenzie, J. Lachlan, Functional Discourse Grammar. A typologically - based theory of language structure, Oxford 2008.

Hessky (Hg.) 2002 = Hessky, Regina (Hg.), Magyar-Német Kéziszótár Ungarisch-Deutsches Handwörterbuch, Szeged 2002.

Хомляк 2002 = Хомляк, Л. Р., Арєм-моньщєм ел ки мӑнлу. Если моя песня-сказка дальше пойдёт. Фольклорное творчество Пелагеи Алексеевны Гришкиной из деревни Тугияны, Chanty-Mansijsk 2002.

Honti 1978 = Honti, László, "Az osztják személyjelölő szuffixumok történeti áttekintése«, in: Nyelvtudományi Közlemények 78 (1978), 71-119.

Honti 1983 = Honti, László, »A harmadik személyt jelölő szuffixumok a vogulban«, in: Nyelvtudományi Közlemények 85 (1983), 349-355.

Honti 1984 = Honti, László, Chrestomathia Ostiacica, Budapest 1984, URL: http://babel.gwi.unimuenchen.de/media/downloads/Honti_Chrestomathia_Ostiacica_grammar.pdf (Zugriff vom 30.11.2012).

Honti 1988a = Honti, László, "Die ostjakische Sprache«, in: Sinor, Denis (Hg.), The Uralic Languages. Description, History and Foreign Influences (Handbuch der Orientalistik), Leiden/New York/Kopenhagen 1988, 172-196.

Honti 1988b = Honti, László, »Die wogulische Sprache«, in: Sinor, Denis (Hg.), The Uralic Languages. Description, History and Foreign Influences (Handbuch der Orientalistik), Leiden/New York/Kopenhagen 1988, 147-171.

Honti 1998 = Honti, László, »ObUgrian«, in: Abondolo, Daniel (Hg.), The Uralic Languages, London/New York 1998, 327-357.

Honti 2004 = Honti, László, Uráli birtokos szerkezetek (Vortrag vom 06.12.2004), Budapest 2004, URL: http://www.mta.hu/fileadmin/szekfoglalok/000302.pdf (Zugriff vom 23.07.2015).

Honti 2005 = Honti, László, »A magyar habitív és izafetszerkezet történeti hátteréről«, in: Magyar Nyelv 101/4 (2005), 449-454, URL: http://epa.oszk.hu/00000/00032/00027/pdf/hontil.pdf (Zugriff vom 10.04.2012).

Honti 2006 = Honti, László, »On the historical background of habitive and izafet constructions in Hungarian «, in: Acta Linguistica Hungarica 53/1 (2006), 41-51.

Honti 2007a = Honti, László, »A birtoklás kifejezésének eszközei az uráli nyelvekben szinkrón és diakrón szempontból«, in: Nyelvtudományi Közlemények 104 (2007), 7-56, URL: http://www. nytud.hu/nyk/nyk104.pdf (Zugriff vom 06.07.2015). 
Honti 2007b = Honti, László, "Syntaktische Konstruktionen fremden Ursprungs. Eine skeptische Stellungnahme«, in: Incontri Linguistici 30 (2007), 49-70.

Honti 2008 = Honti, László, »Mi az izafet? (Egy kis búvárkodás a terminológiai zavarosban)«, in: Bereczki, András/Csepregi, Márta/Klima, László (Hg.), Ünnepi írások Havas Ferenc tiszteletére (Uralisztikai Tanulmányok), Budapest 2008, 291-311.

Honti 2012 = Honti, László, »Was ist Izafet?: Eine kleine Forschungsreise durch die terminologische Trübe«, in: Orioles, Vincenzo (Hg.), Per Roberto Gusmani: studi in ricordo: 2 (Linguistica storica e teorica, 1), Udine 2012, 181-197.

IPA (Hg.) 1999 = International Phonetic Association (Hg.), Handbook of the international phonetic association. A guide to the use of the international phonetic alphabet, Cambridge 1999.

Jakobson/Halle 1956 = Jakobson, Roman/Halle, Morris, Fundamentals of language, The Hague 1956, Permalink: http://hdl.handle.net/11858/00-001M-0000-002B-AE53-D (Zugriff vom 28.06.2018).

Janda 2006 = Janda, Laura A., »Cognitive Linguistics«, in: Glossos 8 (2006), 1-60, URL: https:// slaviccenters.duke.edu/sites/slaviccenters.duke.edu/files/media_items_files/8janda.original.pdf (Zugriff vom 12.12.2011).

Janda 2009 = Janda, Laura A., »What is the role of semantic maps in cognitive linguistics? «, in: Stalmaszczyk, Piotr/Wieslaw, Oleksy (Hg.), Cognitive approaches to language and linguistic data. Studies in honor of Barbara Lewandowska-Tomaszczyk, Frankfurt a.M. 2009, 105.

Janda 2001 = Janda, Gwen Eva, Funktionen der Possessivmarker in den ugrischen Sprachen (Hausarbeit zur Erlangung des akademischen Grades Magister Artium, unveröffentlichtes Manuskript), Ludwig-Maximilians-Universität München 2011.

Janda 2015 = Janda, Gwen Eva, "Northern Mansi possessive suffixes in non-possessive function ", in: Eesti ja soome-ugri keeleteaduse ajakiri. Journal of Estonian and Finno-Ugric Linguistics 6/2 (2015) [Special issue on Referential devices in Uralic languages], 243-258, DOI: http://dx.doi. org/10.12697/jeful.2015.6.2.10 (Zugriff vom 12.04.2017).

Каюкова 2012 = Каюкова, Людмила, »Ре: qр:n mәуәу mat $\int$ ne:«, in: Ханты Ясенг 22 (2012).

Kálmán 1965 = Kálmán, Béla, Vogul Chrestomathy (Uralic and Altaic series, 46), Bloomington/ The Hague 1965.

Kálmán 1963 = Kálmán, Béla, Chrestomathia Vogulica, Budapest 1963.

Kangasmaa-Minn 1984 = Kangasmaa-Minn, Eeva, »On the Possessive Constructions in Finno-Ugric«, in: Nyelvtudomány Közlemények 86 (1984), 118-123.

Kannisto/Liimola (Hg.) 1951 = Kannisto, Artturi/Liimola, Matti, Wogulische Volksdichtung gesammelt und übersetzt von Artturi Kannisto, bearbeitet und herausgegeben von Matti Liimola, I Texte mythischen Inhalts (Mémoires de la Société Finno-Ougrienne, 101), Helsinki 1951.

Kannisto/Liimola (Hg.) 1956 = Kannisto, Artturi/Liimola, Matti, Wogulische Volksdichtung gesammelt und übersetzt von Artturi Kannisto, bearbeitet und herausgegeben von Matti Liimola, III Märchen, (Mémoires de la Société Finno-Ougrienne, 111), Helsinki 1956.

Решетникова (Нg.) 2005 = Решетникова, Раиса (Hg.), Луйма сэрйос, Chanty-Mansijsk 2005.

Kay/Zimmer 1976 = Kay, Paul/Zimmer, Karl, »On the semantics of compounds and genitives in English «, in: Proceedings of the Sixth California Linguistics Association Conference, San Diego 1976, 29-35.

Kenesei/Vago/Fenyvesi (Hg.) 1998 = Kenesei, István/Vago, Robert M./Fenyvesi, Anna (Hg.), Hungarian. Descriptive grammars, London 1998.

Keresztes 1998 = Keresztes, László, »Mansi«, in: Abondolo, Daniel (Hg.), The Uralic Languages, London/New York 1998, 387-427.

Kern $2010=$ Kern, Beate, Metonymie und Diskurskontinuität im Französischen (Linguistische Arbeiten, 531), Berlin/New York 2010.

Keszler/Lengyel 2008 = Keszler, Borbála/Lengyel, Klára, Ungarische Grammatik, Hamburg 2008. Kibrik 2001 = Kibrik, Andrej A., »Cognitive discourse analysis: Some results «, in: Németh, Enikő T. (Hg.), Cognition in Language Use. Selected papers from the 7th International Pragmatics 
Conference 1, Antwerpen 2001, 164-180, URL: http://www.philol.msu.ru/\%7Eotipl/new/ main/people/kibrik-aa/files/Cognitive_DA@IPA_2001.pdf (Zugriff vom 03.10.2012).

Klumpp 2013 = Klumpp, Gerson, Four Functions of Komi Possessive Suffixes (Plenary Talk Grammar\&Context Tartu), Tartu 2013.

Körtvély 2009 = Körtvély, Erika, »A tundra nyenyec birtokos személyragok funkcióról«, in: Fancsaly, Éva (Hg.), Tanár és tanítvány: írások Györke József és Hajdú Péter tiszteletére 2002-2007, Budapest/Pécs 2009, 77-92.

Kolde 1989 = Kolde, Gottfried, Der Artikel in deutschen Sachverhaltsnominalen (Reihe germanistische Linguistik, 96), Tübingen 1989.

Koptjevskaja-Tamm 2001 = Koptjevskaja-Tamm, Maria, »Adnominal possession «, in: Haspelmath, M. [u.a.] (Hg.), Language typology and language universals. An international handbook, 2, Berlin 2001, 960-970.

Koptjevskaja-Tamm 2002 = Koptjevskaja-Tamm, Maria, »Adnominal possession in the European languages: form and function«, in: Sprachtypologie und Universalienforschung 55/2 (2002), 141-172.

Koptjevskaja-Tamm 2003 = Koptjevskaja-Tamm, Maria, »Possessive noun phrases in the languages of Europe«, in: Plank, Frans (Hg.), Noun phrase structure in the languages of Europe, Berlin 2003, 621-722.

Kornai 1985 = Kornai, András, »The internal structure of noun phrases«, in: Kenesei, István (Hg.), Approaches to Hungarian. One. Data and Descriptions, Szeged 1985, 79-92.

Kovgan 2005 = Kovgan, Elena, »The textual structure of Khanty/co-reference and anaphora", in: Fernandez-Vest, Jocelyne M.M. (Hg.), Les langues ouraliennes aujourd'hui. Approche linguistique et cognitive. The Uralic languages today. A linguistic and cognitive approach, Paris 2005, 547-560.

Kovgan 2001 = Kovgan, Elena V., »Reference-tracking in Khanty«, in: Seilenthal, Tõnu (Hg.), Congressus Nonus Internationalis Fenno-Ugristarum 7.-13.8.2000, Tartu. Pars V, Tartu 2001, 145-153.

Krifka 2008 = Krifka, Manfred, »Basic Notions of Information Structure«, in: Acta Linguistica Hungarica 55/3-4 (2008), 243-276, DOI: http://dx.doi.org/10.1556/ALing.55.2008.3-4.2 (Zugriff vom 05.06.2012).

Kulonen 2007 = Kulonen, Ulla-Maija, Itämansin kielioppi ja tekstejä (Hilfsmittel für das Studium der finnisch-ugrischen Sprachen, XV), Helsinki 2007, URL: http://babel.gwi.uni-muenchen.de/media/downloads/grammar/EasternMansi/EasternMansiGrammar.pdf (Zugriff vom 09.09.2013).

Künnap 2004 = Künnap, Ago, »About the non-personal definite function of the Uralic 3rd person possessive suffix «, in: Linguistica Uralica XL 1 (2004), 1-4.

Kunze (Hg.) 1999 = Kunze, Christina (Hg.), Modern magyar novellák. Moderne Ungarische Erzählungen, München 1999.

Laanest 1982 = Laanest, Arvo, Einführung in die ostseefinnischen Sprachen, Hamburg 1982.

Lambrecht 1994a = Lambrecht, Knud, Information structure and sentence form, Cambridge 1994.

Lambrecht 1994b = Lambrecht, Knud, Topic, focus, and the mental representations of discourse referents, Cambridge 1994.

Langacker 1993 = Langacker, Ronald W., »Reference-point constructions«, in: Cognitive Linguistics 4/1 (1993), 1-38, DOI: https://doi.org/10.1515/cogl.1993.4.1.1 (Zugriff vom 19.10.2018).

Langacker 1995 = Langacker, Ronald W., »Possession and possessive constructions", in: Taylor, John R./MacLayry Robert E. (Hg.), Language and the cognitive construal of the world (Trends in Lingustics, Studies and Monographs, 82), Berlin/New York 1995, 51-79.

Langacker 2008 = Langacker, Ronald W., »Chapter 5. Cognitive Grammar «, in: Heine Bernd/ Narrog, Heiko (Hg.), The Oxford Handbook of Language Typology, Oxford 2008, 87-109.

Lehmann 2013a = Lehmann, Christian, Anapher, [2013], URL: http://www.christianlehmann. eu/ling/lg_system/sem/index.html?http://www.christianlehmann.eu/ling/lg_system/sem/ anapher.html (Zugriff vom 16.06.2015).

Lehmann 2013b = Lehmann, Christian, Deixis, [2013], URL: http://www.christianlehmann.eu/ ling/lg_system/grammar/morph_syn/index.html?http://www.christianlehmann.eu/ling/ lg_system/grammar/morph_syn/deixis.php (Zugriff vom 24.05.2017). 
Lehmann 2013c = Lehmann, Christian, Onomasiologie und Semasiologie, [2013], URL: http:// www.christianlehmann.eu/termini/index.html?http://www.christianlehmann.eu/termini/ onomasiologie\&semasiologie.html (Zugriff vom 03.02.2015).

Leinonen 2006 = Leinonen, Marja, »Omistussuhteen ulokkeita: komin possessiivisuffiksin ei-possessiivisista funktioista«, in: Journal de la Société Finno-Ougrienne 91 (2006), URL: http://www.sgr.fi/susa/91/leinonen.pdf (Zugriff vom 21.03.2012).

Lemnitzer/Zinsmeister 2010 = Lemnitzer, Lothar/Zinsmeister, Heike, Korpuslinguistik. Eine Einführung, (Narr Studienbücher), Tübingen, 2010.

Lévy-Bruhl 1914 = Lévy-Bruhl, Lucien, »L'expression de la possession dans les langues mélanésiennes«, in: Mémoires de la Société de Linguistique de Paris 19 (1914/16), 96-104.

Li (Hg.) $1976=\mathrm{Li}$, Charles N. (Hg.), Subject and Topic, New York 1976.

Löbner 2011 = Löbner, Sebastian, »Concept types and determination «, in: Journal of Semantics 28/3 (2011), 279-333.

Loos (Hg.) 2004a = Loos, Eugene E. [u.a.] (Hg.), »What is a nonfinite verb«, in: Glossary Of Linguistic Terms, (2004), URL: http://www-01.sil.org/linguistics/GlossaryOflinguisticTerms/ WhatIsANonfiniteVerb.htm (Zugriff vom 22.08.2015).

Loos (Hg.) 2004b = Loos, Eugene E. [u.a.] (Hg.), »What is a noun«, in: Glossary Of Linguistic Terms, (2004), URL: http://www-01.sil.org/linguistics/GlossaryOflinguisticTerms/WhatIsANoun.htm (Zugriff vom 22.08.2015).

Loos (Hg.) 2004c = Loos, Eugene E. [u.a.] (Hg.), »What is a proper noun«, in: Glossary Of Linguistic Terms, (2004), URL: http://www-01.sil.org/linguistics/GlossaryOflinguisticTerms/ WhatIsAProperNoun.htm (Zugriff vom 22.08.2015).

Loos (Hg.) 2004d = Loos, Eugene E. [u.a.] (Hg.), »What is a verbal noun«, in: Glossary Of Linguistic Terms, (2004), URL: http://www-01.sil.org/linguistics/GlossaryOflinguisticTerms/ WhatIsAVerbalNoun.htm (Zugriff vom 11.07.2015).

Loos (Hg.) 2004e = Loos, Eugene E. [u.a.] (Hg.), "What is definite identifiability», in: Glossary Of Linguistic Terms, (2004), URL: http://www-01.sil.org/linguistics/GlossaryOflinguisticTerms/ WhatIsDefiniteIdentifiability.htm (Zugriff vom 25.07.2015).

Loos (Hg.) 2004f = Loos, Eugene E. [u.a.] (Hg.), »What is definiteness «, in: Glossary Of Linguistic Terms, (2004), URL: http://www-01.sil.org/linguistics/GlossaryOflinguisticTerms/WhatIsDefiniteness.htm (Zugriff vom 25.07.2015).

Лукина/Чернецов (Нg.) 1935 = Лукина, Н.В. (Нg.), Вогульские сказки. Сборник фольклора народа манси (вогулов), St. Petersburg 1935.

Лукина (Нg.) 1990 = Лукина, Н. В. (Нg.), Мифыь, предания, сказки хантов и манси, Moskau 1990. Lyons 1999 = Lyons, Christopher, Definiteness, Cambridge 1999.

Lyons 1983 = Lyons, John, Semantik, 2, Cambridge 1983.

Lytkin/Majtinskaja (Hg.) 1966 = Lytkin, VasilijIl'ič/Majtinskaja, Klara E. (Hg.), Finno-ugorskie $i$ samodijskie jazyki [The finno-Ugric and Samoyedic languages] (Jazyki narodov SSSR, 3), Moskau 1966.

Majtinskaja 1970 = Majtinskaja, Klara E., " Possessivpronomina Sprachen«, in: Советское финно-угроведение 6 (1970), 271-275.

Manzelli 1990 = Manzelli, Gianguido, »Possessive adnominal modifiers«, in: Bechert, Johannes/ Bernini, Giuliano/Buridant, Claude (Hg.), Toward a Typology of European Languages (Empirical Approaches to Language Typology, 8), Berlin/New York 1990, 63-111.

McGregor (Hg.) 2009 = McGregor, William B. (Hg.), "Introduction«, in: McGregor William B. (Hg.), The Expression of Possession (The Expression of Cognitive Categories, ECC2), Berlin/ New York 2009, 1-12.

Merényi 1861 = Merényi, László, Eredeti Népmesék, Pest 1861, URL: http://mek.oszk. hu/08100/08132/08132.htm (Zugriff vom 29.09.2012).

Молданов 1997a = Молданов, Т.А., Олау ясуєм, Chanty-Mansijsk 1997.

Молданов 1997b = Молданов, Т.А, Кань кунш оляан 1, Chanty-Mansijsk 1997. 
Молданов 2001 = Молданов, Т.А., Кань кунш олуау 2, Tomsk 2001.

Molnár 2014 = Molnár, Krisztina, Substantivdetermination im Deutschen und im Ungarischen, Berlin 2014.

Moseley (Hg.) $2010=$ Moseley, Christopher (Hg.), Atlas of the World's Languages in Danger, Paris 2010, URL: http://www.unesco.org/culture/en/endangeredlanguages/atlas (Zugriff vom 09.03.2015).

Munkácsi/Kálmán (Hg.) 1986 = Munkácsi, Bernát/Kálmán, Béla (Hg.), Wogulisches Wörterbuch, Budapest 1986.

Murphy 1968 = Murphy, Lawrence Walter, Sosva Vogul Grammar, Indiana 1968.

Nagaya 2006 = Nagaya, Naonori, Topicality and Reference-Tracking in Tagalog, Tokio 2006, URL: http://www.ruf.rice.edu/ nn1/download/Nagaya2006Topicality_and_reference-tracking_ in_Tagalog.pdf (Zugriff vom 12.05.2014).

Nichols/Bickel 2013a = Nichols, Johanna/Bickel, Balthasar, $»$ Locus of Marking in Possessive Noun Phrases«, in: Dryer, Matthew S./Haspelmath, Martin (Hg.), The World Atlas of Language Structures Online, Leipzig 2013, URL: http://wals.info/chapter/24 (Zugriff vom 27.01.2014).

Nichols/Bickel 2013b = Nichols, Johanna/Bickel, Balthasar, »Possessive Classification «, in: Dryer, Matthew S./Haspelmath, Martin (Hg.), The World Atlas of Language Structures Online, Leipzig 2013, URL: http://wals.info/chapter/59 (Zugriff vom 27.01.2014).

Nikolaeva/Kovgan/Koškarëva 1993 = Nikolaeva, Irina A./Kovgan, Elena V./Koškarëva, Natalia, »Communicative roles in Ostyak syntax «, in: Finnisch-Ugrische Forschungen 51 (1993), 125-167.

Nikolaeva 1999a = Nikolaeva, Irina, Ostyak (Languages of the World/Materials, 305), München/ Newcastle 1999.

Nikolaeva 1999b = Nikolaeva, Irina, »The semantics of Northern Khanty evidentials", in: Journal de la Société Finno-Ougrienne 88 (1999), 131-159.

Nikolaeva 2001 = Nikolaeva, Irina, »The Hungarian external possessor in a European perspective«, in: Hasselblatt, Cornelius/Blokland, Rogier (Hg.), Finno-Ugrians and Indo- Europeans: Linguistic and Literary Contacts. Proceedings of the Symposium at the University of Groningen, November 22-24, 2002 (Studia Fenno-Ugrica Groningana, 2), Maastricht 2001, 272.

Nikolaeva 2002 = Nikolaeva, Irina, »Possession vs. nominal attribution in Uralic (with special attention to Ob-Ugric)«, in: Helimski, Eugen/Widmer, Anna (Hg.), Wuša wuša. Sei gegrüßt! Beiträge zur Finnougristik zu Ehren von Gert Sauer dargebracht zu seinem siebzigsten Geburtstag (Veröffentlichungen der Societas Uralo-Altaica, 57), Wiesbaden 2002, 239-250.

Nikolaeva 2003 = Nikolaeva, Irina, "Possessive affixes in the pragmatic structuring of the utterance: Evidence from Uralic«, in: Suihkonen, Pirkko/Comrie, Bernard (Hg.), International Symposium on Deictic Systems and Quantification in Languages Spoken in Europe and North and Central Asia. Udmurt State University, Izhevsk, Udmurt Republic, Russia, May 22-25, 2001, Collection of papers, Izhevsk/Leipzig 2003, 130.

Paczolay 1991 = Paczolay, Gyula, 750 magyar közmondás. 750 Ungarische Sprichwörter und Redewendungen, Veszprém 1991, URL: http://mek.oszk.hu/00200/00243/00243.htm (Zugriff vom 14.01.2015).

Payne/Barshi (Hg.) 1999 = Payne, Doris L./Barshi, Immanuel (Hg.), External possession (Typological Studies in Language, 39), Amsterdam/Philadelphia 1999.

Pierrehumbert 1980 = Pierrehumbert, Janet, »The Finnish Possessive Suffixes«, in: Language 56 (1980), 603-621.

Rédei 1965 = Rédei, Károly, Northern Ostyak Chresomathy (Uralic and Altaic Series, 47), Bloomington/The Hague 1965.

Rédei 1968 = Rédei, Károly, Nord-ostjakische Texte (Kazym-Dialekt) mit Skizze der Grammatik (Abhandlungen der Akademie der Wissenschaften in Göttingen. Philosophisch-Historische Klasse, Dritte Folge, 71), Göttingen 1968.

Reichert 1986 = Reichert, Christoph, Verteilung und Leistung der Personalaffixe im Ungarischen (Arbeitspapiere Köln, A.F. 52), Köln 1986. 
Riese 1987 = Riese, Timothy, »Zur Distribution der Allomorphe des Px3Sg im Konda-Wogulischen «, in: Rédei, Károly, Studien zur Phonologie und Morphonologie der uralischen Sprachen, Wien 1987, 204-210.

Riese 1990 = Riese, Timothy, »Nochmals zu Habeo im Wogulischen «, in: Pusztay, János, Gedenkschrift für Irén N Sebestyén (1890-1978) (Specimina Sibirica, 3), Pécs 1990, 175-180.

Riese 2001 = Riese, Timothy, Vogul (Languages of the world. Materials, 158), München 2001.

Röll 2011 = Röll, Daniela, »Reference tracking « in den ugrischen Sprachen (Hausarbeit zur Erlangung des akademischen Grades Magister Artium, unveröffentlichtes Manuskript), München 2011.

Ромбандеева 1996 = Ромбандеева, Евдокия Ивановна, Маньси мойтыт (Мансийские сказки), St. Petersburg 1996.

Rosch 1975 = Rosch, Eleanor, »Cognitive reference points«, in: Cognitive Psychology 7 (1975), 532-547.

Sammallahti 1998 = Sammallahti, Pekka, »Saamic«, in: Abondolo, Daniel, The Uralic Languages, London/New York 1998, 43-95.

Sanders/Gernsbacher 2004 = Sanders, Ted J. M./Gernsbacher, Morton Ann, »Accessibility in Text and Discourse Processing «, in: Discourse Processes 37/2 (2004), 79-89.

Schlachter 1960 = Schlachter, Wolfgang, Studien zum Possessivsuffix des Syrjänischen (FinnischUgrische Studien, 3), Berlin 1960.

Schmidt 1988 = Schmidt, Éva, »Az északi osztják birtokos névmásokról«, in: Domokos, Péter/ Pusztay, János (Hg.), Bereczki Emlékkönyv Bereczki Gábor 60 születésnapjára (Urálisztikai tanulmányok, 2), Budapest 1988, 319-324.

Schön 2014 = Schön, Zsófia, Postpositionale Konstruktionen in chantischen Dialekten (InauguralDissertation, unveröffentlichtes Manuskript), München 2014.

Schulze 1997 = Schulze, Brigitte, "Bedeutungsentlehnungen aus dem Russischen bei chantischen Postpositionen «, in: Hahmo, Sirkka-Liisa (Hg.), Vorträge des Symposiums aus Anlass des 30-jährigen Bestehens der Finnougristik an der Rijksuniversiteit Groninigen 21-23 November 1996, Maastricht 1997, 197-200.

Себурова (Нg.) 2009 = Себурова, Таисъя Стерановна (Hg.), Сказки божественной земли, Chanty-Mansijsk 2009.

Seiler 1973 = Seiler, Hansjakob, »Zum Problem der sprachlichen Possessivität«, in: Folia Linguistica 6 (1973), 231-250.

Seiler 1983a = Seiler, Hansjakob, Possession as an Operational Dimension of Language, Tübingen 1983.

Seiler 1983b = Seiler, Hansjakob, Possessivity, subject and object (Arbeiten des Kölner Universalien-Projekts, 43), Köln 1983.

Sherwood 2001 = Sherwood, Peter, »Definiteness in the Ugrian languages«, in: Seilenthal, Tõnu, Congressus Nonus Internationalis Fenno-Ugristarum, 7.-13.8.2000 Tartu, Pars IV, Tartu 2001, 185-187.

Siegl 2015 = Siegl, Florian, »The Structure of Noun Phrases with Referential PX.2P in Northern Samoyedic«, in: Tomsk Journal of Linguistics and Anthropology 7/1 (2015), 21-31, URL: http://ling.tspu.edu.ru/files/ling/PDF/articles/siegl_f._21_31_1_7_2015.pdf (Zugriff vom 24.05.2017).

Simonenko 2014 = Simonenko, Alexandra, »Microvariation in Finno-Ugric possessive markers Preprint «, in: Proceedings of NELS 43 (2014), 1-14, URL: http://people.linguistics.mcgill.ca/ alexandra. simonenko/Papers\&Handouts_files/SimonenkoNELS43.pdf (Zugriff vom 25.04.2015).

Sinor 1978 = Sinor, Denis, »The nature of possessive suffixes in Uralic and Altaic«, in: Jazayery, Mohammad Ali [u.a.] (Hg.), Linguistic and literary studies. In Honor of Archibald A. Hill, III. Historical and comparative linguistics (Trends in Linguistics. Studies and Monographs, 9), The Hague 1978, 257-266.

Sinor (Hg.) 1988 = Sinor, Denis (Hg.), The Uralic Languages. Description, History and Foreign Influences (Handbuch der Orientalistik, 1), Leiden/New York/Kopenhagen 1988. 
Skribnik 2001 = Skribnik, Jelena, »Pragmatic structuring in Northern Mansi«, in: Seilenthal, Tõnu, Congressus Nonus Internationalis Fenno-Ugristarum, 7.-13.8.2000 Tartu, Pars VI, Tartu 2001, 222-239.

Скрибник/Афанасева 2009 = Скрибник, Е. К./Афанасева, Клавдия, Практический курс мансийского языка 1-2 Ханты-Мансийск. In der Übersetzung als Kursmaterial für den Kurs Wogulisch I am Institut für Finnougristik/Uralistik, LMU München, Sommersemester 2009, München 2009.

Skribnik 2012 = Skribnik, Elena, Northern Mansi Miratives (Vortag gehalten auf der EuroBABEL Final Conference Leiden, NL), 2012, URL: http://babel.gwi.uni-muenchen.de/media/downloads/ grammar/NorthernMansi/Syntax/Northern_Mansi_Miratives.pdf (Zugriff vom 09.09.2013).

Соловар 1995 = Соловар, В. Н., Хантыйский язык. Учебник для 7-8 классов (казымсий диалект) (Югория: энцикл), Chanty-Mansijsk 1995.

Steinitz 1950 = Steinitz, Wolfgang, Ostjakische Grammatik und Chrestomathie mit Wörterverzeichnis, Leipzig 1950.

DEWOS/Steinitz 1966-1993 = Steinitz, Wolfgang, Dialektologisches und etymologisches Wörterbuch der ostjakischen Sprache. Lieferung 1-15 (Abhandlungen der deutschen Akademie der Wissenschaften zu Berlin. Klasse für Sprachen, Literatur und Kunst), Berlin 1966-1993.

Stolz $2008=$ Stolz, Thomas [u.a.], Split possession. An areal-linguistic study of the alienability correlation and related phenomena in the languages of Europe (Studies in Language Companion Series, 101), Amsterdam/Philadelphia 2008.

Suihkonen 2005 = Suihkonen, Pirkko, »On the Categories and Functions Developed from the Possessive and Deictic Suffixes in Udmurt «, in: Hasselblatt, Cornelius/Koponen, Eino/Widmer, Anna (Hg.), Lihkkun lehkos! Beiträge zur Finnougristik aus Anlaß des sechzigsten Geburtstages von Hans-Hermann Bartens (Veröffentlichungen der Societas Uralo-Altaica, 65), Wiesbaden 2005, 401-431.

Szabolcsi 1981 = Szabolcsi, Anna, »The Possessive Construction in Hungarian: A Configurational Categorie in a Non-Configurational Language«, in: Acta Linguistica Academiae Scientiarium Hungaricae 31 (1981), 261-289.

Szabolcsi 1987 = Szabolcsi, Anna, »Functional Categories in the Noun Phrase«, in: Approaches to Hungarian, 2. Theories and Analyses, Szeged 1987, 167-190.

Szabolcsi 1994 = Szabolcsi, Anna, »The Noun Phrase«, in: Kiefer, Ferenc/É. Kiss, Katalin (Hg.), The Syntactic Structure of Hungarian (Syntax and Semantics, 27), San Diego 1994, 179-274.

Szathmári 1988 = Szathmári, István, »La langue hongroise«, in: Sinor, Denis, The Uralic Languages. Description, History and Foreign Influences (Handbuch der Orientalistik), Leiden/New York/Kopenhagen 1988, 197-216.

Tauli 1966 = Tauli, Valter, Structural Tendencies in Uralic Languages (Indiana University Publications. Uralic and Altaic Series, 17), London 1966.

Taylor 1996 = Taylor, John R., Possessives in English: An Exploration in Cognitive Grammar, Oxford 1996.

Tolcsvai Nagy 2004 = Tolcsvai Nagy, Gábor, »Vázlat a magyar birtokos szerkezet jelentéstanáról «, in: Csepregi, Márta/Várady, Eszter (Hg.), Írások Szij Enikő 60 születésnapjára (Urálisztikai Tanulmányok, 14), Budapest 2004, 390-397.

Tribushinina 2008 = Tribushinina, Elena, Cognitive reference points: Semantics beyond the prototypes in adjectives of space and colour (Dissertation), Leiden 2008.

Ultan 1978 = Ultan, Russel, »Towards a typology of substantival possession ", in: Greenberg, Joseph H. (Hg.), Universals of human language, 4, Stanford CA 1978, 11-49.

Van Valin 1993 = Van Valin, Robert D., Role and reference grammar (Work Papers of the Summer Institute of Linguistics, University of North Dakota Session, 37), Washington 1993.

Vater 2001 = Vater, Heinz, Einführung in die Textlinguistik Struktur und Verstehen von Texten (UTB, 1660), München 2001.

Vater 2005 = Vater, Heinz, Referenz-Linguistik (UTB, 2685), München 2005. 
Vilkuna 2008 = Vilkuna, Maria, »\$ 891 Monikäyttöiset lausetyypit ja erikoislausetyypit«, in: Vilkuna, Maria, Iso suomen kielioppi verkkoversio, Helsinki 2008, URL: http://kaino.kotus.fi/visk/ sisallys.php? $\mathrm{p}=891$ (Zugriff vom 06.07.2015).

Willemse 2009 = Willemse, Peter [u.a.], »English Possessives as Reference-Point Constructions and their Function in the Discourse", in: McGregor William B. (Hg.), The Expression of Possession (The Expression of Cognitive Categories, ECC2), Berlin/New York 2009, 13-50.

Ziem 2008 = Ziem, Alexander, Kognitive Linguistik heute: ein kurzer Überblick über zentrale Positionen und Konzepte, 2008, URL: http://www.phil-fak.uni-duesseldorf.de/fileadmin/Redaktion/Institute/Germanistik/AbteilungI/Mitarbeiter/ziem/Ziem_KognitiveLinguistikHeute. pdf (Zugriff vom 17.09.2013). 
Die Beschreibung der Dimension der Possession - formale Ausdruckweisen, Interpretationen der Relationen und auch deren Einschränkungen - ist ein in der Linguistik seit dem Anfang des letzten Jahrhunderts gut dokumentiertes und erforschtes Themengebiet. In der Uralistik - der Sprachwissenschaft der uralischen Sprachen wie beispielsweise dem Finnischen und Ungarischen - lehnt sich die Beschreibung der Dimension Possession dabei stark an die Forschung zur Possession in den indo-europäischen Sprachen an. Beide Sprachfamilien unterscheiden sich jedoch typologisch, sodass auch die Strukturen zum Ausdruck von Possession mitunter stark voneinander abweichen. Dies führte unter anderem dazu, dass sich bei der Beschreibung des Possessivsuffixes, des Hauptbestandteils der Possessivkonstruktionen der uralischen Sprachen, eine Zweiteilung in possessive Funktionen und nicht-possessive Funktionen (d.h. solche Funktionen, die nicht mit dem Beschreibungsmodell anderer Sprachfamilien abgedeckt werden können) etabliert hat. Erst jüngere Abhandlungen beginnen, mit Berücksichtigung neuerer linguistischer Forschungsansätze, mit einer Re-Evaluation des Themas. So widmet sich dieses Buch einer Re-Analyse der Funktionen des Possessivsuffixes im ugrischen Sprachzweig der uralischen Sprachen (Ungarisch, Chantisch und Mansisch). Die Autorin verzichtet dabei auf die bisherige Zweiteilung possessiv und nicht-possessiv und beschreibt stattdessen das Possessivsuffix anhand seiner tatsächlichen Vorkommen im Text. Die Arbeit berücksichtigt anerkannte theoretische Grundlagen z.B. aus der kognitiven Linguistik, zieht neue Forschungsansätze wie die Informations-Strukturierung und die Referenz-Linguistik mit heran und ist in erster Linie korpus-basiert.

Gwen Eva Janda studierte Finnougristik/Uralistik, allgemeine und vergleichende Sprachwissenschaft sowie Slavistik an der Ludwig-Maximilians-Universität München und promovierte dort 2016 in der Graduiertenschule Sprache \& Literatur - Klasse für Sprache mit Stipendium der Bayerischen Eliteförderung. Interessens- und Forschungsgebiete sind bedrohte Sprachen Sibiriens und deren Dokumentation; vorwiegend das Erstellen von Korpora und die Datenanalyse, mit Schwerpunkt auf Morphosyntax und Pragmatik. Seit 2010 Mitarbeiterin in mehreren themenbezogenen DFG-Projekten.

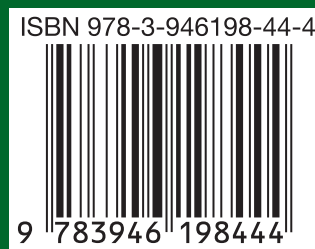

